\title{
KEISLER'S ORDER IS NOT SIMPLE (AND SIMPLE THEORIES MAY NOT BE EITHER)
}

\author{
M. MALLIARIS AND S. SHELAH
}

\begin{abstract}
Solving a decades-old problem we show that Keisler's 1967 order on theories has the maximum number of classes. In fact, it embeds $\mathcal{P}(\omega) /$ fin. The theories we build are simple unstable with no nontrivial forking, and reflect growth rates of sequences which may be thought of as densities of certain regular pairs, in the sense of Szemerédi's regularity lemma. The proof involves ideas from model theory, set theory, and finite combinatorics.
\end{abstract}

Keisler's order is a longstanding classification problem in model theory, introduced in 1967 [9] as a possible way of comparing the complexity of theories. Informally, say $T_{1} \unlhd T_{2}$ if the regular ultrapowers of models of $T_{1}$ are more likely to be saturated than those of $T_{2}$. Keisler's paper established that there was a minimum class, containing algebraically closed fields of fixed characteristic, and a maximum class, containing Peano arithmetic. By work of the second author in the seventies, see [33] Chapter VI, the union of the first two classes in Keisler's order gives an independent characterization of the stable theories, which are fundamental to modern model theory. Recently there has been much progress; for an account of work in the last decade, and some applications, see e.g. [21] or [18.

Among the questions raised by Keisler (see e.g. 10 p. 13) were how many classes the order had, whether it was linear, and what were syntactic characterizations of the minimum and maximum classes.

As of 1978 [33, the number was at least four, linearly ordered. Several years ago we discovered infinitely many classes, in fact an infinite descending chain 26], using certain hypergraphs first studied by Hrushovski [7]. Building on that construction, one can find conditional instances of nonlinearity (i.e. assuming a supercompact cardinal), as observed independently by Ulrich [37] and the authors 22. Recently, we found unconditional (ZFC) instances of nonlinearity [28. It would be consistent with these papers to conjecture that instances of nonlinearity were few, and that the number of equivalence classes was countable.

In the present paper we prove, in ZFC, that Keisler's order has the maximum number of classes (continuum many), by constructing a new family of simple unstable theories with no nontrivial forking which reflect growth rates of certain sequences of densities of finite graphs, and by developing new methods for building ultrafilters on Boolean algebras which carefully reflect these theories. (Both constructions seem quite flexible. Perhaps one reason some major structural conjectures about simple theories have remained stalled for decades is that simple unstable theories may have a much richer structure than previous examples suggest.)

Thanks. Partially supported by NSF CAREER award 1553653 and a Minerva Research Foundation membership at IAS (Malliaris), and European Research Council grant 338821 (Shelah), which with NSF 1362974 supported visits of the authors. This is paper 1167 in Shelah's list. 
The rough idea of our construction is as follows.

We first build pairs of infinite, finitely branching trees with an edge relation between nodes of corresponding height which thins out in an appropriate way as the height grows. In our main cas\&, whenever two nodes of height $k$ connect, their sets of immediate successors form a bipartite graph which, depending on the level, is either complete, or sparse and random (with a size and edge probability which is a function of the height). Associated to each height is a notion of "small" and "large" and the sparse graphs in question have the property that every small set of vertices has a common neighbor and no large set of vertices does. These structures, called parameters, can be thought of as encoding reduced graphs for the models of simple theories we then construct. The level function of a parameter is the set of heights at which we use sparse random (as opposed to complete) graphs; these are our choice of a way to track growth rates. To any such parameter we then associate a simple theory, essentially a kind of bipartite random graph filtered through unary predicates, which is simple unstable with no nontrivial forking. We prove that as the sequences of finite densities in the parameters vary sufficiently, as measured by the level functions, the associated theories have wildly different saturation behavior (a fundamentally infinitary phenomenon).

What happens on the ultrafilter side? For $\kappa$ regular and uncountable, we define a new chain condition to match the simple theories and which says, very roughly speaking, if we are given $\kappa$ positive elements of our Boolean algebra, then after moving to a subset $\mathcal{U}$ also of size $\kappa$, for any finite $n$ not in some ideal (of which more soon), any finite $u \subseteq \mathcal{U}$ which is "large" in the sense of level $n$ has a subset $v$ which is still "large" and whose elements are all compatible. The precise sense in which we choose our family of theories to be orthogonal to each other has to do with the fact that for any partition of our final set of parameters into $\mathcal{M}$ and $\mathcal{N}$, the ideal of subsets of $\omega$ generated by the subsets where the level functions of elements of $\mathcal{M}$ are 1 , does not contain ( $\bmod$ finite) the set where the level function of $\mathfrak{n}$ is 1 , for any $\mathfrak{n} \in \mathcal{N}$. (The idea is that if we preserve the chain condition using the ideal coming from $\mathcal{M}$, any future ultrafilter will omit at least one type in any theory from $\mathcal{N}$, since given any purported solution, the ultrafilter can concentrate too many of its conditions at points where they cannot all be satisfied.) Essentially this allows us to build by induction a (non-free!) Boolean algebra and a filter (eventually an ultrafilter) on it, adding formal solutions to problems coming from theories from $\mathcal{M}$ at suitable inductive stages, while preserving the chain condition using the ideal coming from $\mathcal{M}$ which ensures omission of a type for any theory with a parameter from $\mathcal{N}$. Previous model-theoretic constructions of ultrafilters were focused exclusively on free Boolean algebras; for details, see $\$ 8$

11 contains the statements of the main theorems.

These results suggest that not only do model theoretic dividing lines predict jumps in the complexity of theorems in finite combinatorics (as e.g. in stable regularity [19, or stable arithmetic regularity [36]), but also densities in the sense of finite combinatorics can control behavior of infinite models tightly enough that the resulting changes in complexity are detected by ultrafilters, so are candidates for model theoretic dividing lines.

The model theoretic, set theoretic, and combinatorial aspects of these constructions admit natural variations and raise interesting open questions, see 13 .

\footnotetext{
${ }^{1}$ This sketch describes theories with additional input from $\sqrt[6]{6}$ the frame in $\$ 2$ is more basic.
} 
Is this the end of a certain line of work on this problem? We think inversely: it tells us where to look.

We are grateful to very helpful questions and discussions after talks on a first version of this manuscript in summer and fall 2019 which improved the presentation and inspired us to prove some substantial new theorems in \$12. In particular, we thank M. Goldstern, I. Kaplan, M. Magidor, F. Parente, T. Scanlon, C. Terry, and M. Viale.

We are especially grateful to the anonymous referees for numerous, detailed, and extremely helpful comments on the manuscript. Thank you.

\section{Contents}

1. Notation and conventions 3

2. New theories 5

3. A dark woods 13

4. Models of $T_{\mathfrak{m}} \quad 16$

5. Ultrapowers of models of $T_{\mathfrak{m}}$

6. Sizes 22

7. Possibility patterns and ultrapowers 29

8. The chain condition 31

9. The c.c. and omitting types 38

10. The inductive construction 42

11. Main results and the big picture

12. Embedding $\mathcal{P}(\omega) /$ fin $\quad 60$

13. Further discussion and open questions 72

References

\section{Notation AND CONVEntions}

Convention 1.1. Unless otherwise stated, all graphs are simple graphs: no loops and no multiple edges.

Convention 1.2. We will often write bipartite graphs as triples $(V, W, E)$, where $V, W$ are the sets of vertices and the $E \subseteq V \times W$ is the edges. We will call a bipartite graph complete if $E=V \times W$, so in this case $E$ is asymmetric.

In this paper we will have both finite and infinite (possibly uncountable) random graphs; the infinite ones are random in the sense of model theory, which should not cause confusion. The next two definitions, ordinary 1.3 and bipartite 1.4, explain what this means.

Convention 1.3 (The model-theoretic random graph). "The theory of the random graph" means the set of first-order axioms in the language with a binary relation symbol E, and equality, which say that $E$ is symmetric irreflexive, that there are infinitely many elements, and for any two finite disjoint sets $v, w$, there is a vertex $a$ such that $E(a, b)$ for all $b \in v$ and $\neg E(a, c)$ for all $c \in w$.

Convention 1.4 (Infinite bipartite random graphs). If $(A, B, E)$ is a bipartite graph and $A, B$ are infinite, we may call it a bipartite random graph to mean that the following two conditions hold: for any two finite disjoint $u, v \subseteq B$, there is 
$a \in A$ such that $\bigwedge_{b \in u} E(a, b) \wedge \bigwedge_{b \in v} \neg E(a, b)$, and conversely, for any two finite disjoint $u, v \subseteq A$, there is $b \in B$ such that $\bigwedge_{a \in u} E(a, b) \wedge \bigwedge_{a \in v} \neg E(a, b)$.

Convention 1.5 (Trees). Recall that a tree is a partially ordered set such that the set of predecessors of any given node is well ordered, so in particular linearly ordered. In this paper, the partial order will always be given by initial segment, denoted $\unlhd$. In this paper, all trees will be finitely branching, and all nodes of all trees will have finite height, so any tree will be either of finite or countable height.

Remark 1.6. The symbol $\unlhd$ is used in this paper to denote two unrelated kinds of partial orders: to denote the partial order on elements of a given tree and to denote Keisler's ordering on theories. Since these two contexts never overlap, this should not cause any confusion.

Definition 1.7. As usual, we denote by $\mathfrak{B}_{\alpha, \mu, \aleph_{0}}^{0}$ the free Boolean algebra generated by $\alpha$ independent partitions each of size $\mu$, and $\mathfrak{B}_{\alpha, \mu, \aleph_{0}}^{1}$ is its completion.

The last subscript, $\aleph_{0}$, in 1.7 refers to the fact that any intersection of $<\aleph_{0}$ elements from distinct partitions is nonempty. Often it is understood and so not written. When it takes values other than $\aleph_{0}$, we usually refer to it as $\theta$ - this was used, for instance, in 28 . On the existence of $\mathfrak{B}_{2^{\lambda}, \mu}^{0}$, i.e. $\mathfrak{B}_{2^{\lambda}, \mu, \aleph_{0}}^{0}$, when $\lambda \geq \mu$ see e.g. Fichtenholz-Kantorovich, or Hausdorff, or [33] Appendix Theorem 1.5. When $\theta>\aleph_{0}$, the existence theorem requires $\lambda=\lambda^{<\theta} \geq \mu$. In this paper, to find $D_{0}, \mathcal{D}_{*}, \mathbf{j}$ as in 7.1 below, we use the completion.

Definition 1.8. Let

$$
\mathrm{FI}_{\mu, \theta}(\alpha)=\{h: h \text { is a function, } \operatorname{dom}(h) \subseteq \alpha, \operatorname{range}(h) \subseteq \mu,|\operatorname{dom}(h)|<\theta \text {. }\}
$$

When $\theta=\aleph_{0}$, as is often our case in this paper, we usually omit it.

This notation recalls e.g. 33. Definition 3.6 p. 358; for a more detailed explanation, see [28] §1. In our context, the simple idea is as follows. We are dealing with, say, a Boolean algebra $\mathfrak{B}_{\alpha, \mu, \aleph_{0}}^{1}$ which is the completion of a Boolean algebra generated freely by $\alpha$ many independent partitions (=maximal antichains) each of size $\mu$. Notice that if we enumerate the generators as $\left\langle\mathbf{x}_{\beta, \epsilon}: \beta<\alpha, \epsilon<\mu\right\rangle$ then the intersection of finitely many [or in general, fewer than $\theta$ ] elements $\mathbf{x}_{\beta_{i}, \epsilon_{i}}$ $\left(i<i_{*}<\omega\right)$ will be nonzero if and only if we haven't chosen two distinct elements from the same antichain, that is, if $\left\{\left(\beta_{i}, \epsilon_{i}\right): i<i_{*}\right\}$ is a function. Elements arising from such intersections will be dense in the completion and merit compact notation. So we shall specify elements arising as such intersections by functions $f$ such that $\operatorname{dom}(f) \subseteq \alpha,|\operatorname{dom}(f)|<\aleph_{0}$, and range $(\alpha) \subseteq \mu$, where again, we think of such a function as selecting an element from each of a small number of antichains which are then intersected to form the nonzero element " $\mathbf{x}_{f}$ ". Indeed, we could make " $\mathbf{x}_{\beta, \epsilon}$ " a special case of this notation by specifying that when the domain of the function has size 1 we may drop the parentheses in $\mathbf{x}_{\{(\beta, \epsilon)\}}$. Thus [1.8, and thus:

Convention 1.9. For $g \in \mathrm{FI}_{\mu, \theta}(\alpha)$, let $\mathbf{x}_{g}$ denote the corresponding nonzero element of $\mathfrak{B}$.

As the generators are dense in the completion, we have:

Remark 1.10. Let $\mathfrak{B}=\mathfrak{B}_{\alpha, \mu, \aleph_{0}}^{1}$. Then, in our notation, the elements of the form $\mathbf{x}_{f}$ for $f \in \mathrm{FI}_{\aleph_{0}}(\alpha)$ are dense in $\mathfrak{B}$. 
Fact $1.11(\Delta$-system lemma, see e.g. Kunen [12] III.6.15). Let $\nu$ and $\kappa$ be regular cardinals such that $\aleph_{0} \leq \nu<\kappa$. Assume that $(\forall \alpha<\kappa)\left(\alpha^{<\nu}<\kappa\right)$. Let $\mathcal{A}$ be a family of sets with $|\mathcal{A}|=\kappa$, such that $|A|<\nu$ for all $A \in \mathcal{A}$. Then there is a $\mathcal{B} \subseteq \mathcal{A}$ of size $\kappa$ such that $\mathcal{B}$ forms a $\Delta$-system.

Note that the family of sets need not be subsets of $\kappa$; we place no restriction on their provenance, only restrictions on size of the family and size of the sets. We will mostly use the case $\nu=\aleph_{0}$ :

Corollary 1.12. If $\kappa$ is an uncountable regular cardinal and $\mathcal{A}$ is a family of $\kappa$ sets, all of them finite, there is $\mathcal{B} \subseteq \mathcal{A}$ of size $\kappa$ which forms a $\Delta$-system.

A central definition in this paper will be Keisler's order. For more on the order, see [9], or for example the extended introduction to [25]. Some key points:

Definition 1.13 (Keisler's order, 99). Let $T_{1}, T_{2}$ be complete countable first-order theories. We say $T_{1} \unlhd T_{2}$ if for every infinite $\lambda$, every regular ultrafilter $\mathcal{D}$ on $\lambda$, every model $M_{1} \models T_{1}$, and every model $M_{2} \models T_{2}$, if $\left(M_{2}\right)^{\lambda} / \mathcal{D}$ is $\lambda^{+}$-saturated, then $\left(M_{1}\right)^{\lambda} / \mathcal{D}$ is $\lambda^{+}$-saturated.

Recall that the ultrafilter $\mathcal{D}$ on $\lambda$ is regular if there exists a regularizing family, meaning $X=\left\{X_{\alpha}: \alpha<\lambda\right\} \subseteq \mathcal{D}$ such that the intersection of any infinitely many elements of $X$ is empty. By a lemma of Keisler [9, 2.1], if $\mathcal{D}$ is regular, then the choice of $M_{1}, M_{2}$ in 1.13 does not matter, up to elementary equivalence. For more on regular ultrafilters, see [2] $\S 4.3$ and $\S 6.1$.

Recall that a regular ultrafilter $\mathcal{D}$ on $\lambda$ is $\lambda^{+}$-good if every $f:[\lambda]^{<\aleph_{0}} \rightarrow \mathcal{D}$ which is monotonic has a multiplicative refinement, that is, if $u \subseteq v$ implies $f(u) \supseteq f(v)$ for all $u, v \in[\lambda]^{<\aleph_{0}}$, then there exists $g:[\lambda]^{<\aleph_{0}} \rightarrow \mathcal{D}$ such that $g(u) \subseteq f(u)$ for all $u \in[\lambda]^{<\aleph_{0}}$ and $g(u) \cap g(v)=g(u \cup v)$ for all $u, v \in[\lambda]^{<\aleph_{0}}$.

Keisler [9] proved that good ultrafilters characterize the maximum class in Keisler's order: if $\mathcal{D}$ is a regular ultrafilter on $\lambda$, then $\mathcal{D}$ is $\lambda^{+}$-good if and only if $M^{\lambda} / \mathcal{D}$ is $\lambda^{+}$-saturated for some, equivalently every, model of every complete countable theory $T$. By extension,

Convention 1.14. If $\mathcal{D}$ is a regular ultrafilter on $\lambda$ and $\kappa \leq \lambda$ and $T$ is a complete countable theory, we may say

$$
\mathcal{D} \text { is }\left(\kappa^{+}, T\right) \text {-good }
$$

if for some, equivalently every, $M \models T$ we have that $M^{\lambda} / \mathcal{D}$ is $\kappa^{+}$-saturated. When $\kappa=\lambda$, we may just say " $\mathcal{D}$ is good for $T$." Note that the negation, " $\mathcal{D}$ is not $\left(\kappa^{+}, T\right)$-good" means that for some, equivalently every, $M \models T$, the ultrapower $M^{\lambda} / \mathcal{D}$ is not $\kappa^{+}$-saturated.

\section{NeW THEORIES}

This section defines a new family of simple theories.

Here is a brief overview. First we define parameters, and from these, we define our new theories. A parameter is a kind of template. It is essentially a pair of finitely branching trees, of countable height, along with data about whether nodes in the left tree do or do not connect to nodes of the corresponding height (=level) in the right tree. There are a series of coherence conditions which say things like: 
the branching gradually increases; in order for two nodes to connect, all of their initial segments must have been connected, but this isn't sufficient: two nodes at level $n$ which connect may have many immediate successors which don't connect, but at least a few must. There is also a level function, which is 0 or 1 at each level, and 1 infinitely often.

In our main case in the present paper, the connection patterns in our trees will have a particularly elegant form. We will first specify that all nodes at level $n$, in both trees, have the same number of successors. We shall then choose in advance a distinguished, growing sequence of bipartite (fairly random) graphs with the correct number of vertices, and recall we have the given level function. At each level $n$, if the level function is 1 (an "active level"), whenever two nodes are connected, we let the pattern of connections among their immediate successors be given by the distinguished bipartite graph for level $n+1$. If the level function is 0 (a "lazy level," the idea being there are no additional constraints introduced at this level), we let the pattern of connections among their immediate successors be given by a complete bipartite graph. Either way, if two nodes are not connected, there are no connections between their immediate successors. In such cases, we can reasonably say that the tree is built over a sequence of graphs. However, the construction is more flexible, and doesn't require patterns of connections to be essentially invariants of the level; so in this section and the next, we work out the construction at a somewhat greater level of generality.

After defining parameters, we will continue these remarks (before 2.14) to motivate the theories built from given parameters. Recall convention 1.5 on trees.

Notation 2.1 (Notation for trees).

(1) In this section, a tree will always denote a subset of ${ }^{\omega>} \omega$, closed under initial segments, and partially ordered by initial segment, denoted $\unlhd$.

(2) For $\mathcal{T}_{i}$ a tree and $k<\omega$, let $\mathcal{T}_{i, k}$ denote the $k$ th level of $\mathcal{T}_{i}$, i.e

$$
\mathcal{T}_{i, k}=\mathcal{T}_{i} \cap{ }^{k} \omega .
$$

That is, that any $\eta \in \mathcal{T}_{i, k}$ has length $k$ and $\mathbb{W}^{2}$ is a function from $\{0, \ldots, k-1\}$ to $\omega$ (so if $k=0$, then $\operatorname{dom}(\eta)=\emptyset$ ). We may write $\eta(t)$ for the value of $\eta$ at $t \in \operatorname{dom}(\eta)$.

(3) Let $\mathcal{T}_{i, \leq k}$ denote $\bigcup_{\ell \leq k} \mathcal{T}_{i, \ell}$.

(4) For $\mathcal{T}_{i}$ a tree and $\eta \in \mathcal{T}_{i, k}$, denote the immediate successors of $\eta$ in $\mathcal{T}_{i}$ by

$$
\operatorname{ims}_{\mathcal{T}_{i}}(\eta)=\left\{\eta^{\prime} \in \mathcal{T}_{i, k+1}: \eta \unlhd \eta^{\prime}\right\} .
$$

(5) For $\mathcal{T}_{i}$ a tree, denote the leaves of $\mathcal{T}_{i}$ by

$$
\lim \left(\mathcal{T}_{i}\right)=\left\{\eta \in{ }^{\omega} \omega: \omega\left\lceil k \in \mathcal{T}_{i, k} \text { for all } k<\omega\right\} .\right.
$$

Definition 2.2. Call $\xi: \omega \rightarrow\{0,1\}$ a level function if $\{i<\omega: \xi(i)=1\}$ is infinite, and (for convenience) $\xi(0)=1$.

The idea of a level function, 2.2. will be that level $i$ of the tree is active if $\xi(i)=1$, and not if $\xi(i)=0$ (the "lazy levels"), explained presently.

The first main ingredient is that of a parameter (basic parameter 2.3, parameter 2.6) which will give us the blueprint on which a theory can be based.

\footnotetext{
${ }^{2}$ Note that under this setup the "Oth level" is a singleton, i.e $\mathcal{T}_{1,0}=\mathcal{T}_{2,0}=\{\emptyset\}$.
} 
Definition 2.3. A basic parameter $\mathfrak{m}$ consists of a pair of trees $\mathcal{T}_{1}, \mathcal{T}_{2}$, a sequence of binary relations $\mathcal{R}_{k}$ for $k<\omega$, and a level function $\xi$, all satisfying the following.

(1) $\mathcal{T}_{1}$ and $\mathcal{T}_{2}$ are subtrees of ${ }^{\omega>} \omega$ with finite splitting and no maximal node.

(2) For $k<\omega, \mathcal{R}_{k} \subseteq \mathcal{T}_{1, k} \times \mathcal{T}_{2, k}$, and for $k=0$ we have equality.

(3) If $\left(\eta_{1}, \eta_{2}\right) \in \mathcal{R}_{k+1}$ then $\left(\eta_{1}\left\lceil k, \eta_{2}\lceil k) \in \mathcal{R}_{k}\right.\right.$.

(4) If $\left(\eta_{1}, \eta_{2}\right) \in \mathcal{R}_{k}, \eta_{2}^{\prime} \in \operatorname{ims}_{\mathcal{T}_{2}}\left(\eta_{2}\right)$, then for at least two distinct $\eta_{1}^{\prime}, \eta_{1}^{\prime \prime} \in$ $\operatorname{ims} \mathcal{T}_{1}\left(\eta_{1}\right)$ we have $\left(\eta_{1}^{\prime}, \eta_{2}^{\prime}\right) \in \mathcal{R}_{k+1}$ and $\left(\eta_{1}^{\prime \prime}, \eta_{2}^{\prime}\right) \in \mathcal{R}_{k+1}$, and the parallel for the trees reversed. [Informally, if two elements at one level are connected, then every immediate successor of one of them is connected to at least two immediate successors of the other.]

(5) If $\xi(k)=0$, then $\left(\mathcal{T}_{1, k+1}, \mathcal{T}_{2, k+1}, \mathcal{R}_{k+1}\right)$ adds no new constraints meaning: if $\left(\eta_{1}, \eta_{2}\right) \in \mathcal{T}_{1, k+1} \times \mathcal{T}_{2, k+1}$ and $\left(\eta_{1}\left\lceil k, \eta_{2}\lceil k) \in \mathcal{R}_{k}\right.\right.$ then $\left(\eta_{1}, \eta_{2}\right) \in \mathcal{R}_{k+1}$.

(6) Let $\mathcal{R}=\bigcup_{k<\omega} \mathcal{R}_{k}$.

Corollary 2.4. It follows from Definition 2.3(4) that we may add:

(7) Fullness: For every $\eta \in \lim \left(\mathcal{T}_{1}\right)$, there are continuum many $\rho \in \lim \left(\mathcal{T}_{2}\right)$ such that $\left(\eta\left\lceil k, \rho\lceil k) \in \mathcal{R}_{k}\right.\right.$ for all $k<\omega$.

Likewise, for every $\rho \in \lim \left(\mathcal{T}_{2}\right)$, there are continuum many $\eta \in \lim \left(\mathcal{T}_{1}\right)$ such that $\left(\eta\left\lceil k, \rho\lceil k) \in \mathcal{R}_{k}\right.\right.$ for all $k<\omega$.

(Of course, we could have just stated this as a separate axiom.)

Remark 2.5. On the level functions. Conditions 2.3(5)-(6) tell us essentially that if $\xi(i)=0, \mathcal{R}_{i+1}$ is set by (2.3) (5) and contributes no new constraints: if two elements connect in $\mathcal{R}_{i}$, then $\mathcal{R}_{i+1}$ is a complete bipartite graph on their immediate successors, whereas if two elements don't connect in $\mathcal{R}_{i}, \mathcal{R}_{i+1}$ is an empty graph on their successors. We call $i$ a "lazy level" (we chose to say this about $i$, although we could have said this about $i+1)$. In contrast if $\xi(i)=1$, we will have a lot of freedom in choosing $\mathcal{R}_{i+1}$, subject to 2.3(6) and 2.6. The usefulness of this feature, the level function, will be more apparent starting in $\$ 6$ when we pattern the $\mathcal{R}_{i}$ 's on tailor-made sequences of bipartite random graphs, and start comparing theories whose level functions are in some natural sense independent.

Since we will be interested in varying the edge families $\mathcal{R}_{k}$, the following conditions will ensure there are a minimum of edges and edge coherence to define a model completion. In the rest of this paper, we will always assume them to be true. We could have included them in 2.3 .

Definition 2.6. We say the basic parameter $\mathfrak{m}$ is a parameter when, in addition 3

(1) Left extension: if $k<\omega, \nu \in \mathcal{T}_{2, k}, u \subseteq \mathcal{T}_{1, k+1},|u| \leq k$ satisfy

$$
(\forall \eta \in u)\left[\left(\eta\lceil k, \nu) \in \mathcal{R}_{k}\right]\right.
$$

then there are $\geq k+1$ elements $\rho \in \operatorname{ims} \mathcal{T}_{2}(\nu)$ such that

$$
(\forall \eta \in u)\left[(\eta, \rho) \in \mathcal{R}_{k+1}\right] .
$$

(2) Right extension: if $k<\omega, \nu \in \mathcal{T}_{1, k}, u \subseteq \mathcal{T}_{2, k+1},|u| \leq k$ satisfy

$$
(\forall \rho \in u)\left[\left(\nu, \rho\lceil k) \in \mathcal{R}_{k}\right]\right.
$$

then there are $\geq k+1$ elements $\eta \in \operatorname{ims}_{\mathcal{T}_{1}}(\nu)$ such that

$$
(\forall \rho \in u)\left[(\eta, \rho) \in \mathcal{R}_{k+1}\right] .
$$

\footnotetext{
${ }^{3}$ We repeat the conditions for both sides since $\mathcal{R}_{k}$ is not required to be symmetric.
} 
Remark 2.7. Together, 2.3 and the extension axioms of 2.6 imply that the branching of each $\mathcal{T}_{\ell}$ at height $k$ is at least $k+1$.

Remark 2.8. Note that extension does not require the elements in the set $u$ to have an immediate common predecessor.

As the results of this paper indicate it may be interesting to further investigate theories in this region, we include two comments on alternative definitions.

Discussion 2.9. We could have weakened left and right extension by asking, e.g.: for every $k_{1}<\omega$ there is $k_{2}>k_{1}$ such that if $k_{3} \geq k_{2}, \nu \in \mathcal{T}_{3-\ell}, k_{3}, u \subseteq \mathcal{T}_{\ell, k_{3}+1}$, $|u| \leq k_{1}$ satisfy, etc. With this we would gain a little in some places, e.g. 2.13, and lose a little in others, e.g. 2.19, The clean formulation in 2.6 is sufficient for our purposes here. Informally, rather than working with a fixed branching and letting the number of connections be arbitrarily slow-growing, we encode $f(k) \geq k+1$ in our extension axioms and in the construction allow branching to be arbitrarily large.

Discussion 2.10. Another variation we do not use here would be to say $\mathfrak{m}$ is very nice when we may add a non-connection clause to the extension axioms, e.g. if $k<\omega, \nu \in \mathcal{T}_{2, k}, u, v \subseteq \mathcal{T}_{1, k+1}$, are disjoint, $|u \cup v| \leq k$ satisfy

$$
(\forall \eta \in u)\left[\left(\eta\lceil k, \nu) \in \mathcal{R}_{k}\right]\right.
$$

then there are $\geq k+1$ elements $\rho \in \operatorname{ims}_{\mathcal{T}_{2}}(\nu)$ such that $(\forall \eta \in u)\left[(\eta, \rho) \in \mathcal{R}_{k+1}\right]$ and $(\forall \eta \in v)\left[(\eta, \rho) \notin \mathcal{R}_{k+1}\right]$ - and similarly for $\mathcal{T}_{1}, \mathcal{T}_{2}$ reversed $\mathbb{4}^{[4}$

Returning to the main line of the construction, an important feature of this setup is its potential for symmetry, which will help in our proofs.

Definition 2.11. For any parameter $\mathfrak{m}_{1}$, the dual $\mathfrak{m}_{2}=\operatorname{dual}\left(\mathfrak{m}_{1}\right)$ is defined by:

(1) $\left(\mathcal{T}_{2}^{\mathfrak{m}_{2}}, \mathcal{T}_{1}^{\mathfrak{m}_{2}}\right)=\left(\mathcal{T}_{1}^{\mathfrak{m}_{1}}, \mathcal{T}_{2}^{\mathfrak{m}_{1}}\right)$.

(2) $\mathcal{R}_{n}^{\mathfrak{m}_{2}}=\left\{\left(\eta_{2}, \eta_{1}\right):\left(\eta_{1}, \eta_{2}\right) \in \mathcal{R}_{n}^{\mathfrak{m}_{1}}\right\}$

Observation 2.12. If $\mathfrak{m}$ is a basic parameter, so is $\operatorname{dual}(\mathfrak{m})$, and $\operatorname{dual}(\operatorname{dual}(\mathfrak{m}))=$ $\mathfrak{m}$, and if $\mathfrak{m}$ is a parameter, then so is $\operatorname{dual}(\mathfrak{m})$.

It is worth noting that this definition extends the "new simple theory" from [28, used there to produce an example of incomparability in ZFC. That said, the present version is substantially more general and more flexible, both in its set-up and in its incorporation of symmetry, as the next sections will show. [The reader unfamiliar with [28, can safely skip Observation 2.13.]

Observation 2.13. For every $f: \omega \rightarrow \omega \backslash\{0,1,2\}$ which goes to infinity, $T_{f}$ from 28 is equal, up to renaming, to $T_{\mathfrak{m}}$ for some basic parameter $\mathfrak{m}$. If in addition $f(k) \geq k+1$, then in addition $\mathfrak{m}$ is a parameter.

Proof. Using the notation of 28] Definition 2.4, let's check definitions 2.3 and 2.6 Let $\mathcal{T}_{2, n}=\prod_{\ell<n} f(\ell)$ and let $\mathcal{T}_{2}=\bigcup_{n} \mathcal{T}_{2, n}$.

In order to define $\mathcal{T}_{1}$, recall that in [28] 2.4, there was a natural tree structure on the left-hand side given as follows. We called $s \subseteq \mathcal{T}_{1, \leq k}$ " $k$-maximal" if (a) it is a subtree, thus downward closed (closed under initial segment), and (b) it does not contain all immediate successors of any given node. The point is that an element of the left-hand side in a model of $T_{f}$ determined some such $s$ (by its connections

\footnotetext{
${ }^{4}$ One drawback is that this isn't satisfied by the theories of 28 .
} 
on the right) and that $\subseteq$ gives a natural partial ordering on the set of all $s$ that are $k$-maximal for some finite $k$, forming an infinite, finitely branching tree. So, we choose $\mathcal{T}_{1} \subseteq{ }^{\omega>} \omega$ to be equivalent to this tree (up to renaming) and choose $\mathcal{R}$ so that $\mathcal{R}_{k}$ holds between $\eta \in \mathcal{T}_{1, k}$ and $\rho \in \mathcal{T}_{2, k}$ if and only if $\eta$ was (before renaming) the subtree $s$ and $s$ contains $\rho$. Let $\xi$ be the sequence constantly equal to 1 . This completes the specification of $\mathfrak{m}$, so let us check 2.3. Clearly (1), (2), (3), (4), (6) hold. (5) is trivially satisfied as $\xi$ is constantly 1 . Likewise, it is straightforward to check that as long as $f(k) \geq k+1$, the fullness and extension conditions of 2.6] follow easily from the use of $k$-maximal $s$ 's. Thus, $\mathfrak{m}$ is a parameter.

Next we use our template $\mathfrak{m}$ to produce a universal theory, and its model completion. Note that this theory is in a different signature, and a priori has no access to the trees and edges mentioned in $\mathfrak{m}$.

First we informally describe this universal theory. The signature consists of unary predicates, explained next, plus a binary relation $R$. The unary predicates are indexed by the nodes in the trees $\left(Q_{\eta}\right.$ for the left, $P_{\nu}$ for the right, along with " $\mathcal{Q}$ " for $Q_{\langle\rangle}$and " $\mathcal{P}$ " for $\left.P_{\langle\rangle}\right)$and these predicates in some sense "hard-code" the structure of the trees: $\mathcal{Q}$ and $\mathcal{P}$ partition the domain, the $Q_{\eta}$ 's are all subsets of $\mathcal{Q}$, the $P_{\nu}$ 's are all subsets of $\mathcal{P}$, if $\eta \unlhd \eta^{\prime}$ then $Q_{\eta} \supseteq Q_{\eta^{\prime}}$, if $\nu_{1}, \nu_{2}$ are immediate successors of $\eta$ then $Q_{\nu_{1}} \cap Q_{\nu_{2}}=\emptyset$ (indeed, the predicates corresponding to the immediate successors of $\eta$ partition $Q_{\eta}$ ), and the parallel for the $P$ 's. This can all be said with universal axioms. Finally, we need to address $R$, which is a binary relation which may hold between elements of $\mathcal{Q}$ and elements of $\mathcal{P}$. We add universal axioms saying essentially that if $\eta$ and $\nu$ were nodes of the same level in the left and right trees respectively which were not connected in the template, then $R$ cannot hold between any element of $Q_{\eta}$ and $P_{\nu}$. (Now it should be clearer why we remarked that the "lazy level adds no new constraints.")

It will be convenient to define and work with the finite approximations $T_{\mathfrak{m}, k}^{0}$ where we only have unary predicates for nodes up to level $k$ of the left and right trees. So $T_{\mathfrak{m}}^{0}$, the theory we've just sketched, will be a universal theory in an infinite language, defined as the union of $T_{\mathfrak{m}, k}^{0}$ for all finite $k$.

$\$ 3$ contains a more precise discussion of such theories and the model completions $T_{\mathfrak{m}}$, whose existence we will of course have to justify in the rest of this section. Very informally for now [this remark is made more precise in 93 , the model completion may be thought of as a "bipartite random graph filtered through trees," in the sense that if we are in a sufficiently saturated 5 model of $T_{\mathfrak{m}}$, whenever we take a type-definable set on the left corresponding to a leaf in the left template tree, and likewise a type-definable set on the right corresponding to a leaf in the right template tree, then if these two leaves were "connected all the way up" in the template, the restriction of $R$ to these two sets in our model will look like an infinite bipartite random graph, in the sense of model theory.

Remark 2.14. When the context is clear, below, we will write $\mathfrak{m}=\left(\mathcal{T}_{1}, \mathcal{T}_{2}, \mathcal{R}\right)$ instead of $\left(\mathcal{T}_{\mathfrak{m}, 1}, \mathcal{T}_{\mathfrak{m}, 2}, \mathcal{R}_{\mathfrak{m}}\right)$.

In the next definition, we informally think of $\mathcal{Q}$ as being on the left and $\mathcal{P}$ as being on the right.

\footnotetext{
${ }^{5}$ Some saturation is assumed just so that both type-definable sets are infinite.
} 
Definition 2.15. Given a parameter $\mathfrak{m}$ and $k<\omega$, define $T_{\mathfrak{m}, k}^{0}$, a universal first order theory, as follows. Let $\tau_{k}=\tau_{\mathfrak{m}, k}$ denot 6

$$
\left\{\mathcal{Q}, \mathcal{P}, Q_{\eta}, P_{\rho}: \eta \in \mathcal{T}_{1, \leq k}, \rho \in \mathcal{T}_{2, \leq k}\right\} \cup\{R\} .
$$

Then $T_{\mathfrak{m}, k}^{0}$ is the universal theory in $\mathcal{L}\left(\tau_{\mathfrak{m}, k}\right)$ such that a $\tau_{k}$-model $M$ is a model of $T_{\mathfrak{m}, k}^{0}$ if and only if:

(1) $\mathcal{Q}^{M}, \mathcal{P}^{M}$ is a partition of $\operatorname{dom}(M)$. We identify $\mathcal{Q}$ and $Q_{\langle\rangle}, \mathcal{P}$ and $P_{\langle\rangle}$.

(2) $\left\langle Q_{\eta}^{M}: \eta \in \mathcal{T}_{1, n}\right\rangle$ is a partition of $\mathcal{Q}^{M}$ for each $n \leq k$ and this partition satisfies

$$
\eta \unlhd \nu \in \mathcal{T}_{1, \leq k} \text { implies } Q_{\eta}^{M} \supseteq Q_{\nu}^{M} .
$$

(3) $\left\langle P_{\rho}^{M}: \rho \in \mathcal{T}_{2, n}\right\rangle$ is a partition of $\mathcal{P}^{M}$ for each $n \leq k$ and this partition satisfies

$$
\rho \unlhd \nu \in \mathcal{T}_{2, \leq k} \text { implies } P_{\rho}^{M} \supseteq P_{\nu}^{M} .
$$

(4) $R^{M} \subseteq\left\{(b, a): b \in Q^{M}, a \in P^{M}\right.$ and for every $n \leq k$, there are $\eta_{1} \in \mathcal{T}_{1, n}$, $\eta_{2} \in \mathcal{T}_{2, n}$ such that $\left(\eta_{1}, \eta_{2}\right) \in \mathcal{R}_{n}$ and $\left.(b, a) \in Q_{\eta_{1}}^{M} \times P_{\eta_{2}}^{M}\right\}$.

Informally, condition 2.15(4) says there can only be $R$-edges in $M$ between elements which belong to "leaves" all of whose initial segments of the same height were connected in the template $\mathcal{R}$.

Observation 2.16. $T_{\mathfrak{m}, k}^{0} \subseteq T_{\mathfrak{m}, k+1}^{0}$.

Definition 2.17. Given a parameter $\mathfrak{m}$ we define $T_{\mathfrak{m}}^{0}$, a universal first order theory, as follows. The vocabulary is $\tau=\tau_{\mathfrak{m}}=\left\{\mathcal{Q}, \mathcal{P}, Q_{\eta}, P_{\nu}, R: \eta \in \mathcal{T}_{1}, \nu \in \mathcal{T}_{2}\right\}$ where $\mathcal{Q}, \mathcal{P}, Q_{\eta}, P_{\nu}$ are unary predicates and $R$ is a binary predicate, and

$$
T_{\mathfrak{m}}^{0}=\bigcup\left\{T_{\mathfrak{m}, k}^{0}: k<\omega\right\} .
$$

Claim 2.18. For each $k<\omega$, the model completion $T_{\mathfrak{m}, k}$ of $T_{\mathfrak{m}, k}^{0}$ exists.

Proof. $T_{\mathfrak{m}, k}^{0}$ is a universal theory in a finite relational language, and the class of its models has JEP and AP. Suppose we are given any two $M_{1}, M_{2} \models T_{\mathfrak{m}, k}^{0}$. For JEP, we also assume $M_{1} \cap M_{2}=\emptyset$; for AP, we also assume there is a model $M_{0} \models T_{\mathfrak{m}, k}^{0}$ such that $M_{0} \subseteq M_{\ell}$ for $\ell=1,2, M_{1} \cap M_{2}=M_{0}$. Then consider the following model $N$. The domain of $N$ is $M_{1} \cup M_{2}$, for each unary predicate $X \in \tau_{k}$, let $X^{N}=X^{M_{1}} \cup X^{M_{2}}$, and let $R^{N}=R^{M_{1}} \cup R^{M_{2}}$. Thus $T_{\mathfrak{m}, k}$ exists.

Claim 2.19. For every $k_{*}<\omega$ the following holds: if $M \models T_{\mathfrak{m}, k_{1}}$, and $N \models T_{\mathfrak{m}, k_{2}}$, where $k_{1}, k_{2} \geq k_{*}$, and $\psi$ is a sentence of $\tau_{k_{*}}$ of length $\leq k_{*}$ (or just such that any subformula has $\leq k_{*}$ free variables), then $M \models \psi \Longleftrightarrow N \models \psi$.

Proof. Let $\mathcal{F}$ be defined by: $f \in \mathcal{F}=\mathcal{F}_{k_{*}}$ iff for some $k \leq k_{*}$ and $a_{0}, \ldots, a_{k-1} \in M$, $b_{0}, \ldots, b_{k-1} \in N$, we have that $f=\left\{\left(a_{\ell}, b_{\ell}\right): \ell<k\right\}$, and for every atomic $\varphi\left(x_{0}, \ldots, x_{k-1}\right) \in \mathcal{L}\left(\tau_{k_{*}}\right)$, we have that

$$
M \models \varphi\left[a_{0}, \ldots, a_{k-1}\right] \Longleftrightarrow N \models \varphi\left[b_{0}, \ldots, b_{k-1}\right] .
$$

(We could just as well replace "atomic" by "quantifier free.") Thus, $\mathcal{F}$ is a set of partial one to one functions $f$ from $M$ into $N$ such that $|\operatorname{dom}(f)| \leq k_{*}$, and clearly if $f \in \mathcal{F}$ and $A \subseteq \operatorname{dom}(f)$ then $f \nmid A \in \mathcal{F}$.

\footnotetext{
${ }^{6}$ We could have used predicates $P_{1}, P_{2}, P_{1, \eta}, P_{2, \rho}$ to emphasize the symmetry and to continue the notation of $\mathcal{T}_{1}, \mathcal{T}_{2}$, but chose $\mathcal{P}, \mathcal{Q}$ for readability.
} 
We claim that if $f \in \mathcal{F},|\operatorname{dom}(f)|<k_{*}$ and $a \in M, b \in N$ then there are $a^{\prime} \in M$, $b^{\prime} \in N$ such that $f \cup\left\{\left(a^{\prime}, b\right)\right\} \in \mathcal{F}$ and $f \cup\left\{\left(a, b^{\prime}\right)\right\} \in \mathcal{F}$. Suppose we are given $f=\left\{\left(a_{\ell}, b_{\ell}\right): \ell<k<k_{*}\right\}$ along with $a, b$. Since $k_{1}, k_{2} \geq k_{*}$ in the definition of $M$, $N$ are arbitrary, it will suffice to find $b^{\prime}$. Moreover, since either $a \in \mathcal{P}^{M}$ or $a \in \mathcal{Q}^{M}$, by symmetry (i.e. we can use dual( $\mathfrak{m})$ ) it suffices to consider the case $a \in Q^{M}$

Consider the sequence $\left\{a_{\ell}: \ell<k\right\}$ in $M$. Each $a_{\ell}$ is either in $\mathcal{Q}^{M}$ or $\mathcal{P}^{M}$. Renumbering, without loss of generality, there is $\ell_{*} \leq k$ such that $a_{\ell} \in \mathcal{P}^{M}$ for $\ell<\ell_{*}$ and $a_{\ell} \in \mathcal{Q}^{M}$ otherwise (so the corresponding fact is true for the $b_{\ell}$ 's in $N$ ). Also, without loss of generality, the sequence $\left\langle a_{\ell}: \ell<\ell_{*}\right\rangle$ is without repetition, and $a \notin\left\{a_{\ell}: \ell<\ell_{*}\right\}$, otherwise it is trivial. Since we have assumed that $a$, our new element, is in $\mathcal{Q}^{M}$, let $\eta \in \mathcal{T}_{1, k_{*}}$ be such that $a \in Q_{\eta}^{M}$. Looking now at $N$, recalling that $k_{2} \geq k_{*}$, let $\rho_{0}, \ldots, \rho_{\ell_{*}-1} \in \mathcal{T}_{2, k_{2}}$ be such that $b_{\ell} \in P_{\rho_{\ell}}^{N}$ for $\ell<\ell_{*}$. (It follows by our definition of $f \in \mathcal{F}$ that $a_{\ell} \in P_{\rho_{\ell} \uparrow k_{*}}^{N}$ for $\ell<\ell_{*}$.) It will suffice to find $b^{\prime} \in Q_{\eta}^{N}$ such that

$$
\left(a, a_{\ell}\right) \in R^{M} \Longleftrightarrow\left(b^{\prime}, b_{\ell}\right) \in R^{N} \text { for } \ell<\ell_{*} .
$$

The inequalities are easy so we ignore them. Note that the axioms for $T_{\mathfrak{m}, k_{*}}^{0} \subseteq$ $T_{\mathfrak{m}, k_{*}} \subseteq T_{\mathfrak{m}, k_{1}} \cap T_{\mathfrak{m}, k_{2}}$ in 2.15(4) imply that

$$
\left(a, a_{\ell}\right) \in R^{M} \Longrightarrow\left(\eta, \rho_{\ell}\left\lceil k_{*}\right) \in \mathcal{R}_{k_{*}} .\right.
$$

Thus, for equation (国), it will suffice to show that there is some $\eta^{\prime} \in \mathcal{T}_{1, k_{2}}$ such that $\eta \unlhd \eta^{\prime}$ and

$$
\left(a, a_{\ell}\right) \in R^{M} \Longrightarrow\left(\eta^{\prime}, \rho_{\ell}\right) \in \mathcal{R}_{k_{2}} \text {. }
$$

(It doesn't matter to us here whether the non-edges come from the randomness between leaves or from leaves with no edges between them.) Let us define by induction on $t \leq\left(k_{2}-k_{*}\right)$ a $\unlhd$-increasing sequence of elements $\eta_{t} \in \mathcal{T}_{1, k_{*}+t}$ such that $\eta_{0}=\eta, s \leq t \Longrightarrow \eta_{s} \unlhd \eta_{t}$, and $\left(a, a_{\ell}\right) \in R^{M} \Longrightarrow\left(\eta_{t}, \rho_{\ell}\left\lceil_{k_{*}+t}\right) \in \mathcal{R}_{k_{*}+t}\right.$. For $t=0$, this follows from equation (B). For $t \geq 0$, since $\ell_{*}<k_{*}$, we may apply the right extension axiom [2.6 (using $k_{*}+t, \eta_{t},\left\{\rho_{\ell} \uparrow_{k_{*}+t}: \ell<\ell_{*}\right\}$ here for $k, \eta,\{\rho\lceil k: \rho \in u\}$ there) and choose any one of the $\eta$ 's returned by that axiom to be $\eta_{t+1}$. Let $\eta^{\prime}=\eta_{k_{2}-k_{*}} \in \mathcal{T}_{1, k_{2}}$, and this completes the proof.

Corollary 2.20. When $\mathfrak{m}$ is a parameter, the sequence $\left\langle T_{\mathfrak{m}, k}: k<\omega\right\rangle$ converges. Moreover, for every formula $\varphi(\bar{x})$ of $\tau_{\mathfrak{m}}$, for some quantifier free $\psi(\bar{x})$, for every $k<\omega$ large enough, we have

$$
(\forall \bar{x})(\psi(\bar{x}) \equiv \varphi(\bar{x})) \in T_{\mathfrak{m}, k} .
$$

\footnotetext{
${ }^{7}$ Informally, here is the worry: $M$ is a fortiori a model of $T_{\mathfrak{m}, k_{*}}$, so the best quantifier-free $\tau_{k_{*}}$ information we have about the $a_{\ell}$ 's in $M$ is to know which leaf at level $k_{*}$ each of them belongs to (i.e. which $Q_{\eta}$ or $P_{\rho}$ for $\eta \in \mathcal{T}_{1, k_{*}}$ or $\rho \in \mathcal{T}_{2, k_{*}}$ ) and whether or not they connect via $R$. In the model $N$, the corresponding $b_{\ell}$ 's have the same quantifier-free $\tau_{k_{*}}$-type as their counterparts in $M$, but when looking for $b^{\prime}$ in $N$ we must consider an additional level of resolution, namely the leaf of each $b_{\ell}$ at at level $k_{2} \geq k_{*}$. For example, if $\left(Q_{\eta}^{M}, P_{\rho}^{M}, R^{M}\right), \operatorname{lgn}(\eta)=\operatorname{lgn}(\rho)=k_{*}$ form an infinite bipartite random graph in $M$, then for any finite set $u$ of elements of $Q_{\eta}^{M}$ there is $a \in P_{\rho}^{M}$ $R$-connecting to all of them. But suppose $f$ had mapped the elements of $u$ to elements of $Q_{\eta}^{N}$ which happened to span $Q_{\eta}^{N}\langle i\rangle$ for $i<\left|\operatorname{ims}_{\mathcal{T}_{1}}(\eta)\right|$. Then we could not find a corresponding $b^{\prime}$ in $N$. We solve this by limiting the size of sets $u$ in terms of $k_{*}$ and using the extension axioms.
} 
Conclusion 2.21. Let $\mathfrak{m}$ be a parameter and $T_{\mathfrak{m}}^{0}$ be the universal theory from 2.17, Then its model completion $T_{\mathfrak{m}}$ is well defined, eliminates quantifiers, and is equal to the limit of $\left\langle T_{\mathfrak{m}, k}: k<\omega\right\rangle$.

Lemma 2.22. Continuing in the context of Conclusion 2.21, the theory $T_{\mathfrak{m}}$ is simple and the only dividing comes from equality.

Proof. Work in the monster model for $T_{\mathfrak{m}}$. Observe that this theory has trivial algebraicity (and quantifier elimination). Let $\varphi(\bar{x} ; \bar{a})$ be a formula realized by some $\bar{b}$ with $\bar{b} \cap \bar{a}=\emptyset$. Without loss of generality, $\varphi(\bar{x}, \bar{y})$ decides instances of $R$ and equality between its variables, and implies that no two of its variables are equal. Suppose for a contradiction that $\varphi(\bar{x} ; \bar{a})$ divides, witnessed by the set of formulas $\left\{\varphi\left(\bar{x} ; \bar{a}^{i}\right): i<\left(2^{\aleph_{0}}\right)^{+}\right\}$being $k$-inconsistent, where $\left\langle\bar{a}^{i}: i<\left(2^{\aleph_{0}}\right)^{+}\right\rangle$is a (nontrivial) indiscernible sequence in the type of $\bar{a}$. Suppose $\operatorname{lgn}(\bar{x})=m$ and $\operatorname{lgn}(\bar{a})=\operatorname{lgn}(\bar{y})=$ $n$. [In fact, by basic properties of nonforking, it would suffice to consider $m=1$ and $T_{\mathfrak{m}, k}$ for each $k<k_{*}$, where the picture is very much like the random graph; this simplifies the proof. However, we give the general picture.]

Fix a sequence $\left\langle\bar{b}^{i}: i<\left(2^{\aleph_{0}}\right)^{+}\right\rangle$of $m$-tuples such that $\models \varphi\left[\bar{b}^{i}, \bar{a}^{i}\right]$ and $\bar{b}^{i} \cap \bar{a}^{i}=\emptyset$ for each $i$. Write $\bar{b}^{i}=\left\langle b_{0}^{i}, \ldots, b_{m-1}^{i}\right\rangle$ and $\bar{a}^{i}=\left\langle a_{0}^{i}, \ldots, a_{n-1}^{i}\right\rangle$. Since $\left(2^{\aleph_{0}}\right)^{+}$is regular, we may assume the type of $b_{j}^{i}$ over the empty set does not depend on $i$, and also (by definition of indiscernible) that the type of $a_{t}^{i}$ over the empty set does not depend on $i$. [The "leaf" to which the $\ell$-th element of the tuple $\bar{b}^{i} \frown \bar{a}^{i}$ belongs is constant as we vary $i$.] That is, for $j<m$ :

- if $\models \mathcal{Q}\left(b_{j}^{i}\right)$, then there is $\eta_{*}=\eta_{*}(j) \in \lim \left(\mathcal{T}_{1}\right)$ such that $\models Q_{\eta_{*}(j) \ell \ell}$ for all $\ell<\omega$, where $\eta_{*}(j)$ depends on $j$ but not on $i$.

- if $\models \mathcal{P}\left(b_{j}^{i}\right)$, then there is $\nu_{*}(j) \in \lim \left(\mathcal{T}_{2}\right)$ such that $\models P_{\nu_{*}(j)\lceil\ell}$ for all $\ell<\omega$, where $\nu_{*}(j)$ depends on $j$ but not on $i$.

Likewise, for $t<n$,

- if $\models \mathcal{Q}\left(a_{t}^{i}\right)$, then there is $\eta_{*}=\eta_{*}(t) \in \lim \left(\mathcal{T}_{1}\right)$ such that $\models Q_{\eta_{*}(t) \mid \ell}$ for all $\ell<\omega$, where $\eta_{*}(t)$ depends on $t$ but not on $i$.

- if $\models \mathcal{P}\left(a_{t}^{i}\right)$, then there is $\nu_{*}(t) \in \lim \left(\mathcal{T}_{2}\right)$ such that $\models P_{\nu_{*}(t) \mid \ell}$ for all $\ell<\omega$, where $\nu_{*}(t)$ depends on $t$ but not on $i$.

Recall that by quantifier elimination, the only information $\varphi$ can specify about the relation of any given $x_{j}$ to the other $x$ or $y$ variables involves the unary predicates, the relation $R$, and equality.

Now we shall choose by induction on $j<m$ elements $b_{j}^{*}$ such that the sequence $\left\langle b_{j}^{*}: j<m\right\rangle$ realizes $\left\{\varphi\left(\bar{x} ; \bar{a}^{i}\right): i<\left(2^{\aleph_{0}}\right)^{+}\right\}$, and this contradiction will finish the proof. At stage $j$, we'll want to keep track of which elements of $\left\{a_{t}^{i}: i<\left(2^{\aleph_{0}}\right)^{+}, t<\right.$ $n\} \cup\left\{b_{s}^{*}: s<j\right\}$ are in $\mathcal{Q}$ and which are in $\mathcal{P}$ (keeping in mind that the partition of the first set depends only on $t$ ). Let

$$
A_{\mathcal{Q}}^{j}=\left\{a_{t}^{i}: i<\left(2^{\aleph_{0}}\right)^{+}, t<n, \models \mathcal{Q}\left(a_{t}^{i}\right)\right\} \cup\left\{b_{s}^{*}: s<j, \models \mathcal{Q}\left(b_{s}^{*}\right)\right\}
$$

and likewise let

$$
A_{\mathcal{P}}^{j}=\left\{a_{t}^{i}: i<\left(2^{\aleph_{0}}\right)^{+}, t<n, \models \mathcal{P}\left(a_{t}^{i}\right)\right\} \cup\left\{b_{s}^{*}: s<j, \models \mathcal{P}\left(b_{s}^{*}\right)\right\} .
$$

Thus, for each $j<m$, there are two cases. If $\models \mathcal{Q}\left(b_{j}^{i}\right)$ (for some, equivalently every, $i$ ) then it suffices to choose $b_{j}^{*}$ such that:

- $\left\{b_{j}^{*}\right\} \cap A_{\mathcal{Q}}^{j}=\emptyset$, i.e. $b_{j}^{*}$ is not equal to any other element in $\mathcal{Q}$ under consideration; and 
- $b_{j}^{*}$ satisfies the appropriate pattern of $R$-edges and non-edges over the elements of $A_{\mathcal{P}}^{j}$ as specified by $\varphi$, i.e.

- if $a_{t}^{i} \in A_{\mathcal{P}}^{j}$ and $\varphi \vdash R\left(x_{j}, y_{t}\right)$, then $R\left(b_{j}^{*}, a_{t}^{i}\right)$

- if $a_{r}^{i} \in A_{\mathcal{P}}^{j}$ and $\varphi \vdash \neg R\left(x_{j}, y_{r}\right)$, then $R\left(b_{j}^{*}, a_{r}^{i}\right)$

- if $s<j$ and $b_{s}^{*} \in A_{\mathcal{P}}^{j}$ and $\varphi \vdash R\left(x_{s}, x_{j}\right)$, then $R\left(b_{s}^{*}, b_{j}^{*}\right)$

- if $s<j$ and $b_{s}^{*} \in A_{\mathcal{P}}^{j}$ and $\varphi \vdash \neg R\left(x_{s}, x_{j}\right)$, then $\neg R\left(b_{s}^{*}, b_{j}^{*}\right)$.

We know from the universal theory $T_{\mathfrak{m}}^{0}$ that the consistency of these demands relies on the predicates of the elements and the equalities between them, and nothing else 8 Of course, $\varphi$ need not decide all predicates a priori, but on our subsequence, we ensured this information is effectively decided, constant across $i$, and consistent with the pattern of edges implied by $\varphi$, as witnessed by the consistency of each $\varphi\left(\bar{x}, \bar{a}^{i}\right)$; meanwhile, our choice of indiscernible sequence and inductive hypothesis ensure no trouble is provided by equality. So we can carry the inductive step.

If $\models \mathcal{P}\left(b_{j}^{i}\right)$ (for some, equivalently every, $i$ ) then it suffices to choose $b_{j}^{*}$ satisfying the parallel conditions with $\mathcal{Q}$ and $\mathcal{P}$ reversed, and the justification is the same.

This completes the proof.

Conclusion 2.23. Let $\mathfrak{m}$ be a parameter and $T_{\mathfrak{m}}^{0}$ be the universal theory from 2.17 . Then its model completion $T_{\mathfrak{m}}$ exists, eliminates quantifiers, is simple unstable, and the only dividing comes from equality.

We will continue with a description of the models and types of $T_{\mathfrak{m}}$ in 4 after some discussion.

\section{A DARK WOODS}

In this primarily expository section we make some motivating and organizing remarks about the new theories of $\$ 2$.

Recall that " $R$ acts as an (infinite, model theoretic) bipartite random graph between the sets $A, B$ " is shorthand for: for any two disjoint finite subsets $A_{0}, A_{1}$ of $A$, there is an element $b \in B$ which has an $R$-edge to all elements of $A_{0}$ and to no elements of $A_{1}$, and for any two disjoint finite subsets $B_{0}, B_{1}$ of $B$, there is an element $a \in A$ which has an $R$-edge to all elements of $B_{0}$ and to no elements of $B_{1}$.

Let's begin with some very simple examples, which are too basic to satisfy the definitions of $\$ 2$ outright (the signatures are finite) but illustrative nonetheless.

Example 3.1. Suppose $\tau$ includes unary predicates $\mathcal{Q}$ and $\mathcal{P}$, a binary relation $R$, and nothing else. Suppose $T_{0}$ asserts that $\mathcal{Q}$ and $\mathcal{P}$ partition the domain, and that $R \subseteq \mathcal{Q} \times \mathcal{P}$. Then the model completion $T$ of $T_{0}$ exists. In models $M$ of $T$, both $\mathcal{P}^{M}$ and $\mathcal{Q}^{M}$ are infinite, and partition the domain. $R$ acts as a model theoretic bipartite random graph between $\mathcal{Q}^{M}$ and $\mathcal{P}^{M}$. (There are no instances of $R$ within $\mathcal{P}^{M}$ or $\mathcal{Q}^{M}$.)

Example 3.2. Suppose $\tau$ includes unary predicates $\mathcal{Q}, Q_{0}, Q_{1}, Q_{2}, \mathcal{P}, P_{0}, P_{1}, P_{2}$, a binary relation $R$, and nothing else. Suppose $T_{0}$ asserts that $\mathcal{Q}$ and $\mathcal{P}$ partition

\footnotetext{
${ }^{8}$ To be clear, recall that if two nodes $\eta, \nu$ of finite height are connected in the template trees, then elements of $Q_{\eta}, P_{\nu}$ are free to be related or unrelated by $R$, whereas if $\eta, \nu$ are not connected, then all corresponding instances of $R$ are forbidden. If $\eta_{*} \in \lim \left(\mathcal{T}_{1}\right)$ and $\nu_{*} \in \lim \left(\mathcal{T}_{2}\right)$ and $Q_{\eta_{*} \mid \ell}(a)$ for $\ell<\omega$ and $P_{\nu_{*} \mid \ell}(b)$ for $\ell<\omega$ and $\left(\eta_{*}\left\lceil\ell, \nu_{*}\lceil\ell) \in \mathcal{R}_{\ell}\right.\right.$ for $\ell<\omega$, then there are a priori no constraints on whether or not $R$ holds between $a$ and $b$.
} 
the domain; that $Q_{0}, Q_{1}, Q_{2}$ partition $\mathcal{Q}$; that $P_{0}, P_{1}, P_{2}$ partition $\mathcal{P}$; and that $R \subseteq$ $\mathcal{Q} \times \mathcal{P}$. Suppose that in addition $T_{0}$ asserts that:

- there are no $R$-edges between $Q_{0}$ and $P_{1}$, and

- there are no $R$-edges between $Q_{1}$ and $P_{2}$.

Then the model completion $T$ of $T_{0}$ exists. In a model $M$ of $T$, we have that $Q_{0}^{M}$, $Q_{1}^{M}, Q_{2}^{M}, P_{0}^{M}, P_{1}^{M}, P_{2}^{M}$ are all infinite, and partition the domain. There are no instances of $R$ between $Q_{0}^{M}$ and $P_{1}^{M}$, between $Q_{1}^{M}$ and $P_{2}^{M}$, or within $\mathcal{Q}^{M}$ or within $\mathcal{P}^{M}$. Notice that $R$ acts as a model-theoretic bipartite random graph between $Q_{i}^{M}$ and $P_{j}^{M}$ for $(i, j) \in\{0,1,2\} \times\{0,1,2\} \backslash\{(0,1),(1,2)\}$. What's more, $R$ acts as a model-theoretic bipartite random graph between $Q_{0}^{M} \cup Q_{1}^{M}$ and $P_{1}^{M}$, and also between $Q_{1}^{M} \cup Q_{2}^{M}$ and $P_{0}^{M} \cup P_{1}^{M}$. Indeed, $R$ acts as a model-theoretic bipartite random graph between $\bigcup_{i \in u} Q_{i}^{M}$ and $\bigcup_{j \in v} P_{j}^{M}$ whenever $u \subseteq\{0,1,2\}, v \subseteq\{0,1,2\}$ and none of the pairs $Q_{i}, P_{j}$ for $(i, j) \in u \times v$ have their $R$-edges forbidden by $T_{0}$.

Observe that a simple way to encode the information at the end of Example 3.2 could be to consider a kind of bipartite "reduced graph" between the indices for "leaves," here $\{0,1,2\}$ and $\{0,1,2\}$, where we put a symbolic edge between $i$ and $j$ if and only if $T_{0}$ does not forbid $R$-edges between $Q_{i}$ and $P_{j}$. Then we can summarize Example 3.2 (slightly abusing notation by not referencing a model) by saying $R$ acts as a model-theoretic bipartite random graph between $\bigcup_{i \in u} Q_{i}$ and $\bigcup_{j \in v} P_{j}$ precisely when the restriction of our bipartite reduced graph to the vertices in $u$ and $v$ is complete 9

We now point out that in the more general context of parameters and the larger signatures of 92 , such "reduced graphs" on the "leaves" likewise give a nice picture of our theories $T_{\mathfrak{m}}$, and can be phrased naturally in terms of the template edges $\mathcal{R}$. The next two definitions allow us to compare what we are calling the reduced graph to the $R$-graph in a model of the theory, starting with the case where we restrict to nodes of a fixed finite height $k$.

Definition 3.3. Suppose we are given a parameter $\mathfrak{m}$, a finite $k$, and nonempty sets $V \subseteq \mathcal{T}_{1, k}$ and $W \subseteq \mathcal{T}_{2, k}$. Let

$$
H_{k}(V, W)=\left(V, W, \mathcal{R}_{k}\lceil V \times W) .\right.
$$

Definition 3.4. Suppose we are given a parameter $\mathfrak{m}$ thus $T_{\mathfrak{m}}$, a finite $k$, a model $M \models T_{\mathfrak{m}}$, and nonempty sets $V \subseteq \mathcal{T}_{1, k}$ and $W \subseteq \mathcal{T}_{2, k}$. Let

$$
G_{k}(V, W)=G_{k}(V, W)[M]=\left(\bigcup_{\eta \in V} Q_{\eta}^{M}, \bigcup_{\eta \in W} P_{\rho}^{M}, R \uparrow\left(\bigcup_{\eta \in V} Q_{\eta}^{M} \times \bigcup_{\eta \in W} P_{\rho}^{M}\right)\right) .
$$

Remark 3.5. The phrase "reduced graph" may bring to mind Szemerédi's regularity lemma for graphs, where recall that a given finite graph is partitioned into clusters in such a way that between most pairs of clusters the edges are distributed $\epsilon$-uniformly. There one may define a reduced graph (see [11] p. 306), for instance by taking one vertex for each cluster, and with an edge between two points whose associated clusters are $\epsilon$-regular with density $\epsilon$-bounded away from 0 (and if desired, 1). Such a reduced graph doesn't only record the "generic interaction" of a given pair of clusters, but also entails that there is a certain further genericity in the interaction of more than two clusters, e.g. if three points in the reduced graph form

\footnotetext{
${ }^{9}$ Of course, the restriction to $\{i\}$ and $\{j\}$ when there is a symbolic edge between $i$ and $j$ is just a special case of a complete bipartite graph.
} 
a triangle, we should be able to get many triangles on three vertices spanning the associated clusters in the original graph. A certain analogue of this in our setting is in the comment after Example 3.2 about complete bipartite graphs.

The next definition gives the full analogue for the countable height trees we really use in $\mathfrak{m}$ and $T_{\mathfrak{m}}$. The word virtual reflects that the objects are generally not definable, though they may be type-definable. Though what we call " $\mathcal{R}$ " is not definable in 3.6. the edge relation in 3.7 is simply $R^{M}$.

Definition 3.6 (Virtual reduced graph). Let $\mathfrak{m}=\left(\mathcal{T}_{1}, \mathcal{T}_{2}, \mathcal{R}\right)$ be a parameter.

(1) Define $\mathcal{R}^{\infty}=\left\{(\rho, \eta):(\rho, \eta) \in \lim \left(\mathcal{T}_{1}\right) \times \lim \left(\mathcal{T}_{2}\right)\right.$ and $\left(\rho\lceil k, \eta \uparrow k) \in \mathcal{R}_{k}\right.$ for all $k<\omega\}$.

(2) Then for any nonempty $V \subseteq \lim \left(\mathcal{T}_{1}\right)$ and $W \subseteq \lim \left(\mathcal{T}_{2}\right)$, define the virtual reduced graph

$$
H^{\infty}(V, W)
$$

to be the bipartite graph $\left(V, W, \mathcal{R}^{\infty}\right)$.

That is, 3.6 defines a bipartite graph whose vertices are the leaves of $\mathcal{T}_{1}$ on the left and the leaves of $\mathcal{T}_{2}$ on the right and where $(\eta, \nu)$ is an edge if and only if $\left(\eta\lceil n, \nu \uparrow n) \in \mathcal{R}_{n}\right.$ for all $n<\omega$. (2) gives various induced subgraphs.

Definition 3.7 (Virtual graph). Continuing in the notation of [3.6, suppose we are given any model $M \models T_{\mathfrak{m}}$.

(1) For any $V \subseteq \lim \left(\mathcal{T}_{1}\right)$, let the expression $Q_{V}^{\infty}=Q_{V}^{\infty}[M]$ denote

$\left\{a \in \operatorname{dom}(M):\right.$ for some $\eta \in V, M \models Q_{\eta \uparrow k}(a)$ for all $\left.k<\omega\right\}$.

In particular, for any $\eta \in \lim \left(\mathcal{T}_{1}\right), Q_{\{\eta\}}^{\infty}=Q_{\{\eta\}}^{\infty}[M]$ denotes the subset of $M$ realizing the type $\left\{Q_{\eta \uparrow k}(x): k<\omega\right\}$.

(2) Likewise for any $W \subseteq \lim \left(\mathcal{T}_{2}\right)$, let the expression $Q_{W}^{\infty}=Q_{W}^{\infty}[M]$ denote

$$
\left\{b \in \operatorname{dom}(M): \text { for some } \rho \in W, M \models P_{\eta \uparrow k}(b) \text { for all } k<\omega\right\} \text {. }
$$

In particular, for any $\rho \in \lim \left(\mathcal{T}_{2}\right), P_{\{\rho\}}^{\infty}=P_{\{\rho\}}^{\infty}[M]$ denotes the subset of $M$ realizing the type $\left\{P_{\rho \nmid k}(x): k<\omega\right\}$.

(3) For any nonempty $V \subseteq \lim \left(\mathcal{T}_{1}\right), W \subseteq \lim \left(\mathcal{T}_{2}\right)$, let the virtual graph

$$
G^{\infty}(V, W)=G^{\infty}(V, W)[M]
$$

be the bipartite graph

$$
\left(Q_{V}^{\infty}, P_{W}^{\infty}, R^{M}\left\lceil Q_{V}^{\infty} \times P_{W}^{\infty}\right) .\right.
$$

That is, 3.7 defines a bipartite graph from $M$ whose vertices are elements of $Q$ belonging to certain "leaves" on the left and the elements of $P$ belonging to certain other "leaves" on the right, along with the edge relation given by $R$.

Discussion 3.8. We defer to $\S 2$, 团 for details. Given a parameter $\mathfrak{m}$ :

a) The structure of models of $T_{\mathfrak{m}}$ is in some sense simple: in the language of 3.6 and 3.7, in a sufficiently saturated 10 model $M \models T_{\mathfrak{m}}$, given any nonempty $V \subseteq \lim \left(\mathcal{T}_{1}\right)$ and $W \subseteq \lim \left(\mathcal{T}_{2}\right)$, if $H^{\infty}(V, W)$ is a complete graph, then $G^{\infty}(V, W)[M]$ is an infinite bipartite random graph, and if $H^{\infty}(\{\eta\},\{\rho\})$ is empty, then so is $G^{\infty}(\{\eta\},\{\rho\})[M]$. (Letting $V, W$ vary, these two facts together are enough to put together the whole picture.)

\footnotetext{
${ }^{10}$ For simplicity, to ensure all countable intersections of predicates are nonempty.
} 
b) In any $\aleph_{1}$-saturated model $M$ of $T_{\mathfrak{m}}$, e.g. in a regular ultrapower, for any leaves $\eta \in \lim \left(\mathcal{T}_{1}\right), \rho \in \lim \left(\mathcal{T}_{2}\right)$, the sets $Q_{\{\eta\}}^{\infty}, P_{\{\rho\}}^{\infty}$ will be infinite, and will have among them the infinite empty or random graph structure just mentioned. We will see in detail in $\$ 4$ that for $\lambda^{+}$-saturation, we will want each such $Q_{\{\eta\}}^{\infty}$ and each such $P_{\{\rho\}}^{\infty}$ to have size at least $\lambda^{+}$, and moreover, for every $V \subseteq \lim \left(\mathcal{T}_{1}\right)$ and $W \subseteq \lim \left(T_{2}\right)$ such that $H^{\infty}(V, W)$ is a complete graph $11, G^{\infty}(V, W)$ is $\lambda^{+}$-saturated as a bipartite random graph, i.e.

(i) for any two disjoint subsets $A, B$ of $P_{W}^{\infty}$ of size $\lambda$, and any $\eta \in V$, there is $c \in Q_{\{\eta\}}^{\infty}$ which $R$-connects to all $a \in A$, no $b \in B$.

(ii) the parallel reversing $V, W$ and $Q, P$.

In what follows, we will focus on $\mathfrak{m}$ such that $\mathfrak{m}=\operatorname{dual}(\mathfrak{m})$; so by symmetry, it will be enough to handle one of (i), (ii), and as we will see in 4.8 and 5.6 below, it will generally be enough to realize partial positive $R$-types.

Where does the potential for widely differing complexity arise? The following informal discussion may help the reader anticipate or follow the proof.

Why might these theories interact with ultrapowers in an interesting way? In an ultrapower of a model of $T_{\mathfrak{m}}$, elements which are "on average" part of the same leaf may nonetheless appear, when projected to a given index model, to be in too many different predicates at a given height $k$, blocking realization of the type in that index model when splitting is constrained. Both the size of allowed splitting at a given height in a given tree (and, by extension, the level functions) come into play, which in turn reflect the degrees of the vertices in the reduced graphs $H_{k}$.

Why might different parameters $\mathfrak{m}, \mathfrak{n}$ produce theories $T_{\mathfrak{m}}, T_{\mathfrak{n}}$ which look different to ultrapowers? The structure of each theory $T_{\mathfrak{m}}$ will reflect its sequence of "reduced graphs," based on the finite bipartite graphs $\mathcal{R}_{i}=\mathcal{R}_{i}(\mathfrak{m})$, and the related level function $\xi=\xi(\mathfrak{m})$, which is active at infinitely many $n \in \omega$. When $\xi$ is not active, $\mathcal{R}_{i+1}$ gives essentially no new information beyond $\mathcal{R}_{i}$. A natural way to vary the $\mathcal{R}_{i}$ 's will be to consider a single fast-growing sequence of sparse graphs $\left\langle E_{i}: i<\omega\right\rangle$, choose many level functions which are independent in a natural sense, and build for each such $\xi$ a theory whose $\mathcal{R}_{i}$ essentially copies $E_{i}$ at active levels and copies a complete bipartite graph of the right size at lazy levels. This allows us to vary the sequences of reduced graphs in a very clear way. Remarkably, these differences are detected in a very strong sense both by the theories themselves and by ultrafilters. To prove this will also require an advance in ultrafilter construction.

Remark 3.9. To make these suggestions precise will, of course, require the rest of the paper; but notice that the construction already suggests many further modifications and interesting future directions, some discussed at the end of the paper.

\section{Models OF $T_{\mathfrak{m}}$}

In this section we analyze the types over a model $M$ of $T_{\mathfrak{m}}$, which will help later in dealing with saturation.

Note that we use almost nothing about the level functions in this section; we just need the extension axioms to ensure a minimum increase in the edges. The level

\footnotetext{
${ }^{11}$ What about other $W$ s? It can't hurt, but won't add anything: see last line of proof of 4.6
} 
functions operate at a different scale in the sense that they control variations in the number of edges well beyond the minimum established by the extension axioms, and will mainly play a role in later sections, where we try to compare theories.

Convention 4.1. In this section, $\mathfrak{m}$ is an arbitrary but fixed parameter, and $M$ is a model of $T_{\mathfrak{m}}$.

For the purposes of our analysis, because of the symmetry of $\mathfrak{m}$, it will suffice to deal with types $q(x)$ in one free variable $x$ which describe an element on the left, i.e. $q(x) \vdash \mathcal{Q}(x)$. Note that any such type, being complete, will specify that $Q_{\rho \nmid n}(x)$ for some $\rho \in \lim \left(\mathcal{T}_{1}\right)$ and all $n<\omega$.

Here is some notation for the connections made along the way by a leaf $\rho$.

Definition 4.2. For $\rho \in \lim \left(\mathcal{T}_{1}\right)$, we define:

(1) $\mathcal{S}_{\rho}=\left\{\nu:\right.$ for some finite $n, \nu \in \mathcal{T}_{2, n}$ and $\left(\rho\lceil n, \nu) \in \mathcal{R}_{n}\right\}$.

(2) $\lim \left(\mathcal{S}_{\rho}\right)=\left\{\eta \in \lim \left(\mathcal{T}_{2}\right): \eta\left\lceil n \in \mathcal{S}_{\rho}\right.\right.$ for $\left.n<\omega\right\}$ $=\left\{\eta \in \lim \left(\mathcal{T}_{2}\right):(\rho, \eta) \in \mathcal{R}^{\infty}\right\}$.

Observation 4.3. Recalling 2.4, if $\rho \in \lim \left(\mathcal{T}_{1}\right), \mathcal{S}_{\rho}$ is a subtree of $\mathcal{T}_{2}$ with no maximal node.

Recall some notation from the previous section. For $\eta \in \lim \left(\mathcal{T}_{2}\right), P_{\{\eta\}}^{\infty}=P_{\{\eta\}}^{\infty}[M]$ denotes the elements of $M$ which are "in the leaf" corresponding to $\eta$, and the corresponding notation for $\rho \in \lim \left(\mathcal{T}_{1}\right)$ is $Q_{\{\rho\}}^{\infty}=Q_{\{\rho\}}^{\infty}[M]$. We had likewise defined $P_{V}^{\infty}$, $Q_{W}^{\infty}$ in 3.7 which also depend on $M$. Recall the virtual reduced graph $H^{\infty}(V, W)$ from 3.6. and the virtual graph $G^{\infty}(V, W)=G^{\infty}(V, W)[M]$ from 3.7.

Observation 4.4. For any $\rho \in \lim \left(\mathcal{T}_{1}\right)$ and $W \subseteq \lim \left(\mathcal{T}_{2}\right)$ such that $H^{\infty}(\{\rho\}, W)$ is complete, we have that $\lim \left(\mathcal{S}_{\rho}\right) \supseteq W$, in other words, $\mathcal{S}_{\rho}$ contains all proper initial segments of elements of $W$.

Claim 4.5. Suppose $\rho \in \lim \left(\mathcal{T}_{1}\right)$ and write $W=\lim \left(\mathcal{S}_{\rho}\right)$. For any

$$
A, B \subseteq P_{W}^{\infty}[M] \text { with } A \cap B=\emptyset
$$

the following set of formulas is a non-algebraic partial type of $M$ :

$$
\left\{Q_{\rho \nmid n}(x): n<\omega\right\} \cup\{R(x, a): a \in A\} \cup\{\neg R(x, b): b \in B\} .
$$

Proof. Consider a finite subset, which without loss of generality is of the form:

$$
\left\{Q_{\rho \nmid n}(x): n<k\right\} \cup\left\{R\left(x, a_{0}\right), \ldots, R\left(x, a_{\ell-1}\right)\right\} \cup\left\{\neg R\left(x, b_{0}\right), \ldots, \neg R\left(x, b_{r-1}\right)\right\} .
$$

Each of the elements $a_{i}, b_{j}$ has a leaf in $M$ : let's suppose that for each $i<\ell$, $\eta_{i}$ is such that $M \models P_{\eta_{i} \mid n}\left(a_{i}\right)$ for $n<\omega$ and for each $j<r, \nu_{j}$ is such that $M \models P_{\nu_{j} \mid n}\left(b_{j}\right)$ for $n<\omega$, though these leaves need not necessarily be distinct. By our choice of $A, B$ [that is, by the definition of $W$ ], we have that for any finite level, and in particular for $k$,

$$
\left(\rho \left\lceilk , \eta _ { i } \lceil k ) \in \mathcal { R } _ { k } \text { and } \left(\rho \left\lceilk, \nu_{j}\lceil k) \in \mathcal{R}_{k}\right.\right.\right.\right.
$$

for $i<\ell, j<r$. Thus the following sentence belongs to $T_{\mathfrak{m}, k}$ :

$$
(\exists x)\left(\bigwedge_{i<\ell} R\left(x, a_{i}\right) \wedge \bigwedge_{j<r} R\left(x, b_{j}\right)\right) .
$$

By 2.19, this remains true all the way to $T_{\mathfrak{m}}$. Since this shows an arbitrary finite subset is consistent, we finish the proof. 
Corollary 4.6. Suppose $\rho \in \lim \left(\mathcal{T}_{1}\right)$ and write $W=\lim \left(\mathcal{S}_{\rho}\right)$. For any $A, B \subseteq M$ with $A \cap B=\emptyset$ we have that

$$
r(x)=\left\{Q_{\rho \nmid n}(x): n<\omega\right\} \cup\{R(x, a): a \in A\} \cup\{\neg R(x, b): b \in B\}
$$

is a non-algebraic partial type of $M$ if and only if $A \subseteq P_{W}^{\infty}[M]$.

Proof. Suppose we denote $A_{0}=A \cap P_{W}^{\infty}$ and $B_{0}=B \cap P_{W}^{\infty}$. Let

$$
r_{0}=\left\{Q_{\rho \mid n}(x): n<\omega\right\} \cup\left\{R(x, a): a \in A_{0}\right\} \cup\left\{\neg R(x, b): b \in B_{0}\right\} .
$$

Claim 4.5 tells us that $r_{0}$ is a partial type.

First consider any element $b \in B \backslash B_{0}$. If $b \in Q^{M}$, then $\neg R(x, b)$ follows by definition as $R^{M} \subseteq Q^{M} \times P^{M}$. If $b \in P^{M}$, then because $M$ is a model, there is some $\eta$ such that $b \in P_{\eta \uparrow n}^{M}$ for all $n<\omega$. If $(\rho, \eta) \notin \mathcal{R}^{\infty}$, then there is some $n<\omega$ for which $\left(\eta\left\lceil n, \rho\lceil n) \notin \mathcal{R}_{n}\right.\right.$, which translates to

$$
M \models(\forall x)(\forall y)\left(Q_{\eta\lceil n}(x) \wedge P_{\rho\lceil n}(y) \Longrightarrow \neg R(x, y)\right)
$$

and so $r_{0} \vdash \neg R(x, b)$.

Finally, suppose that $A \backslash A_{0}$ is nonempty, and let $a$ be any one of its elements. Let $\eta$ be such that $a \in P_{\eta\lceil n}^{M}$ for all $n<\omega$. By definition of $A_{0},(\rho, \eta) \notin \mathcal{R}^{\infty}$, so it follows from the previous paragraph that $r_{0} \vdash \neg R(x, a)$. Thus $r$ is consistent if and only if $A \backslash A_{0}=\emptyset$.

Note that this proof shows that if $r$ is consistent, $r_{0} \vdash r$.

The next claim justifies restricting our saturation arguments to considering types of a very simple form.

Definition 4.7. We say a model $M$ of $T_{\mathfrak{m}}$ is weakly $\lambda^{+}$-saturated when:

(1) "the leaves are large": for any $\eta \in \lim \left(\mathcal{T}_{1}\right), \mid\left\{a \in M: Q_{\eta \mid n}^{M}(a)\right.$ for all $n<\omega\} \mid>\lambda$, and likewise for $\nu \in \lim \left(\mathcal{T}_{2}\right)$.

(2) if $c \in Q^{M}$ then $\left\{b:(c, b) \in R^{M}\right\} \subseteq P^{M}$ has cardinality $>\lambda$.

(3) the dual to the previous line: if $b \in P^{M}$ then $\left\{c:(c, b) \in R^{M}\right\} \subseteq Q^{M}$ has cardinality $>\lambda$.

Claim 4.8 (A basic form for non-algebraic types). Suppose $M$ is weakly $\lambda^{+}$. saturated. For any $C \subseteq M,|C| \leq \lambda$, and $q \in \mathbf{S}_{1}(C)$ such that $q(x) \vdash Q(x)$, there exist $\rho, W \subseteq \lim \left(\mathcal{S}_{\rho}\right), A \subseteq P_{W}^{\infty}[M], B \subseteq P^{M}$ with $A \cap B=\emptyset$ and $|A|+|B| \leq \lambda$, such that writing

$$
r(x)=\left\{Q_{\rho \nmid n}(x): n<\omega\right\} \cup\{R(x, a): a \in A\} \cup\{\neg R(x, b): b \in B\} .
$$

we have $r(x) \vdash q(x)$. We may also ask that $|A|,|B|=\lambda$.

Proof. By hypothesis, every "leaf" $Q_{\{\eta\}}^{\infty}[M]$ or $P_{\{\rho\}}^{\infty}[M]$ has size $>\lambda$.

To start, let $\rho \in \lim \left(\mathcal{T}_{1}\right)$ be such that $q(x) \vdash Q_{\rho\lceil n}(x)$ for all $n<\omega$, which exists as $q$ is a complete type. Define $W:=\lim \left(\mathcal{S}_{\rho}\right), A_{0}:=\{c \in C: q \vdash R(x, c)\} \cap P_{W}^{\infty}[M]$, and $B_{0}:=\{c \in C: q \vdash \neg R(x, c)\} \cap P_{W}^{\infty}[M]$. Let

$$
r_{0}(x)=\left\{Q_{\rho \nmid n}(x): n<\omega\right\} \cup\left\{R(x, a): a \in A_{0}\right\} \cup\left\{\neg R(x, b): b \in B_{0}\right\} \subseteq q(x) .
$$

Clearly $r_{0}$ is consistent and implies at least the restriction of $q(x)$ to the given unary predicates and to all positive and negative instances of $R(x, y)$ on $P_{W}^{\infty}[M] \cap \operatorname{dom}(q)$. Since $T_{\mathfrak{m}}=T h(M)$ eliminates quantifiers, it suffices to consider quantifier-free formulas. Let us check that by possibly increasing $A_{0}, B_{0}$ by no more than $\lambda$ elements, we can ensure that any formulas of the following kinds which belong to 
our original $q$ are also implied. Along the way, we remark on the consequences for this choice of larger partial type $r$, formally defined in $(\star)$ below.

(a) $x \neq c \in q$

If $c \in \mathcal{P}^{M}$, then this follows from $\mathcal{Q}(x)$. Let $\left\langle c_{\alpha}: \alpha<\kappa\right\rangle$ enumerate all elements of $\mathcal{Q}^{M}$ such that " $x \neq c_{\alpha}$ " is implied by $q(x)$, or equivalently belongs to $q(x)$. For each $\alpha<\kappa$, choose some $b_{\alpha} \notin A_{0}$ such that $M \models$ $R\left(c_{\alpha}, b_{\alpha}\right)$. We can do this because each $R\left(c_{\alpha}, x\right)$ defines an infinite subset of $M$ which is by hypothesis and Definition 4.7 of size at least $\lambda^{+}$. Adding $\left\{b_{\alpha}: \alpha<\kappa\right\}$ to $B_{0}$ to form $B_{1}$ will not raise its size above $\lambda$ and will mean that $r(x) \vdash x \neq c_{\alpha}$ for each $\alpha<\kappa$. Let $A_{1}:=A_{0}$.

(b) $\neg Q_{\nu}(x) \in q$, for $\nu \notin\left\{\rho\lceil n: n<\omega\}\right.$, or $\neg P_{\eta}(x) \in q$.

This follows from our assumption that $\left\{Q_{\rho \nmid n}(x): n<\omega\right\} \subseteq r_{0}$.

(c) $\neg R(x, b)$, for any $b \in B_{1} \backslash P_{W}^{\infty}[M]$.

For any $b \notin P_{W}^{\infty}[M]$, there is a finitary reason for the non-membership, i.e. there is $k<\omega$ and $\nu \in \mathcal{T}_{2, k}$ such that $(\rho \uparrow k, \nu) \notin \mathcal{R}_{k}$ and $b \in P_{\nu}^{M}$. Then $T_{\mathfrak{m}} \vdash(\forall x)(\forall y)\left(Q_{\rho \uparrow k}(x) \wedge P_{\nu}(y) \Longrightarrow \neg R(x, y)\right)$. As $r(x) \vdash Q_{\rho \uparrow k}(x)$, necessarily $r(x) \vdash \neg R(x, b)$.

Define $A=A_{1}, B=B_{1}$, and define

$$
(\star) r(x)=\left\{Q_{\rho \nmid n}(x): n<\omega\right\} \cup\{R(x, a): a \in A\} \cup\{\neg R(x, b): b \in B\} .
$$

This is a partial type of size $\leq \lambda$ and implies $q(x)$ as desired.

At this point, if we would like to ensure $|A|=|B|=\lambda$, there is no harm in choosing disjoint sets $A^{\prime}, B^{\prime}$ of size $\lambda$ from $P_{W}^{\infty}[M] \backslash\left(A_{1} \cup B_{1}\right)$ and defining $A:=A_{1} \cup A^{\prime}, B:=B_{1} \cup B^{\prime}$. In this case the partial type $r(x)$ will still imply $q(x)$ but the reverse need not hold.

Remark 4.9. By symmetry of $\mathfrak{m}$, the analogue of 4.8 is true for types $p(y)$ such that $p(y) \vdash \mathcal{P}(y)$, and since $\mathcal{Q}, \mathcal{P}$ partition $M$, this covers all 1-types, which are sufficient for saturation.

\section{Ultrapowers OF MODElS OF $T_{\mathfrak{m}}$}

Convention 5.1. In this section, $\mathfrak{m}$ is an arbitrary but fixed parameter.

By 4.8 to ensure a model of $T_{\mathfrak{m}}$ with large leaves is $\lambda^{+}$-saturated, it suffices to realize $R$-types. In our main proofs, we focus on saturating regular ultrapowers. This section gives some basic additional features of the ultrapower case.

Fact 5.2. Suppose $I$ is an infinite set and $\mathcal{D}$ is a regular ultrafilter on $I,|I|=\lambda$. Then for any infinite model $M$ in a countable language, including but not limited to models of $T_{\mathfrak{m}}$, the ultrapower $N=M^{I} / \mathcal{D}$ is $\aleph_{1}$-saturated.

Proof. See for example Chang and Keisler [2] 6.1.1.

Fact 5.3 (see [14]). Saturation of regular ultrapowers reduces to saturation of $\varphi$ types, that is, if $M$ is a model in a countable language and $\mathcal{D}$ is a regular ultrafilter 
on $\lambda$, then $M^{\lambda} / \mathcal{D}$ is $\lambda^{+}$-saturated if and only if it is $\lambda^{+}$-saturated for $\varphi$-types, for every formula $\varphi$.

Recall from Convention 1.14 that a regular ultrafilter $\mathcal{D}$ on a set $I,|I|=\lambda$ is called "good for" a countable theory $T$ if for some, equivalently every, $M \models T$, the ultrapower $M^{I} / \mathcal{D}$ is $\lambda^{+}$-saturated. (The equivalence is by regularity, see [9. 2.1a.)

Fact 5.4. Suppose $I$ is an infinite set and $\mathcal{D}$ is a regular ultrafilter on $I,|I|=\lambda$. Then for any infinite model $M$ in a countable language, including but not limited to models of $T_{\mathfrak{m}}$ :

(1) Suppose in addition that $\mathcal{D}$ is good for every countable stable theory. Then any infinite definable subset of $N=M^{I} / \mathcal{D}$, and indeed any infinite internal predicate in $N$, has size at least $\lambda^{+}$.

(2) Suppose in addition $\mathcal{D}$ is good for the theory of the random graph. Then:

(a) for any two disjoint $A, B \subseteq M^{I} / \mathcal{D}$, there is an internal predicate separating $A$ and $B$.

(b) $\mathcal{D}$ is good for every countable stable $T$.

(c) given any countable sequence $\left\langle X_{n}: n<\omega\right\rangle$ of definable sets and any $A \subseteq N$ of size $\leq \lambda$ such that $X_{n} \supsetneq X_{n+1} \supseteq A$ for all $n<\omega$, there is an internal predicate $X_{\infty}$ such that $X_{n} \supsetneq X_{\infty} \supsetneq A$ for all $n<\omega$. Thus, if $A$ is infinite, the intersection $\bigcap_{n} X_{n}$ has size $\geq \lambda^{+}$in $N$.

Proof. (1) By [33] Theorem 5.1(1)-(2) p. 379, the minimum product of an unbounded sequence of finite or infinite cardinals modulo $\mathcal{D}$ must be at least $\kappa^{+}$ in order to have that for any model $M$ of any countable stable theory, $M^{I} / \mathcal{D}$ is $\kappa^{+}$-saturated. (In fact, this condition is both necessary and sufficient.)

(2)(a) In fact, this characterizes $\mathcal{D}$ being good for the theory of the random graph, see [17] $\S 3$ p. 1585.

(2)(b) The stable theories are below the unstable theories in Keisler's order. See 33 Theorem 4.8. p. 379, which says that any ultrafilter which is good for some unstable theory must have so-called $\operatorname{lcf}(\omega, \mathcal{D}) \geq \lambda^{+}$, 33. Definition 3.5 p. 357 . Thus, $\mu(\mathcal{D}) \geq \lambda^{+}$(this is the quantity mentioned above, informally, the product of any unbounded sequence of finite cardinals modulo $\mathcal{D}$; it is the $\mu$ defined in 33 ] Theorem 3.12 p. 357). It follows from the last line of the proof of (1) that the ultrafilter is good for any countable stable theory.

(2)(c) See 14] Lemma 9 p. 223. This is a consequence of the a priori weaker fact that $\operatorname{lcf}(\omega, \mathcal{D}) \geq \lambda^{+}$. The last sentence then follows from (1) applied to $X_{\infty}$.

Corollary 5.5. If $M_{0} \models T_{\mathfrak{m}}, \mathcal{D}$ is a regular ultrafilter on $\lambda$ which is good for the random graph, and $M=\left(M_{0}\right)^{\lambda} / \mathcal{D}$, then $M$ is weakly $\lambda^{+}$-saturated in the sense of 4.7. In particular, the hypotheses of 4.8 hold for $M$.

Proof. Following the notation of 4.7, for (1), fix $\eta \in \lim \left(\mathcal{T}_{1}\right)$. By Fact [5.2, $M$ is $\aleph_{1}$-saturated, so we can choose $A \subseteq M$ which is countably infinite and which has the property that $Q_{\eta \uparrow n}^{M}(a)$ for all $a \in A$ and $n<\omega$. Apply 5.4(2)(c) using $Q_{\eta\lceil n}^{M}$ for $X_{n}$ and this $A$, and let $X_{\infty}$ be as given there. Then $X_{\infty}$ is contained in the "leaf" we are studying, and it is an infinite internal predicate, so by 5.4(1) it has size at least $\lambda^{+}$, so the leaf does as well. The parallel fact for $P$ is proved symmetrically.

For $4.7(2)$, let $\rho \in \lim \left(\mathcal{T}_{1}\right)$ be the leaf of $c$. It follows from the axioms, see e.g. Definition 2.4 or Observation 4.3 that we can choose some $\eta \in \lim \left(\mathcal{S}_{\rho}\right)$ (since this set has size continuum, so in particular is nonempty). Then $R$ behaves as a 
bipartite random graph between $Q_{\{\rho\}}^{\infty}[M]$ and $P_{\{\eta\}}^{\infty}[M]$, so as $b$ belongs to the first of these sets, it follows by $\aleph_{1}$-saturation (Fact [5.2) that $\left\{b:(c, b) \in R^{M}\right\}$ is an infinite definable set. Thus by 5.4(1) this set has size at least $\lambda^{+}$.

Conclusion 5.6 gives a sufficient collection of types to realize in order to saturate regular ultrapowers for self-dual $\mathfrak{m}$ (our main case following 6.16] below).

Conclusion 5.6. Suppose $I$ is an infinite set, $|I|=\lambda$, and $\mathcal{D}$ is a regular ultrafilter on I which is good for the theory of the random graph. Suppose that $\mathfrak{m}=\operatorname{dual}(\mathfrak{m})$. Let $M_{0} \models T_{\mathfrak{m}}$. Then in order to show that $M=\left(M_{0}\right)^{I} / \mathcal{D}$ is $\lambda^{+}$-saturated, it is sufficient to show that:

$(\star)_{M_{0}, I, \mathcal{D}}$ every partial type of $M$ of the form

$$
r(x)=\left\{Q_{\nu}(x)\right\} \cup\{R(x, a): a \in A\}
$$

is realized, where $\nu \in \mathcal{T}_{1, n}$ for some $n<\omega, A \subseteq M$ and $|A| \leq \lambda$.

Proof. Case 1. For types including $\mathcal{Q}(x)$, by 5.4(2) and our present assumption, the conclusion of 5.4(2)(b) holds. Hence by 5.5, second sentence, the hypothesis of 4.8 holds. Hence, by Claim 4.8, it suffices to deal with partial types of the form

$$
r(x)=\left\{Q_{\rho\lceil n}(x): n<\omega\right\} \cup\{R(x, a): a \in A\} \cup\{\neg R(x, b): b \in B\}
$$

where $\rho \in \lim \left(\mathcal{T}_{1}\right)$ and for some $W \subseteq \lim \left(\mathcal{S}_{\rho}\right)$, we have $A \subseteq P_{W}^{\infty}[M]$ with $|A|=\lambda$, and $B \in P^{M} \backslash A$ with $|B|=\lambda$.

As saturation of regular ultrapowers reduces to saturation of $\varphi$-types, it suffices to consider $\nu \in \mathcal{T}_{1, n}$ for some $n<\omega$, and so to deal with a partial type of the form

$$
r(x)=\left\{Q_{\nu}(x)\right\} \cup\{R(x, a): a \in A\} \cup\{\neg R(x, b): b \in B\} .
$$

Note that the assertion that $r(x)$ is a partial type means that for some $\rho^{\prime}$ with $\nu \unlhd \rho \in \lim \left(\mathcal{T}_{1}\right)$ and some $W \subseteq \lim \left(\mathcal{S}_{\rho^{\prime}}\right)$, we have $A \subseteq P_{W}^{\infty}[M]$ with $|A|=\lambda$, and $B \in P^{M} \backslash A$ with $|B|=\lambda$.

As we are assuming $\mathcal{D}$ is good for the theory of the random graph, by 5.4(2) we can assume there is an internal predicate $X$ separating $A$ and $B$, so let us justify that it sufficies to realize the positive part of the type. Enumerate $r$ as $\left\langle R\left(x, a_{\alpha}\right)\right.$ : $\beta<\lambda, \alpha=2 \beta\rangle$ and $\left\langle\neg R\left(x, b_{\alpha}\right): \beta<\lambda, \alpha=2 \beta+1\right\rangle$. Let $\left\{X_{\alpha}: \alpha<\lambda\right\} \subseteq \mathcal{D}$ be a regularizing family. Let $f:[\lambda]^{<\aleph_{0}} \rightarrow \mathcal{D}$ be the "distribution" given by sending $\sigma$ to

$$
\left\{t \in I: M \models(\exists x)\left(Q_{\nu}(x) \wedge \bigwedge_{\alpha \in \sigma \text { even }} R\left(x, a_{\alpha}[t]\right) \wedge \bigwedge_{\alpha \in \sigma \text { odd }} \neg R\left(x, b_{\alpha}[t]\right)\right)\right\} \cap \bigcap_{\alpha \in \sigma} X_{\alpha} .
$$

Then it is straightforward to see that $r$ is realized if and only if $f$ has a multiplicative refinement. Let $g$ be the refinement of $f$ given by

$$
\sigma \mapsto f(\sigma) \cap\left\{t \in I: M \models \bigwedge_{\alpha \in \sigma \text { even }} X\left(a_{\alpha}[t]\right) \wedge \bigwedge_{\alpha \in \sigma \text { odd }} \neg\left(X\left(b_{\alpha}[t]\right)\right)\right\} .
$$

Now let us verify: $g$ is a function with domain $[\lambda]^{<\aleph_{0}}$ (trivial), $\operatorname{range}(g) \subseteq \mathcal{P}(\lambda)$ (trivial), range $(g) \subseteq \mathcal{D}$ (by the choice of $X), g$ is multiplicative (by its definition), and $g$ refines $f$ (by choice of $X$ and the properties of the random graph).

Case 2. For types including $P(x)$, we use the fact that $\mathfrak{m}$ is self-dual, so for any type on the left, there is a type on the right with an identical distribution. One will have a multiplicative refinement (i.e. be realized) if and only if the other does.

As $M \models(\forall x)(\mathcal{P}(x) \vee \mathcal{Q}(x))$, this finishes the proof that $M$ is $\lambda^{+}$-saturated. 
Remark 5.7. Assume $\operatorname{dual}(\mathfrak{m})=\mathfrak{m}$, we have that $\operatorname{dual}\left(M_{0}\right)$, defined naturally, is a model of $T_{\mathfrak{m}}$. If $\operatorname{dual}\left(M_{0}\right)$ is isomorphic to $M_{0}$, then the ultrapowers of the two models are isomorphic, and so they have the same saturation. But maybe $\operatorname{dual}\left(M_{0}\right) \neq M_{0}$. However we know that $T h\left(M_{0}\right)=T h\left(\operatorname{dual}\left(M_{0}\right)\right)$ hence it is well known that $\left(M_{0}\right)^{\lambda} / \mathcal{D},\left(\operatorname{dual}\left(M_{0}\right)\right)^{\lambda} / \mathcal{D}$ are $L_{\infty, \lambda^{+}}$-equivalent, hence the argument above holds. (Really what we need is just Keisler's lemma that if $M_{1} \equiv M_{2}$ in a language of size no more than $\lambda$ and $\mathcal{D}$ is a regular ultrafilter on $\lambda$, then $\left(M_{1}\right)^{\lambda} / \mathcal{D}$ is $\lambda^{+}$-saturated if and only if $\left(M_{2}\right)^{\lambda} / \mathcal{D}$ is $\lambda^{+}$-saturated.)

Discussion 5.8. If we were not assuming $\mathfrak{m}=\operatorname{dual}(\mathfrak{m})$, then we would just need to add the parallel for types containing $\mathcal{P}(x)$. We may do this in at least two ways: either we update $(\star)_{M_{0}, I, \mathcal{D}}$ to include the parallel condition for $\mathcal{P}(x)$ replacing $\mathcal{Q}(x)$ (with the corresponding minor notational changes), or else, we add the condition $(\star)_{\operatorname{dual}\left(M_{0}\right), I, \mathcal{D}}$, where $\operatorname{dual}\left(M_{0}\right)$ is a model of $T_{\mathrm{dual}(\mathfrak{m})}$, since $\mathcal{Q}$ in $\operatorname{dual}\left(M_{0}\right)$ is the parallel of $\mathcal{P}$ in $M_{0}$.

\section{SIZES}

In $\$ 2$ we built theories $T_{\mathfrak{m}}$ from templates $\mathfrak{m}$ under quite general conditions on the template edge relation $\mathcal{R}$. It was mentioned that for our main work below, we will want to choose our $\mathcal{R}$ 's carefully to reflect certain sequences of sparse random graphs. The construction of these sparse random graphs is the task of this section, leading to the definition of our continuum many parameters in 6.22 (building on 6.12). Claim 6.17 verifies any such parameter satisfies the axioms of $\$ 2$

Some remarks on the problems this section must solve and how it solves them:

First of all, 6.22 must be able to output continuum many parameters which have a reasonable chance of being "very different" from each other, with no one of any pair obviously more or less complex than the other; more on this soon.

Second, the data of a parameter is essentially 12 summarized by the data of a bipartite graph associated to every pair $(\nu, \eta) \in \mathcal{R}_{k}$ for every $k<\omega$ (i.e., the bipartite graph corresponding to the pattern of $\mathcal{R}_{k+1}$-edges on their immediate successors). For the parameters built here, this associated graph will be an invariant of the level $k$ (i.e., branching at each level $k$ is uniform, we specify for each level $k$ a bipartite graph of the right size, and then for every $\mathcal{R}_{k}$-edge $(\nu, \eta)$ the pattern of $\mathcal{R}_{k+1}$-edges between the immediate successors of $\nu$ and $\eta$ copies this fixed bipartite graph). So we may think of our theories as reflecting bipartite graph sequences.

In considering how to construct and compare bipartite graph sequences, remember that the resulting $\mathfrak{m}$ should be self-dual. Random bipartite graphs may not have the needed symmetry. So we shall simply construct sequences of graphs, allowing self-loops (edges from a vertex to itself), and only at the last minute convert them to symmetric bipartite graphs by doubling the vertices (6.8).

The key point which will illuminate comparison is that we require our sequences of graphs $\left\langle E_{i}: i\langle\omega\rangle\right.$ to have a well-defined notion of large and small in the following sense. The $i$-th graph will have vertex set $m_{i}=\left\{0, \ldots, m_{i}-1\right\}$, and "small" and "large" will depend on $i$. We shall require that for every small subset $u$ of $m_{i}$, there is some vertex connected to all elements of $u$, whereas for every large subset $v$, there is no vertex connected to all elements of $v$. Briefly, all small subsets

\footnotetext{
${ }^{12}$ Set aside the level function for the moment.
} 
are covered and no large subsets are. A main aim of the section is showing this can be arranged for certain explicitly given growth rates by using fairly sparse random graphs. (A clear picture of these rates is essential as they will interface directly with the chain condition in 8.2 , in a non-obvious interaction of finite and infinite 13 )

Finally, to motivate incomparability, one may imagine that the "small" and "large" conditions could transmute in later sections into conditions on consistency of sets of formulas, and so hope to build sequences of graphs with orthogonal notions of small and large, meaning perhaps there would be many indices $i$ for which "small" in the first sequence is larger than "large" in the second sequence, and vice versa.

Our solution is to start with a single sequence of graphs $\left\langle E_{i}: i\langle\omega\rangle\right.$ and a family of level functions which is independent in the sense of [5] (see 6.20). The correct variation in large and small is naturally produced by "turning off and on" the constraints of $E_{i}$ in accordance with a level function; this is very robust, as summarized in 6.22. (This idea seems to have a certain naturalness; had we not introduced level functions in $\$ 2$, at this point we might be obliged to define them.)

We begin by laying out requirements on the sequence of integers which will specify the branching (and so the sizes of the vertex sets in our sequence of graphs).

Definition 6.1. Say that the countable sequence $\bar{m}=\left\langle m_{i}: i<\omega\right\rangle \in{ }^{\omega}(\omega \backslash\{0\})$ is $a$ fast sequence when for each $i, m_{i}^{\circ}:=\prod_{j<i} m_{i}$ satisfies

$$
m_{i} \geq\left(\left(m_{i}^{\circ}\right)^{i^{i}}\right)^{4\left(m_{i}^{\circ}\right)^{i^{i}}} .
$$

Since the $m_{i}^{\circ}$ 's are uniquely determined by the $m_{i}$ 's, we may sometimes display $\bar{m}$ as a sequence of pairs $\left\langle\left(m_{i}, m_{i}^{\circ}\right): i<\omega\right\rangle$, while referring to it as a sequence of singletons. To avoid triviality, we assume $m_{0}>1$.

In the next definition, note the $E_{i}$ are graphs, not bipartite graphs. We have chosen to allow self-loops (i.e. $(a, a)$ can be an edge), but this is not crucial.

Definition 6.2. Let $\bar{m}$ be a fast sequence.

(1) $\bar{E}$ will denote a sequence of graphs for $\bar{m}$ when each $E_{i} \subseteq\left[m_{i}\right]^{2} \cup\{(a, a)$ : $\left.a \in\left[m_{i}\right]\right\}$ and for $i=0$ we have equality.

(2) $\bar{E}$ is a good sequence of graphs for $\bar{m}$, or just good for $\bar{m}$, when in addition, there is some finite $i_{*}$ such that for all $i \geq i_{*}$,

(i) if $u \subseteq m_{i}$,

$$
|u| \leq\left(m_{i}^{\circ}\right)^{i^{i}}
$$

then $u$ is " $\bar{E}$-small," meaning that that there is s such that

$$
(\forall t \in u)\left(E_{i}(s, t)\right) .
$$

(ii) if $u \subseteq m_{i}$ and

$$
|u| \geq \frac{m_{i}}{\left(m_{i}^{\circ}\right)^{i}}
$$

then $u$ is "E-large," meaning that there is no s such that

$$
(\forall t \in u)\left(E_{i}(s, t)\right) .
$$

We shall omit $\bar{E}$ in "small" and "large" when it is clear from the context.

\footnotetext{
13 and of genericity and randomness in the finite and in the infinite settings.
} 
The definition of "small" is used in the proof of existence of a model completion and in the proof of 10.22 below, and the definition of "large" in the proof of nonsaturation below.

Convention 6.3. For the next few lemmas, let $g: \mathbb{N} \rightarrow \mathbb{N}$ be the function given by

$$
g(i)=2\left(m_{i}^{\circ}\right)^{i^{i}} .
$$

(of course, we could have called this $g\left(m_{i}\right)$.)

Fact 6.4 (see e.g. Bollobás [1] Corollary 3.14). Let $G$ be a graph. Let $\Delta(G)$ denote the maximal degree of $G$ and let $G_{p}$ denote a graph from $\mathcal{G}_{n, p}$, random graphs on $n$ vertices with edge probability $p=p(n)$. If $p n / \log n \rightarrow \infty$, then a.e. $G_{p}$ satisfies

$$
\Delta\left(G_{p}\right)=\{1+o(1)\} p n .
$$

In 6.4, note that $p$ is a function of $n$.

Observation 6.5. Let $g$ be from 6.3 and suppose

$$
p=p\left(m_{i}\right)=\frac{1}{\left(m_{i}\right)^{\frac{1}{g(i)}}} .
$$

Then $p \cdot m_{i} / \log m_{i} \rightarrow \infty$, so 6.4 applies. In particular, as $i \rightarrow \infty$, the proportion of $G \in \mathcal{G}_{m_{i}, p}$ which have no vertices of "large" degree goes to 1 .

Proof. Recalling that $g(i)$ from 6.3 is monotonic and strictly increasing and approaches $\infty$, and recalling that $p=\frac{1}{\left(m_{i}\right)^{\frac{1}{g(i)}}}$ we have

$$
\lim _{i \rightarrow \infty}\left(\frac{1}{\left(m_{i}\right)^{\frac{1}{g(i)}}}\right)\left(\frac{m_{i}}{\log m_{i}}\right)=\lim _{i \rightarrow \infty}\left(\frac{m_{i}^{1-\frac{1}{g(i)}}}{\log m_{i}}\right)=\infty .
$$

So Fact 6.4 applies (actually $g(i) \geq 2$ suffices) and for some fixed constant $c$, in almost every $G_{p, m_{i}}$, every vertex has degree $\leq c p m_{i}$. Let us verify that this degree is eventually not "large" in the sense of 6.2 . For this it would suffice to show that

$$
\frac{m_{i}}{\left(m_{i}^{\circ}\right)^{i^{i}}} \text { is quite a bit bigger than } \frac{m_{i}}{m_{i}{ }^{1 / g(i)}}
$$

[the left-hand side is the threshold for "large" from 6.2 and the right-hand side is $\left.p \cdot m_{i}\right]$ and for this it would suffice to show that

$$
\left(m_{i}^{\circ}\right)^{i^{i}} \text { is quite a bit smaller than } m_{i}{ }^{1 / g(i)} \text {. }
$$

which is ensured by (a) of Definition 6.1 and the definition of $g$ in 6.3 .

Observation 6.6. For each $i,\left(m_{i}^{\circ}\right)^{i^{i}}<\left(m_{i}\right)^{1 / 4}$.

Proof. We verify that

$$
\left(m_{i}^{\circ}\right)^{i^{i}}<\left(\left(\left(m_{i}^{\circ}\right)^{i^{i}}\right)^{4\left(m_{i}^{\circ}\right)^{i^{i}}}\right)^{1 / 4} \leq\left(m_{i}\right)^{1 / 4}
$$

recalling 6.1 (a) .

Lemma 6.7. Let $E_{i} \subseteq\left[m_{i}\right]^{2} \cup\left\{(a, a): a \in\left[m_{i}\right]\right\}$ be symmetric and random with probability $p$ from 6.5 for each pair to be an edge. In such a graph, with probability close to 1 (for us nonzero probability is sufficient): 
(1) for every $u \subseteq m_{i}$, if

$$
|u| \leq\left(m_{i}^{\circ}\right)^{i^{i}}
$$

then there is $s$ so that $(\forall t \in u)\left(E_{i}(s, t)\right)$.

(2) for every $u \subseteq m_{i}$, if

$$
|u| \geq \frac{m_{i}}{\left(m_{i}^{\circ}\right)^{i}}
$$

then there does not exist $s$ so that $(\forall t \in u)\left(E_{i}(s, t)\right)$.

Remark 6.8. For now we will define a graph on $m_{i}$ vertices with edge relation $E_{i}$, allowing self loops. In the translation from $E_{i}$ to $\mathcal{R}_{i}$ in 6.12 below, we will use this graph to make a bipartite graph, which will then be symmetric [in the sense that the derived $\mathfrak{m}=\operatorname{dual}(\mathfrak{m})]$ by construction.

Proof. Recall $[n]$ denotes the $n$-element set $\{0, \ldots, n-1\}$. We define a probability measure $\mu=\mu_{i}$ on $\left\{X: X \subseteq\left[m_{i}\right]^{2} \cup\left\{(a, a): a \in\left[m_{i}\right]\right\}\right\}$ by flipping a coin $c_{\{a, b\}}$ for each potential edget14 with probability of heads (=yes) being

$$
\frac{1}{\left(m_{i}\right)^{\frac{1}{g(i)}}}
$$

where $g(i)$ is again from 6.3. Condition (2) will be handled by Observation 6.5, so let us address condition (1). Let us say that a set $v \subseteq\left[m_{i}\right]$ is "covered" if there exists $b \in\left[m_{i}\right]$ such that $(\forall t \in v)\left(E_{i}(b, t)\right)$. Clearly it will suffice to show that all sets of size $\left(m_{i}^{\circ}\right)^{i^{i}}$ are covered.

Let $\mathcal{E}_{i}^{1}$ be the probability that an arbitrary (given) $v \subseteq\left[m_{i}\right]$ of size $x$ is not covered ( $x$ to be chosen later, in our main case $\left(m_{i}^{\circ}\right)^{i^{i}}$.) Before bounding $\mathcal{E}_{i}^{1}$, note that the probability that some $v \subseteq\left[m_{i}\right]$ of size $x$ is not covered is

$$
\left(\begin{array}{c}
m_{i} \\
x
\end{array}\right)\left(\mathcal{E}_{i}^{1}\right)
$$

i.e. the number of ways to choose a $v$ of size $x$ times the probability that a given $v$ is not covered. Now

$$
\mathcal{E}_{i}^{1}=\left(1-\frac{1}{\left(m_{i}\right)^{x / g(i)}}\right)^{m_{i}}
$$

[the term in parentheses represents the chance that each particular $b$ fails to cover $v$; there are $m_{i}$ choices for $\left.b\right]$. Recalling that $(1-1 / n)^{n}$ is well approximated by $\frac{1}{e}$ for large $n$, we may rewrite the right side of (h) as

$$
\left(\left(1-\frac{1}{\left(m_{i}\right)^{x / g(i)}}\right)^{\left(m_{i}\right)^{x / g(i)}}\right)^{\frac{m_{i}}{m_{i} x / g(i)}}
$$

and then (ii) is well approximated by

$$
\frac{1}{e^{\left[\left(m_{i}\right)^{1-x / g(i)}\right]}} .
$$

We need equation (ii) to be very small, so we need $e^{\left[\left(m_{i}\right)^{1-x / g(i)}\right]}$ to be very large, so we need $\left[\left(m_{i}\right)^{1-x / g(i)}\right]$ to be very large, so we need $1-x / g(i)$ to be not too small. For our present calculations, let us verify that it suffices to have $x / g(i)=1 / 2$,

\footnotetext{
14 note that this edge relation is by definition symmetric.
} 
which is satisfied in our main case when $x=\left(m_{i}^{\circ}\right)^{i^{i}}$ from 6.2 and $g(i)$ is from 6.3 In this case, $\mathcal{E}_{i}^{1}$ is well approximated by

$$
\frac{1}{e^{\sqrt{m_{i}}}}
$$

hence an upper bound for equation (g) is well approximated by

$$
\left(m_{i}\right)^{x}\left(\mathcal{E}_{i}^{1}\right) \approx\left(e^{x \ln m_{i}}\right)\left(\frac{1}{e^{\sqrt{m_{i}}}}\right)=\frac{1}{e^{\sqrt{m_{i}}-x \ln m_{i}}} .
$$

It is sufficient for us that the exponent be nonnegative and growing; for instance, $x<\left(m_{i}\right)^{1 / 4}$ suffices for us, and is indeed satisfied in our main case $x=\left(m_{i}^{\circ}\right)^{i^{i}}$ recalling [6.6] This completes the proof.

Conclusion 6.9. If $\bar{m}$ is a fast sequence, there exists $\bar{E}$ which is good for $\bar{m}$.

Remark 6.10. The bounds in 6.2 are for definiteness, what we really use is 6.76.9: $\bar{m}$ grows quickly enough to find a sequence of graphs $\bar{E}$ with a coherent and growing notion of "small" and "large" (every small set has an $x$ connected to all of its elements, and no large set has an $x$ connected to all of its elements). Zero-one laws [34] suggest much flexibility in choosing such bounds.

Next, given the three key ingredients $\bar{m}, \bar{E}$, and a level function $\xi$ (recall 2.2), we construct a parameter $\mathfrak{m}$ whose sequence of reduced graphs naturally reflects $\bar{E}$.

Notation 6.11. For $\bar{m}$ a countable sequence of natural numbers and $\eta \in{ }^{\omega>} \omega$ or $\eta \in{ }^{\omega} \omega$, the notation $\eta<\bar{m}$ means $\eta(i)<m_{i}$ for all $i<\operatorname{lgn}(\eta)$ (i.e. in $\operatorname{dom}(\eta)$ ).

Definition 6.12 (The parameters we use). For any fast sequence $\bar{m}=\left\langle m_{i}: i<\omega\right\rangle$, any $\bar{E}=\left\langle E_{i}: i<\omega\right\rangle$ which is good for $\bar{m}$, and any level function $\xi \in{ }^{\omega} 2$, we define a parameter $\mathfrak{m}=\operatorname{par}[\bar{m}, \bar{E}, \xi]$ as follows:

(1) if $\ell=1,2, \mathcal{T}_{\mathfrak{m}, \ell}=\left\{\eta: \eta \in{ }^{\omega>} \omega, \eta<\bar{m}\right\}$.

In particular, for each $\nu \in \mathcal{T}_{\ell}$, if $\nu \in \mathcal{T}_{\ell, i}$ then $\left|\operatorname{ims}_{\mathcal{T}_{\ell}}(\nu)\right|=m_{i}$.

(2) for the next two items, for $i<\omega$, let $E_{i}^{1}=E_{i}$, and let $E_{i}^{0}$ be $m_{i} \times m_{i}$, the complete graph with self-loops.

(3) for $i=0, \mathcal{R}_{0}=\{(\emptyset, \emptyset)\}$.

(4) for $i+1$,

$$
\mathcal{R}_{i+1}=\left\{\left(\eta_{1}, \eta_{2}\right): \eta_{\ell} \in \mathcal{T}_{\ell, i+1}, \quad\left(\eta_{1}\left\lceil i, \eta_{2}\lceil i) \in \mathcal{R}_{i}, \quad\left(\eta_{1}(i), \eta_{2}(i)\right) \in E_{i}^{\xi(i)}\right\} .\right.\right.
$$

Convention 6.13. In what follows, we use the letters $\mathfrak{m}$ and $\mathfrak{n}$, often with subscripts, for parameters. We will often write e.g.

$$
\mathfrak{m}=\mathfrak{m}[\bar{m}, \bar{E}, \xi] \text { or } \mathfrak{n}=\mathfrak{n}[\bar{m}, \bar{E}, \xi] \text { or } \mathfrak{m}=\operatorname{par}[\bar{m}, \bar{E}, \xi]
$$

to indicate the dependence of a given parameter on the three inputs.

Observation 6.14. Suppose $\bar{m}, \bar{E}$ are as above and $\xi_{1}, \xi_{2} \in{ }^{\omega}\{0,1\}$ and $\mathfrak{m}_{\ell}=$ $\operatorname{par}\left[\bar{m}, \bar{E}, \xi_{\ell}\right]$. If we have $\xi_{2}^{-1}\{1\} \subseteq \xi_{1}^{-1}\{1\}$, then $\mathcal{R}^{\mathfrak{m}_{1}} \subseteq \mathcal{R}^{\mathfrak{m}_{2}}$.

(Observation 6.14 will be used in $\$ 12$ below. Informally, if there are more active levels, there are more constraints, so $\mathcal{R}$ has fewer edges.)

Discussion 6.15 (Indexing). So that the key points of the construction in 6.12 are not hidden in the indexing, we review:

(a) $\mathcal{T}_{1,0}=\mathcal{T}_{2,0}=\{\emptyset\}$, and $\mathcal{R}_{0}=\{(\emptyset, \emptyset)\}$. 
(b) For $i+1$, recall that the elements of $\mathcal{T}_{\ell, i+1}$ are sequences of length $i+1$, i.e. functions $\eta$ from $\{0, \ldots, i\}$ to $\omega$ subject to the constraint that $\eta(j)<m_{j}$ for each $j \leq i$.

(c) $\mathcal{T}_{\ell, 1}$ has $m_{0}$ nodes; in general, for $k>0 \mathcal{T}_{\ell, k}$ has $\prod_{j<k} m_{j}=m_{k}^{\circ}$ nodes. Also for $k=0, m_{k}^{\circ}=1=\prod_{j<0} m_{j}$.

(d) If $\eta \in \mathcal{T}_{\ell, k}$ then $\eta$ has $m_{k}$ immediate successors in $\mathcal{T}_{\ell, k+1}$, so it follows that $\mathcal{T}_{\ell, k+1}$ has size $\left(\prod_{j<k} m_{j}\right) \cdot m_{k}=\prod_{j<k+1} m_{j}=m_{k+1}^{\circ}$, as desired.

(e) If $(\eta, \nu) \in \mathcal{T}_{1, k} \times \mathcal{T}_{2, k}$ and $(\eta, \nu) \in \mathcal{R}_{k}$, then letting $A=\operatorname{ims}_{\mathcal{T}_{1, k+1}}(\eta)$, $B=\operatorname{ims}_{\mathcal{T}_{2, k+1}}(\nu)$, if $k+1$ is an active level, then $\left(A, B, \mathcal{R}_{k+1} \uparrow A \times B\right)$ is isomorphic as a bipartite graph to $\left(m_{k}, m_{k}, E_{k}\right)$. If $k+1$ is a lazy level, it is a complete bipartite graph.

(f) In a slogan: given two elements of length $k$ connected by $\mathcal{R}_{k}, E_{k}$ gives us the pattern of edges between their sets of successors, provided those successors are at an active level. Note to the reader: so there is no confusion, we repeat that $\xi(k)$ affects edges at level $k+1$, recalling 2.5 .

Note that 6.12 establishes self-duality essentially for free. (The key point is that each of the $\mathcal{R}_{i}$ 's is symmetric as a bipartite graph; from each $\mathcal{R}_{i+1}$ one can naturally recover $E_{i}^{\xi(i+1)}$ by identifying each vertex on the left with its parallel on the right.)

Claim 6.16. If $\mathfrak{m}$ is constructed from any $\bar{m}, \bar{E}$ which is good for $\bar{m}$ as in 6.12 , and $\xi$ which satisfies $\xi(i)=0$ for $i<i_{*}=i_{*}(\bar{E})$ (recalling $6.2(2)$ ), then $\mathfrak{m}=\operatorname{dual}(\mathfrak{m})$.

Proof. Immediate: this is the symmetry of the construction in 6.12,

Claim 6.17. If $\mathfrak{m}$ is constructed from any $\bar{m}, \bar{E}$, $\xi$ as in 6.16, then $\mathfrak{m}$ is a parameter. Moreover, for every $k<\omega$,

(1) if $\left\{\rho_{i}: i<s\right\} \subseteq \mathcal{T}_{2, k+1}$ and $s \leq\left(m_{k}^{\circ}\right)^{k^{k}}$, and $\nu \in \mathcal{T}_{1, k}$ with $\left(\nu, \rho_{i}\lceil k) \in \mathcal{R}_{k}\right.$ for each $i<s$, then there exists an immediate successor $\nu^{\frown}\langle t\rangle$ of $\nu$ such that $\left(\nu^{\curlyvee}\langle t\rangle, \rho_{i}\right) \in \mathcal{R}_{k+1}$ for each $i<s$.

(2) the parallel condition to (1) holds switching $\mathcal{T}_{2, k+1}$ and $\mathcal{T}_{1, k+1}$.

Proof. We check 2.3 and 2.6, 2.3(1), (2), (3) are obvious from our construction. (4) holds since the degree of a vertex in $E_{i}^{a}$ is at least two. (5) is immediate from the construction and (6) does not require verification.

For 2.6. it will be helpful to first prove the "moreover" clauses of our Claim, which greatly strengthen Extension in one aspect. Note that as $\mathfrak{m}=\operatorname{dual}(\mathfrak{m})$ it suffices to prove (1). Consider $s \leq\left(m_{k}^{\circ}\right)^{k^{k}}$ and $\nu \in \mathcal{T}_{1, k}$ and $\rho_{0}, \ldots \rho_{s-1} \in \mathcal{T}_{2, k+1}$ such that $\left(\nu, \rho_{i}\lceil k) \in \mathcal{R}_{k}\right.$ for $i<s$. Write $\rho_{i}=\left(\rho_{i}\lceil k)^{\wedge}\left\langle\ell_{i}\right\rangle\right.$ for each $i<s$. In the graph $E_{k},\left\{\ell_{i}: i<s\right\}$ is a small subset of $\left[m_{k}\right]$, so there is some $t \in\left[m_{i}\right]$ such that $\left(t, \ell_{i}\right)$ is an edge in $E_{k}$ for every $i<s$. Recall that if $\xi(k)=1$, for each $i<s$, to form $\mathcal{R}_{k}$ in 6.12 we simply put a bipartite copy of $E_{k}$ [always the same $E_{k}$ ] between the immediate successors of $\nu$ and the immediate successors of $\rho_{i}\lceil k$. As a result, for each $i<s$ (we just look one by one), $\left(\nu^{\curlyvee}\langle t\rangle, \rho_{i}\left\lceil k^{\curlyvee}\left\langle\ell_{i}\right\rangle\right) \in \mathcal{R}_{k+1}\right.$. In other words, $\nu^{\frown}\langle t\rangle$ connects to each $\rho_{i}$ (again note: even though they may not have an immediate common predecessor). If $\xi(k)=0$, we use a complete graph instead of $E_{i}$, so this is all true a fortiori and there are even many such elements. This proves (1), and also (2).

Finally, we check the extension conditions from 2.6, and as $\mathfrak{m}$ is self-dual, it will suffice to prove one way. Applying the preceding paragraph in the case $s=k$ tells 
us there is at least one successor of $\nu$ connecting to $\rho_{0}, \ldots \rho_{k-1}$. The additional point is to note that there is not just one $t$ but at least $k+1$. When $\xi(k)=0$, as noted, there will be many such successors, actually all. To see that there are many when $\xi(k)=1$, by induction on $\ell \leq k$ choose $t_{\ell} \in\left[m_{k}\right] \backslash\left\{t_{0}, \ldots, t_{\ell-1}\right\}$ such that $\bigwedge_{i<k}\left(\nu^{\frown}\left\langle t_{\ell}\right\rangle, \rho_{i}\right) \in \mathcal{R}_{k+1}$, as follows: note that we could choose $\rho$ such that $j<\ell \Longrightarrow\left(\nu^{\frown}\left\langle t_{j}\right\rangle, \rho\right) \notin \mathcal{R}_{k+1}$, possible as no vertex has large degree, and apply the previous paragraph to the still small set $\left\{\rho_{i}: i<k\right\} \cup\{\rho\}$ to find $\nu^{\frown}\langle s\rangle$ which connects to $\rho_{0}, \ldots, \rho_{k-1}, \rho$, necessarily for $s \neq t_{j}$ for $j<\ell$, so $s$ can serve as $t_{\ell}$. Continuing in this way, we find the $k+1$ elements for the extension axiom.

Remark 6.18. In the proof of 6.17, it is worth observing the following helpful feature of using the same $E_{k}$ across the entire level. Suppose we have $\nu \in \mathcal{T}_{1, k}$, $\eta, \rho \in \mathcal{T}_{2, k}$, and $(\nu, \eta) \in \mathcal{R}_{k},(\nu, \rho) \in \mathcal{R}_{k}$. Suppose $(i, j)$ and $(i, \ell)$ are edges in $E_{k}$, and to avoid triviality, suppose that $\xi(k)=1$. Then of course $\left(\nu^{\frown}\langle i\rangle, \eta^{\frown}\langle j\rangle\right)$ and $\left(\nu^{\frown}\langle i\rangle, \eta^{\frown}\langle\ell\rangle\right)$ are both in $\mathcal{R}_{k+1}$, but also notice $\left(\nu^{\frown}\langle i\rangle, \eta^{\frown}\langle j\rangle\right)$ and $\left(\nu^{\frown}\langle i\rangle, \rho^{\frown}\langle\ell\rangle\right)$ are both in $\mathcal{R}_{k+1}$, simply because we consulted the same $E_{k}$ in both cases.

We may now invoke the level functions to build a family of parameters whose active levels are independent in the following precise sense.

Notation 6.19. Recall that $A \subseteq \subseteq^{*} B$ means that $\{a \in A: a \notin B\}$ is finite.

Fact 6.20. For any $i_{*}<\omega$, there is $\Xi=\left\{\xi_{\alpha}: \alpha<2^{\aleph_{0}}\right\} \subseteq \omega\{0,1\}$ of size continuum such that:

$$
\begin{array}{r}
\text { if } u \subseteq 2^{\aleph_{0}} \text { is finite and } \beta<2^{\aleph_{0}} \text { is not from } u \text {, then } \\
\qquad \xi_{\beta}^{-1}\{1\} \nsubseteq^{*} \bigcup\left\{\xi_{\alpha}^{-1}\{1\}: \alpha \in u\right\}
\end{array}
$$

and moreover $\xi_{\alpha}(i)=0$ for all $i<i_{*}$ and all $\alpha<2^{\aleph_{0}}$.

Proof. We can use e.g. the existence of a family $\mathcal{G}=\left\{g_{\alpha}: \alpha<2^{\aleph_{0}}\right\} \subseteq{ }^{\omega} \omega$ of continuum many independent functions, see [5] or [3] or [33] Appendix, Theorem $1.5(1)$ p. 656. Recall that this means that each $g \in \mathcal{G}$ is a function from $\omega$ to $\omega$ and for any finite $n$, any distinct $\alpha_{0}, \ldots, \alpha_{n-1}<2^{\aleph_{0}}$, and any values $t_{0}, \ldots, t_{n-1}<$ $\omega$ (not necessarily distinct), the set $\left\{i<\omega: g_{\alpha_{0}}(i)=t_{0} \wedge \cdots \wedge g_{\alpha_{n-1}}(i)=\right.$ $\left.t_{n-1}\right\} \neq \emptyset$. In particular (as we can play with setting the values of any finitely many other functions) it follows from the definition of independent that for any distinct $\alpha_{0}, \ldots, \alpha_{n-1}, \beta<2^{\aleph_{0}}$ and any $s_{0}, \ldots, s_{n-1}, t<\omega$, the set

$$
\left\{i<\omega: g_{\alpha_{0}}(i)=s_{0} \wedge \cdots \wedge g_{\alpha_{n-1}}(i)=s_{n-1} \wedge g_{\beta}(i)=t\right\}
$$

is infinite. For each $g_{\alpha}$ in our family $\mathcal{G}$, let $\xi_{\alpha}$ be the function $i \mapsto\left(g_{\alpha}(i) \bmod 2\right)$. It follows that $\left\{\xi_{\alpha}: \alpha<2^{\aleph_{0}}\right\}$ is as desired.

Since changing the first $i$ values for every $g_{\alpha}$ in the family to be zero does not alter the independence, we can ensure the last line for any finite $i_{*}$ given in advance.

Remark 6.21. We could have also demanded that the sets $\xi_{\alpha}^{-1}\{1\}$ are infinite and pairwise almost disjoint.

Corollary 6.22. Thus, for any fast sequence $\bar{m}$, any $\bar{E}$ which is good for $\bar{m}$, and $\Xi=\left\langle\xi_{\alpha}: \alpha<2^{\aleph_{0}}\right\rangle$ from 6.20 with $i_{*}=i_{*}(\bar{E})$, we can define a set

$$
\mathbf{M}_{*}=\left\{\mathfrak{m}_{\alpha}=\operatorname{par}\left[\bar{m}, \bar{E}, \xi_{\alpha}\right]: \alpha<2^{\aleph_{0}}\right\}
$$


with no repetition. For any $\mathcal{M} \subseteq \mathbf{M}_{*}$, define

$$
\mathcal{I}_{\mathcal{M}}=\left\{v \subseteq \omega: \text { for some } \mathfrak{m}_{\alpha_{0}}, \ldots, \mathfrak{m}_{\alpha_{n-1}} \in \mathcal{M}, v \subseteq^{*} \bigcup\left\{\xi_{\alpha_{\ell}}^{-1}\{1\}: \ell<n\right\}\right\} .
$$

Then if $\mathcal{N} \subseteq \mathbf{M}_{*} \backslash \mathcal{M}$, we will have that:

(a) if $\mathfrak{m}_{\alpha} \in \mathcal{M}$, then $\xi_{\alpha}^{-1}\{1\} \in \mathcal{I}_{\mathcal{M}}$.

(b) if $\mathfrak{m}_{\beta} \in \mathcal{N}$, then $\xi_{\beta}^{-1}\{1\} \neq \emptyset \bmod \mathcal{I}_{\mathcal{M}}$.

Remark 6.23. Corollary 6.22 summarizes a natural sense in which any two elements, or indeed any two disjoint subsets, of $\mathbf{M}_{*}$ are orthogonal.

\section{Possibility Patterns AND Ultrapowers}

We will be interested in analyzing types in regular ultrapowers, and the following set-up is especially useful for this. To readers familiar with "separation of variables" in the sense of [20], there is nothing new here; we simply explain that framework and fix some notation.

The first idea is that a regular ultrafilter on $\lambda$ can be "projected" onto any reasonable Boolean algebra (complete, of size $\leq 2^{\lambda}$, with the $\lambda^{+}$-c.c.) and studied there. Let us give the definition, then discuss how it can be used.

Definition 7.1 (Regular ultrafilters built from tuples, from [20] Theorem 6.13). Suppose $\mathcal{D}$ is a regular ultrafilter on $I,|I|=\lambda$. We say that $\mathcal{D}$ is built from $\left(\mathcal{D}_{0}, \mathfrak{B}, \mathcal{D}_{*}, \mathbf{j}\right)$ when:

(1) $\mathcal{D}_{0}$ is a regular, $|I|^{+}$-good filter on $I$

(2) $\mathfrak{B}$ is a Boolean algebra

(3) $\mathcal{D}_{*}$ is an ultrafilter on $\mathfrak{B}$

(4) $\mathbf{j}: \mathcal{P}(I) \rightarrow \mathfrak{B}$ is a surjective homomorphism such that:

(a) $\mathcal{D}_{0}=\mathbf{j}^{-1}\left(\left\{1_{\mathfrak{B}}\right\}\right)$

(b) $\mathcal{D}=\left\{A \subseteq I: \mathbf{j}(A) \in \mathcal{D}_{*}\right\}$.

Since 7.1 is defined with an eye towards Keisler's order, an important feature of this definition is that the problem of realizing types is naturally projected to the Boolean algebra, too, as 7.2 explains 15

Definition 7.2. Continuing in the notation of 7.1, suppose that $\mathcal{D}$ is built from $\left(\mathcal{D}_{0}, \mathfrak{B}, \mathcal{D}_{*}, \mathbf{j}\right)$. Consider a complete countable $T$ and $M \models T$. Suppose $N=M^{\lambda} / \mathcal{D}$ and $p$ is a partial type over $\kappa \leq \lambda$ parameters in $N$, so $p=\left\langle\varphi_{\alpha}\left(x, a_{\alpha}\right): \alpha<\kappa\right\rangle$. We say that $\overline{\mathbf{b}}$ represents the type $p$ when: for each finite $u \subseteq \kappa$, the Loś map $E$ sends $u \mapsto B_{u}$ where

$$
B_{u}:=\left\{t \in I: M \models(\exists x) \bigwedge_{\alpha \in u}\left\{\varphi_{\alpha}\left(x, a_{\alpha}[t]\right)\right\} .\right.
$$

and $\mathbf{b}_{u}=\mathbf{j}\left(B_{u}\right)$. We write $\bar{B}=\left\langle B_{u}: u \in[\kappa]^{<\aleph_{0}}\right\rangle$, and $\overline{\mathbf{b}}=\left\langle\mathbf{b}_{u}: u \in[\kappa]^{<\aleph_{0}}\right\rangle$.

Continuing in the notation of 7.2 , recall that $\left\langle B_{u}: u \in[\lambda]<\kappa_{0}\right\rangle$ of elements of $I$ is called multiplicative if $B_{u} \cap B_{v}=B_{u \cup v}$ for every $u, v \in[\lambda]^{<\aleph_{0}}$. To say that $\bar{B}$ has a multiplicative refinement in $\mathcal{D}$ means that there is $\bar{B}^{\prime}=\left\langle B_{u}^{\prime}: u \in[\lambda]^{<\aleph_{0}}\right\rangle$ such that for each $u, B_{u}^{\prime} \in \mathcal{D}$ and $B_{u}^{\prime} \subseteq B_{u}$, and moreover, $\bar{B}^{\prime}$ is multiplicative.

\footnotetext{
${ }^{15}$ The next definition seems to suggest that in $\varphi(x, y), \ell(y)=1$. This will be our main case in this paper, but it's not a constraint.
} 
Likewise (just a little more briefly) $\overline{\mathbf{b}}$ has a multiplicative refinement in $\mathcal{D}_{*}$ if there exists a sequence $\overline{\mathbf{b}}^{\prime}=\left\langle\mathbf{b}_{u}^{\prime}: u \in[\lambda]^{<\aleph_{0}}\right\rangle$ of elements of $\mathcal{D}_{*}$ such that $\mathbf{b}_{u}^{\prime} \subseteq \mathbf{b}_{u}$ for each $u \in[\lambda]^{<\aleph_{0}}$, and $\mathbf{b}_{u}^{\prime} \cap \mathbf{b}_{v}^{\prime}=\mathbf{b}_{u \cup v}^{\prime}$ for all $u, v, \in[\lambda]^{<\aleph_{0}}$.

Fact 7.3 (Separation of variables theorem, special case, [20] Theorem 6.13). In the notation of [7.2, $\left\langle B_{u}: u \in[\kappa]^{<\aleph_{0}}\right\rangle$ has a multiplicative refinement in $\mathcal{D}$ [so $p$ is realized in $N]$ if and only if $\left\langle\mathbf{b}_{u}: u \in[\kappa]^{<\aleph_{0}}\right\rangle$ has a multiplicative refinement in $\mathcal{D}_{*}$.

Given $\mathfrak{B}$ and $\mathcal{D}$, it can be useful to remember an ultrapower it came from.

Notation 7.4. Given some $\mathcal{D}_{*}$ and $\mathfrak{B}$, and given a corresponding choice of $\mathcal{D}, \mathcal{D}_{0}$, and $M$ as in 7.2. we may call $M^{\lambda} / \mathcal{D}$ an "enveloping ultrapower" for $\mathcal{D}_{*}$ and $\mathfrak{B}$.

In light of 7.2 , 7.1 allows us to build regular ultrafilters in interesting ways by focusing on the construction of ultrafilters on quite general Boolean algebras: such a $\mathcal{D}_{0}$ can always be built when $\mathfrak{B}$ is complete, $|\mathfrak{B}| \leq 2^{\lambda}$ and has the $\lambda^{+}$-c.c. In earlier papers, $\mathfrak{B}$ was often just the completion of some free Boolean algebra. In this paper we take this quite a bit further, building the Boolean algebras and the ultrafilters on them together, by induction.

Possibility patterns. Definition 7.2 highlights sequences $\left\langle\mathbf{b}_{u}: u \in[\kappa]^{\left\langle\aleph_{0}\right.}\right\rangle$ of elements of $\mathfrak{B}^{+}$which come directly from patterns of types in some enveloping ultrapower. In [20] and subsequent papers, we use possibility pattern to mean any sequence $\overline{\mathbf{b}}$ that arises from some $\bar{B}$ in this way. There is a combinatorial definition which doesn't rely on specifying an enveloping ultrapower, see 20] Definition 6.1. In the present paper, we will only need to handle sequences $\overline{\mathbf{b}}$ which obviously come directly from types, so we won't need the full generality of that definition. So while we will often call such sequences "possibility patterns," the reader may substitute a phrase like "sequences $\overline{\mathbf{b}}$ which represent some $\varphi$-type $p$ in some enveloping ultrapower of the theory in question, in the sense of 7.2 ,"

Convention 7.5. When building Boolean algebras by induction, possibility patterns will arise as part of the data of problems, and we will often refer to multiplicative refinements we add for them as solutions.

In the proofs below, we use 7.1 to repeatedly calibrate the building of a Boolean algebra $\mathfrak{B}$ along with an ultrafilter $\mathcal{D}_{*}$ on $\mathfrak{B}$, as follows. Suppose we are building $\mathcal{D}_{*}$ and $\mathfrak{B}$ together by induction, and at each stage in the construction we have some Boolean algebra $\mathfrak{B}_{\alpha}$ and some ultrafilter $\mathcal{D}_{\alpha}$ on $\mathfrak{B}_{\alpha}$, and we want to ensure by the time we get to $\mathcal{D}_{*}, \mathfrak{B}$ that all relevant possibility patterns $\overline{\mathbf{b}}$, for a given countable theory $T$ or set $\mathcal{T}$ of countable theories, have multiplicative refinements. At each stage in the construction we'll be handling some such $\overline{\mathbf{b}}$, and at that point we may choose some enveloping ultrapower (for $\mathfrak{B}_{\alpha}, \mathcal{D}_{\alpha}$ ) and work there with a choice of corresponding $\bar{B}$, where the picture may be clearer. In this way we eventually construct $\mathfrak{B}$ and $\mathcal{D}_{*}$ to handle all relevant possibility patterns. At the end of the construction, one final use of 7.1 connects it to Keisler's order: any regular $\mathcal{D}$ built from this $\mathcal{D}_{*}$ will be a regular ultrafilter on $\lambda$ with the property that for any $T \in \mathcal{T}$ and any model $M \models T, M^{\lambda} / \mathcal{D}$ is $\lambda^{+}$-saturated.

Convention 7.6. For Boolean algebras, write that $\mathfrak{B}_{1} \lessdot \mathfrak{B}_{2}$ to mean that $\mathfrak{B}_{1}$ is a complete subalgebra of $\mathfrak{B}_{2}$.

Fact 7.7. If $\overline{\mathbf{b}}$ is a possibility pattern for $T$ in a complete Boolean algebra $\mathfrak{B}$, and $\mathfrak{B} \lessdot \mathfrak{B}^{\prime}$, then $\overline{\mathbf{b}}$ remains a possibility pattern for $T$ in $\mathfrak{B}^{\prime}$. 
Notation 7.8. In the context of separation of variables:

(a) Let $\left\langle B_{u}: u \in[\lambda]^{<\aleph_{0}}\right\rangle$ be from the Eoś map, as usual, recalling $[7.2$.

(b) For $\psi[\bar{a}]$ any formula, possibly with parameters, of $M^{I} / \mathcal{D}$, let $A[\psi[\bar{a}]]$ be defined as

$$
\{t \in I: M \models \psi[\bar{a}[t]]\} .
$$

(c) Given $B_{u}$ or $A[\psi[\bar{a}]]$ from (a) or $(b)$, let $\mathbf{b}_{u}$ or $\mathbf{a}[\psi[\bar{a}]]$ be their images under $\mathbf{j}$, respectively.

Together, we might say that the $\mathbf{b}_{u}$ 's and the $\mathbf{a}[\psi]$ 's transfer first-order information from the ultrapower to the index models (via Łos' theorem) and thence to the Boolean algebra (via $\mathbf{j}$ ). We conclude the section by restating 7.3 in this language:

Fact 7.9. Suppose $N=M^{\lambda} / \mathcal{D}$ is an "enveloping ultrapower" for $\mathcal{D}_{*}$ and $\mathfrak{B}$, $N \models T$, and $\overline{\mathbf{b}}=\left\langle\mathbf{b}_{u}: u \in[\kappa]^{<\aleph_{0}}\right\rangle$ is a possibility pattern for $T$ in $\mathfrak{B}$ which has no solution, i.e. no multiplicative refinement in $\mathcal{D}_{*}$. Then $N$ is not $\kappa^{+}$-saturated (specifically, it omits a type over a set of size $\leq \kappa$ corresponding naturally to $\overline{\mathbf{b}}$ ).

\section{The Chain CONDITION}

This section begins work on the ultrafilters. To motivate the construction, consider the limitations of the example of [28, the one previous example of incomparability in ZFC. In that paper, as usual, we had considered completions of free Boolean algebras of the form

$$
\mathfrak{B}=\mathfrak{B}_{2^{\lambda}, \mu, \theta}^{1}
$$

where $\theta$ was not necessarily countable [recall the notation means: $\mathfrak{B}$ is generated by $2^{\lambda}$ independent partitions each of size $\mu$, where the intersection of fewer than $\theta$ elements from different partitions is nonzero]. It was shown there that theories called $T_{f}$, distant precursors of our theories here, could be handled by some ultrafilter on $\mathfrak{B}$ when $\theta>\aleph_{0}$, and by no ultrafilter when $\theta=\aleph_{0}$. This suggests that if we want to handle theories of this general form while keeping $\theta=\aleph_{0}$, we should use Boolean algebras which are in a strong sense not free. In our present case, if we want to handle some $T_{\mathfrak{m}}$ while not handling another $T_{\mathfrak{n}}$, for $\mathfrak{m}, \mathfrak{n}$ orthogonal, perhaps we can start the induction with the completion of a free Boolean algebra and keep enough of the freeness for some failure of saturation for $T_{\mathfrak{n}}$ to persist even as we build the Boolean algebra to be gradually less free in a sense relevant to $T_{\mathfrak{m}}$ (adding formal solutions below). To successfully carry this out, the arbiter of the freeness we need will come in the form of a key chain condition $8.2{ }^{16}$

Specifically, in this section, we first define and explain the new chain condition in 8.2. Then, since in later sections we will build Boolean algebras by induction (as in 8.17), we will need some tools for ensuring the chain condition is preserved under such constructions. We define a so-called pattern transfer property in 8.12 using this property and 8.18, we prove Lemma 8.19, which verifies we may preserve our chain condition along certain increasing continuous sequences of Boolean algebras.

We continue notation from Section 96 . Recall:

\footnotetext{
${ }^{16}$ Although this is not needed for reading the proof, readers who are set theorists may recognize in the chain condition some methods intimately connected to finite support iteration (and may also conjecture that there may be potential for very interesting further interaction here).
} 
Definition 8.1. Let $\kappa$ be an uncountable regular cardinal. The Boolean algebra $\mathfrak{B}$ has the $\kappa$-c.c. when: given $\left\langle\mathbf{a}_{\alpha}: \alpha<\kappa\right\rangle$ a sequence of elements of $\mathfrak{B}^{+}$, we can find $\alpha \neq \beta<\kappa$ such that $\mathbf{a}_{\alpha} \cap \mathbf{a}_{\beta}>0$.

The following definition will be central for the next few sections.

Definition 8.2 (The $(\kappa, \mathcal{I}, \bar{m})$-c.c.). Let $\kappa$ be an uncountable regular cardinal. Let $\mathcal{I}$ be an ideal on $\omega$ extending $[\omega]^{<\aleph_{0}}$ and $\bar{m}$ a fast sequence. We say that the Boolean algebra $\mathfrak{B}$ has the $(\kappa, \mathcal{I}, \bar{m})$-c.c. when: given $\left\langle\mathbf{a}_{\alpha}: \alpha \in \mathcal{U}_{2}\right\rangle$ with $\mathcal{U}_{2} \in[\kappa]^{\kappa}$ a sequenc ${ }^{17}$ of elements of $\mathfrak{B}^{+}$, we can find $j<\omega, \mathcal{U}_{1} \in[\kappa]^{\kappa}$ and $A \in \mathcal{I}$ such that:

$\oplus$ for every $n \in \omega \backslash A$ and every finite $u \subseteq \mathcal{U}_{1}$ and every $i<n-j$,

if

$$
\frac{m_{n}}{\left(m_{n}^{\circ}\right)^{n^{i}}}<|u| \leq m_{n}
$$

then there is some $v \subseteq u$ such that

$$
|v| \geq \frac{|u|}{\left(m_{n}^{\circ}\right)^{n^{i+j}}} \text { and } \bigcap\left\{\mathbf{a}_{\alpha}: \alpha \in v\right\}>0_{\mathfrak{B}} .
$$

Observation 8.3. If $\mathfrak{B}$ has the $(\kappa, \mathcal{I}, \bar{m})$-c.c. for some $\mathcal{I}$ and $\bar{m}$, then $\mathfrak{B}$ has the $\kappa$-c.c. In particular, if $\kappa=\aleph_{1}$, then $\mathfrak{B}$ has the c.c.c.

Proof. Clearly, the condition in 8.2 implies that given any $\kappa$ nonzero elements of the Boolean algebra, at least two of them must have nonzero intersection.

Discussion 8.4. Informal discussion of the $(\kappa, \mathcal{I}, \bar{m})$-c.c. For any $n$, remember from 6.2 that any $u$ such that

$$
|u| \geq \frac{m_{n}}{\left(m_{n}^{\circ}\right)^{n^{n}}}
$$

is large, so if $|u| \geq m_{n} /\left(m_{n}^{\circ}\right)^{n^{i+j}}$ for some $i+j<n$, then $u$ is, by monotonicity, still large. In the statement of 8.2 we use $\mathcal{U}_{2}$ and $\mathcal{U}_{1}$ for easy quotation later on, but of course without loss of generality (after renumbering) $\mathcal{U}_{2}=\kappa$. In this notation, given any sequence of $\kappa$ nonzero elements, after possibly moving to a subsequence $\mathcal{U}$ of the same size, we can find a "shrinking factor" $j$ so that (after possibly excluding a small set of $n$ 's) whenever we have a really large $u$, i.e. $|u| \geq m_{n} /\left(m_{n}^{\circ}\right)^{n^{i}}$ for some $i$ such that $i+j$ is still $<n$, then we can shrink it a little bit (in the denominator the exponent $n^{i}$ becomes $n^{i+j}$ ) to find a subset $v$ which is still large, and all consistent.

The next claim shows that our upgraded c.c. is sometimes easy to satisfy. Recall from 1.7 that $\mathfrak{B}_{\alpha, \mu, \aleph_{0}}^{0}$ is the free Boolean algebra generated by $\alpha$ independent partitions each of size $\mu$, and $\mathfrak{B}_{\alpha, \mu, \aleph_{0}}^{1}$ is its completion.

Claim 8.5. Suppose $\mu$ is any infinite cardinal, $\kappa$ is regular and uncountable, $\alpha$ is an ordinal, and $\mu<\kappa \leq \alpha$. Then for any ideal $\mathcal{I}$ on $\omega$ extending $[\omega]<\aleph^{<}$, and any fast sequence $\bar{m}, \mathfrak{B}=\overline{\mathfrak{B}}_{\alpha, \mu, \aleph_{0}}^{1}$ has the $(\kappa, \mathcal{I}, \overline{\mathfrak{m}})$-c.c.

Proof. Recall from 1.10 above that the elements of the form $\mathbf{x}_{f}$ for $f \in \mathrm{FI}_{\aleph_{0}}(\alpha)$ are dense in $\mathfrak{B}$. Suppose we are given $\left\langle\mathbf{a}_{\alpha}: \alpha<\kappa\right\rangle$ a sequence of positive elements of $\mathfrak{B}$. First, for each $\alpha<\kappa$, we may choose $f_{\alpha} \in \mathrm{FI}_{\aleph_{0}}(\alpha)$ such that $\mathbf{x}_{f_{\alpha}} \leq \mathbf{a}_{\alpha}$. For each $\alpha<\kappa$, let $u_{\alpha}=\operatorname{dom}\left(f_{\alpha}\right)$, so $u_{\alpha}$ is finite. By the $\Delta$-system lemma 1.12, there is some $u_{*}$ and $\mathcal{V} \in[\kappa]^{\kappa}$ such that $u_{\alpha} \cap u_{\beta}=u_{*}$ for $\alpha, \beta \in \mathcal{V}$. Since the range of

\footnotetext{
${ }^{17}$ or renaming, without loss of generality, $\mathcal{U}_{2}=\kappa$.
} 
each $f_{\alpha}$ is a subset of $\mu<\kappa$, there is $\mathcal{U} \in[\mathcal{V}]^{\kappa}$ such that $f_{\alpha}\left\lceil u_{*}=f_{\beta}\left\lceil u_{*}\right.\right.$ for $\alpha, \beta \in \mathcal{U}$. Notice this tells us for any finitely many $\alpha_{0}, \ldots, \alpha_{n-1}$ from $\mathcal{U}, f=\cup_{i<n} f_{i}$ is a function, thus $\mathbf{x}_{f}>0$. In other words, for any finite $v \subseteq \mathcal{U}$,

$$
\bigcap\left\{\mathbf{a}_{\alpha}: \alpha \in v\right\}>0_{\mathfrak{B}}
$$

so condition is trivially satisfied [indeed, taking $A=\emptyset$ and $j=0$ ] for any $n$ and $u$, simply by using $v=u$.

Remark 8.6. We have stated 8.5 to be compliant with Definition 8.2. but notice the proof would go through for any ideal $\mathcal{I}$ including the trivial ideal $\emptyset$.

Starting with the next Discussion, we add asterisks to a few statements and definitions to indicate that they are useful variations.

${ }^{\star}$ Discussion 8.7. In the next Definition 8.8 note we replace $\mu$ in the subscript by " $<\kappa$ " (it will be explained what this means). This includes the natural case where $\kappa$ is a regular limit cardinal, thus weakly inaccessible. This gives a natural alternate candidate for $\mathfrak{B}_{*}$ in $9.1(2)$, so we include it, but again, it is not our main case. For our main theorems, the case $\kappa=\mu^{+}$, and thus using as a base for our induction Boolean algebras of the form $\mathfrak{B}_{\alpha, \mu, \aleph_{0}}^{1}$, or even $\mathfrak{B}_{\kappa, \aleph_{0}, \aleph_{0}}^{1}$, will suffice.

${ }^{\star}$ Definition 8.8. Let $\theta=\operatorname{cof}(\theta) \leq \kappa=\operatorname{cof}(\kappa)$ and let $\alpha$ be an ordinal.

(1) $\mathcal{F}_{\alpha,<\kappa, \aleph_{0}}=\left\{f: \operatorname{dom}(f) \in[\alpha]^{<\theta}\right.$, and if $\beta \in \operatorname{dom}(f)$ then $\left.f(\beta)<\operatorname{rm}_{\kappa}(\beta)\right\}$ where $\operatorname{rm}_{\kappa}(\beta)=\min \left\{i: \bigvee_{\gamma}(\beta=\kappa \cdot \gamma+i)\right\}$ is the remainder of $\beta \bmod \kappa$.

(2) $\mathfrak{B}_{\alpha,<\kappa, \theta}^{0}$ is the Boolean algebra generated by $\left\{\mathbf{x}_{f}: f \in \mathcal{F}_{\alpha,<\kappa, \theta}\right\}$ freely except:

(a) $\mathbf{x}_{f} \leq \mathbf{x}_{g}$ when $g \subseteq f \in \mathcal{F}_{\alpha,<\kappa, \theta}$

(b) $\mathbf{x}_{f} \cap \mathbf{x}_{g}=0$ when $(\exists \beta \in \operatorname{dom}(f) \cap \operatorname{dom}(g))[f(\beta) \neq g(\beta)]$.

(3) Let $\mathfrak{B}_{\alpha,<\kappa, \theta}=\mathfrak{B}_{\alpha,<\kappa, \theta}^{1}$ be the completion of $\mathfrak{B}_{\alpha,<\kappa, \theta}^{0}$.

We verify that Claim 8.5 likewise holds for $\mathfrak{B}_{\alpha,<\kappa, \theta}$.

${ }^{\star}$ Claim 8.9. Suppose $\kappa$ is regular and uncountable and $\alpha \in$ Ord. Suppose that in addition $(\forall \alpha<\kappa)\left(\alpha^{<\theta}<\kappa\right)$. Then $\mathfrak{B}_{\alpha,<\kappa, \aleph_{0}}^{0}$ and $\mathfrak{B}=\mathfrak{B}_{\alpha,<\kappa, \theta}^{1}$ satisfy the $\kappa$-c.c., and indeed the $(\kappa, \mathcal{I}, \bar{m})$-c.c. for any $\bar{m}$ and $\mathcal{I}$.

Proof. Similarly to 8.5. suppose we are given $\left\langle\mathbf{a}_{\alpha}: \alpha<\kappa\right\rangle$ a sequence of positive elements of $\mathfrak{B}$. For each $\alpha<\kappa$, we may choose $f_{\alpha} \in \mathcal{F}_{\alpha,<\kappa, \aleph_{0}}$, so $u_{\alpha}=\operatorname{dom}\left(f_{\alpha}\right) \in$ $[\alpha]^{<\theta}$, such that $\mathbf{x}_{f_{\alpha}} \leq \mathbf{a}_{\alpha}$. Assuming that $(\forall \alpha<\kappa)\left(\alpha^{<\nu}<\kappa\right)$, the hypotheses of the $\Delta$-system lemma 1.11 apply, and there is some $u_{*}$ and $\mathcal{V} \in[\kappa]^{\kappa}$ such that $u_{\alpha} \cap u_{\beta}=u_{*}$ for $\alpha, \beta \in \mathcal{V}$. Let $\gamma=\sup \left\{\operatorname{rm}_{\kappa}(\beta): \beta \in u_{*}\right\}$, so $\gamma<\kappa$ as $\left|u_{*}\right|<\aleph_{0}$. By Definition $8.8(1)$, each $f_{\alpha}\left\lceil u_{*}\right.$ has range $\subseteq \gamma+1<\kappa$, so there is $\mathcal{U} \in[\mathcal{V}]^{\kappa}$ such that $f_{\alpha}\left\lceil u_{*}=f_{\beta}\left\lceil u_{*}\right.\right.$ for $\alpha, \beta \in \mathcal{U}$. So just as before, for any finite $v \subseteq \mathcal{U}$,

$$
\bigcap\left\{\mathbf{a}_{\alpha}: \alpha \in v\right\}>0_{\mathfrak{B}}
$$

so condition for the $(\kappa, \mathcal{I}, \bar{m})$-c.c. is trivially satisfied for any $n$ and $u$, simply by using $v=u$.

Recall from 7.6 that $\mathfrak{B}_{1} \lessdot \mathfrak{B}_{2}$ means $\mathfrak{B}_{1}$ is a complete subalgebra of $\mathfrak{B}_{2}$.

In our inductive construction, the so-called "pattern transfer property" (Definition 8.12 below) will play a key role in ensuring that the $(\kappa, \mathcal{I}, \bar{m})$-c.c. is preserved at limit stages. In some sense, it shows a close connection between a smaller and larger Boolean algebra which is enough to allow the $(\kappa, \mathcal{I}, \bar{m})$-c.c. to transfer from the smaller to the larger one. We first need a definition. 
Definition 8.10. Let $\mathfrak{B}_{1} \lessdot \mathfrak{B}_{2}$ and let $\mathbf{b} \in \mathfrak{B}_{2}^{+}$. We say that $\mathbf{a} \in \mathfrak{B}_{1}^{+}$is below the projection of $\mathbf{b}$, in symbols

$$
\mathbf{a} \leq_{\operatorname{proj}\left(\mathfrak{B}_{2}, \mathfrak{B}_{1}\right)} \mathbf{b}
$$

when for every $\mathbf{c} \in \mathfrak{B}_{1}^{+}$such that $\mathfrak{B}_{1} \models \mathbf{c} \leq \mathbf{a}$, we have that $\mathfrak{B}_{2} \models \mathbf{c} \cap \mathbf{b}>0$. When $\mathfrak{B}_{2}, \mathfrak{B}_{1}$ are clear from context, we may omit them from the subscript.

Observation 8.11. If $\mathfrak{B}_{1} \lessdot \mathfrak{B}_{2} \lessdot \mathfrak{B}_{3}, \mathbf{a} \in \mathfrak{B}_{1}^{+}, \mathbf{b} \in \mathfrak{B}_{2}^{+}, \mathbf{c} \in \mathfrak{B}_{3}^{+}$,

$$
\left(\mathbf{a} \leq_{\operatorname{proj}\left(\mathfrak{B}_{2}, \mathfrak{B}_{1}\right)} \mathbf{b} \leq_{\operatorname{proj}\left(\mathfrak{B}_{3}, \mathfrak{B}_{2}\right)} \mathbf{c}\right) \Longrightarrow\left(\mathbf{a} \leq_{\operatorname{proj}\left(\mathfrak{B}_{3}, \mathfrak{B}_{1}\right)} \mathbf{c}\right) .
$$

Proof. Given $\mathbf{c}_{1} \in \mathfrak{B}_{1}^{+}$such that $\mathfrak{B}_{1} \models \mathbf{c}_{1} \leq \mathbf{a}$, the first inequality ensures that $\mathfrak{B}_{2} \models \mathbf{c}_{1} \cap \mathbf{b}>0$. Let $\mathbf{c}_{2}:=\mathbf{c}_{1} \cap \mathbf{b} \in \mathfrak{B}_{2}^{+}$, so $\mathfrak{B}_{2} \models \mathbf{c}_{2} \leq \mathbf{b}$, and the second inequality ensures that $\mathfrak{B}_{3} \models \mathbf{c}_{2} \cap \mathbf{c}>0$, thus (since $0<\mathbf{c}_{2} \leq \mathbf{c}_{1}$ ) $\mathfrak{B}_{3} \models \mathbf{c}_{1} \cap \mathbf{c}>0$.

Definition 8.12 (Pattern transfer property). Let $\kappa$ be an uncountable cardinal, $\mathcal{I}$ an ideal on $\omega$ extending $[\omega]<\aleph_{0}$, and $\bar{m}$ a fast sequence. The pair $\left(\mathfrak{B}_{1}, \mathfrak{B}_{2}\right)$ has the $(\kappa, \mathcal{I}, \bar{m})$-pattern transfer property when: $(\mathbf{1}) \mathfrak{B}_{1}$ and $\mathfrak{B}_{2}$ are both complete Boolean algebras, (2) $\mathfrak{B}_{1}$ satisfies the $\kappa$-c.c 18 , $(\mathbf{3}) \mathfrak{B}_{1} \lessdot \mathfrak{B}_{2}$, and $(\mathbf{4})$ whenever $\mathcal{U}_{2} \in[\kappa]^{\kappa}$ and $\overline{\mathbf{b}}=\left\langle\mathbf{b}_{\alpha}: \alpha \in \mathcal{U}_{2}\right\rangle$ is a sequence of elements of $\mathfrak{B}_{2}^{+}$, we can find a quadruple $\left(j, \mathcal{U}_{1}, A, \overline{\mathbf{a}}\right)$ such that:

(a) $j<\omega$

(b) $\mathcal{U}_{1} \in\left[\mathcal{U}_{2}\right]^{\kappa}$

(c) $A \in \mathcal{I}$

(d) $\overline{\mathbf{a}}=\left\langle\mathbf{a}_{\alpha}: \alpha \in \mathcal{U}_{1}\right\rangle$ is a sequence of distinct elements of $\mathfrak{B}_{1}^{+}$

(e) $\alpha \in \mathcal{U}_{1}$ implies $\mathbf{a}_{\alpha} \leq_{\text {proj }} \mathbf{b}_{\alpha}$

(f) (i) implies (ii) where:

(i) we are given $n \in \omega \backslash A, i+j<n, u \subseteq \mathcal{U}_{1}$, and $\mathbf{a}_{*} \in \mathfrak{B}_{1}^{+}$such that $m_{n} /\left(m_{n}^{\circ}\right)^{n^{i}}<|u|<m_{n}$ and

$$
\mathfrak{B}_{1} \models \mathbf{a}_{*} \leq \bigcap_{\alpha \in u} \mathbf{a}_{\alpha}
$$

(ii) there is $v$ such that $v \subseteq u$ and $|v| \geq|u| /\left(m_{n}^{\circ}\right)^{n^{i+j}}$ and

$$
\mathfrak{B}_{2} \models \bigcap_{\alpha \in v} \mathbf{b}_{\alpha} \cap \mathbf{a}_{*}>0 .
$$

Remark 8.13. One reason $8.12(4)(f)$ is not trivial is that $\mathbf{a}_{\alpha}^{1} \leq_{\text {proj }} \mathbf{a}_{\alpha}^{2}$ does not imply $\mathbf{a}_{\alpha}^{1} \leq \mathbf{a}_{\alpha}^{2}$.

Observation $\mathbf{8 . 1 4}$ (The pattern transfer property is transitive). Suppose

$$
\mathfrak{B}_{1} \lessdot \mathfrak{B}_{2} \lessdot \mathfrak{B}_{3}
$$

are complete Boolean algebras and the pairs $\left(\mathfrak{B}_{1}, \mathfrak{B}_{2}\right)$ and $\left(\mathfrak{B}_{2}, \mathfrak{B}_{3}\right)$ have the $(\kappa, \mathcal{I}, \bar{m})$ pattern transfer property. Then so does $\left(\mathfrak{B}_{1}, \mathfrak{B}_{3}\right)$.

Proof. We start with $\mathcal{U}_{3} \in[\kappa]^{\kappa}$ and $\overline{\mathbf{c}}=\left\langle\mathbf{c}_{\alpha}: \alpha \in \mathcal{U}_{3}\right\rangle$ in $\mathfrak{B}_{3}^{+}$. Applying the pattern transfer property for $\left(\mathfrak{B}_{2}, \mathfrak{B}_{3}\right)$ to $\overline{\mathbf{c}}$, we find $j_{2}, \mathcal{U}_{2}, A_{2}$, and a sequence $\overline{\mathbf{b}}=\left\langle\mathbf{b}_{\alpha}: \alpha \in \mathcal{U}_{2}\right\rangle$ of elements of $\mathfrak{B}_{2}^{+}$. Next, applying the pattern transfer property

\footnotetext{
${ }^{18}$ In this definition, we don't ask that $\mathfrak{B}_{1}$ have the $(\kappa, \mathcal{I}, \bar{m})$-c.c., only the $\kappa$-c.c., though in every application in the paper, $\mathfrak{B}_{1}$ will have the $(\kappa, \mathcal{I}, \bar{m})$-c.c.
} 
for $\left(\mathfrak{B}_{1}, \mathfrak{B}_{2}\right)$ to $\overline{\mathbf{b}}$ [which, note, is indexed by $\mathcal{U}_{2}$, so we will get $\mathcal{U}_{1} \subseteq \mathcal{U}_{2}$ ] we find $j_{1}, \mathcal{U}_{1}, A_{1}$, and a sequence $\left\langle\mathbf{a}_{\alpha}: \alpha \in \mathcal{U}_{1}\right\rangle$ of elements of $\mathfrak{B}_{1}^{+}$. In short,

$$
\begin{array}{r}
\left\langle\mathbf{c}_{\alpha}: \alpha \in \mathcal{U}_{3}\right\rangle \text { from } \mathfrak{B}_{3}^{+} \\
\downarrow \text { via }\left(j_{2}, \mathcal{U}_{2}, A_{2}\right) \\
\left\langle\mathbf{b}_{\alpha}: \alpha \in \mathcal{U}_{2}\right\rangle \text { from } \mathfrak{B}_{2}^{+} \\
\quad \downarrow \text { via }\left(j_{1}, \mathcal{U}_{1}, A_{1}\right) \\
\left\langle\mathbf{a}_{\alpha}: \alpha \in \mathcal{U}_{1}\right\rangle \text { from } \mathfrak{B}_{1}^{+} .
\end{array}
$$

Let $j=j_{1}+j_{2}+1$ and $A=A_{1} \cup A_{2} \cup\{0,1\}$, and we shall verify that

$$
\begin{aligned}
\left\langle\mathbf{c}_{\alpha}: \alpha \in \mathcal{U}_{3}\right\rangle \text { from } \mathfrak{B}_{3}^{+} & \\
& \downarrow \text { via }\left(j, \mathcal{U}_{1}, A\right) \\
\left\langle\mathbf{a}_{\alpha}:\right. & \left.\alpha \in \mathcal{U}_{1}\right\rangle \text { from } \mathfrak{B}_{1}^{+}
\end{aligned}
$$

The point to check is $8.12(4)$. Conditions (a)-(d) are clear, condition (e) is by 8.11, so it remains to verify (f)(i) $\rightarrow$ (ii). Suppose we are given $n, i, u, \mathbf{a}_{*}$ such that $n \in \omega \backslash A, i+j<n, u \subseteq \mathcal{U}_{1}, \mathbf{a}_{*} \in \mathfrak{B}_{1}^{+}, m_{n} /\left(m_{n}^{\circ}\right)^{n^{i}}<|u|<m_{n}$, and $\mathfrak{B}_{1} \models \mathbf{a}_{*} \leq \bigcap_{\alpha \in u} \mathbf{a}_{\alpha}$. Since $i+j_{1}<i+j<n$, we may apply the pattern transfer from $\mathfrak{B}_{1}$ to $\mathfrak{B}_{2}$ to this data to find $v^{\prime} \subseteq u$ such that $\left|v^{\prime}\right| \geq|u| /\left(m_{n}^{\circ}\right)^{n^{i+j_{1}}}$ and $\mathfrak{B}_{2} \models \bigcap_{\alpha \in v^{\prime}} \mathbf{b}_{\alpha} \cap \mathbf{a}_{*}>0$. Choose $a_{* *}$ to be any element of $\mathfrak{B}_{2}^{+}$below $\bigcap_{\alpha \in v^{\prime}} \mathbf{b}_{\alpha} \cap \mathbf{a}_{*}$. Note that $\left|v^{\prime}\right| \geq|u| /\left(m_{n}^{\circ}\right)^{n^{i+j_{2}}}$ and $|u|>m_{n} /\left(m_{n}^{\circ}\right)^{n^{i}}$ together imply

$$
\left|v^{\prime}\right|>\frac{m_{n}}{\left(m_{n}^{\circ}\right)^{n^{i}}\left(m_{n}^{\circ}\right)^{n^{i+j_{2}}}} \geq \frac{m_{n}}{\left(m_{n}^{\circ}\right)^{n^{i+j_{1}+1}}} .
$$

So we may apply the pattern transfer from $\mathfrak{B}_{2}$ to $\mathfrak{B}_{3}$ using the same $n$ and using $i+j_{1}+1, v^{\prime}$, and $\mathbf{a}_{* *}$ in place of $i, u, \mathbf{a}_{*}$ respectively in (f)(i). [Note that we have $\left(i+j_{1}+1\right)+j_{2} \leq i+j<n$ by our choice of $j$.] We obtain from (f)(ii) a subset $v \subseteq v^{\prime}$ [thus, $\subseteq u$ ] with $|v| \geq\left|v^{\prime}\right| /\left(m_{n}^{\circ}\right)^{n^{\left(i+j_{1}+1\right)+j_{2}}}$ such that $\mathfrak{B}_{3} \models \bigcap_{\alpha \in v} \mathbf{c}_{\alpha} \cap \mathbf{a}_{* *}>0$, and since $\mathbf{a}_{* *} \leq \mathbf{a}_{*}, \mathfrak{B}_{3} \models \bigcap_{\alpha \in v} \mathbf{c}_{\alpha} \cap \mathbf{a}_{*}>0$. This shows that for $\overline{\mathbf{c}}$ the quadruple $\left(j=j_{1}+j_{2}+1, \mathcal{U}, A, \overline{\mathbf{a}}\right)$ works, which completes the verification.

Claim 8.15 (The pattern transfer property indeed transfers our chain condition). Suppose $\mathfrak{B}_{1} \lessdot \mathfrak{B}_{2}, \mathfrak{B}_{1}$ has the $(\kappa, \mathcal{I}, \bar{m})$-c.c., and $\left(\mathfrak{B}_{1}, \mathfrak{B}_{2}\right)$ has the $(\kappa, \mathcal{I}, \bar{m})$-pattern transfer property. Then $\mathfrak{B}_{2}$ has the $(\kappa, \mathcal{I}, \bar{m})$-c.c.

Proof. Suppose we are given $\left\langle\mathbf{a}_{\alpha}^{2}: \alpha \in \mathcal{U}_{2}\right\rangle, \mathcal{U}_{2} \in[\kappa]^{\kappa}$ in $\mathfrak{B}_{2}$. Applying the pattern transfer property, we find $j_{1}, \mathcal{U}_{1}, A_{1}$, and $\left\langle\mathbf{a}_{\alpha}^{1}: \alpha \in \mathcal{U}_{1}\right\rangle$ in $\mathfrak{B}_{1}^{+}$satisfying 8.12 . Next, working in $\mathfrak{B}_{1}$, we apply the $(\kappa, \mathcal{I}, \bar{m})$-c.c. to the sequence $\left\langle\mathbf{a}_{\alpha}^{1}: \alpha<\kappa\right\rangle$ and find $j_{0}, \mathcal{U}_{0}, A_{0}$ satisfying 8.2 . Now let us check that $\mathfrak{B}_{2}$ satisfies the $(\kappa, \mathcal{I}, \bar{m})$-c.c. using $\mathcal{U}=\mathcal{U}_{0} \subseteq \mathcal{U}_{1} \subseteq \mathcal{U}_{2}, A=A_{1} \cup A_{0}$, and $j=j_{1}+j_{0}+2$. Suppose we are given $n \in \omega \backslash A$ and a finite $u \subseteq \mathcal{U}$. We know that if $i+j<n$ and

$$
\frac{m_{n}}{\left(m_{n}^{\circ}\right)^{n^{i}}}<|u| \leq m_{n}
$$

there is some $v^{\prime} \subseteq u$ such that in $\mathfrak{B}_{1}$,

$$
\left|v^{\prime}\right| \geq \frac{|u|}{\left(m_{n}^{\circ}\right)^{n^{i+j_{1}}}} \geq \frac{m_{n}}{\left(m_{n}^{\circ}\right)^{n^{i+j_{1}+1}}} \text { and } \mathfrak{B}_{1} \models \bigcap\left\{\mathbf{a}_{\alpha}^{1}: \alpha \in v^{\prime}\right\}>0 .
$$


Now 8.12 (f)(i) holds, using $v^{\prime}, i+j_{1}+1$ here for $u, i$ there. (Note: the identity of $\mathbf{a}_{*}$ in that equation is not important here; we'll use it later when we deal with omitting types.) So 8.12 (f)(ii) tells us there is $v \subseteq v^{\prime}$ such that $|v| \geq\left|v^{\prime}\right| /\left(m_{n}^{\circ}\right)^{n^{i+j_{1}+j_{0}+1}}$ and $\mathfrak{B}_{2} \models \bigcap_{\alpha \in v} \mathbf{a}_{\alpha}^{2}>0$. Checking the size,

$$
|v| \geq \frac{\left|v^{\prime}\right|}{\left(m_{n}^{\circ}\right)^{n^{i+j_{1}+j_{0}+1}}} \geq \frac{|u|}{\left(m_{n}^{\circ}\right)^{n^{i+j_{1}}}\left(m_{n}^{\circ}\right)^{n^{i+j_{1}+j_{0}+1}}} \geq \frac{|u|}{\left(m_{n}^{\circ}\right)^{n^{i+j_{1}+j_{0}+2}}} .
$$

This completes the verification.

We recall the following fact which we will upgrade to the case of our chain condition in 8.18

Fact 8.16 (Jech, [8] Corollary 16.10). Let $\mathfrak{B}_{0} \subseteq \mathfrak{B}_{1} \subseteq \cdots \subseteq \mathfrak{B}_{\beta} \subseteq \cdots(\beta<\alpha)$ be a sequence of complete Boolean algebras such that for all $\beta<\gamma, \mathfrak{B}_{\beta} \lessdot \mathfrak{B}_{\gamma}$, and for each limit ordinal $\gamma, \bigcup_{\beta<\gamma} \mathfrak{B}_{\beta}$ is dense in $\mathfrak{B}_{\gamma}$. If every $\mathfrak{B}_{\beta}$ satisfies the $\kappa$-c.c. then $\bigcup_{\beta<\alpha} \mathfrak{B}_{\beta}$ satisfies the $\kappa$-c.c.

Definition 8.17. We say that $\overline{\mathfrak{B}}=\left\langle\mathfrak{B}_{\gamma}: \gamma \leq \delta\right\rangle$ is a $\lessdot$-increasing continuous sequence of complete Boolean algebras when:

(1) for $\gamma<\delta, \mathfrak{B}_{\gamma} \lessdot \mathfrak{B}_{\gamma+1}$;

(2) at limit stages $\overline{\mathfrak{B}}$ is continuous, meaning that if $\gamma<\delta$ is a limit ordinal then $\bigcup\left\{\mathfrak{B}_{\gamma}: \gamma<\delta\right\}$ is a dense subset of $\mathfrak{B}_{\delta}$. (Requiring that the union is $\lessdot \mathfrak{B}_{\delta}$ is ok also.)

Our next claim 8.18 will carry the induction for Lemma 8.19

Claim 8.18. Suppose that $\overline{\mathfrak{B}}=\left\langle\mathfrak{B}_{\gamma}: \gamma \leq \delta\right\rangle$ is a of complete Boolean algebras, where:

(1) $\mathfrak{B}_{\gamma}$ satisfies the $(\kappa, \mathcal{I}, \bar{m})$-c.c. for every $\gamma<\delta$.

(2) if $\gamma<\delta$ then the pair $\left(\mathfrak{B}_{\gamma}, \mathfrak{B}_{\gamma+1}\right)$ satisfies the $(\kappa, \mathcal{I}, \bar{m})$-pattern transfer property.

(3) if $\gamma<\beta<\delta$ then the pair $\left(\mathfrak{B}_{\gamma}, \mathfrak{B}_{\beta}\right)$ satisfies the $(\kappa, \mathcal{I}, \bar{m})$-pattern transfer property.

Then $\mathfrak{B}_{\delta}$ satisfies the $(\kappa, \mathcal{I}, \bar{m})$-c.c., and moreover, if $\gamma<\delta$ then the pair $\left(\mathfrak{B}_{\gamma}, \mathfrak{B}_{\delta}\right)$ satisfies the $(\kappa, \mathcal{I}, \bar{m})$-pattern transfer property.

Proof. The case to consider is $\delta$ a nonzero limit. Fix $\gamma_{*}<\delta$, and we will show that $\left(\mathfrak{B}_{\gamma_{*}}, \mathfrak{B}_{\delta}\right)$ has the $(\kappa, \mathcal{I}, \bar{m})$-transfer property. We may then conclude by 8.15 that $\mathfrak{B}_{\delta}$ has the $(\kappa, \mathcal{I}, \bar{m})$-c.c.

Suppose we are given $\left\langle\mathbf{a}_{\alpha}^{\delta}: \alpha<\kappa\right\rangle$ a sequence of elements of $\mathfrak{B}_{\delta}^{+}$. Without loss of generality (by the definition of continuous) each $\mathbf{a}_{\alpha}^{\delta}$ is an element of $\bigcup_{\gamma<\delta} \mathfrak{B}_{\gamma}$ (i.e., each element is already a member of some $\mathfrak{B}_{\gamma}$ ).

Recall $\kappa$ is regular. If $\operatorname{cof}(\delta) \neq \kappa$, there is $\gamma_{* *}<\delta$ such that $\mathcal{U}=\left\{\alpha<\kappa: \mathbf{a}_{\alpha}^{\delta} \in\right.$ $\left.\mathfrak{B}_{\gamma_{* *}}\right\}$ has size $\kappa$. Since $\mathfrak{B}_{\gamma_{* *}}$ has the $(\kappa, \mathcal{I}, \bar{m})$-c.c. by hypothesis, $\mathfrak{B}_{\delta}$ will inherit it from the restriction to $\mathcal{U}$. Since without loss of generality $\gamma_{* *}>\gamma_{*}$, and $\left(\mathfrak{B}_{\gamma_{*}}, \mathfrak{B}_{\gamma_{* *}}\right)$ has the $(\kappa, \mathcal{I}, \bar{m})$-pattern transfer property by hypothesis, we can similarly see that $\left(\mathfrak{B}_{\gamma_{*}}, \mathfrak{B}_{\delta}\right)$ has the $(\kappa, \mathcal{I}, \bar{m})$-pattern transfer property too (by restricting to $\mathcal{U}$ ).

Suppose then that $\operatorname{cof}(\delta)=\kappa$. In this case fix a strictly increasing continuous sequence $\left\langle i_{\alpha}: \alpha<\kappa\right\rangle$ of ordinals $<\delta$ but above $\gamma_{*}$, with limit $\delta$. First we choose a sequence of elements $\left\langle\mathbf{b}_{\alpha}: \alpha<\kappa\right\rangle$, as follows. There is $\zeta(\alpha) \in(\alpha, \kappa)$ such that

$$
\mathbf{a}_{\alpha}^{\delta} \in \mathfrak{B}_{i_{\zeta(\alpha)}} .
$$


As $\alpha<\zeta(\alpha)$, by our assumption $\mathfrak{B}_{i_{\alpha}} \lessdot \mathfrak{B}_{i_{\zeta(\alpha)}}$. As $\mathbf{a}_{\alpha}^{\delta} \in \mathfrak{B}_{i_{\zeta(\alpha)}}$, by definition of $\lessdot$, we may find a $\mathbf{b}_{\alpha} \in \mathfrak{B}_{i_{\alpha}}^{+}$such that

$$
\mathbf{b}_{\alpha} \leq_{\operatorname{proj}\left(\mathfrak{B}_{i \zeta(\alpha)}, \mathfrak{B}_{i_{\alpha}}\right)} \mathbf{a}_{\alpha}^{\delta} .
$$

Let $\left\langle\mathbf{b}_{\alpha}: \alpha<\kappa\right\rangle$ be the sequence of nonzero elements defined in this way.

Next we choose a sequence of elements $\left\langle\mathbf{c}_{\epsilon}: \epsilon<\kappa\right.$ is a limit $\rangle$, as follows. For every limit ordinal $\epsilon<\kappa$, recall that $\bigcup_{\alpha<\epsilon} \mathfrak{B}_{i_{\alpha}}$ is dense in $\mathfrak{B}_{i_{\epsilon}}$ by definition of continuous. Recall that $\mathbf{b}_{\epsilon} \in \mathfrak{B}_{i_{\epsilon}}$ by construction (b). So for every limit ordinal $\epsilon<\kappa$, there is $\mathbf{c}_{\epsilon}$ such that $\mathbf{c}_{\epsilon} \in \bigcup_{\alpha<\epsilon} \mathfrak{B}_{i_{\alpha}}, \mathfrak{B}_{i_{\epsilon}} \models 0<\mathbf{c}_{\epsilon}<\mathbf{b}_{\epsilon}$, and (hence) $\mathbf{c}_{\epsilon} \in \mathfrak{B}_{i_{\rho(\epsilon)}^{+}}$for some $\rho(\epsilon)<\epsilon$. Now the function $\epsilon \mapsto \rho(\epsilon)$ is defined and regressive on the limit ordinals $<\kappa$, so by Fodor's lemma there is $\rho_{*}<\kappa$ such that $\mathcal{U}_{0}=$ $\left\{\epsilon<\kappa: \epsilon\right.$ is a limit $>\rho_{*}$, and $\left.\rho(\epsilon)<\rho_{*}\right\}$ is a stationary subset of $\kappa$, recalling that $\kappa=\operatorname{cof}(\kappa)>\aleph_{0}$ by 8.2 . Recalling $\zeta$ from (国), let $E$ be a club of $\kappa$ such that $\epsilon<\beta \in E$ implies $\zeta(\epsilon)<\beta$. Then

$$
\mathcal{U}=E \cap \mathcal{U}_{0}
$$

is a stationary subset of $\kappa$, hence of size $\kappa$. [For orientation: for $\epsilon \in E$, the $\mathbf{c}_{\epsilon}$ 's all belong to $\mathfrak{B}_{i_{\rho_{*}}}$, while $\mathbf{b}_{\epsilon} \in \mathfrak{B}_{i_{\epsilon}}, \mathbf{a}_{\epsilon}^{\delta} \in \mathfrak{B}_{i_{\zeta(\epsilon)}}$, and recall $\rho_{*}<\epsilon<\zeta(\epsilon)<\kappa$, while $i_{\rho_{*}}<i_{\epsilon}<i_{\zeta(\epsilon)}<i_{\kappa}=\delta$. Moreover, if $\gamma<\epsilon$ are from $\mathcal{U}$, then $\mathfrak{B}_{i_{\zeta(\epsilon)}}$ already contains $\mathbf{b}_{\gamma}$ and $\mathbf{a}_{\gamma}^{\delta}$.]

We will now show the following relationship between $\left\langle\mathbf{c}_{\epsilon}: \epsilon \in \mathcal{U}\right\rangle$ and $\left\langle\mathbf{a}_{\epsilon}^{\delta}: \epsilon \in \mathcal{U}\right\rangle$ : for any finite $u \subseteq \mathcal{U}$,

$$
\mathfrak{B}_{i_{* *}} \models \bigcap_{\alpha \in u} \mathbf{c}_{\alpha}>0 \Longrightarrow \mathfrak{B}_{\delta} \models \bigcap_{\alpha \in u} \mathbf{a}_{\alpha}^{\delta}>0 .
$$

Let $n=|u|$, and let $\epsilon(0)<\cdots<\epsilon(n-1)$ list $u$. By induction on $m \leq n$, let us show that

$$
(\star)_{m} \text { if } \mathfrak{B}_{i_{\rho_{*}}} \models \bigcap_{\ell<m} \mathbf{c}_{\epsilon(\ell)} \geq \mathbf{d}>0\left[\text { so } \mathbf{d} \in \mathfrak{B}_{i_{\rho_{*}}}\right] \text { then } \mathfrak{B}_{\delta} \models \bigcap_{\ell<m} \mathbf{a}_{\epsilon(\ell)}^{\delta} \cap \mathbf{d}>0 .
$$

Clearly for $m=n$ this will imply equation (d). Note that for $m=0$, the intersections $\bigcap_{\ell<0} \mathbf{c}_{\epsilon(\ell)}$ and $\bigcap_{\ell<0} \mathbf{a}_{\epsilon(\ell)}^{\delta}$ are $1_{\mathfrak{B}_{\rho_{*}}}$ and $1_{\mathfrak{B}_{\delta}}$ respectively. For $m=1$, we are assuming $\mathfrak{B}_{i_{\rho_{*}}} \models 0<\mathbf{d} \leq \mathbf{c}_{\epsilon(0)}$ thus $\mathfrak{B}_{i_{\epsilon(0)}} \models 0<\mathbf{d} \leq \mathbf{c}_{\epsilon(0)} \leq \mathbf{b}_{\epsilon(0)}$, so by equation (b), $\mathfrak{B}_{i_{\zeta(\epsilon(0))}} \models 0<\mathbf{d} \cap \mathbf{a}_{\epsilon(0)}^{\delta}$, and thus $\mathfrak{B}_{\delta} \models 0<\mathbf{d} \cap \mathbf{a}_{\epsilon(0)}^{\delta}$, proving $(\star)_{1}$. For the inductive step, assume $m<n$ and that $(\star)_{m}$ holds and we shall prove $(\star)_{m+1}$.

For $(\star)_{m+1}$, we are assuming $\mathfrak{B}_{i_{\rho_{*}}} \models \bigcap_{\ell \leq m} \mathbf{c}_{\epsilon(\ell)} \geq \mathbf{d}>0$. By inductive hypothesis, $\mathfrak{B}_{\delta} \models \mathbf{d}^{\prime}=\bigcap_{\ell<m} \mathbf{a}_{\epsilon(\ell)}^{\delta} \cap \mathbf{d}>0$. Now $\left\{\mathbf{a}_{\epsilon(\ell)}^{\delta}: \ell<m\right\} \cup\{\mathbf{d}\} \subseteq \bigcup\left\{\mathfrak{B}_{i_{\zeta(\epsilon(\ell))}}: \ell<\right.$ $m\} \cup \mathfrak{B}_{i_{\rho_{*}}} \subseteq \mathfrak{B}_{i_{\epsilon(m)}}$, where the last inclusion is by the definition of $E$, recalling that $\overline{\mathfrak{B}}$ is increasing. Thus, $\mathfrak{B}_{i_{\epsilon(m)}} \models \mathbf{d}^{\prime}=\bigcap_{\ell<m} \mathbf{a}_{\epsilon(\ell)}^{\delta} \cap \mathbf{d}>0$, and at the same time (recall the phrase after "For $(\star)_{m+1}$ ") we have $\mathfrak{B}_{i_{\epsilon(m)}} \models 0<\mathbf{d}^{\prime} \leq \mathbf{c}_{\epsilon(m)}$. Moreover, since $\mathbf{b}_{\epsilon(m)} \in \mathfrak{B}_{i_{\epsilon(m)}}$, we have $\mathfrak{B}_{i_{\epsilon(m)}} \models 0<\mathbf{d}^{\prime} \leq \mathbf{c}_{\epsilon(m)} \leq \mathbf{b}_{\epsilon(m)}$. Then by equation (b), $\mathfrak{B}_{i_{\zeta(\epsilon(m))}} \models 0<\mathbf{d}^{\prime} \cap \mathbf{a}_{\epsilon(m)}^{\delta}$, and thus $\mathfrak{B}_{\delta} \models 0<\mathbf{d}^{\prime} \cap \mathbf{a}_{\epsilon(m)}^{\delta}$. But then the choice of $\mathbf{d}^{\prime}$ implies that $\mathfrak{B}_{\delta} \models \bigcap_{\ell \leq m} \mathbf{a}_{\epsilon(m)}^{\delta} \cap \mathbf{d}>0$, proving $(\star)_{m+1}$. This proves (d).

Clearly, this allows us to transfer the $(\kappa, \mathcal{I}, \bar{m})$-c.c. from $\mathfrak{B}_{i_{\rho_{*}}}$ to $\mathfrak{B}_{\delta}$, and it also tells us that $\left(\mathfrak{B}_{i_{\rho_{*}}}, \mathfrak{B}_{\delta}\right)$ has the $(\kappa, \mathcal{I}, \bar{m})$-pattern transfer property. By construction $\gamma_{*}<i_{\rho_{*}}$, and so by hypothesis $\left(\mathfrak{B}_{\gamma_{*}}, \mathfrak{B}_{i_{\rho_{*}}}\right)$ has the $(\kappa, \mathcal{I}, \bar{m})$-pattern transfer property, so recalling $8.14\left(\mathfrak{B}_{\gamma_{*}}, \mathfrak{B}_{\delta}\right)$ has the $(\kappa, \mathcal{I}, \bar{m})$-pattern transfer property too, which completes the proof. 
Lemma 8.19. If $\left\langle\mathfrak{B}_{\gamma}: \gamma \leq \delta\right\rangle$ is «-increasing continuous and $\mathfrak{B}_{0}$ satisfies the $(\kappa, \mathcal{I}, \bar{m})$-c.c. and $\left(\mathfrak{B}_{\gamma}, \mathfrak{B}_{\gamma+1}\right)$ satisfies the $(\kappa, \mathcal{I}, \bar{m})$-pattern transfer property for all $\gamma<\delta$, then $\mathfrak{B}_{\delta}$ satisfies the $(\kappa, \mathcal{I}, \bar{m})$-c.c. and for all $\gamma<\delta$, the pair $\left(\mathfrak{B}_{\gamma}, \mathfrak{B}_{\delta}\right)$ satisfies the $(\kappa, \mathcal{I}, \bar{m})$-pattern transfer property.

Proof. By induction on $\delta$; immediate from the previous claim and 8.14, 8.15,

We shall use the property from the proof of 8.18 later on so we phrase it below. It is stronger than 8.12, so does not supercede that definition, but as we have seen it will imply it, and occasionally it will be simpler to show.

Corollary 8.20. $\mathfrak{B}_{2}$ satisfies the $(\kappa, \mathcal{I}, \bar{m})$-c.c., and even the pair $\left(\mathfrak{B}_{1}, \mathfrak{B}_{2}\right)$ satisfies the $(\kappa, \mathcal{I}, \bar{m})$-pattern transfer property, when:

(a) $\mathfrak{B}_{1} \lessdot \mathfrak{B}_{2}$ are complete Boolean algebras

(b) $\mathfrak{B}_{1}$ satisfies the $(\kappa, \mathcal{I}, \bar{m})$-c.c.

(c) given $\mathbf{a}_{\alpha} \in \mathfrak{B}_{2}^{+}$for $\alpha<\kappa$, we can find $\mathcal{U} \in[\kappa]^{\kappa}$ and $\mathbf{x}_{\alpha} \in \mathfrak{B}_{1}^{+}$for $\alpha \in \mathcal{U}$ such that: if $u \in[\mathcal{U}]^{<\aleph_{0}}$ and $\mathfrak{B}_{1} \models$ " $\bigcap_{\alpha \in u} \mathbf{x}_{\alpha}>0$ " then $\mathfrak{B}_{2} \models$ " $\bigcap_{\alpha \in u} \mathbf{a}_{\alpha}>0$."

Remark 8.21. Note that 8.20 is superficial: we require no a priori connection between the $\mathbf{a}_{\alpha}$ 's and the $\mathbf{x}_{\alpha}$ 's other than a common enumeration. Occasionally, as for the random graph, this is enough. Still, it would be natural to add $\mathbf{x}_{\alpha} \leq_{\operatorname{proj}} \mathbf{a}_{\alpha}$.

\section{The C.C. And OMitTing Types}

The main work of this section (Lemma 9.4) is to directly connect the chain condition from 8.2 to omitting types.

Convention 9.1. In this section we fix:

(1) $\mu, \kappa, \lambda$ infinite cardinals, with $\kappa$ regular, and $\mu<\kappa \leq \lambda$.

(2) $\mathfrak{B}_{*}=\mathfrak{B}_{\kappa, \mu, \aleph_{0}}^{1}$.

(3) $\bar{m}$ a fast sequence.

(4) $\bar{E}$ a sequence of graphs which is good for $\bar{m}$.

(5) $\mathcal{I}$ an ideal on $\omega$ extending $[\omega]^{<\aleph_{0}}$.

Discussion 9.2. In terms of the three cardinals in 9.1(1): $\mu$ is the size of maximal antichains (partitions) in the completion of the free Boolean algebra $\mathfrak{B}_{*}$ we shall study in this section and use to begin our inductive construction in the next section; $\kappa$ is the cardinal in our chain condition 8.2. The cardinal $\lambda$ will ultimately be the level of saturation we are aiming for: the ultrafilter we build in later sections will realize all types over sets of size $\leq \lambda$ for theories it can handle. Lemma 9.4 will say something a priori stronger regarding non-saturation, however, since it will tell us that in certain theories, we already omit types over sets of size $\kappa$.

Discussion 9.3. Although we allow the generality of $\mu<\kappa$ with no constraints on their distance, for our main results on incomparability it would suffice to use $\mu=\aleph_{0}$ and $\kappa=\lambda$ an uncountable successor, e.g. $\aleph_{1}$. In other words, to see the differences between these theories we do not need to go out very far; however, it is not a phenomenon limited to small sizes, due to the freedom in the construction.

Historical note: the prototype for this lemma is 28 . Lemma 3.2, which amounts to showing non-saturation in our base case, for $\mathfrak{B}=\mathfrak{B}_{*}$ and any nontrivial ultrafilter $\mathcal{D}_{*}$ on $\mathfrak{B}$. We will use notation like $\mathbf{a}[\psi[\bar{a}]]$ from 7.8 above. 
Lemma 9.4. Suppose $\mathfrak{B}$ is a complete Boolean algebra, $\mathfrak{B}_{*} \lessdot \mathfrak{B}$, and $\left(\mathfrak{B}_{*}, \mathfrak{B}\right)$ has the $(\kappa, \mathcal{I}, \bar{m})$-pattern transfer property. Let $\xi$ be any level function such that $\xi^{-1}\{1\} \neq \emptyset \bmod \mathcal{I}$. Let $T=T_{\mathfrak{n}}$ where $\mathfrak{n}=\operatorname{par}[\bar{m}, \bar{E}, \xi]$. Let $\mathcal{D}$ be any ultrafilter on $\mathfrak{B}$. Then there is a possibility pattern $\left\langle\mathbf{c}_{u}: u \in[\kappa]^{<\aleph_{0}}\right\rangle$ for $T_{\mathfrak{n}}$ which has no multiplicative refinement.

Remark 9.5. So, recalling 7.9, given any $M \models T_{\mathfrak{n}}$ and any enveloping ultrapower $N=M^{\lambda} / \mathcal{D}$ for $\mathcal{D}$ and $\mathfrak{B}, N$ omits a type of $T_{\mathfrak{n}}$ over a set of size $\kappa$.

Remark 9.6. The proof of 9.4 below will show that if we fix any nonprincipal ultrafilter $\mathcal{D}_{*}$ on $\mathfrak{B}_{*}$ in advance, then for any such $T_{\mathfrak{n}}$, there is a specific possibility pattern in $\left(\mathfrak{B}_{*}, \mathcal{D}_{*}\right)$ [that is, in the initial Boolean algebra!] which fails to have a multiplicative refinement in $(\mathfrak{B}, \mathcal{D})$ for any later $\mathfrak{B}$ satisfying the hypotheses of the claim, and any ultrafilter $\mathcal{D}$ on $\mathfrak{B}$ which extends $\mathcal{D}_{*}$.

Proof of Lemma 9.4. Using the framework of separation of variables, we work in $T_{\mathfrak{n}}$, recalling that

$$
\mathfrak{n}=\operatorname{par}\left[\bar{m}, \bar{E}, \xi_{\mathfrak{n}}\right] .
$$

and recalling that

$$
\bar{m}=\left\langle m_{k}: k<\omega\right\rangle .
$$

For each $\rho \in \mathcal{T}_{2, k}$ and $\alpha<\kappa$, we define $\mathbf{a}\left[P_{\rho}\left(x_{\alpha}\right)\right] \in \mathfrak{B}_{*}^{+}$by induction on $k<\omega$ :

- if $k=0$, let $\mathbf{a}\left[P_{\langle\rangle}\left(x_{\alpha}\right)\right]=1_{\mathfrak{B}_{*}}$.

- for $k>0$, recalling $\alpha$ is also given, we first specify a finite partition of $1_{\mathfrak{B}_{*}}$ into $m_{k}$ pieces $\left\langle\mathbf{x}_{\alpha, k, i}: i<m_{k}\right\rangle$ such that every element but one of this sequence is a member of the maximal antichain $\left\{\mathbf{x}_{(\omega \alpha+k, j)}: j<\mu\right\}$ (so necessarily the remaining element is the union of the remaining members of the antichain). So without loss of generality, we may assume $\mathbf{x}_{\alpha, k, 0} \in \mathcal{D}_{*}$. Then define: for $i<m_{k}$,

$$
\mathbf{a}\left[P_{\rho-\langle i\rangle}\left(x_{\alpha}\right)\right]=\mathbf{a}\left[P_{\rho}\left(x_{\alpha}\right)\right] \cap \mathbf{x}_{\alpha, k, i} .
$$

- for $\alpha<\beta<\kappa$,

$$
\mathbf{a}\left[x_{\alpha} \neq x_{\beta}\right]=1_{\mathfrak{B}_{*}} .
$$

- Note that $\left\langle\mathbf{a}\left[P_{\rho}\left(x_{\alpha}\right)\right]: \rho \in \mathcal{T}_{2, k}\right\rangle$ is a partition of $1_{\mathfrak{B}}$.

As $\mathbf{x}_{\alpha, k, 0} \in \mathcal{D}_{*}$, letting $\left\langle 0_{k}\right\rangle$ denote the constant 0 sequence of length $k$, we have that for each $\alpha<\kappa$ and each $k<\omega$,

$$
\mathbf{a}\left[P_{\left\langle 0_{k}\right\rangle}\left(x_{\alpha}\right)\right] \in \mathcal{D}_{*} .
$$

For each $u \in[\kappa]^{<\aleph_{0}}$, define

$$
\mathbf{c}_{u}=\mathbf{a}\left[\exists x \bigwedge_{\alpha \in u} R\left(x, x_{\alpha}\right)\right] .
$$

It follows from the construction that

$$
\overline{\mathbf{c}}=\overline{\mathbf{c}}[\mathfrak{n}]=\left\langle\mathbf{c}_{u}: u \in[\kappa]^{<\aleph_{0}}\right\rangle=\left\langle\mathbf{c}_{\mathfrak{n}, u}: u \in[\kappa]^{<\aleph_{0}}\right\rangle
$$

is a possibility pattern in $\left(\mathfrak{B}_{*}, \mathcal{D}_{*}\right)$, hence in $(\mathfrak{B}, \mathcal{D})$ [thus, we could choose appropriate parameters $c_{\alpha}$ to fill in for $x_{\alpha}$ in any enveloping ultrapower].

Note to the reader: if we had run this construction for any other $\mathfrak{n}^{\prime} \in \mathbf{M}_{*}$, the elements $\mathbf{c}_{\{\alpha\}}$ would be exactly the same (and equal to $1_{\mathfrak{B}}$ ); but the sets $\mathbf{c}_{u}$ for 
$|u|>1$ could differ depending on $\mathfrak{n}^{\prime}$. So although this construction will work for any parameter in $\mathbf{M}_{*}$, it does not necessarily give the same $\overline{\mathbf{c}}$.

Assume for a contradiction that $\overline{\mathbf{a}}^{2}=\left\langle\mathbf{a}_{\alpha}^{2}: \alpha<\kappa\right\rangle$ is a multiplicative refinement of $\overline{\mathbf{c}}$ in $\mathfrak{B}$, where $\overline{\mathbf{a}}^{2}$ is a sequence of members of $\mathfrak{B}^{+}$. We apply the definition of " $\left(\mathfrak{B}_{*}, \mathfrak{B}\right)$ satisfies the $(\kappa, \mathcal{I}, \bar{m})$-pattern transfer property," hence there is a quadruple $\left(j, \mathcal{U}_{0}, A, \overline{\mathbf{a}}^{1}\right)$ as there, noting $\overline{\mathbf{a}}^{1}$ is a sequence of elements of $\mathfrak{B}_{*}^{+}$.

Now by the choice of $\mathfrak{B}_{*}$, for each $\alpha<\kappa$ there is $f_{\alpha} \in \mathrm{FI}_{\mu, \aleph_{0}}(\kappa)$, i.e. $f_{\alpha}$ is a finite function from $\kappa$ to $\mu$, such that

$$
\mathfrak{B}_{*} \models \mathbf{x}_{f_{\alpha}} \leq \mathbf{a}_{\alpha}^{1} \text { [hence this holds also in } \mathfrak{B} \text { ]. }
$$

Since each $f_{\alpha}$ has finite domain, there is a smallest positive integer $k_{\alpha}$ such that for every $\beta \in \operatorname{dom}\left(f_{\alpha}\right)$, the remainder of $\beta \bmod \omega$ is $<k_{\alpha}$. So there is $\mathcal{U}_{1} \in\left[\mathcal{U}_{0}\right]^{\kappa}$ and $n \in \omega \backslash A$ so that $k_{\alpha}=k_{\beta}<n$ for $\alpha, \beta \in \mathcal{U}_{1}$. Without loss of generality $j<n$ and $\xi_{\mathfrak{n}}(n)=1$, possible as $\xi_{\mathfrak{n}}^{-1}\{1\} \neq \emptyset \bmod \mathcal{I}$, while $A \in \mathcal{I}$. For each $\alpha \in \mathcal{U}_{1}$, the elements $\left\{\mathbf{a}\left[P_{\nu}\left(x_{\alpha}\right)\right]: \nu \in \mathcal{T}_{2, n}\right\}$ form a finite, maximal antichain of $\mathfrak{B}_{*}$. Let us justify that for some $\mathcal{U}_{2} \in\left[\mathcal{U}_{1}\right]^{\kappa}$ and some $\nu_{*} \in \mathcal{T}_{2, n}$, for every $\alpha \in \mathcal{U}_{2}$, we may extend $f_{\alpha}$ to a possibly larger finite function $f_{\alpha}^{*}$ such that

$$
0<\mathbf{x}_{f_{\alpha}^{*}} \leq \mathbf{a}\left[P_{\nu_{*}}\left(x_{\alpha}\right)\right] \text { and moreover } \mathbf{x}_{f_{\alpha}^{*}} \leq \mathbf{x}_{f_{\alpha}} .
$$

[Why? First, for each given $\alpha$, we define $f_{\alpha}^{*} \supseteq f_{\alpha}$ so that for some $\nu_{\alpha} \in \mathcal{T}_{2, n}$, $0<\mathbf{x}_{f_{\alpha}^{*}} \leq \mathbf{a}\left[P_{\nu_{\alpha}}\left(x_{\alpha}\right)\right]$. We do this by defining $f_{\alpha}^{k}$ by induction on $k<n$. Let $f_{\alpha}^{-1}=f_{\alpha}$. For $k \geq 0$, remember our finite partition $\left\langle\mathbf{x}_{\alpha, k, i}: i<m_{i}\right\rangle$ from the beginning of the proof. We want to choose $f_{\alpha}^{k} \supseteq f_{\alpha}^{k-1}$ to remain a function and so that $\mathbf{x}_{f_{\alpha}^{k}}$ is below one of the elements of this finite partition. Remember that all but one element of this partition was of the form $\mathbf{x}_{(\omega \alpha+k, j)}$, and the remaining element, call it it the "overflow element," was the union of the remaining elements of the antichain $\left\{\mathbf{x}_{(\omega \alpha+k, j)}: j<\mu\right\}$. There are two cases. If $\mathbf{x}_{f_{\alpha}^{k-1}}$ is consistent with at least one of the elements of the partition of the form $\mathbf{x}_{(\omega \alpha+k, j)}$, then choose one and define $f_{\alpha}^{k}=f_{\alpha}^{k-1} \cup\{(\omega \alpha+k, j)\}$. If not, it must already be the case that $\mathbf{x}_{f_{\alpha}^{k-1}}$ is below the overflow element, so define $f_{\alpha}^{k}=f_{\alpha}^{k-1}$. This completes the induction. Let $f_{\alpha}^{*}:=f_{\alpha}^{n-1}$. The choice of $f_{\alpha}^{*}$ determines a unique $\nu_{\alpha} \in \mathcal{T}_{2, n}$ so that $0<\mathbf{x}_{f_{\alpha}^{*}} \leq \mathbf{a}\left[P_{\nu_{\alpha}}\left(x_{\alpha}\right)\right]$. Since, again, $\mathcal{T}_{2, n}$ is finite, we may choose $\mathcal{U}_{2}$ to be a set of size $\kappa$ on which $\nu_{\alpha}$ is constant, call it $\nu_{*}$. This completes the justification of (d).]

Note that for every $\beta \in \operatorname{dom}\left(f_{\alpha}^{*}\right)$ the remainder of $\beta \bmod \omega$ is still $<n$, and it is still the case that $\operatorname{dom}\left(f_{\alpha}^{*}\right)$ is finite.

For each $\alpha<\kappa$, let $u_{\alpha}=\operatorname{dom}\left(f_{\alpha}^{*}\right)$. By the $\Delta$-system lemma 1.12, there is some $u_{*}$ and $\mathcal{U}_{3} \in\left[\mathcal{U}_{2}\right]^{\kappa}$ such that $u_{\alpha} \cap u_{\beta}=u_{*}$ for $\alpha, \beta \in \mathcal{U}_{3}$. Since the range of each $f_{\alpha}^{*}$ is a finite subset of $\mu$ and $\mu^{+} \leq \kappa$, there is $\mathcal{U}_{4} \in\left[\mathcal{U}_{3}\right]^{\kappa}$ such that $f_{\alpha}^{*}\left\lceil u_{*}=f_{\beta}^{*}\left\lceil u_{*}\right.\right.$ for $\alpha, \beta \in \mathcal{U}_{4}$. Notice this tells us for any finitely many $\alpha_{0}, \ldots, \alpha_{n-1}$ from $\mathcal{U}_{4}$, $f=\cup_{i<n} f_{i}^{*}$ is a function thus $\mathbf{x}_{f}>0$.

To summarize, for any finite $u \subseteq \mathcal{U}_{4}$, we have that in $\mathfrak{B}_{*}$,

$$
0<\left(\bigcap_{\alpha \in u} \mathbf{x}_{f_{\alpha}^{*}}\right) \leq \bigcap_{\alpha \in u} \mathbf{a}\left[P_{\nu_{*}}\left(x_{\alpha}\right)\right] .
$$

Next, note that for every $\alpha \in \mathcal{U}_{4}$, $\operatorname{dom}\left(f_{\alpha}^{*}\right) \cap\{\omega \alpha+n\}=\emptyset$, by the remark after equation (d). It follows that by our definition of $\overline{\mathbf{c}}$, for any $\ell<m_{n}$ [recalling $\bar{m}$ from (国)] and any $\alpha \in \mathcal{U}_{4}$, we have that in $\mathfrak{B}_{*}$,

$$
\mathbf{x}_{f_{\alpha}^{*}} \cap \mathbf{a}\left[P_{\nu_{*}} \sim\langle\ell\rangle\left(x_{\alpha}\right)\right]>0 .
$$


Recall that we chose $n$ so that $\xi_{\mathfrak{n}}(n)=1$. Let $w \subseteq \mathcal{U}_{4}$ be such that $|w|=m_{n}-1$. By equation (ele),

$$
\mathbf{y}_{0}:=\bigcap_{\alpha \in w} \mathbf{x}_{f_{\alpha}^{*}}>0
$$

and also recall that for each $\alpha \in w$,

$$
\mathbf{y}_{0} \leq \mathbf{a}\left[P_{\nu_{*}}\left(x_{\alpha}\right)\right]
$$

Thus, if we enumerate $w$ as $\alpha_{0}, \ldots, \alpha_{m_{n}-1}$, then in $\mathfrak{B}_{*}$ (hence also in $\mathfrak{B}$ )

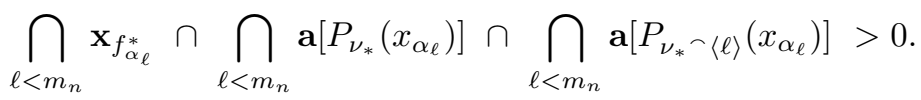

Call the quantity on the left side of this inequation " $\mathbf{y}_{1}$." Now we use the choice of the quadruple $\left(j, \mathcal{U}_{0}, A, \overline{\mathbf{a}}^{1}\right)$ for our given $w$.

In particular, we apply clause (4)(f) of 8.12 using $n=n, i=1, u=w$, and $\mathbf{a}=\mathbf{y}_{1} \in \mathfrak{B}_{*}$. Then indeed

$$
m_{n} /\left(m_{n}^{\circ}\right)<|w|<m_{n}
$$

and (g) translates to tell us that $0<\mathbf{y}_{1} \leq \bigcap_{\alpha \in w} \mathbf{a}_{\alpha}^{1}$ in $\mathfrak{B}_{*}$. So (i) of 8.12 (f) holds, and by (ii) of that clause there is $v$ such that $v \subseteq w$ and $|v| \geq|w| /\left(m_{n}^{\circ}\right)^{n^{1+j}}$ and

$$
\mathfrak{B} \models \bigcap_{\alpha \in v} \mathbf{a}_{\alpha}^{2} \cap \mathbf{y}_{1}>0 .
$$

Let us name this intersection:

$$
\mathbf{y}_{2}=\bigcap_{\alpha \in v} \mathbf{a}_{\alpha}^{2} \cap \mathbf{y}_{1} .
$$

Recall that $\overline{\mathbf{a}}^{2}$ is a solution to $\overline{\mathbf{c}}$, so by our definition of multiplicative refinement

$$
\mathfrak{B} \models \bigcap_{\alpha \in v} \mathbf{a}_{\alpha}^{2} \leq \mathbf{c}_{v} \text { which tells us that } \mathbf{y}_{2} \leq \mathbf{c}_{v} .
$$

However, since $\xi_{\mathfrak{n}}(n)=1$, the definition of a type in $T_{\mathfrak{n}}$ doesn't allow "large" splitting at $n$, so necessarily in $\mathfrak{B}$

$$
\mathbf{c}_{v} \cap\left(\bigcap_{\ell<m_{n}} \mathbf{a}\left[P_{\nu_{*}}\left(x_{\alpha_{\ell}}\right)\right] \cap \bigcap_{\ell<m_{n}} \mathbf{a}\left[P_{\nu_{*}} \sim\langle\ell\rangle\left(x_{\alpha_{\ell}}\right)\right]\right)=0 .
$$

Together (g), (h) and (ii) are a contradiction. This shows that $\overline{\mathbf{c}}$ has no multiplicative refinement.

Corollary 9.7. Recall from 8.5 that $\mathfrak{B}_{*}$ has the $(\kappa, \mathcal{I}, \bar{m})$-c.c., so by Observation 8.15, it will follow that $\mathfrak{B}$ has the $(\kappa, \mathcal{I}, \bar{m})$-c.c.

Remark 9.8. Why in the present proof do we not use the weaker choice of the pattern transfer condition 8.20 above? We will see in the next section, and in the preservation in the inductive construction.

Discussion 9.9. We could apply the first part of the proof to any $\mathfrak{m} \in \mathbf{M}_{*}$ and get a corresponding $\overline{\mathbf{a}}^{1}$, but when we fix $\mathbf{M}_{*}, \mathcal{M}, \mathcal{N}$ the cases in $\mathbf{M}_{*} \backslash \mathcal{N}$ are not useful; we needed the active level for $\mathfrak{n}$ to get a contradiction. In particular for them we will not necessarily have the failure of saturation, even though we can define the possibility pattern. 


\section{The InduCtive CONSTRUCTION}

Our construction problem naturally has two sides: we want to realize some types while omitting others.

Working in the framework of separation of variables, our strategy will be to build the Boolean algebra and the ultrafilter on it together, by induction. This is a significant advance over earlier approaches, so let us explain what makes it possible to carry it out. The rough idea will be to start with $\mathfrak{B}_{*}$ as in 9.1 (2), and at stage $\alpha$ to extend $\mathfrak{B}_{\alpha}$ and the ultrafilter on it to solve a single problem, say, a problem from a theory $T_{\mathfrak{m}}, \mathfrak{m} \in \mathcal{M}$, see 10.1(3). The work of the previous section tells us that if we can do this while maintaining " $\left(\mathfrak{B}_{*}, \mathfrak{B}_{\alpha+1}\right)$ has the $(\kappa, \mathcal{I}, \bar{m})$-pattern transfer property," we will be able to preserve non-saturation for theories $T_{\mathfrak{n}}, \mathfrak{n} \in \mathcal{N}$.

How, then, might we add solutions for problems (i.e., add multiplicative refinements for possibility patterns) while leaving our chain condition undisturbed? The idea, which seems to have much in it beyond the present proof, is that we will add the solutions in as minimal a way as possible. A Boolean algebra has generators and equation 19 they must satisfy. Roughly, given at some inductive step a possibility pattern $\left\langle\mathbf{b}_{u}: u \in[\lambda]^{<\aleph_{0}}\right\rangle$, we shall extend the given Boolean algebra by adding new elements $\left\langle\mathbf{b}_{\alpha}^{1}: \alpha \in[\lambda]^{<\aleph_{0}}\right\rangle$ to its generating set, to form the Boolean algebra generated freely by these elements, subject to any already given equations, along with the conditions saying $\mathbf{b}_{u}^{1}:=\bigcap_{\alpha \in u} \mathbf{b}_{\alpha}^{1} \leq \mathbf{b}_{u}$ which ensure $\overline{\mathbf{b}}^{1}$ is indeed a multiplicative refinement of $\mathbf{b}$. We then take any ultrafilter on this Boolean algebra which extends our previous ultrafilter and contains the elements of $\overline{\mathbf{b}}^{1}$. (See10.12.)

The proof will appeal directly to the theories involved to show that this process of adding "formal solutions" does indeed preserve our chain conditions, and the desired ultrafilters do exist. Perhaps they can be thought of as ultrafilters (and Boolean algebras) tailor-made for the theories at hand. There will be three main cases: problems coming from types over sets of size $<\kappa$ in any theory (10.20), problems coming from types over sets of size $\lambda$ in models of $T_{\mathfrak{m}}$ for $\mathfrak{m} \in \mathcal{M}$ (10.22) and problems coming from types over sets of size $\lambda$ in the random graph (10.24). Around this we set up the frame for the construction: the notion of general construction sequence, $(\theta, T)$-extension, and the normal form of (10.19).

Notice that this section establishes something quite a bit stronger than pairwise incomparable theories: we are simultaneously separating all theories $\left\{T_{\mathfrak{m}}: \mathfrak{m} \in \mathcal{M}\right\}$ from all theories $\left\{T_{\mathfrak{n}}: \mathfrak{n} \in \mathcal{N}\right\}$ in the sense of $10.1(3)$.

Convention 10.1. For this section we fix:

(1) $\bar{m}, \bar{E}, \Xi=\left\{\xi_{\alpha}: \alpha<2^{\aleph_{0}}\right\}$ satisfying the hypotheses of 6.22 ,

(2) a set $\mathbf{M}_{*}=\left\{\mathfrak{m}_{\alpha}=\operatorname{par}\left[\bar{m}, \bar{E}, \xi_{\alpha}\right]: \alpha<2^{\aleph_{0}}\right\}$ of parameters as in 6.22 ,

(3) $\mathcal{M}, \mathcal{N}$ two nonempty disjoint subsets of $\mathbf{M}_{*}$.

(4) $\aleph_{0} \leq \mu<\kappa \leq \lambda$, and $\kappa$ is regular (and uncountable). The intent of these cardinals was discussed in 9.2 above.

(5) $\mathcal{I}_{\mathcal{M}}$ the ideal corresponding to $\mathcal{M}$.

(6) $\mathfrak{B}_{*}=\mathfrak{B}_{\kappa, \mu, \aleph_{0}}^{1}$.

(7) $\mathcal{D}_{*}$ an arbitrary but fixed nonprincipal ultrafilter on $\mathfrak{B}_{*}$.

Definition 10.2. Let $\mathrm{AP}_{0}$ be the class of objects $\mathfrak{a}=\left(\mathfrak{B}_{\mathfrak{a}}, \mathcal{D}_{\mathfrak{a}}\right)$ where:

\footnotetext{
${ }^{19}$ By "equations" we mean "conditions," which can include inequalities. Also, $\mathbf{b}_{\alpha}^{1} \equiv \mathbf{b}_{\{\alpha\}}^{1}$.
} 
(1) $\mathfrak{B}_{\mathfrak{a}}$ is a complete Boolean algebra and $\mathfrak{B}_{*} \lessdot \mathfrak{B}_{\mathfrak{a}}$ (note that this is satisfied by $\mathfrak{B}_{*}$ itself).

(2) $\mathcal{D}_{\mathfrak{a}}$ is an ultrafilter on $\mathfrak{B}_{\mathfrak{a}}$ extending $\mathcal{D}_{*}$.

Definition 10.3. Let $\mathrm{AP}$ be the class of objects $\mathfrak{a}=\left(\mathfrak{B}_{\mathfrak{a}}, \mathcal{D}_{\mathfrak{a}}\right) \in \mathrm{AP}_{0}$ such that in addition $\mathfrak{B}_{\mathfrak{a}}$ satisfies the $(\kappa, \mathcal{I}, \bar{m})$-c.c.

Convention 10.4. For this section, let $\mathfrak{a}_{*}$ denote $\left(\mathfrak{B}_{*}, \mathcal{D}_{*}\right)$ from 10.1 ,

Remark 10.5. By definition $\mathfrak{a}_{*} \in \mathrm{AP}_{0}$, and by 8.5, $\mathfrak{a}_{*} \in \mathrm{AP}$.

\section{Definition 10.6.}

(1) We define a partial order on the elements of $\mathrm{AP}_{0}$ :

$$
\mathfrak{a} \leq \mathrm{AP}_{0} \mathfrak{b}
$$

when $\mathfrak{B}_{\mathfrak{a}} \lessdot \mathfrak{B}_{\mathfrak{b}}$ and $\mathcal{D}_{\mathfrak{a}} \subseteq \mathcal{D}_{\mathfrak{b}}$.

(2) $\leq_{\mathrm{AP}}$ is the following partial order on $\mathrm{AP}$ :

$$
\mathfrak{a} \leq_{\mathrm{AP}} \mathfrak{b}
$$

if and only if:

(a) $\mathfrak{a} \leq \mathrm{AP}_{0} \mathfrak{b}$

(b) $\mathfrak{a}, \mathfrak{b} \in \mathrm{AP}$

(c) the pair $\left(\mathfrak{B}_{\mathfrak{a}}, \mathfrak{B}_{\mathfrak{b}}\right)$ satisfies the $(\kappa, \mathcal{I}, \bar{m})$-pattern transfer property.

Discussion 10.7. This is a partial order by 8.14 (transitivity for pattern transfer). Recall that to show $\mathfrak{a} \leq_{\text {AP }} \mathfrak{b}$, by 8.15 it suffices to verify that $\mathfrak{a}$ has the $(\kappa, \mathcal{I}, \bar{m})$-c.c. (i.e., $\mathfrak{a} \in \mathrm{AP})$ and $\left(\mathfrak{B}_{\mathfrak{a}}, \mathfrak{B}_{\mathfrak{b}}\right)$ has the $(\kappa, \mathcal{I}, \bar{m})$-pattern transfer property.

Definition 10.8. Call $\overline{\mathfrak{b}}=\left\langle\mathfrak{b}_{\gamma}: \gamma\left\langle\gamma_{*}\right\rangle\right.$ a general construction sequence when:

(A) $\mathfrak{b}_{0}=\mathfrak{a}_{*}$, so $\mathfrak{b}_{0} \in \mathrm{AP}$.

(B) for $\gamma<\gamma_{*}, \mathfrak{b}_{\gamma} \in \mathrm{AP}_{0}$.

(C) for $\gamma<\gamma_{*}, \mathfrak{b}_{\gamma} \leq_{\mathrm{AP}_{0}} \mathfrak{b}_{\gamma+1}$.

(D) for $\gamma$ a nonzero limit ordinal, $\bigcup_{\beta<\gamma} \mathfrak{B}_{\mathfrak{b}_{\beta}}$ is a dense subset of $\mathfrak{B}_{\mathfrak{b}_{\gamma}}$ and $\mathcal{D}_{\mathfrak{b}_{\gamma}}$ is an ultrafilter on $\mathfrak{B}_{\mathfrak{b}_{\gamma}}$ which includes $\bigcup_{\beta<\gamma} \mathcal{D}_{\mathfrak{b}_{\beta}}$.

We say the length of $\overline{\mathfrak{b}}$ is $\gamma_{*}$.

This definition is justified by:

Claim 10.9. Suppose $\overline{\mathfrak{b}}=\left\langle\mathfrak{b}_{\gamma}: \gamma \leq \gamma_{*}\right\rangle$ satisfies $10.8(A)+(C)+(D)$. Then for each $\gamma \leq \gamma_{*}$, the ultrafilter $\mathcal{D}_{\mathfrak{b}_{\gamma}}$ exists, and for each $\beta<\gamma \leq \gamma_{*}, \mathfrak{B}_{\mathfrak{b}_{\beta}} \lessdot \mathfrak{B}_{\mathfrak{b}_{\gamma}}$. In particular, each $\mathfrak{b}_{\gamma} \in \mathrm{AP}_{0}$, and for each $\beta<\gamma \leq \gamma_{*}$,

$$
\mathfrak{b}_{\beta} \leq \mathrm{AP}_{0} \mathfrak{b}_{\gamma} .
$$

Proof. Let us prove, by induction on $\gamma \leq \gamma_{*}$, that each $\mathfrak{b}_{\gamma} \in \mathrm{AP}_{0}$ and that $\delta<\gamma$ implies $\mathfrak{b}_{\delta} \leq \mathrm{AP}_{0} \mathfrak{b}_{\gamma}$. For the base case, we know $\mathfrak{b}_{0} \in \mathrm{AP}_{0}$, indeed $\mathfrak{b}_{0} \in A P$. For the successor case, apply 10.8 (C), which implies membership in $\mathrm{AP}_{0}$.

Suppose we are at a limit ordinal.

For the ultrafilter: it suffices to check that for limit $\gamma, \bigcup_{\beta<\gamma} \mathcal{D}_{\mathfrak{b}_{\beta}}$ has the finite intersection property, which follows from the fact that each $\mathcal{D}_{\mathfrak{b}_{\beta}}$ is itself a filter.

For the Boolean algebras: suppose $\beta<\gamma \leq \gamma_{*}$ and $\gamma=\beta+\alpha$ and argue by induction on $\alpha$. If $\alpha=0$ this is immediate, if $\alpha$ is a successor also clear. Suppose $\alpha$ is a limit and let $X \subseteq \mathfrak{B}_{\beta}$. Let $\mathbf{a}_{\beta}$ be the supremum of $X$ in $\mathfrak{B}_{\mathfrak{b}_{\beta}}$ and let $\mathbf{a}_{\gamma}$ be the supremum of $X$ in $\mathfrak{B}_{\mathfrak{b}_{\gamma}}$. Suppose for a contradiction that in $\mathfrak{B}_{\mathfrak{b}_{\gamma}}, \mathbf{a}_{\beta} \backslash \mathbf{a}_{\gamma}=\mathbf{c}>0$. 
By definition of general construction sequence, $\bigcup_{\beta<\gamma} \mathfrak{B}_{\mathfrak{b}_{\beta}}$ is dense in $\mathfrak{B}_{\mathfrak{b}_{\gamma}}$, so there is $\delta<\gamma$ and $\mathbf{c}_{\delta} \in \mathfrak{B}_{\mathfrak{b}_{\delta}}^{+}$such that $\mathbf{c}_{\delta}<\mathbf{c}$. Then in $\mathfrak{B}_{\mathfrak{b}_{\delta}},\left(\mathbf{a}_{\beta} \backslash \mathbf{c}_{\delta}\right) \geq \mathbf{x}$ for all $\mathbf{x} \in X$, contradicting the inductive hypothesis.

Corollary 10.10. Suppose $\overline{\mathfrak{b}}=\left\langle\mathfrak{b}_{\gamma}: \gamma \leq \gamma_{*}\right\rangle$ is a general construction sequence. Suppose that for every $\beta<\gamma_{*}$, the pair $\left(\mathfrak{B}_{\mathfrak{a}_{\beta}}, \mathfrak{B}_{\mathfrak{a}_{\beta+1}}\right)$ satisfies the $(\kappa, \mathcal{I}, \bar{m})$-pattern transfer property. Then each $\mathfrak{b}_{\gamma} \in \mathrm{AP}$, and indeed for every $\beta<\delta \leq \delta_{*}$,

$$
\mathfrak{b}_{\beta} \leq_{\mathrm{AP}} \mathfrak{b}_{\delta}
$$

Proof. By Claim 8.5, Lemma 8.19, and Claim 10.9,

Remark 10.11. Note to the reader: sometimes $\theta$ has a special meaning in Boolean algebras, such as an upper bound on the intersections allowed in a free Boolean algebra under consideration. In the present paper, that role is always played by $\aleph_{0}$, so $\theta$ is free to be used for other things, as in the next definition.

Our next definition expresses that we extend $\left(\mathfrak{B}_{\mathfrak{a}}, \mathcal{D}_{\mathfrak{a}}\right)$ in a certain minimal way: by simply adding a formal solution to some possibility pattern $\overline{\mathbf{b}}=\left\langle\mathbf{b}_{u}: u \in[\theta]^{\left\langle\aleph_{0}\right.}\right\rangle$ for some theory. For now, the definition is general, allowing the size $\theta$ and the theory to vary. We could think about such extensions as simply ensuring an instance of goodness, adding some multiplicative refinement to some monotonic function. The crucial point is that we do this as freely as possible, essentially only requiring that the equations in $\mathfrak{B}_{\mathfrak{a}}$ are still respected, and the new addition $\left\langle\mathbf{b}_{\{\alpha\}}^{1}: \alpha<\theta\right\rangle$ is a formal solution to $\overline{\mathbf{b}}$, i.e., for each $u \in[\theta]^{<\aleph_{0}}, \bigcap_{\alpha \in u} \mathbf{b}_{\alpha}^{1} \leq \mathbf{b}_{u}$. Since we are adding multiplicative refinements, it suffices to specify $\mathbf{b}_{u}^{1}$ for $|u|=1$. As noted there, in the rest of the paper, we will often drop parentheses when $|u|=1$, writing $\mathbf{b}_{\alpha}^{1}$ instead of $\mathbf{b}_{\{\alpha\}}^{1}$. Notice that by 10.12(3), we will need to check existence.

Definition 10.12. Suppose $\mathfrak{a} \in \mathrm{AP}_{0}, T$ is a complete first-order theory, and $\theta \leq \lambda$ is an infinite cardinal. Say that $\mathfrak{b}=\left(\mathfrak{B}_{\mathfrak{b}}, \mathcal{D}_{\mathfrak{b}}\right)$ is a $(\theta, T)$-extension of $\mathfrak{a}$ when there exists a possibility pattern $\overline{\mathbf{b}}=\left\{\mathbf{b}_{u}: u \in[\theta]<\aleph^{<}\right\}$in $\left(\mathfrak{B}_{\mathfrak{a}}, \mathcal{D}_{\mathfrak{a}}\right)$ for the theory $T$ and:

(1) $\mathfrak{B}_{\mathfrak{b}}$ is the completion of the Boolean algebra $\mathfrak{B}$ generated by the set $\mathcal{Y}_{\mathfrak{a}, \mathfrak{b}}$ which is $\mathfrak{B}_{\mathfrak{a}}$ along with the set of new elements $\left\{\mathbf{b}_{\{\alpha\}}^{1}: \alpha<\theta\right\}$, freely except for the set of equations $\Gamma_{\mathfrak{a}, \mathfrak{b}}$ which are 20

(a) the equations already in $\mathfrak{B}_{\mathfrak{a}}$.

(b) for every nonempty finite $u \subseteq \theta$,

$$
\bigcap_{\alpha \in u} \mathbf{b}_{\{\alpha\}}^{1} \leq \mathbf{b}_{u} .
$$

(2) Notation: for $|u|>1$, let $\mathbf{b}_{u}^{1}:=\bigcap_{\alpha \in u} \mathbf{b}_{\{\alpha\}}^{1}$. Convention: $\mathbf{b}_{\emptyset}^{1}=1_{\mathfrak{B}}$.

(3) Convention: for readability, when $u=\{\alpha\}$ and it is unlikely to cause confusion, we may drop parentheses and write $\mathbf{b}_{\alpha}^{1}$ for $\mathbf{b}_{\{\alpha\}}^{1}$, so the new elements are $\left\{\mathbf{b}_{\alpha}^{1}: \alpha<\theta\right\}$.

(4) $\mathcal{D}_{\mathfrak{b}}$ is an ultrafilter on $\mathfrak{B}_{\mathfrak{b}}$ which agrees with $\mathcal{D}_{\mathfrak{a}}$ on $\mathfrak{B}_{\mathfrak{a}}$, and such that $\mathbf{b}_{\alpha}^{1} \in \mathcal{D}_{\mathfrak{b}}$ for all $\alpha<\theta$, if such an ultrafilter exists; otherwise not defined.

We may say $\mathfrak{b}$ is an $(\theta, T, \overline{\mathbf{b}})$-extension of $\mathfrak{a}$ to emphasize that $\overline{\mathbf{b}}$ is the possibility pattern acquiring a solution.

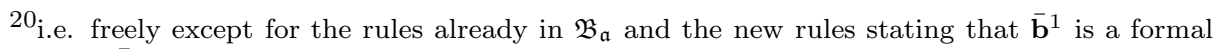
solution to $\overline{\mathbf{b}}$.
} 
Remark 10.13. Recalling 7.8, a possibility pattern for $T$ comes naturally equipped with the data of $\mathbf{a}\left[\psi\left[\bar{x}_{u}\right]\right] \in \mathfrak{B}_{\mathfrak{a}}$ for $\psi \in \mathcal{L}\left(\tau_{T}\right)$; we will use these in some proofs.

We record the following here though it refers to upcoming proofs:

Observation 10.14. If $\mathfrak{b} \in \mathrm{AP}$ is a $(\theta, T, \overline{\mathbf{b}})$-extension of $\mathfrak{a} \in \mathrm{AP}$, and:

(1) $\theta<\kappa$, or

(2) $T=T_{\mathfrak{m}}$ for $\mathfrak{m} \in \mathcal{M}$ and $\overline{\mathbf{b}}$ is a possibility pattern coming from a positive $P_{\nu}(x) \wedge R(x, y)$-type for some $\nu \in \mathcal{T}_{1}$, or

(3) $T=T_{\mathbf{r g}}$ and $\overline{\mathbf{b}}$ is a possibility pattern coming from a type in positive and negative instances of the graph edge relation,

then

$$
\left|\mathfrak{B}_{\mathfrak{b}}\right| \leq\left(\left|\mathfrak{B}_{\mathfrak{a}}\right|+\lambda\right)^{\kappa}
$$

Proof. By the $\kappa$-c.c. which follows from " $\mathfrak{a}, \mathfrak{b} \in$ AP", and which is proved in Claim 10.20 for (1), Theorem 10.22 for (2) and Lemma 10.24 for (3). Alternately, we could use $\lambda \geq \theta, \kappa$ and conclude $\left|\mathfrak{B}_{\mathfrak{b}}\right| \leq\left(\left|\mathfrak{B}_{\mathfrak{a}}\right|+\lambda\right)^{\lambda}$, which suffices for our purposes.

Corollary 10.15. Suppose $\left\langle\mathfrak{b}_{\gamma}: \gamma \leq \gamma_{*}\right\rangle$ is a general construction sequence with $\gamma_{*} \leq 2^{\lambda}$, and for each $\gamma<\gamma_{*}, \mathfrak{b}_{\gamma+1}$ is a $(\theta, T, \overline{\mathbf{b}})$-extension of $\mathfrak{b}_{\gamma}$ in the sense of 10.14(1), (2) or (3). Then $\left|\mathfrak{B}_{\mathfrak{b}_{\gamma}}\right| \leq 2^{\lambda}$ for all $\gamma \leq \gamma_{*}$.

Proof. By induction on $\gamma$. For $\gamma=0,\left|\mathfrak{B}_{*}\right| \leq 2^{\lambda}$; for $\gamma=\beta+1$, apply 10.14 and at nonzero limit stages, use the $\kappa$-c.c. and the fact that $|\gamma| \leq 2^{\lambda}$.

Remark 10.16. In the definition we put no restrictions on the theory; only in the actual construction do we use $T_{\mathfrak{n}}, T_{\mathfrak{m}}$ and $T_{\mathbf{r g}}$.

Claim 10.17. Suppose $\mathfrak{b}$ is a $(\theta, T, \overline{\mathbf{b}})$-extension of $\mathfrak{a} \in \mathrm{AP}$, for some theory $T$ and some $\theta \leq \lambda$. Then:

(1) $\mathfrak{B}_{\mathfrak{a}} \subseteq \mathfrak{B}_{\mathfrak{b}}$,

(2) indeed, $\mathfrak{B}_{\mathfrak{a}} \lessdot \mathfrak{B}_{\mathfrak{b}}$.

(3) there exists an ultrafilter $\mathcal{D}$ on $\mathfrak{B}_{\mathfrak{b}}$ which agrees with $\mathcal{D}_{\mathfrak{a}}$ on $\mathfrak{B}_{\mathfrak{a}}$ and contains $\mathbf{b}_{\alpha}^{1}$ for all $\alpha<\theta$, hence $\mathcal{D}_{\mathfrak{b}}$ is such an ultrafilter.

Proof. Recall from 10.12 that $\mathfrak{B}_{\mathfrak{b}}$ is the completion of the Boolean algebra generated by $\mathcal{Y}=\mathfrak{B}_{\mathfrak{a}} \cup\left\{\mathbf{b}_{\alpha}^{1}: \alpha<\theta\right\}$ freely except for the set of equations $\Gamma$, which include all equations already in $\mathfrak{B}_{\mathfrak{a}}$ along with equations saying that for each finite $u \in[\theta]^{<\aleph_{0}}$, $\bigcap_{\alpha \in u} \mathbf{b}_{\alpha}^{1} \leq \mathbf{b}_{u}$.

For each $u \in[\theta]<\aleph_{0}$, define $h_{u}: \mathcal{Y} \rightarrow \mathfrak{B}_{\mathfrak{a}}$ as follows: $h_{u}\left\lceil\mathfrak{B}_{\mathfrak{a}}\right.$ is the identity, $h_{u}\left(\mathbf{b}_{\alpha}^{1}\right)=\mathbf{b}_{u}$ if $\alpha \in u$, and $h_{u}\left(\mathbf{b}_{\alpha}^{1}\right)=0_{\mathfrak{B}_{\mathfrak{a}}}$ if $\alpha \in \theta \backslash u$. Note that $h_{u}$ respects the equations in $\Gamma$.

To see that $\mathfrak{B}_{\mathfrak{a}} \subseteq \mathfrak{B}_{\mathfrak{b}}$, note that in the case $u=\emptyset$ (as the generators are dense in the completion) the map $h_{\emptyset}$ induces an endomorphism $\hat{h}_{\emptyset}$ from $\mathfrak{B}_{\mathfrak{b}}$ onto $\mathfrak{B}_{\mathfrak{a}}$ which extends the identity map on $\mathfrak{B}_{\mathfrak{a}}$. This proves (a).

Next we work towards showing that $\mathfrak{B}_{\mathfrak{a}}$ is a complete subalgebra of $\mathfrak{B}_{\mathfrak{b}}$. Note that $\mathfrak{B}_{\mathfrak{b}}$ is by definition a complete Boolean algebra. Fix for awhile $\mathbf{c} \in \mathfrak{B}_{\mathfrak{b}}^{+}$. As the generators are dense in the completion, we may find

$$
\mathbf{x} \in \mathfrak{B}_{\mathfrak{a}}^{+}, u, u_{0}, \ldots, u_{n-1} \in[\theta]^{<\aleph_{0}}
$$


such that $u_{\ell} \nsubseteq u$ for $\ell<n$ and

$$
\mathfrak{B}_{\mathfrak{b}} \models 0<\mathbf{x} \cap \mathbf{b}_{u}^{1} \cap \bigcap_{\ell<n}\left(-\mathbf{b}_{u_{\ell}}^{1}\right) \leq \mathbf{c} .
$$

[Note that $\left\{\mathbf{b}_{\alpha}^{1}: \alpha<\theta\right\}$ generates a multiplicative sequence: $\mathbf{b}_{u}^{1}=\bigcap_{\alpha \in u} \mathbf{b}_{\alpha}^{1}$ for any finite $u \subseteq \theta$. So the positive intersection in the inset equation may be given by a single $u$. We could have taken the $u_{\ell}$ 's to be singletons, without loss of generality. Note that we could also have assumed, without loss of generality, that $\mathbf{x} \leq \mathbf{b}_{u}$.]

Thus, for $\mathbf{c}, \mathbf{x}, u, u_{0}, \ldots, u_{n-1}$ as in the previous paragraph, the map $\hat{h}_{u}$ is constant on $\mathfrak{B}_{\mathfrak{a}}$, takes $\mathbf{b}_{\alpha}^{1}$ to $\mathbf{b}_{u}$ for $\alpha \in u$, and $\mathbf{b}_{\alpha}^{1}$ to $0_{\mathfrak{B}_{\mathfrak{a}}}$ for $\alpha \in \theta \backslash u$, hence takes $\mathbf{b}_{u}^{1}$ to $\mathbf{b}_{u}$, and each $\mathbf{b}_{u_{\ell}}^{1}$ to $0_{\mathfrak{B}_{\mathfrak{a}}}($ for $\ell<n)$.

It follows that for any $\mathbf{d} \in \mathfrak{B}_{\mathfrak{b}}^{+}$, if $\mathfrak{B}_{\mathfrak{a}} \models 0<\mathbf{d} \leq \mathbf{x} \cap \mathbf{b}_{u}$ then $\mathfrak{B}_{\mathfrak{b}} \models 0<\mathbf{d} \cap \mathbf{c}$ [i.e., $\mathbf{x} \cap \mathbf{b}_{u}$ is below the projection of $\mathbf{c}$, or if we chose $\mathbf{x} \leq \mathbf{b}_{u}$, that already $\mathbf{x}$ is below the projection of $\mathbf{c}$.

Since $\mathbf{c}$ was arbitrary, we have shown that any maximal antichain of $\mathfrak{B}_{\mathfrak{a}}$ will remain a maximal antichain of $\mathfrak{B}_{\mathfrak{b}}$ (if not, there is some nonzero $\mathbf{c} \in \mathfrak{B}_{\mathfrak{b}}^{+}$which does not have nonempty intersection with any element of the antichain; but its corresponding $\mathbf{x} \cap \mathbf{b}_{u}$ must, contradiction). This completes the proof of (b).

Finally, to verify (c), it suffices to show that $\mathcal{D}_{\mathfrak{a}} \cup\left\{\mathbf{b}_{\alpha}^{1}: \alpha<\theta\right\}$ has the finite intersection property in $\mathfrak{B}_{\mathfrak{b}}$, as then it can be extended to an ultrafilter. This follows from the existence of the $\hat{h}_{u}$ 's. (Suppose that for some finite $u$ and some set $\mathbf{a} \in \mathcal{D}_{\mathfrak{a}}, \mathbf{a} \cap \bigcap\left\{\mathbf{b}_{\alpha}^{1}: \alpha \in u\right\}=0_{\mathfrak{B}_{\mathfrak{b}}}$. Then $\hat{h}_{u}\left(\mathbf{b}_{\alpha}^{1}\right)=\mathbf{b}_{u}$ for each $\alpha \in u$. Recall that $\left\langle\mathbf{b}_{u}: u \in[\theta]^{<\aleph_{0}}\right\rangle$ was a possibility pattern for $\left(\mathfrak{B}_{\mathfrak{a}}, \mathcal{D}_{\mathfrak{a}}\right)$, thus a sequence of elements of $\mathcal{D}_{\mathfrak{a}}$; in particular, $\mathbf{b}_{u} \in \mathcal{D}_{\mathfrak{a}}$, so $\mathfrak{B}_{\mathfrak{a}} \models \mathbf{b}_{u} \cap \mathbf{a}>0$, contradiction.)

We record a simple variant for future proofs:

${ }^{\star}$ Observation 10.18. Suppose that $\mathbf{a} \in \mathrm{AP}$ but instead of taking a $(\theta, T)$-extension of $\mathfrak{B}_{\mathfrak{a}}$, we consider $\mathfrak{B}$ which is generated from $\mathfrak{B}_{\mathfrak{a}}$ along with up to $\theta$ new antichains each of cardinality $<\kappa$, as freely as possible: that is, for some $h \in{ }^{\theta} \kappa, \mathfrak{B}_{\mathfrak{a}} \cup\left\{\mathbf{c}_{\alpha, \epsilon}\right.$ : $\epsilon<h(\alpha), \alpha<\theta\}$ freely except for the equations already in $\mathfrak{B}_{\mathfrak{a}}$ and the equations saying that for each $\alpha,\left\{\mathbf{c}_{\alpha, \epsilon}: \epsilon<h(\alpha)\right\}$ is an antichain. Let $\mathfrak{B}_{\mathfrak{b}}$ be the completion of $\mathfrak{B}$. Then the proof that

(1) $\mathfrak{B}_{\mathfrak{a}} \subseteq \mathfrak{B}_{\mathfrak{b}}$

(2) indeed $\mathfrak{B}_{\mathfrak{a}} \lessdot \mathfrak{B}_{\mathfrak{b}}$

(3) $\mathfrak{B}_{\mathfrak{b}}$ has the $\kappa$-c.c.

is easier than in 10.17 , and just as in 10.15 , we may conclude $\left|\mathfrak{B}_{\mathfrak{b}}\right| \leq\left(\left|\mathfrak{B}_{\mathfrak{a}}\right|+\mu\right)^{<\kappa}$.

Keeping in mind 10.10, our main task now will be to show that we can preserve the pattern transfer property at successor stages realizing certain specific types for certain specific theories. We will make repeated use of the move in the proof of 10.17 equation (国), giving a useful "normal form" for elements, so we start by summarizing it here. Note in the next observation that we bound $\theta$ by $\lambda$, and in particular, it is possible for $\theta$ to be larger than $\kappa$ (recalling the remark before 1.12).

Observation 10.19. Suppose $\mathfrak{a} \in \mathrm{AP}$ and $\mathfrak{b}$ is a $(\theta, T, \overline{\mathbf{b}})$-extension of $\mathfrak{a}$ for some $\theta \leq \lambda$. Suppose we are given a sequence $\left\langle\mathbf{a}_{\alpha}^{2}: \alpha<\kappa\right\rangle$ of elements of $\mathfrak{B}_{\mathfrak{b}}^{+}$. Then:

(1) for each $\alpha<\kappa$, there is $\mathbf{i}_{\alpha}=\left(\mathbf{x}_{\alpha}, u_{\alpha},\left\langle u_{\alpha, \ell}: \ell<n_{\alpha}\right\rangle\right)$ such that $\mathbf{x}_{\alpha} \in \mathfrak{B}_{\mathfrak{a}}^{+}$; $n_{\alpha} \in \mathbb{N} ; u_{\alpha}, u_{\alpha, 0}, \ldots, u_{\alpha, n_{\alpha}-1} \in[\theta]^{<\aleph_{0}} ; u_{\alpha} \nsubseteq u_{\alpha, \ell}$ for $\ell<n_{\alpha} ; \mathbf{x}_{\alpha} \leq \mathbf{b}_{u_{\alpha}} ;$ 
KEISLER'S ORDER IS NOT SIMPLE (AND SIMPLE THEORIES MAY NOT BE EITHER) 47

and

$$
\mathfrak{B}_{\mathfrak{b}} \models 0<\mathbf{x}_{\alpha} \cap \mathbf{b}_{u_{\alpha}}^{1} \cap \bigcap_{\ell<n_{\alpha}}\left(-\mathbf{b}_{u_{\alpha, \ell}}^{1}\right) \leq \mathbf{a}_{\alpha}^{2} .
$$

[i.e., since the generators are dense in the completion, we can find a positive element below $\mathbf{a}_{\alpha}^{2}$ which is the intersection of an element from $\mathfrak{B}_{\mathfrak{a}}$, some number of new elements, and some number of negations of (intersections of) new elements.]

(2) Given $\mathbf{i}_{\alpha}$ for $\alpha<\kappa$ from (1), define $w_{\alpha}=u_{\alpha} \cup \bigcup\left\{u_{\alpha, \ell}: \ell<n_{\alpha}\right\}$. Then there are $\mathcal{U} \in[\kappa]^{\kappa}, w_{*}, u_{*}, n_{*},\left\langle u_{\ell}^{*}: \ell<n_{*}\right\rangle$ such that for every $\alpha \in \mathcal{U}$, $w_{\alpha}=w_{*}, n_{\alpha}=n_{*}, u_{\alpha} \cap w_{*}=u_{*}, u_{\alpha, \ell} \cap w_{*}=u_{\ell}^{*}$.

[i.e., by applying the $\Delta$-system lemma we can smooth this out on a large set.]

(3) For every $\alpha \in \mathcal{U}$ and $\mathbf{x}_{\alpha}$ from $\mathbf{i}_{\alpha}$, we have that $\mathbf{x}_{\alpha} \leq_{\text {proj }} \mathbf{a}_{\alpha}^{2}$.

(4) Suppose $\mathcal{U}$ is from (2) and $X \subseteq \mathcal{U}$ is finite and $\mathbf{a}_{*} \in \mathfrak{B}_{\mathfrak{a}}^{+}$. Suppose

$$
\mathfrak{B}_{\mathfrak{b}} \models \mathbf{a}_{*} \cap \bigcap_{\alpha \in X}\left(\mathbf{x}_{\alpha} \cap \mathbf{b}_{u_{\alpha}}^{1}\right)>0 .
$$

Then also

$$
\mathfrak{B}_{\mathfrak{b}} \models \mathbf{a}_{*} \cap \bigcap_{\alpha \in X}\left(\mathbf{x}_{\alpha} \cap \mathbf{b}_{u_{\alpha}}^{1} \cap \bigcap_{\ell<n_{\alpha}}\left(-\mathbf{b}_{u_{\alpha, \ell}}^{1}\right)\right)>0
$$

[i.e., when checking for positive intersections we may safely ignore complements.]

Proof. For part (1), the generators are dense in the completion, and as mentioned in the proof of 10.17, we can gather the intersection of elements of the form $\mathbf{b}_{\beta}^{1}$ into a single $\mathbf{b}_{u_{\alpha}}^{1}$. Since $\mathbf{x}_{\alpha} \cap \mathbf{b}_{u}>0$ in $\mathfrak{B}_{\mathfrak{a}}$, there is no harm in assuming $\mathfrak{B}_{\mathfrak{a}} \models \mathbf{x}_{\alpha} \leq \mathbf{b}_{u}$.

For part (2), by the $\Delta$-system lemma (recall that $\kappa$ is regular) there is $\mathcal{U} \in[\kappa]^{\kappa}$ such that $\left\langle w_{\alpha}: \alpha \in \mathcal{U}\right\rangle$ is a $\Delta$-system with heart $w_{*}$. So we can assume for some $u_{*}$, $n_{*},\left\langle u_{\ell}^{*}: \ell<n\right\rangle$, for every $\alpha \in \mathcal{U}$, we have that $n_{\alpha}=n, u_{\alpha} \cap w_{*}=u_{*}, u_{\alpha, \ell} \cap w_{*}=u_{\ell}^{*}$. Note we may ask for additional uniformity, e.g. that the $u_{\alpha}$ 's have constant size.

To verify $\mathbf{x}_{\alpha} \leq_{\text {proj }} \mathbf{a}_{\alpha}^{2}$ for (c), it suffices to show that there is an endomorphism $f$ from $\mathfrak{B}_{\mathfrak{b}}$ onto $\mathfrak{B}_{\mathfrak{a}}$ fixing $\mathfrak{B}_{\mathfrak{a}}$ pointwise such that $f\left(\mathbf{a}_{\alpha}^{2}\right) \geq \mathbf{x}_{\alpha}$. Consider the map $\hat{h}_{u_{\alpha}}: \mathfrak{B}_{\mathfrak{b}} \rightarrow \mathfrak{B}_{\mathfrak{a}}$ defined in the proof of 10.17 . Then $\hat{h}_{u_{\alpha}}\left(\mathbf{x}_{\alpha}\right)=\mathbf{x}_{\alpha}, \hat{h}_{u_{\alpha}}\left(\mathbf{b}_{u_{\alpha}}^{1}\right)=\mathbf{b}_{u_{\alpha}}$, and by the disjointness conditions of the $\Delta$-system, $\hat{h}_{u_{\alpha}}\left(\mathbf{b}_{u_{\alpha, \ell}}^{1}\right)=0_{\mathfrak{B}_{\mathfrak{a}}}$ for all $\ell<n$. So $\hat{h}_{u_{\alpha}}\left(\mathbf{a}_{\alpha}^{2}\right) \geq \mathbf{x}_{\alpha} \cap \mathbf{b}_{u_{\alpha}}=\mathbf{x}_{\alpha}$, recalling that we assumed $\mathbf{x}_{\alpha} \leq \mathbf{b}_{u_{\alpha}}$ in part (1).

Part (4) is similar. First consider the simple case where $\mathbf{a}_{*}=1_{\mathfrak{B}_{\mathfrak{a}}}$, so we may ignore it. Let

$$
u=\bigcup_{\alpha \in X} u_{\alpha} .
$$

Recall that $\mathfrak{B}_{\mathfrak{b}}^{1} \models \bigcap_{\alpha \in X} \mathbf{b}_{u_{\alpha}}^{1}=\mathbf{b}_{u}^{1}$, since $\overline{\mathbf{b}}^{1}$ is multiplicative, and $\mathbf{b}_{u}^{1} \leq \mathbf{b}_{u}$, since the sequence $\overline{\mathbf{b}}^{1}$ refines the sequence $\overline{\mathbf{b}}$. Thus in $\mathfrak{B}_{\mathfrak{b}}^{1}$, starting with our assumption,

(a) $\quad 0<\bigcap_{\alpha \in X}\left(\mathbf{x}_{\alpha} \cap \mathbf{b}_{u_{\alpha}}^{1}\right)=\bigcap_{\alpha \in X} \mathbf{x}_{\alpha} \cap \bigcap_{\alpha \in X} \mathbf{b}_{u_{\alpha}}^{1}=\bigcap_{\alpha \in X} \mathbf{x}_{\alpha} \cap \mathbf{b}_{u}^{1} \leq \bigcap_{\alpha \in X} \mathbf{x}_{\alpha} \cap \mathbf{b}_{u}$.

Let $\mathbf{y}=\bigcap_{\alpha \in X}\left(\mathbf{x}_{\alpha} \cap \mathbf{b}_{u_{\alpha}}^{1}\right)$. It suffices to show there is an endomorphism $f$ from $\mathfrak{B}_{\mathfrak{b}}$ onto $\mathfrak{B}_{\mathfrak{a}}$ such that $f(\mathbf{y})>0$ but $f\left(\mathbf{b}_{u_{\alpha, \ell}}^{1}\right)=0_{\mathfrak{B}_{\mathfrak{a}}}$ for all $\alpha \in X, \ell<n$. Recalling again the map from the proof of 10.17, $\hat{h}_{u}: \mathfrak{B}_{\mathfrak{b}} \rightarrow \mathfrak{B}_{\mathfrak{a}}$. Then $\hat{h}_{u}\left(\mathbf{x}_{\alpha}\right)=\mathbf{x}_{\alpha}$ for 
$\alpha \in X$, and $\hat{h}_{u}\left(\mathbf{b}_{\beta}^{1}\right)=\mathbf{b}_{u}$ for all $\alpha \in X$ and $\beta \in u_{\alpha}$. Thus, $\hat{h}_{u}\left(\mathbf{b}_{u_{\alpha}}^{1}\right)=\mathbf{x}_{\alpha} \cap \mathbf{b}_{u}$ for $\alpha \in X$, so remembering equation (园), $\hat{h}_{u}(\mathbf{y})=\bigcap_{\alpha \in X} \mathbf{x}_{\alpha} \cap \mathbf{b}_{u}>0$. The effect of the $\Delta$-system ensures that $u_{\alpha, \ell} \cap u=\emptyset$ for $\alpha \in X, \ell<n$, so $\hat{h}_{u}\left(\mathbf{b}_{\beta}^{1}\right)=0_{\mathfrak{B}_{\mathfrak{a}}}$ for $\beta \in u_{\alpha, \ell}$, $\alpha \in X, \ell<n$, as desired.

Now assume that $\mathbf{a}_{*} \in \mathfrak{B}_{\mathfrak{a}}^{+}$is arbitrary but given. Equation (国), condensed for space reasons, becomes:

$$
0<\mathbf{a}_{*} \cap \bigcap_{\alpha \in X}\left(\mathbf{x}_{\alpha} \cap \mathbf{b}_{u_{\alpha}}^{1}\right)=\cdots \cdots \leq \mathbf{a}_{*} \cap \bigcap_{\alpha \in X} \mathbf{x}_{\alpha} \cap \mathbf{b}_{u} .
$$

Let $\mathbf{z}=\mathbf{a}_{*} \cap \mathbf{y}$. Under the same map $\hat{h}_{u}$, note $\hat{h}_{u}\left(\mathbf{a}_{*}\right)=\mathbf{a}_{*}$ since it is an element of $\mathfrak{B}_{\mathfrak{a}}$. So by equation (b), $\hat{h}_{u}(\mathbf{z})=\mathbf{a}_{*} \cap \bigcap_{\alpha \in X} \mathbf{x}_{\alpha} \cap \mathbf{b}_{u}>0$ as desired.

Our next claim says essentially that if $\theta<\kappa$ then there is no problem realizing any $(T, \theta)$-type for any $T$. Thus, we can arrange for our final ultrafilters to be $\kappa$-good, even though they will be far from $\kappa^{+}$-good.

Claim 10.20 (Realizing small types). Assume $\mathfrak{a} \in \mathrm{AP}, \mathfrak{b}$ is a $(\theta, T, \overline{\mathbf{b}})$-extension of $\mathfrak{a}$, where $\theta<\kappa$. Then $\left(\mathfrak{B}_{\mathfrak{a}}, \mathfrak{B}_{\mathfrak{b}}\right)$ satisfies the $(\kappa, \mathcal{I}, \bar{m})$-pattern transfer property.

Proof. This proof and the proof of 10.22 share a picture, so in this simpler case, we go slowly to motivate the second proof.

Let $\left\langle\mathbf{a}_{\alpha}^{2}: \alpha<\kappa\right\rangle$ be given, with each $\mathbf{a}_{\alpha}^{2} \in \mathfrak{B}_{\mathfrak{b}}^{+}$. First we appeal to the normal form of 10.19(1): for all $\alpha<\kappa$ we can find $\mathbf{i}_{\alpha}=\left(\mathbf{x}_{\alpha}, u_{\alpha},\left\langle u_{\alpha, \ell}: \ell<n_{\alpha}\right\rangle\right)$ as there, so $\mathbf{x}_{\alpha} \leq \mathbf{b}_{u_{\alpha}}$, and

$$
\mathfrak{B}_{\mathfrak{b}}=0<\mathbf{x}_{\alpha} \cap \mathbf{b}_{u_{\alpha}}^{1} \cap \bigcap_{\ell<n_{\alpha}}\left(-\mathbf{b}_{u_{\alpha, \ell}}^{1}\right) \leq \mathbf{a}_{\alpha}^{2} .
$$

In our present case, we don't need to appeal to the $\Delta$-system reduction of $10.19(2)$, since something stronger is true: we have assumed $\theta<\kappa$, and note each $u_{\alpha}, u_{\alpha, \ell}$ is a finite subset of $\theta$. So we may find $\mathcal{U} \in[\kappa]^{\kappa}$ on which the sequence

$$
\left\langle\left(u_{\alpha},\left\langle u_{\alpha, \ell}: \ell<n_{\alpha}\right\rangle\right): \alpha \in \mathcal{U}\right\rangle
$$

is constant, and say equal to $\left(u_{\oplus},\left\langle u_{\oplus, \ell}: \ell<n_{\oplus}\right\rangle\right)$. Set

$$
\mathbf{a}_{\alpha}^{1}=\mathbf{x}_{\alpha} \cap \mathbf{b}_{u_{\oplus}}=\mathbf{x}_{\alpha} \text { for each } \alpha \in \mathcal{U} .
$$

Then each $\mathbf{a}_{\alpha}^{1} \leq_{\text {proj }} \mathbf{a}_{\alpha}^{2}$ (by 10.19(3)). Let us verify [8.12(4)(f) holds for $A=\emptyset$, $j=0$ to transfer from $\overline{\mathbf{a}}^{1}$ to $\overline{\mathbf{a}}^{2}$. Suppose we are given $n \in \omega \backslash A$, a finite $u \subseteq \mathcal{U}$, and a nonzero $\mathbf{a}_{*} \in \mathfrak{B}_{\mathfrak{a}}^{+}$such that $m_{n} /\left(m_{n}^{\circ}\right)^{n^{i}}<|u|<m_{n}$ and

$$
\mathfrak{B}_{\mathfrak{a}} \models 0<\mathbf{a}_{*} \leq \bigcap_{\alpha \in u} \mathbf{a}_{\alpha}^{1} .
$$

As $j=0$, to fulfill the $(\kappa, \mathcal{I}, \bar{m})$-pattern transfer we would like to find $v$ such that $v \subseteq u$ and $|v| \geq|u| /\left(m_{n}^{\circ}\right)^{n^{i+0}}$ and

$$
\mathfrak{B}_{2} \models \bigcap_{\alpha \in v} \mathbf{a}_{\alpha}^{2} \cap \mathbf{a}_{*}>0 .
$$

Remembering 10.19(4), to prove (d) it would suffice to show that for this $v$,

$$
\mathfrak{B}_{\mathfrak{b}} \models \bigcap_{\alpha \in v}\left(\mathbf{x}_{\alpha} \cap \mathbf{b}_{u_{\alpha}}^{1}\right) \cap \mathbf{a}_{*}>0 .
$$


Let us verify (四) holds already for $v=u$. [Clearly this choice of $v$ has an acceptable size.] Let $w=\bigcup_{\alpha \in v} u_{\alpha}$. Observe that to prove (国), it would suffice to show that 21

(f) for some nonzero $\mathbf{a}_{* *} \in \mathfrak{B}_{\mathfrak{a}}$ with $0<\mathbf{a}_{* *} \leq \mathbf{a}_{*}$, we have $\mathbf{a}_{* *} \leq \mathbf{b}_{w(v)}$.

[Why would this suffice? By the choice of $\mathbf{a}_{* *}$ and equation (ㄷ), we know that $0<\mathbf{a}_{* *} \leq \mathbf{a}_{*} \leq \bigcap_{\alpha \in v} \mathbf{x}_{\alpha}$, since by (b) $\mathbf{a}_{\alpha}^{1}$ is just another name for $\mathbf{x}_{\alpha}$. Now similarly to earlier proofs 10.17 and 10.19, $\hat{h}_{w}$ is an endomorphism from $\mathfrak{B}_{\mathfrak{b}}$ onto $\mathfrak{B}_{\mathfrak{a}}$ which is the identity on $\mathfrak{B}_{\mathfrak{a}}$ and for each $\alpha \in v$ takes $\mathbf{x}_{\alpha} \cap \mathbf{b}_{u_{\alpha}}^{1}=\mathbf{x}_{\alpha} \cap \bigcap_{\gamma \in u_{\alpha}} \mathbf{b}_{\gamma}^{1}$ to $\mathbf{x}_{\alpha} \cap \mathbf{b}_{w}$. Equation (ff) would imply

$$
\hat{h}_{w}\left(\mathbf{a}_{* *} \cap \bigcap_{\alpha \in v}\left(\mathbf{x}_{\alpha} \cap \mathbf{b}_{u_{\alpha}}^{1}\right)\right)=\mathbf{a}_{* *} \cap \bigcap_{\alpha \in v} \mathbf{x}_{\alpha} \cap \mathbf{b}_{w}=\mathbf{a}_{* *}>0 .
$$

Equation (还) follows.]

It remains to prove (If). In fact $\mathbf{a}_{* *}=\mathbf{a}_{*}$ already works. Remember that we are in a very special case: $v=u \subseteq \mathcal{U}$, so $\left\langle u_{\alpha}: \alpha \in v\right\rangle$ is constantly equal to $u_{\oplus}$, so $w=\bigcup_{\alpha \in v} u_{\alpha}=u_{\oplus}$ (!). So $\mathbf{b}_{w}=\mathbf{b}_{u_{\oplus}}$. Meanwhile, from 10.19(1) we have $\mathbf{x}_{\alpha} \leq \mathbf{b}_{u_{\alpha}}$ for any $\alpha<\kappa$, so $\mathbf{a}_{*} \leq \mathbf{x}_{\alpha} \leq \mathbf{b}_{u_{\oplus}}$ for $\alpha \in u$.

This completes the proof of the $(\kappa, \mathcal{I}, \bar{m})$-pattern transfer, and so the proof of the Claim.

Remark 10.21. Although 10.20 assumes $\theta<\kappa$, the same proof will show another hypothesis also works: $\kappa \leq \theta \leq \lambda$ but for some $\mu<\kappa$ we have $\left(\forall u \in[\theta]^{<\aleph_{0}}\right)\left[\mathfrak{B}_{\mathfrak{a}}=\right.$ $\left.\mathbf{b}_{u}=\bigcap\left\{\mathbf{b}_{\{\alpha\}}: \alpha \in(u \backslash \mu)\right\} \cap \mathbf{b}_{u \cap \mu}\right]$.

Proof. Suppose we are given such a $\mu$. In equation (国) replace $\theta$ by $\mu$ and then let $\mathbf{a}_{\alpha}^{1}=\mathbf{x}_{\alpha} \cap \mathbf{b}_{u_{\oplus}} \cap \bigcap_{\alpha \in u_{\alpha} \backslash \mu} \mathbf{b}_{\alpha}$ for $\alpha \in \mathcal{U}_{1}$, and verify that $\left\langle\mathbf{a}_{\alpha}^{1}: \alpha \in \mathcal{U}\right\rangle$ is as required, for $j=1$ (or 0 ).

Theorem 10.22 (Realizing $T_{\mathfrak{m}}$-types). Assume $\mathfrak{a} \in \mathrm{AP}$ and $\mathfrak{b}$ is a $(\theta, T, \overline{\mathbf{b}})$ extension of $\mathfrak{a}$ where $\theta \leq \lambda, T=T_{\mathfrak{m}}$ for some $\mathfrak{m} \in \mathcal{M}$, and $\overline{\mathbf{b}}$ is a possibility pattern arising from a type of the form

$$
\left\{Q_{\rho_{*}}(x)\right\} \cup\left\{R\left(x, a_{\beta}\right): \beta<\theta\right\}
$$

for some $\rho_{*} \in \mathcal{T}_{1}$. Then $\left(\mathfrak{B}_{\mathfrak{a}}, \mathfrak{B}_{\mathfrak{b}}\right)$ satisfies the $(\kappa, \mathcal{I}, \overline{\mathfrak{m}})$-pattern transfer property.

Proof. By hypothesis our $T_{\mathfrak{m}}$ is given by some

$$
\mathfrak{m}=\mathfrak{m}(\bar{m}, \bar{E}, \xi) \in \mathcal{M}
$$

This proof is similar to 10.20, so we will be lighter on motivation already given there. The main difference is that now we will have to handle the larger $\theta$ by leveraging an understanding of the type in our theory $T_{\mathfrak{m}}$.

Recall notation from 10.12, $\overline{\mathbf{b}}=\left\langle\mathbf{b}_{u}: u \in[\theta]^{\left\langle\aleph_{0}\right.}\right\rangle$, a sequence of elements of $\mathfrak{B}_{\mathfrak{a}}$, is the problem which was solved in $\mathfrak{b}$, and was given along with the data of the form $\mathbf{a}[\psi] \in \mathfrak{B}_{\mathfrak{a}}$; and $\mathbf{b}_{\alpha}^{1}, \mathbf{b}_{u}^{1}$ in $\mathfrak{B}_{\mathfrak{b}}$ are from the formal solution.

\footnotetext{
${ }^{21}$ Presently, it would be both sufficient and possible to show just that $\bigcap_{\alpha \in v} \mathbf{x}_{\alpha} \leq \mathbf{b}_{w}$, but this a priori more general criterion will be useful later.
} 
We aim to prove the pattern transfer property, 8.12. Suppose we are given $\left\langle\mathbf{a}_{\alpha}^{2}: \alpha<\kappa\right\rangle$ with each $\mathbf{a}_{\alpha}^{2} \in \mathfrak{B}_{\mathfrak{b}}^{+}$. First, following 10.19(1), for each $\alpha<\kappa$ we choose $\mathbf{i}_{\alpha}=\left(\mathbf{x}_{\alpha}, u_{\alpha}, n_{\alpha},\left\langle u_{\alpha, \ell}: \ell<n_{\alpha}\right\rangle\right)$ as there, so $\mathbf{x}_{\alpha} \leq \mathbf{b}_{u_{\alpha}}$ for each $\alpha<\kappa$, and

$$
\mathfrak{B}_{\mathfrak{b}} \models 0<\mathbf{x}_{\alpha} \cap \mathbf{b}_{u_{\alpha}}^{1} \cap \bigcap_{\ell<m_{\alpha}}\left(-\mathbf{b}_{u_{\alpha, \ell}}^{1}\right) \leq \mathbf{a}_{\alpha}^{2} .
$$

By way of orientation: recall that each $u_{\alpha}$ is a finite subset of $\theta$, and $\mathbf{b}_{u_{\alpha}}$ can be thought of as the $\mathbf{j}$-image of $B_{u_{\alpha}}=\left\{t \in I: M[t] \models \exists x \bigwedge_{\beta \in u_{\alpha}} Q_{\rho_{*}}(x) \wedge R\left(x, a_{\beta}[t]\right)\right\}$ in some enveloping ultrapower, as in $\$ 7$. In this language, we can say

$$
\mathbf{b}_{u_{\alpha}} \leq \mathbf{a}\left[(\exists x) \bigwedge\left\{Q_{\rho_{*}}(x) \wedge R\left(x, a_{\gamma}\right): \gamma \in u_{\alpha}\right\}\right]
$$

noting that on different positive $\mathbf{a} \cap \mathbf{b}_{u_{\alpha}}$, different additional constraints may be in force on the the $a_{\beta}$ 's, for instance as described by the $\mathbf{a}[\psi]$ 's.

Specifically, in our present context, we make the following upgrade to 10.19(1). For $\alpha<\kappa$, for each of the finitely many atomic formulas $\psi \in\left\{a_{\gamma}=a_{\beta}, \mathcal{Q}(x)\right.$, $\left.\mathcal{P}\left(a_{\beta}\right): \gamma, \beta \in u_{\alpha}\right\}$, we may without loss of generality assume $\mathbf{x}_{\alpha}$ from (b) decides it, that is, without loss of generality

$$
\mathbf{x}_{\alpha} \leq \mathbf{a}[\psi] \text { or } \mathbf{x}_{\alpha} \cap \mathbf{a}[\psi]=\emptyset \text { for each such } \psi .
$$

We can now be sure by (C) that

$$
\mathbf{x}_{\alpha} \leq \mathbf{a}\left[Q_{\rho_{*}}(x)\right] \text {, and also } \mathbf{x}_{\alpha} \leq \mathbf{a}\left[\mathcal{P}\left(a_{\gamma}\right)\right] \text { for each } \gamma \in u_{\alpha} .
$$

By $10.19(2)$, there are $\mathcal{U} \in[\kappa]^{\kappa}, w_{*}, u_{*}, n_{*},\left\langle u_{\ell}^{*}: \ell<n_{*}\right\rangle$, and let us add, $m_{*}$ such that for every $\alpha \in \mathcal{U}: n_{\alpha}=n_{*}, u_{\alpha} \cap w_{*}=u_{*}, u_{\alpha, \ell} \cap w_{*}=u_{\ell}^{*}$, and $\left|u_{\alpha}\right|=m_{*}$. Let

$$
\mathbf{a}_{\alpha}^{1}=\mathbf{x}_{\alpha} \text { for each } \alpha \in \mathcal{U} \text {. }
$$

Then for each $\alpha$ we have $\mathbf{a}_{\alpha}^{1} \leq_{\text {proj }} \mathbf{a}_{\alpha}^{2}$, by 10.19 (3).

As $\mathfrak{a} \in \mathrm{AP}$, the $(\kappa, \mathcal{I}, \bar{m})$-c.c. holds for $\mathfrak{B}_{\mathfrak{a}}$, so, given the sequence of elements $\overline{\mathbf{a}}^{1}=\left\langle\mathbf{a}_{\alpha}^{1}: \alpha \in \mathcal{U}\right\rangle=\left\langle\mathbf{x}_{\alpha}: \alpha \in \mathcal{U}\right\rangle$, fix

$$
j_{1}<\omega, \quad \mathcal{U}_{1} \in[\mathcal{U}]^{\kappa}, \quad A_{1} \in \mathcal{I}
$$

such that $\oplus$ of 8.2 holds. (We will not really use this additional strength in the present proof, but it is natural to add.) We aim to show that the pattern transfers for

$$
j=j_{1}+2, \quad \mathcal{U}_{1}, \quad A=A_{1} \cup\left\{\ell: \ell \leq j+m_{*}+n_{*}\right\} \cup \xi^{-1}\{1\}, \quad \overline{\mathbf{a}}^{1}\left\lceil\mathcal{U}_{1}\right.
$$

recalling $\xi$ is the level function for $T_{\mathfrak{m}}$ from (国), so adding $\xi^{-1}\{1\}$ to $A$ amounts to ensuring $n$ is not an active level for $T_{\mathfrak{m}}$. Fix, then, $n, u, i, \mathbf{a}_{*}$ such that $n \in \omega \backslash A$, $u$ is a finite subset of $\mathcal{U}_{1}$, and $\mathbf{a}_{*} \in \mathfrak{B}_{\mathfrak{a}}^{+}$, and together they satisfy

$$
m_{n} /\left(m_{n}^{\circ}\right)^{n^{i}}<|u|<m_{n} \text { and } \mathfrak{B}_{\mathfrak{a}} \models 0<\mathbf{a}_{*} \leq \bigcap_{\alpha \in u} \mathbf{x}_{\alpha} .
$$

Note that by definition of $A$, necessarily $n>\max \left\{m_{*}, n_{*}\right\}$. We would like to find $v \subseteq u$ of an "appropriate size" [i.e. satisfying [8.12(4)(f)(ii)] so that $\mathfrak{B}_{\mathfrak{b}} \models$ $\bigcap_{\alpha \in v} \mathbf{a}_{\alpha}^{2} \cap \mathbf{a}_{*}>0$. First observe that by 10.19(4), it would suffice to show that for some $v \subseteq u$ of an appropriate size,

$$
\mathfrak{B}_{\mathfrak{b}} \models \bigcap_{\alpha \in v}\left(\mathbf{x}_{\alpha} \cap \mathbf{b}_{u_{\alpha}}^{1}\right) \cap \mathbf{a}_{*}>0
$$


and for (h), just as in 10.20(f), it would suffice to show that for $w(v)=\bigcup_{\alpha \in v} u_{\alpha}$ and some $\mathbf{a}_{* *} \in \mathfrak{B}_{\mathfrak{a}}$ with $0<\mathbf{a}_{* *} \leq \mathbf{a}_{*}$, we have

$$
\mathbf{a}_{* *} \leq \mathbf{b}_{w(v)} .
$$

In search of a suitable $v$, we make the following additional reductions. First, since $\mathcal{T}_{2, n}$ is finite, we may replace $\mathbf{a}_{*}$ by a smaller positive element $\mathbf{a}_{* *} \in \mathfrak{B}_{\mathfrak{a}}$ if necessary [i.e. by decreasing $\mathbf{a}_{*}$ to $\mathbf{a}_{* *}$ to decide a finite partition of $\mathbf{a}\left[\mathcal{P}\left(a_{\beta}\right)\right]$ for each of the finitely many $\alpha \in u$ and $\beta \in u_{\alpha}$, remembering by equation (ef) that $\mathbf{a}_{*} \leq \mathbf{x}_{\alpha} \leq$ $\mathbf{a}\left[\mathcal{P}\left(a_{\beta}\right)\right]$ for $\left.\alpha \in u, \beta \in u_{\alpha}\right]$ so that for each $\alpha \in u$ and each $\gamma \in u_{\alpha}$, there is one and only one $\eta_{\gamma} \in \mathcal{T}_{2, n}$ such that

$$
\mathbf{a}_{* *} \leq \mathbf{a}\left[P_{\eta_{\gamma}}\left(a_{\gamma}\right)\right] .
$$

Informally, for each relevant formula $R\left(x, a_{\gamma}\right), \mathbf{a}_{* *}$ decides which branch $a_{\gamma}$ belongs to at level $n$. Next, we choose a subset of $u$ on which this sequence of choices is constant. That is, for each $\alpha \in u$, let $\left\langle\gamma(\alpha, \ell): \ell<m_{*}\right\rangle$ list $u_{\alpha}$ in strictly increasing order, necessarily without repetitions. Let $\left\langle\eta_{\gamma(\alpha, \ell)}: \ell\left\langle m_{*}\right\rangle\right.$ be the corresponding sequence of elements of $\mathcal{T}_{2, n}$ chosen by $\mathbf{a}_{* *}$. Recalling $6.15(c),\left|\mathcal{T}_{2, n}\right|=m_{n}^{\circ}$, so there are at most $\left(m_{n}^{\circ}\right)^{m_{*}}$ possible such sequences, and so there are $\left\langle\nu_{\ell}: \ell\left\langle m_{*}\right\rangle\right.$ and $v \subseteq u$ such that for every $\alpha \in v,\left\langle\eta_{\gamma(\alpha, \ell)}: \ell<m_{*}\right\rangle=\left\langle\nu_{\ell}: \ell<m_{*}\right\rangle$ and

$$
|v| \geq|u| /\left(m_{n}^{\circ}\right)^{m_{*}} \geq|u| /\left(m_{n}^{\circ}\right)^{n} \geq m_{n} /\left(m_{n}^{\circ}\right)^{n^{i} \cdot n}=m_{n} /\left(m_{n}^{\circ}\right)^{n^{i+1}} .
$$

Such a $v$ therefore has an appropriate size. There is no harm (and there will be a later help) in replacing $v$ by a subset if necessary so that we know its size exactly:

$$
|v|=m_{n} /\left(m_{n}^{\circ}\right)^{n^{i+1}} .
$$

Fix such a $v$ for the rest of the proof, and let $w=w(v)$. Note that, liberally,

$$
|w|=m_{*} \cdot\left(m_{n} /\left(m_{n}^{\circ}\right)^{n^{i+1}}\right) \leq m_{n} .
$$

It remains to show equation (ii) holds for our $\mathbf{a}_{* *}$ and this $w$.

When we ask whether $\mathbf{a}_{* *} \leq \mathbf{b}_{w}$, we are asking whether the decisions already made by $\mathbf{a}_{* *}$ are enough to guarantee consistency of

$$
\left\{Q_{\rho_{*}}(x) \wedge R\left(x, a_{\gamma}\right) \wedge P_{\eta_{\gamma}}\left(a_{\gamma}\right): \alpha \in u, \gamma \in u_{\alpha}\right\}
$$

e.g. for any possible further choice of leaves that the $a_{\gamma}$ 's may belong to. For each individual $\alpha \in u$, we know $\mathbf{a}_{* *} \leq \mathbf{b}_{u_{\alpha}}$, so the answer is yes for the smaller set

$$
\left\{Q_{\rho_{*}}(x) \wedge R\left(x, a_{\gamma}\right) \wedge P_{\eta_{\gamma}}\left(a_{\gamma}\right): \gamma \in u_{\alpha}\right\} \text {. }
$$

Recalling the constant sequence $\left\langle\nu_{\ell}: \ell\left\langle m_{*}\right\rangle\right.$ from the definition of $v$, we may rewrite equation (ㅁ) to say also that the answer is yes for

$$
\left\{Q_{\rho_{*}}(x) \wedge R\left(x, a_{\gamma(\alpha, \ell)}\right) \wedge P_{\nu_{\ell}}\left(a_{\gamma(\alpha, \ell)}\right): \ell<m\right\} .
$$

Thus, for some $\rho \in \mathcal{T}_{1, n}$ such that $\rho_{*} \unlhd \rho$ [recalling $n>\operatorname{lgn}\left(\rho_{*}\right)$ by the definition of $A]$ we have that

$$
\{\rho\} \times\left\{\nu_{\ell}: \ell<m_{*}\right\} \subseteq \mathcal{R}_{n} .
$$

[Note that $\alpha$ doesn't really matter in the choice of $\rho$ : the axioms of $T_{\mathfrak{m}}$ imply that for $\rho$ from $\mathcal{T}_{1, n}$ and $\left\langle\nu_{\ell}: \ell<m_{*}\right\rangle$ a sequence of elements from $\mathcal{T}_{2, n}$, the set $\left\{Q_{\rho}(x) \wedge R\left(x, y_{\ell}\right) \wedge P_{\nu_{\ell}}\left(y_{\ell}\right): \ell<m_{*}\right\}$ is consistent if and only if $\{\rho\} \times\left\{\nu_{\ell}: \ell<m_{*}\right\}$ is a complete bipartite graph in $\mathcal{R}_{n}$. Fix any $\rho$ which has this property; there may be many to choose from.] 
For each $\gamma \in w$, choose some $\eta_{\gamma}^{*} \in \lim \left(\mathcal{T}_{2}\right)$ such that if $\gamma=\gamma(\alpha, \ell)$ then $\nu_{\ell} \unlhd \eta_{\gamma}^{*}$ [i.e., this leaf extends the choice made for $a_{\gamma}$ by $\mathbf{a}_{* *}$ ]. We shall prove

(r) there is $\varrho \in \lim \left(\mathcal{T}_{1}\right)$ such that $\rho \unlhd \varrho$ and $\left(\varrho, \eta_{\gamma}^{*}\right) \in \mathcal{R}_{\infty}$ for every $\gamma \in w$.

This will suffice for equation (iil) and so will finish the proof.

To prove (피) we shall choose an increasing sequence $\varrho_{n+t} \in \mathcal{T}_{1, n+t}$ by induction on $t \geq n$. The case of $t=0$ is immediate: let $\varrho_{n}=\rho$, and then $\left(\varrho_{n}, \eta_{\gamma}^{*} \uparrow_{n}\right) \in \mathcal{R}_{n}$ for each $\gamma \in w$ by choice of $\rho$ (and definition of $v$ ). For $t=1$, recall the choice of $n \in \omega \backslash A$ means $\xi(n)=0$, so there are no new constraints (it is a "lazy level"). Let $\varrho_{n+1}$ be any element of $\mathcal{T}_{1, n+1}$ which extends $\varrho_{n}$, and it will remain true that $\left(\varrho_{n+1},\left.\eta_{\gamma}^{*}\right|_{n+1}\right) \in \mathcal{R}_{n+1}$ for each $\gamma \in w$. [Note that the lazy level plays an essential role here: $|w| \leq m_{n}$, and elements of $\mathcal{T}_{2, n}$ have $m_{n}$ immediate successors, recalling 6.15. improvements to the upper-bound calculation (m) won't bring the size down to something small at this level.] For $t>1$, the coast is now clear: whether $\xi(t)=0$ or 1 , recalling $(\mathrm{m})$, the set $w$ is now very comfortably small in the sense of 6.2

$$
|w| \leq m_{n}<m_{n+1}^{\circ} \leq\left(m_{n+t-1}^{\circ}\right)^{(n+t-1)^{(n+t-1)}} .
$$

So we may apply Claim 6.17(1), which ensures (using $n+t-1$ here for $k$ there) that given $\left\{\left.\eta_{\gamma}^{*}\right|_{n+t-1}: \gamma \in w\right\} \subseteq \mathcal{T}_{2, n+t-1}$ along with equation (鴫) and our inductive hypothesis, there is $\varrho_{n+t} \in \mathcal{T}_{1, n+t}$ extending $\varrho_{n+t-1}$ such that $\left(\varrho_{n+t},\left.\eta_{\gamma}^{*}\right|_{n+t}\right) \in$ $\mathcal{R}_{n+t}$ for each $\gamma \in w$. So we can carry the induction. Let $\varrho \in \lim \left(\mathcal{T}_{1}\right)$ be the unique element such that $\varrho \uparrow i=\varrho_{i}$ for all $i<\omega$. This proves (II), so proves (il), and so finishes the proof of the theorem.

For our third and final case of realizing types, in the case of the random graph, the theory is simple enough that we will construct the refinement directly. The proof is like [23] Theorem 3.2, though there are still many differences. The proof is mainly a matter of keeping track of equality.

Definition 10.23. For our purposes, " $\overline{\mathbf{b}}=\left\langle\mathbf{b}_{u}: u \in[\theta]^{<\aleph_{0}}\right\rangle$ is a problem for the theory of the random graph" in $\mathfrak{B}$ means that $\overline{\mathbf{b}}$ arises from some partial type $\left\{R\left(x, a_{\gamma}\right)^{\mathbf{t}(\gamma)}: \gamma<\theta, \mathbf{t}: \theta \rightarrow\{0,1\}\right\}$ in some enveloping ultrapower 22 and so reflecting this, $\overline{\mathbf{b}}$ is given along with $\left\{\mathbf{a}\left[a_{\beta} \neq a_{\gamma}\right]: \beta, \gamma<\theta\right\}$ and the sequence of truth values $\{\mathbf{t}(\beta): \beta<\theta\}$, and for each $u \in[\theta]<\aleph_{0}$,

$$
\mathbf{b}_{u}=\bigcap\left\{\mathbf{a}\left[a_{\beta} \neq a_{\gamma}\right]: \beta, \gamma \in u, \mathbf{t}(\beta) \neq \mathbf{t}(\gamma)\right\}
$$

translating the fact that in the enveloping ultrapower, at a given index $i \in I$, a subset $\left\{R\left(x, a_{\gamma}[i]\right)^{\mathbf{t}(\gamma)}: \gamma \in u\right\}$ of the background type is consistent if for all $\gamma, \beta \in u$, $\mathbf{t}(\gamma) \neq \mathbf{t}(\beta) \Longrightarrow \mathbf{a}_{\gamma}[i] \neq \mathbf{a}_{\beta}[i]$. In keeping with our earlier notation, we will drop parentheses on singletons, and so write $\mathbf{b}_{\gamma}$ for $\mathbf{b}_{\{\gamma\}}$. Note that each $\mathbf{b}_{\gamma}=1_{\mathfrak{B}}$.

Lemma 10.24 (Realizing random graph types). Suppose $\mathfrak{a} \in \mathrm{AP}$ and let $\theta \leq \lambda$. Suppose $\overline{\mathbf{b}}=\left\langle\mathbf{b}_{u}: u \in[\theta]^{<\aleph_{0}}\right\rangle$ is a problem for the theory of the random graph, so a sequence of elements of $\mathfrak{B}_{\mathfrak{a}}^{+}$, indeed of $\mathcal{D}_{\mathfrak{a}}$. Then there is $\mathfrak{b} \in \mathrm{AP}$ such that

(1) $\mathfrak{a} \leq_{\text {AP }} \mathfrak{b}$, and

(2) there is a solution of $\overline{\mathbf{b}}$ in $\mathfrak{b}$, i.e. there are $\mathbf{b}_{\alpha}^{1} \in \mathcal{D}_{\mathfrak{b}}$ for $\alpha<\theta$ such that $\mathfrak{B}_{\mathfrak{b}} \models \bigcap\left\{\mathbf{b}_{\alpha}^{1}: \alpha \in u\right\} \leq \mathbf{b}_{u}$ for $u \in[\theta]^{<\aleph_{0}}$.

\footnotetext{
${ }^{22}$ Here of course $R(x, y)$ is the edge relation for the random graph, unlike elsewhere.
} 
Proof. Suppose we are given $\overline{\mathbf{b}}$ along with the supporting data mentioned in 10.23 We start by defining some auxiliary objects, which aim to keep track of equalities. For each $\gamma<\theta$, call $\mathbf{x} \in \mathfrak{B}_{\mathfrak{a}}^{+}$collapsed for $\gamma$ if for some $\beta \leq \gamma$,

$$
0<\mathbf{x} \leq \mathbf{a}\left[a_{\gamma}=a_{\beta}\right] \text { but for all } \delta<\beta, \mathbf{x} \cap \mathbf{a}\left[a_{\gamma}=a_{\delta}\right]=0_{\mathfrak{B}_{\mathfrak{a}}} .
$$

Note that for every $\gamma<\theta$ and $\mathbf{a} \in \mathfrak{B}_{\mathfrak{a}}^{+}$there exists $\mathbf{x}$ with $0<\mathbf{x} \leq \mathbf{a}$ which is collapsed for $\gamma$, because the ordinals are well ordered. So we may try to choose for each $\gamma<\theta$ a maximal antichain [supporting $\mathbf{b}_{\gamma}$, though this is trivial as each $\mathbf{b}_{\gamma}=1_{\mathfrak{B}_{\mathfrak{a}}}$ ] consisting of disjoint nonzero elements $\mathbf{a}_{\gamma, \epsilon}$ each of which is collapsed for $\gamma$, by induction on $\epsilon<\kappa$. As $\mathfrak{B}_{\mathfrak{a}}$ satisfies the $\kappa$-c.c. we stop at some ordinal below $\kappa$. Renumbering, we may assume this maximal antichain is indexed by a cardinal $\mu_{\gamma}<\kappa$. Write the result as:

$$
\left\langle\left(\mathbf{a}_{\gamma, \epsilon}, \beta_{\gamma, \epsilon}\right): \epsilon<\mu_{\gamma}\right\rangle
$$

where $\beta_{\gamma, \epsilon}$ is the minimal $\beta \leq \gamma$ such that $\mathbf{a}_{\gamma, \epsilon} \leq \mathbf{a}\left[a_{\gamma}=a_{\beta}\right]$, in the sense of (b). Without loss of generality, we may assume that $\epsilon<\epsilon^{\prime}$ implies $\beta_{\gamma, \epsilon} \neq \beta_{\gamma, \epsilon^{\prime}}$ : if not, combine all elements of the antichain with the same $\beta$ and renumber.

On the relation of these antichains to each other: fixing $\gamma<\theta$, suppose $\beta=$ $\beta_{\gamma, \epsilon}$. Observe that there is one and only one $\delta<\mu_{\beta}$ such that $\mathbf{a}_{\gamma, \epsilon} \cap \mathbf{a}_{\beta, \delta}>0$. [There is at least one such $\delta$ because $\left\langle\mathbf{a}_{\beta, \delta}: \delta<\mu_{\beta}\right\rangle$ is a maximal antichain. So $0<\mathbf{a}_{\gamma, \epsilon} \cap \mathbf{a}_{\beta, \delta} \leq \mathbf{a}\left[a_{\gamma}=a_{\beta_{\gamma, \epsilon}}\right] \cap \mathbf{a}\left[a_{\beta}=a_{\beta_{\beta, \delta}}\right]$ but since $\beta_{\gamma, \epsilon}$ is minimal and $\beta_{\beta, \delta} \leq \beta \leq \beta_{\gamma, \epsilon}$, necessarily they are all equal. There is only one such $\delta$ by the last sentence of the previous paragraph.] So necessarily $\mathbf{a}_{\gamma, \epsilon} \leq \mathbf{a}_{\beta, \delta}$. For $\gamma<\theta$, $\epsilon<\mu_{\gamma}$ let us write $\zeta_{\gamma, \epsilon}<\mu_{\beta_{\gamma, \epsilon}}$ for this unique value so that $\mathbf{a}_{\gamma, \epsilon} \leq \mathbf{a}_{\beta_{\gamma, \epsilon}, \zeta_{\gamma, \epsilon}}$. Note moreover that

(d) if $\beta_{\gamma_{1}, \epsilon_{1}}=\beta_{\gamma_{2}, \epsilon_{2}}=\beta$ (for $\gamma_{1}$ not necessarily equal to $\gamma_{2}$ ) then $\zeta_{\gamma_{1}, \epsilon_{1}}=\zeta_{\gamma_{2}, \epsilon_{2}}$

by a similar argument, since there is at most one $\zeta<\mu_{\beta}$ such that $\beta_{\beta, \zeta}=\beta$.

To summarize, we may write the elements of (ㄷ) as triples:

$$
\left\langle\left(\mathbf{a}_{\gamma, \epsilon}, \beta_{\gamma, \epsilon}, \zeta_{\gamma, \epsilon}\right): \epsilon<\mu_{\gamma}\right\rangle \text {. }
$$

where we can informally express this information as saying that on $\mathbf{a}_{\gamma, \epsilon}, a_{\gamma}$ collapses to $a_{\beta}$ where $\beta=\beta_{\gamma, \epsilon}$, and $\zeta=\zeta_{\gamma, \epsilon}$ tells us $\mathbf{a}_{\gamma, \epsilon}$ is below the $\zeta$-th element of the antichain for $\beta$.

We now define the Boolean algebra $\mathfrak{B}_{\mathfrak{b}}$. Let $\left\{\mathbf{c}_{\gamma, \epsilon}: \gamma<\theta, \epsilon<\mu_{\gamma}\right\}$ be new elements. Let $\mathfrak{B}_{\mathfrak{b}}$ be the completion of the Boolean algebra $\mathfrak{B}_{\mathfrak{b}}^{0}$ generated by

$$
\mathcal{X}=\mathfrak{B}_{\mathfrak{a}} \cup\left\{\mathbf{c}_{\gamma, \epsilon}: \gamma<\theta, \epsilon<\mu_{\gamma}\right\}
$$

freely except:

(i) the equations in $\mathfrak{B}_{\mathfrak{a}}$

(ii) $\mathbf{c}_{\gamma, \epsilon_{1}} \cap \mathbf{c}_{\gamma, \epsilon_{2}}=0$ when $\epsilon_{1}<\epsilon_{2}<\mu_{\gamma}$

(iii) $\mathbf{c}_{\gamma_{1}, \epsilon_{1}} \cap \mathbf{c}_{\gamma_{2}, \epsilon_{2}}=0$ when $\epsilon_{1}<\mu_{\gamma_{1}}, \epsilon_{2}<\mu_{\gamma_{2}}, \mathbf{t}\left(\gamma_{1}\right) \neq \mathbf{t}\left(\gamma_{2}\right)$ and $\beta_{\gamma_{1}, \epsilon_{1}}=\beta_{\gamma_{2}, \epsilon_{2}}$.

Let us verify that without loss of generality $\mathfrak{B}_{\mathfrak{a}} \subseteq \mathfrak{B}_{\mathfrak{b}}$. Let $h$ be the identity on $\mathfrak{B}_{\mathfrak{a}}$ and map $\mathbf{c}_{\gamma, \epsilon}$ (for $\gamma<\theta, \epsilon<\mu_{\gamma}$ ) to $0_{\mathfrak{B}_{\mathfrak{a}}}$. So $h$ is a function from $\mathcal{X}$ onto $\mathfrak{B}_{\mathfrak{a}}$ respecting the equations, hence has an extension $\hat{h}$ which is a homomorphism from $\mathfrak{B}_{\mathfrak{b}}$ into $\mathfrak{B}_{\mathfrak{a}}^{0}$ such that $\hat{h}\left\lceil\mathfrak{B}_{\mathfrak{a}}=\mathrm{id}_{\mathfrak{B}_{\mathfrak{a}}}\right.$, so we are done.

Let us verify that $\mathfrak{B}_{\mathfrak{a}} \lessdot \mathfrak{B}_{\mathfrak{b}}$. Let $\mathbf{c} \in \mathfrak{B}_{\mathfrak{b}}^{+}$and we shall find a projection from $\mathfrak{B}_{\mathfrak{b}}$ onto $\mathfrak{B}_{\mathfrak{a}}$ mapping $\mathbf{c}$ to a positive element. We can replace $\mathbf{c}$ by any $\mathbf{c}^{\prime} \leq \mathbf{c}$ 
which is not zero. As the generators are dense in the completion 23 without loss of generality for some $\mathbf{a} \in \mathfrak{B}_{\mathfrak{a}}^{+}$, finite $u, v \subseteq \theta$, a function $f$ with finite domain $u \subseteq \theta$ such that $f(\gamma)<\mu_{\gamma}$ for $\gamma \in u$, and finite $w_{\beta} \subseteq \mu_{\beta}$ for $\beta \in v$, we have

$$
0<\bigcap\left\{\mathbf{c}_{\gamma, f(\gamma)}: \gamma \in u\right\} \cap \bigcap\left\{-\mathbf{c}_{\beta, \epsilon}: \beta \in v, \epsilon \in w_{\beta}\right\} \cap \mathbf{a} \leq \mathbf{c}
$$

where necessarily $\{(\gamma, f(\gamma)): \gamma \in u\} \cap\left\{(\beta, \epsilon): \beta \in v, \epsilon \in w_{\beta}\right\}=\emptyset$, and if $\gamma_{1} \neq$ $\gamma_{2} \in u$ and $\mathbf{t}\left(\gamma_{1}\right) \neq \mathbf{t}\left(\gamma_{2}\right)$ then $\beta_{\gamma_{1}, f\left(\gamma_{1}\right)} \neq \beta_{\gamma_{2}, f\left(\gamma_{2}\right)}$. If not, $\mathbf{c}_{\gamma_{1}, f\left(\gamma_{1}\right)} \cap \mathbf{c}_{\gamma_{2}, f\left(\gamma_{2}\right)}=0$, which contradicts the intersection being positive.] Define $h: \mathcal{X} \rightarrow \mathfrak{B}_{\mathfrak{a}}$ so that $h$ is the identity on $\mathfrak{B}_{\mathfrak{a}}, h\left(\mathbf{c}_{\gamma, f(\gamma)}\right)=\mathbf{a}$ for $\gamma \in u$, and $h\left(\mathbf{c}_{\gamma, \epsilon}\right)=0_{\mathfrak{B}_{\mathfrak{a}}}$ for $(\gamma, \epsilon) \notin$ $\{(\gamma, f(\gamma)): \gamma \in u\}$. By the note after equation (ffl), $h$ respects the equations in (iii). So $h$ extends to a homomorphism $\hat{h}$ from $\mathfrak{B}_{\mathfrak{b}}$ onto $\mathfrak{B}_{\mathfrak{a}}$ which indeed is the identity on $\mathfrak{B}_{\mathfrak{a}}$ and sends $\mathbf{c}$ to a positive element.

Now we define our multiplicative refinement. First, for $\gamma<\theta, \epsilon<\mu_{\gamma}$ let $\mathbf{b}_{\gamma, \epsilon}^{1}=$ $\mathbf{a}_{\gamma, \epsilon} \cap \mathbf{c}_{\gamma, \epsilon}$. Then for every $\gamma<\theta$ let

$$
\mathbf{b}_{\gamma}^{1}=\bigcup\left\{\mathbf{b}_{\gamma, \epsilon}^{1}: \epsilon<\mu_{\gamma}\right\} .
$$

We have to check first that

$$
u \in[\theta]^{<\aleph_{0}} \Longrightarrow \bigcap_{\gamma \in u} \mathbf{b}_{\gamma}^{1} \leq \mathbf{b}_{u}
$$

and second that there is an ultrafilter $\mathcal{D}$ on $\mathfrak{B}_{\mathfrak{b}}$ extending $\mathcal{D}_{\mathfrak{a}}$ such that

$$
\mathbf{b}_{\gamma}^{1} \in \mathcal{D} \quad \text { for each } \gamma<\theta .
$$

First we check equation (h). It will suffice to consider $u=\left\{\gamma_{1}, \gamma_{2}\right\}, \gamma_{1} \neq \gamma_{2}$. [If $u=$ $\{\gamma\}$ remember that $\mathbf{b}_{\gamma}=1_{\mathfrak{B}}$. If $|u|>2$ remember that $\mathbf{b}_{u}=\bigcap_{v \subseteq u,|v|=2} \mathbf{b}_{v}$, since consistency of the corresponding formulas depends only on instances of equality.] So it suffices to prove that for $\left(\epsilon_{1}, \epsilon_{2}\right) \in \mu_{\gamma_{1}} \times \mu_{\gamma_{2}}$,

$$
\mathbf{b}_{\gamma_{1}, \epsilon_{1}}^{1} \cap \mathbf{b}_{\gamma_{2}, \epsilon_{2}}^{1} \leq \mathbf{b}_{\left\{\gamma_{1}, \gamma_{2}\right\}} \text {. }
$$

There are three cases 24 Case 1. $\mathbf{t}\left(\gamma_{1}\right)=\mathbf{t}\left(\gamma_{2}\right)$. Then $\mathbf{b}_{\left\{\gamma_{1}, \gamma_{2}\right\}}=1_{\mathfrak{B}}$, so the inequality holds trivially. Case 2. $\mathbf{t}\left(\gamma_{1}\right) \neq \mathbf{t}\left(\gamma_{2}\right), \beta_{\gamma_{1}, \epsilon_{1}} \neq \beta_{\gamma_{2}, \epsilon_{2}}$. Then $\mathbf{a}_{\gamma_{1}, \epsilon_{1}} \cap$ $\mathbf{a}_{\gamma_{2}, \epsilon_{2}} \leq \mathbf{a}\left[a_{\gamma_{1}} \neq a_{\gamma_{2}}\right]$ which suffices. Case 3. $\mathbf{t}\left(\gamma_{1}\right) \neq \mathbf{t}\left(\gamma_{2}\right), \beta_{\gamma_{1}, \epsilon_{1}}=\beta_{\gamma_{2}, \epsilon_{2}}$ (call it $\beta)$. Then

$$
\mathbf{b}_{\gamma_{1}, \epsilon_{1}}^{1} \cap \mathbf{b}_{\gamma_{2}, \epsilon_{2}}^{1} \leq \mathbf{c}_{\gamma_{1}, \epsilon_{1}} \cap \mathbf{c}_{\gamma_{2}, \epsilon_{2}}=0_{\mathfrak{B}}
$$

by clause (iii) in the definition of $\mathfrak{B}_{\mathfrak{b}}$. This completes the verification of (h) .

Next we verify equation (ii). For this we should show there is an ultrafilter $\mathcal{D}$ on $\mathfrak{B}_{\mathfrak{b}}$ extending $\mathcal{D}_{\mathfrak{a}} \cup\left\{\mathbf{b}_{\gamma}^{1}: \gamma<\theta\right\}$. As in 10.17 it suffices to prove that given a finite $u \subseteq \theta$ and $\mathbf{d} \in \mathcal{D}_{\mathfrak{a}}$,

$$
\mathbf{b}_{u}^{1}=\bigcap\left\{\mathbf{b}_{\gamma}^{1}: \gamma \in u\right\} \text { is not disjoint to } \mathbf{d} .
$$

\footnotetext{
${ }^{23}$ Informally, we can find a positive element below $\mathbf{c}$ which is the intersection of some $\mathbf{a} \in \mathfrak{B}_{\mathfrak{a}}^{+}$, some number of $\mathbf{c}_{\gamma, \epsilon}$ 's which appear positively, and some number of $\mathbf{c}_{\gamma, \epsilon}$ 's which appear negatively, but remembering that each $\left\{\mathbf{c}_{\gamma, \epsilon}: \epsilon<\mu_{\gamma}\right\}$ is an antichain, among the elements which appear positively there can be no more than one from each antichain.

${ }^{24}$ Case 1. We connect to both $a_{\gamma_{1}}$ and $a_{\gamma_{2}}$ or neither, so there is no conflict. Case 2. The truth values are different but they collapse to different elements, so there is no conflict. Case 3. The truth values are different, they collapse to the same $\beta$ (and so, by (d), are contained in the same piece of the antichain for $\beta$ ). The c's in this case do not intersect, so there is no conflict.
} 
As $\mathbf{b}_{u} \in \mathcal{D}_{\mathfrak{a}}, \mathbf{b}_{u} \cap \mathbf{d}>0$ so we may assume without loss of generality that $\mathbf{d} \leq \mathbf{b}_{u}$. Enumerate $u=\left\langle\gamma_{i}: i<|u|\right\rangle$. Now as each $\left\langle\mathbf{a}_{\gamma_{i}, \epsilon}: \epsilon\left\langle\mu_{\gamma_{i}}\right\rangle\right.$ is a maximal antichain of $\mathfrak{B}_{\mathfrak{a}}$, we may choose by induction on $i$ a function $f$ with domain $u$ such that for each $i, f(i)<\mu_{\gamma_{i}}$ and $\mathbf{a}_{\gamma_{i}, f(i)} \cap \bigcap_{j<i} \mathbf{a}_{\gamma_{j}, f(j)} \cap \mathbf{d}>0$. Let $\mathbf{a}_{f}$ denote $\bigcap_{i<|u|} \mathbf{a}_{\gamma_{i}, f(i)}$. Since $\mathbf{d} \leq \mathbf{b}_{u}$, we know $\mathbf{a}_{f} \cap \mathbf{b}_{u}>0$, thus, in fact, $\mathbf{a}_{f} \leq \mathbf{b}_{u}$. [Why? By definition, $\mathbf{b}_{u}$ only depends on information about collisions, which remain constant on $\mathbf{a}_{f}$ by construction of our maximal antichains.]

Let $\mathbf{d}_{*}=\mathbf{d} \cap \mathbf{a}_{f}=\mathbf{d} \cap \mathbf{a}_{f} \cap \mathbf{b}_{u}>0$. Since $\mathbf{d}_{*} \leq \mathbf{a}_{f} \leq \mathbf{b}_{u}$ (and recall $u=\operatorname{dom}(f)$ ) for any $\gamma_{1}, \gamma_{2} \in u$ we have that $\gamma_{1} \neq \gamma_{2}$ implies either $\mathbf{t}\left(\gamma_{1}\right)=\mathbf{t}\left(\gamma_{2}\right)$ or $\beta_{\gamma_{1}, f\left(\gamma_{1}\right)} \neq$ $\beta_{\gamma_{2}, f\left(\gamma_{2}\right)}$. It follows that the equations in the definition of $\mathfrak{B}_{\mathfrak{b}}^{0}$ permit

$$
\mathbf{d}_{*} \cap\left\{\mathbf{c}_{\gamma, f(\gamma)}: \gamma \in u\right\}>0 .
$$

Now define $h: \mathcal{X} \rightarrow \mathfrak{B}_{\mathfrak{a}}$ to be the identity on $\mathfrak{B}_{\mathfrak{a}}, h\left(\mathbf{c}_{\gamma, f(\gamma)}\right)=\mathbf{d}_{*}$ for $\gamma \in u$, and $h\left(\mathbf{c}_{\gamma, \epsilon}\right)=0_{\mathfrak{B}_{\mathfrak{a}}}$ for $(\gamma, \epsilon) \notin\{(\gamma, f(\gamma)): \gamma \in u\}$. This $h$ extends to a homomorphism $\hat{h}$ from $\mathfrak{B}_{\mathfrak{b}}$ onto $\mathfrak{B}_{\mathfrak{a}}$ which is the identity on $\mathfrak{B}_{\mathfrak{a}}$ and sends $\mathbf{d}$ to a positive element. Thus, a suitable ultrafilter exists; fix one. This completes the construction of $\left(\mathfrak{B}_{\mathfrak{b}}, \mathcal{D}_{\mathfrak{b}}\right)$ and the verification that $\left\langle\mathbf{b}_{\gamma}^{1}: \gamma<\theta\right\rangle$ is a solution for $\overline{\mathbf{b}}$ there.

It remains to verify that the pair $\left(\mathfrak{B}_{\mathfrak{a}}, \mathfrak{B}_{\mathfrak{b}}\right)$ satisfies the $(\kappa, \mathcal{I}, \bar{m})$-pattern transfer property. Suppose we are given $\left\langle\mathbf{a}_{\alpha}^{2}: \alpha<\kappa\right\rangle$ a sequence of positive elements of $\mathfrak{B}_{\mathfrak{b}}$. Similarly to the earlier proof that $\mathfrak{B}_{\mathfrak{a}} \lessdot \mathfrak{B}_{\mathfrak{b}}$, as the generators are dense in the completion, we may find for each $\alpha<\kappa$ an $\mathbf{i}_{\alpha}=\left(\mathbf{x}_{\alpha}, u_{\alpha}, f_{\alpha}, v_{\alpha}, \bar{w}_{\alpha}=\left\langle w_{\alpha, \beta}: \beta \in\right.\right.$ $\left.\left.v_{\alpha}\right\rangle\right)$ such that $\mathbf{x}_{\alpha} \in \mathfrak{B}_{\mathfrak{a}}^{+}, u_{\alpha}, v_{\alpha}$ are finite subsets of $\theta, f_{\alpha}$ is a function with finite domain $u_{\alpha} \subseteq \theta$ such that $f_{\alpha}(\gamma)<\mu_{\gamma}$ for $\gamma \in u_{\alpha} ; w_{\alpha, \beta}$ is a finite subset of $\mu_{\beta}$ for $\beta \in v_{\alpha}$; we have that $\left\{\left(\gamma, f_{\alpha}(\gamma)\right): \gamma \in u_{\alpha}\right\} \cap\left\{(\delta, \epsilon): \delta \in v_{\alpha}, \epsilon \in w_{\alpha, \beta}\right\}=\emptyset$, and if $\gamma_{1} \neq \gamma_{2} \in u_{\alpha}, \mathbf{t}\left(\gamma_{1}\right) \neq \mathbf{t}\left(\gamma_{2}\right)$, then $\beta_{\gamma_{1}, f_{\alpha}\left(\gamma_{1}\right)} \neq \beta_{\gamma_{2}, f_{\alpha}\left(\gamma_{2}\right)}$; and together they satisfy

$$
\mathfrak{B}_{\mathfrak{b}} \models 0<\mathbf{x}_{\alpha} \cap \bigcap\left\{\mathbf{c}_{\gamma, f_{\alpha}(\gamma)}: \gamma \in u_{\alpha}\right\} \cap \bigcap\left\{-\mathbf{c}_{\beta, \epsilon}: \beta \in v_{\alpha}, \epsilon \in w_{\alpha, \beta}\right\} \leq \mathbf{a}_{\alpha}^{2}
$$

Next we will want to use the $\Delta$-system lemma to smooth out collisions. Towards this, define $B_{\alpha}=\left\{\beta_{\gamma, f(\gamma)}: \gamma \in u_{\alpha}\right\}$, and define $g_{\alpha}: B_{\alpha} \rightarrow\{0,1\}$ to be the function given by $\beta_{\gamma, f(\gamma)} \mapsto \mathbf{t}(\gamma)$ for $\gamma \in u_{\alpha}$. [We have to justify why this is a function: if $\gamma_{1} \neq \gamma_{2} \in u_{\alpha}, \mathbf{t}\left(\gamma_{1}\right) \neq \mathbf{t}\left(\gamma_{2}\right)$, then $\beta_{\gamma_{1}, f_{\alpha}\left(\gamma_{1}\right)} \neq \beta_{\gamma_{2}, f_{\alpha}\left(\gamma_{2}\right)}$ otherwise the intersection in equation (1) would be empty.]

Then, by the $\Delta$-system lemma, we may find $\mathcal{U} \in[\kappa]^{\kappa}$ along with $u_{*}, f_{*}, B_{*}, g_{*}$ satisfying:

- $\left\langle u_{\alpha}: \alpha \in \mathcal{U}\right\rangle$ is a $\Delta$-system with heart $u_{*}$.

- for each $\alpha \in u, f_{\alpha}\left\lceil u_{*}=f_{*}\right.$ (recall $f_{\alpha}(\gamma)$ can take fewer than $\kappa$ values).

- $\left\langle B_{\alpha}: \alpha \in \mathcal{U}\right\rangle$ is a $\Delta$-system with heart $B_{*}$.

- for each $\alpha \in u, g_{\alpha}\left\lceil B_{*}=g_{*}\right.$.

Let us show that for any finite $u \subseteq \mathcal{U}$, letting $w=\bigcup_{\alpha \in u} u_{\alpha}$, we have that

$$
\bigcap_{\gamma \in w} \mathbf{c}_{\gamma, f_{\alpha}(\gamma)}=\bigcap\left\{\mathbf{c}_{\gamma, f_{\alpha}(\gamma)}: \alpha \in u, \gamma \in u_{\alpha}\right\}>0 .
$$

Why? It suffices to show that if $\alpha_{1}, \alpha_{2} \in u, \gamma_{1} \in u_{\alpha_{1}}, \gamma_{2} \in u_{\alpha_{2}}$, then $\mathbf{c}_{\gamma_{1}, f_{\alpha_{1}}}\left(\gamma_{1}\right) \cap$ $\mathbf{c}_{\gamma_{2}, f_{\alpha_{2}}}\left(\gamma_{2}\right)>0$. First suppose $\alpha_{1}=\alpha_{2}$; then this follows from equation (II). Next, suppose $\alpha_{1} \neq \alpha_{2}$ but $\gamma_{1}=\gamma_{2}$. This can happen only if $\gamma:=\gamma_{1}=\gamma_{2}$ belongs to $u_{*}$, in which case $f_{\alpha_{1}}(\gamma)=f_{*}(\gamma)=f_{\alpha_{2}}(\gamma)$ and there is no problem from equation (ii). Moreover, necessarily $\beta_{\gamma, f_{\alpha_{1}}}(\gamma)=\beta_{\gamma, f_{\alpha_{2}}}(\gamma)$, so this value, call it $\beta$, must belong 
to $B_{*}$ and $g_{\alpha_{1}}(\beta)=g_{*}(\beta)=g_{\alpha_{2}}(\beta)$ thus $\mathbf{t}\left(\gamma_{1}\right)=\mathbf{t}\left(\gamma_{2}\right)$ and there is no problem from (iii). Finally, suppose $\alpha_{1} \neq \alpha_{2}$ and $\gamma_{1} \neq \gamma_{2}$. Equation (ii) is irrelevant. As before, if $\beta_{\gamma_{1}, f_{\alpha_{1}}}\left(\gamma_{1}\right)=\beta_{\gamma_{2}, f_{\alpha_{2}}}\left(\gamma_{2}\right)$, then this value, call it $\beta$, must belong to $B_{*}$ and $g_{\alpha_{1}}(\beta)=g_{*}(\beta)=g_{\alpha_{2}}(\beta)$ thus $\mathbf{t}\left(\gamma_{1}\right)=\mathbf{t}\left(\gamma_{2}\right)$ and there is again no problem from equation (iii). This completes the proof of (m).

Just as in 10.19(4), this tells us that for a finite $u \subseteq \mathcal{U}$, if $\bigcap_{\alpha \in u} \mathbf{x}>0$, then

$$
0<\bigcap_{\alpha \in u} \mathbf{x}_{\alpha} \cap \bigcap\left\{\mathbf{c}_{\gamma, f_{\alpha}(\gamma)}: \gamma \in u_{\alpha}\right\} \cap \bigcap\left\{-\mathbf{c}_{\beta, \epsilon}: \beta \in v_{\alpha}, \epsilon \in w_{\alpha, \beta}\right\} .
$$

Recall that by 8.20 , this suffices for pattern transfer, so finishes the proof.

Recall that $\kappa$ is a regular uncountable cardinal and $\lambda \geq \kappa$.

Conclusion 10.25. There exists a regular ultrafilter $\mathcal{D}$ on $\lambda$ such that:

(1) $\mathcal{D}$ is $\kappa$-good, i.e. $(\mathbb{N},<)^{\lambda} / \mathcal{D}$ is $\kappa$-saturated 25

(2) if $\mathfrak{m} \in \mathcal{M}$ then $\mathcal{D}$ is $\left(\lambda^{+}, T_{\mathfrak{m}}\right)$-good.

(3) if $\mathfrak{n} \in \mathcal{N}$ then $\mathcal{D}$ is not $\left(\kappa^{+}, T_{\mathfrak{n}}\right)$-good.

Proof. Let $\left\langle S_{\gamma}: \gamma\left\langle 2^{\lambda}\right\rangle\right.$ partition $2^{\lambda}$ into sets each of cardinality $2^{\lambda}$ with $\gamma \leq$ $\min \left(S_{\gamma}\right)$. We choose by induction on $\alpha \leq 2^{\lambda}$ not only $\mathfrak{b}_{\alpha}$ but also $\bar{f}_{\alpha}$ such that:

(1) $\overline{\mathfrak{b}}=\left\langle\mathfrak{b}_{\alpha}: \alpha \leq 2^{\lambda}\right\rangle$ is a $\leq_{\mathrm{AP}}$-increasing continuous general construction sequence, so by definition and our work above this will mean:

(a) $\mathfrak{b}_{0}=\mathfrak{a}_{*}$,

(b) each $\mathfrak{b}_{\alpha} \in \mathrm{AP}$,

(c) $\alpha<\beta \Longrightarrow\left(\mathfrak{B}_{\mathfrak{a}}, \mathfrak{B}_{\mathfrak{b}}\right)$ satisfies the $(\kappa, \mathcal{I}, \bar{m})$-pattern transfer property,

(d) each $\left|\mathfrak{B}_{\mathfrak{b}_{\alpha}}\right| \leq 2^{\lambda}$,

(e) each $\mathfrak{B}_{\mathfrak{b}_{\alpha}}$ satisfies the $(\kappa, \mathcal{I}, \bar{m})$-c.c.

(2) $\bar{f}_{\alpha}=\left\langle f_{\alpha, \gamma}: \gamma \in S_{\alpha}\right\rangle$

(3) $\bar{f}_{\alpha}$ lists $\mathcal{F}_{\alpha}=\left\{f: f\right.$ is a function from $[\theta]<\aleph_{0}$ into $\mathcal{D}_{\mathfrak{b}_{\alpha}}$ which is monotone for some $\left.\theta=\theta_{f} \leq \lambda\right\}$.

(4) Now if $\alpha=\gamma+1$ and $\gamma \in S_{\beta}$, so $\beta \leq \gamma$, and $\operatorname{range}\left(f_{\alpha, \gamma}\right) \subseteq \mathcal{D}_{\mathfrak{b}_{\gamma}}$, and if maintaining the restriction in (1) we can choose $\mathfrak{b}_{\alpha}$ such that $f_{\alpha, \gamma}$ has a multiplicative refinement $g_{\gamma}$ with range $\subseteq \mathcal{D}_{\mathfrak{b}_{\alpha}}$, then there is such a refinement [i.e., then we do so]. Otherwise, we do nothing.

Alternately, in this step we could say that if $f_{\alpha, \gamma}$ is a possibility pattern for a theory $T=T_{f_{\alpha, \gamma}}$ such that one of the following occurs:

- $\theta_{f_{\alpha, \gamma}}<\kappa$,

- $T_{f_{\alpha, \gamma}}$ is $T_{\mathfrak{m}}$ for $\mathfrak{m} \in \mathcal{M}$, and the possibility pattern in question comes from a positive $R$-type together with a single formula of the form $Q_{\nu_{*}}(x)$ for some $\nu_{*} \in \mathcal{T}_{1}$ (or: together with a single formula of the form $P_{\eta_{*}}(y)$ for some $\left.\eta_{*} \in \mathcal{T}_{2}\right)$

- $T_{f_{\alpha, \gamma}}$ is $T_{\mathrm{rg}}$ and the possibility pattern comes from a type in positive and negative instances of $R$.

then we solve it using 10.20, 10.22, or 10.24, otherwise we do nothing. Note that 10.20, 10.22, 10.24 show that at the very least, these three kinds of types will be handled if we take the first alternative.

\footnotetext{
${ }^{25}$ Equivalently, for any model $M$ with $\left|\tau_{M}\right|<\kappa, M^{\lambda} / \mathcal{D}$ is $\kappa$-saturated.
} 
Having built our Boolean algebra and ultrafilter, we choose the data of separation of variables $\mathcal{D}_{0}, \mathbf{j}$ to go with this $\mathfrak{B}_{\mathfrak{a}}$ and $\mathcal{D}_{\mathfrak{a}}$ in the sense of 7.1, which gives us the regular ultrafilter desired, recalling Theorem 7.3. Note that by our analysis of ultrapower types in 5.6. these $R$-types suffice for saturation for $T_{\mathfrak{m}}$, and by 9.4 the fact that our Boolean algebra $\mathfrak{B}_{\mathfrak{a}}$ has the $(\kappa, \mathcal{I}, \bar{m})$-c.c. suffices for non-saturation of $T_{\mathfrak{n}}$ for $\mathfrak{n} \in \mathcal{N}$.

\section{MAin RESUlts AND THE BIG PICTURE}

In this section we summarize the main consequences of our construction for Keisler's order.

Recall that we can easily form new countable theories as the "disjoint union," or sum, of up to countably many countable theories. More formally:

Definition 11.1. Recall $T=\sum T_{n}$ when without loss of generality $\left\langle\tau\left(T_{n}\right): n<\omega\right\rangle$ are pairwise disjoint and have only predicates, and we have countably many new unary predicates $\left\{P_{n}: n<\omega\right\}$ with $\left\{P_{n}: n<\omega\right\} \cap \tau\left(T_{n}\right)=\emptyset$ for each $n$. Then $M \models T$ iff $\left\langle P_{n}^{M}: n<\omega\right\rangle$ are pairwise disjoint and $\left(M \uparrow P_{n}^{M}\right) \uparrow \tau\left(T_{n}\right) \models T_{n}$, and if $R \in \tau\left(T_{n}\right)$ then $R^{M} \subseteq{ }^{\operatorname{arity}(R)}\left(P_{n}^{M}\right)$.

In this notation:

Corollary 11.2. Continuing with the objects of 10.25, given any $\mathfrak{m}_{\ell} \in \mathcal{M}$ for $\ell<\omega$, we have that $T_{*}=\sum_{\ell<\omega} T_{\mathfrak{m}_{\ell}}$ is a complete countable simple theory, and $\mathcal{D}$ is $\left(\lambda^{+}, T_{*}\right)$-good.

Recall that $u \in[X]^{\leq \aleph_{0}}$ means that $u$ is an at most countable subset of $X$. Given any fast sequence and a family of independent level functions, our construction above gives a set of continuum many theories which can be thought of as Keislerindependent in the following strong sense. Suppose from the basic theories we form all possible "small composite theories" (countable, complete theories formed in the natural way as disjoint unions of at most countably many theories from our original set). Then our construction has shown that even the composite theories interact as freely as possible in the sense of Keisler's order, reflecting only the interaction in their indices, as the next theorem makes precise.

Theorem 11.3. We can find $\bar{T}$ such that:

(1) $\bar{T}=\left\langle T_{u}: u \in\left[2^{\aleph_{0}}\right] \leq \aleph_{0}\right\rangle$

(2) $T_{u}$ is a complete first order countable simple theory with trivial forking

(3) $T_{u} \unlhd T_{v}$ if and only if $u \subseteq v$, for $u, v \in\left[2^{\aleph_{0}}\right] \leq \aleph_{0}$.

(4) if $W \subseteq 2^{\aleph_{0}}, \aleph_{0} \leq \mu<\mu^{+} \leq \kappa=\operatorname{cof}(\kappa) \leq \lambda$ then there is a regular ultrafilter $\mathcal{D}$ on $\lambda$ such that:

(a) $\mathcal{D}$ is $\kappa$-good

(b) if $u \in[W] \leq \aleph_{0}$ then $\mathcal{D}$ is $\left(\lambda^{+}, T_{u}\right)$-good

(c) if $u \in\left[2^{\aleph_{0}}\right] \leq \aleph_{0}, u \nsubseteq W$ then $\mathcal{D}$ is not $\left(\kappa^{+}, T_{u}\right)$-good.

Proof. Let $\bar{m}$ be a fast sequence, let $\bar{E}$ and $\Xi$ satisfy the hypotheses of 6.22 , and let $\mathbf{M}_{*}=\left\{\mathfrak{m}_{\alpha}=\operatorname{par}\left[\bar{m}, \bar{E}, \xi_{\alpha}\right]: \alpha<2^{\aleph_{0}}\right\}$ be as defined in [6.22, Let $W$ play the role of $\mathcal{M} \subseteq \mathbf{M}_{*}$. Then by our construction there is a regular ultrafilter $\mathcal{D}$ on any infinite $\lambda$ as in (4), satisfying also (4)(a), such that $\mathcal{D}$ is good for every $T_{\mathfrak{m}}, \mathfrak{m} \in \mathcal{M}$, 
and $\mathcal{D}$ is not good for any $T_{\mathfrak{n}}, \mathfrak{n} \in \mathbf{M}_{*} \backslash \mathcal{M}$. Note that any sum $T_{u}$ of theories $\left\{T_{\mathfrak{m}_{\alpha}}: \alpha \in u\right\}$ is complete and remains simple with trivial forking. Clearly (3) and (4)(b), (c) follow.

As immediate consequences, we obtain several "more quotable" theorems.

Theorem 11.4. There exist a perfect set of incomparable theories in Keisler's order, in ZFC, already within the class of countable simple unstable theories whose only forking comes from equality.

Proof. Use any set of countably many pairwise incomparable theories from Theorem 11.3 to label the nodes of an infinite binary branching tree, and consider the set of theories which correspond to disjoint unions of theories along a given branch.

Theorem 11.5. In the (countable, complete) simple non low theories,

(1) there is a chain of cardinality continuum, and

(2) there is an antichain of cardinality continuum

in Keisler's order.

Proof. Enumerate a countable subset of the theories from Theorem 11.3 as $\left\langle T_{q}\right.$ : $q \in \mathbb{Q}\rangle$. Let $\left\langle C_{\beta}=\left(C_{\beta}^{0}, C_{\beta}^{1}\right): \beta<2^{\aleph_{0}}\right\rangle$ enumerate the continuum many cuts of the rationals. Let $T_{\beta}$ be the theory which is the disjoint union of $\left\{T_{q}: q \in C_{\beta}^{0}\right\}$. Then clearly $\alpha \leq \beta$ implies $T_{\alpha} \unlhd T_{\beta}$. As the rationals are dense in the reals, and our family of theories are pairwise incomparable, if $\alpha<\beta$ then $T_{\alpha} \triangleleft T_{\beta}$. This gives us a chain of cardinality continuum.

For an antichain of cardinality $\mathfrak{c}$, use all the theories from Theorem 11.3.

Remark 11.6. Even in this frame the theories involved seem simple.

Theorem 11.7. There exist continuum many complete, countable, simple theories whose only forking comes from equality, and which are pairwise incomparable in Keisler's order.

Proof. Consider all $u$ of size 1 in Theorem 11.3, i.e., in the notation of that proof, consider $\left\{T_{\mathfrak{m}_{\alpha}}: \alpha<2^{\aleph_{0}}\right\}$.

Conclusion 11.8. Keisler's order is not at all simple, indeed the partial order on the family of subsets of $\mathbb{N}$ is embeddable into it.

In fact, with more work we can upgrade 11.8 to the following a priori much stronger result. We include the proof in the next section.

Theorem 11.9. $\mathcal{P}(\omega)$ / fin embeds into Keisler's order.

Proof. See page 69, below.

The theorem whose proof concludes the next section explains:

Theorem 11.10. There is a family of parameters $\{\mathfrak{m}[A]: A \subseteq \omega\}$ such that each $T_{\mathfrak{m}[A]}$ is countable, complete, simple, and low, and the following are equivalent for any $\lambda \geq 2^{\aleph_{0}}$ and any set $\mathcal{X} \subseteq \mathcal{P}(\omega)$ :

(1) There exists a regular ultrafilter $\mathcal{D}$ on $\lambda$ such that $\mathcal{X}=\{A \subseteq \omega: \mathcal{D}$ is $\left(\lambda^{+}, T_{\mathfrak{m}[A]}\right)$-good $\}$.

(2) $\mathcal{X} \supseteq[\omega]^{<\aleph_{0}}$ is an ideal. 
Proof. See page 69, below.

Discussion 11.11. In light of our main theorems, it would be natural to consider strengthenings of Keisler's order, for instance, by restricting consideration to ultrafilters on $\lambda$ which are $\lambda$-good [but of course not necessarily $\lambda^{+}$-good]. However, our construction can be carried out even under such a definition, as we now spell out.

Definition 11.12. Let the strong Keisler order mean that $T_{1} \unlhd^{s} T_{2}$ if and only if for every infinite $\lambda$, for every regular ultrafilter on $\lambda$ which is $\lambda$-good, for every $M_{1} \models T_{1}$, and every $M_{2} \models T_{2}$, if $\left(M_{2}\right)^{\lambda} / \mathcal{D}$ is $\lambda^{+}$-good, then $\left(M_{1}\right)^{\lambda} / \mathcal{D}$ is $\lambda^{+}$-good.

Theorem 11.13. There exists a family $\left\{\mathcal{T}_{\mathfrak{m}_{\alpha}}: \alpha<2^{\aleph_{0}}\right\}$ of continuum many complete countable simple theories, with forking coming only from equality, such that for any $u, v \subseteq 2^{\aleph_{0}}$ and $u, v$ and most countable, letting $T_{u}, T_{v}$ denote the "disjoint unions" of $\left\{T_{\mathfrak{m}_{\alpha}}: \alpha \in u\right\},\left\{T_{\mathfrak{m}_{\beta}}: \beta \in v\right\}$ respectively, we have that $T_{u} \unlhd^{s} T_{v}$ in the strong Keisler order if and only if $u \subseteq v$.

Proof. This is a simplified version of Theorem 11.3 in the case $\mu<\mu^{+}=\kappa=\lambda$.

Remark 11.14. Note that Theorem 11.13 deals with the case where we quantify over all $\lambda$. We could ask the analogous question $\unlhd_{\lambda}^{s}$, restricting to ultrafilters on $\lambda$. The proof of 11.13 shows that 11.13 remains true for $\unlhd_{\lambda}^{s}$ when $\lambda$ is any successor. If $\lambda$ is a regular limit, the construction should likewise go through using $\lambda=\kappa$ and Definition 8.8. If $\lambda$ is a singular limit cardinal, then any $\lambda$-good ultrafilter is $\lambda^{+}$-good. [This is a consequence of the fact that $T h(\mathbb{Q},<)$ is maximal in Keisler's order, see [33] IV.2.6. p. 337.] Hence there is only one class under $\unlhd_{\lambda}^{s}$.

Since we know that Keisler's order reduces to the study of $\varphi$-types, it was asked:

Earlier Problem. Determine whether there exists a function associating to each complete countable theory $T$ a formula $\varphi_{T}$ of the language of $T$ such that for any infinite $\lambda$, any regular ultrafilter $\mathcal{D}$ on $\lambda$, and any model $M \models T$, the ultrapower $M^{\lambda} / \mathcal{D}$ is $\lambda^{+}$-saturated if and only if it is $\lambda^{+}$-saturated for $\varphi_{T}$-types.

Call such a function an assignment of formulas to theories witnessing Keisler's order. The results of the present paper show no formula assignment can exist:

Corollary 11.15. There cannot exist an assignment of formulas to theories witnessing Keisler's order.

Proof. Continuing in the notation of Theorem 11.3, let $\left\langle\mathfrak{m}_{n}: n\langle\omega\rangle\right.$ be any countable sequence of distinct elements of $\mathcal{M}$ and let $T=\sum T_{\mathfrak{m}_{n}}$ be their disjoint union. Following 11.1, we may assume that without loss of generality $\left\{\tau\left(T_{\mathfrak{m}_{n}}\right): n<\omega\right\}$ are pairwise disjoint and have only predicates. Assume for a contradiction that there exists a formula $\varphi_{T}$ such that for any infinite $\lambda$, any regular ultrafilter $\mathcal{D}$ on $\lambda$, and any model $M \models T$, we would have $M^{\lambda} / \mathcal{D}$ is $\lambda^{+}$-saturated if and only if it is $\lambda^{+}$-saturated for $\varphi_{T}$-types. Fix $M$. The formula $\varphi_{T}$, being finite, is a formula of the language of $\bigcup\left\{\tau_{T_{\mathfrak{m}_{n}}}: n \in v\right\}$ for some finite $v \subseteq \omega$. By Theorem 11.3, there is a regular ultrafilter $\mathcal{D}$ on any uncountable $\lambda$ which is $\lambda^{+}$-good for $\left\{T_{\mathfrak{m}_{n}}: n \in v\right\}$ and not $\lambda^{+}$-good for $\left\{T_{\mathfrak{m}_{\ell}}: \ell \in \omega \backslash v\right\}$. So in $M^{\lambda} / \mathcal{D}$ we realize all $\varphi_{T^{-}}$-types over sets of size $\lambda$, but omit some other $\psi$-type over some set of size $\lambda$.

Discussion 11.16. However, it may be interesting to give a model-theoretic characterization of the theories $T$ for which such $a \varphi_{T}$ exists. This includes all stable 
theories (choose any formula with the finite cover property if one exists, if not choose any formula [33] VI.5.2), and all theories with $\mathrm{SOP}_{2}$ [21].

\section{EMBedDing $\mathcal{P}(\omega) /$ fin}

In this section we prove Theorem 11.9, Keisler's order embeds $\mathcal{P}(\omega) /$ fin, and Theorem 11.10 .

Convention 12.1. For this section, fix some fast sequence $\bar{m}$, and a sequence of graphs $\bar{E}$ which is good for it, just as in 6.12 above.

Convention 12.2. In this section, for each $A \subseteq \omega$, let $\mathfrak{m}[A]$ be the parameter $\operatorname{par}[\bar{m}, \bar{E}, \xi]$ where $\xi: \omega \rightarrow\{0,1\}$ satisfies $\xi^{-1}\{1\}=A$. Let $T_{\mathfrak{m}[A]}$ be its associated theory.

Our proof will show that $T_{\mathfrak{m}[A]} \unlhd T_{\mathfrak{m}[B]}$ if and only if $A \subseteq^{*} B$. The proof will also show this for the interpretability order $\unlhd^{*}$, not only Keisler's order $\unlhd$. [A recent introduction to $\unlhd^{*}$ is in $24, \S 1$. The equivalence relation induced by $\unlhd^{*}$ is strictly finer than that induced by $\unlhd$, see [24 2.12.]

It will be useful to make explicit a property of parameters which held in our main case above. 26 Note that 12.3 (1)(c) says that we can write, for each level $k$, a graph $\mathcal{S}_{k}$ such that the connections in $\mathcal{R}$ depend only on these graphs.

\section{Definition 12.3.}

(1) We say $\mathfrak{m}$ is forgetful when

(a) $\mathfrak{m}$ is self-dual for transparency

(b) for each $\mathbf{i}=1,2$ and $k$ there is $m_{k}^{\mathbf{i}}$ such that for all $\eta \in \mathcal{T}_{\mathbf{i}, k}$, $(\forall j)\left(\eta^{\curlyvee}\langle j\rangle \in \mathcal{T}_{\mathbf{i}, k+1} \Longleftrightarrow j<m_{k}^{\mathbf{i}}\right)$

(c) for each $k$ there is $S_{k} \subseteq m_{k}^{1} \times m_{k}^{2}$ such that if $(\eta, \nu) \in \mathcal{T}_{1, k} \times \mathcal{T}_{2, k}$ then $(\eta, \nu) \in \mathcal{R}_{k}^{\mathfrak{m}}$ if and only if $(\forall \ell<k)(\eta(\ell), \nu(\ell)) \in S_{\ell}$.

(2) So if $\mathfrak{m}$ is forgetful and $k<\omega$, then $\mathfrak{n}=\mathfrak{m}\lceil\geq k$ satisfies: $m_{n}^{\mathbf{i}}[\mathfrak{n}]=m_{n+k}^{\mathbf{i}}[\mathfrak{m}]$, and this does not depend on $\mathbf{i}$

$\mathcal{T}_{\mathbf{i}, n}[\mathfrak{n}]=\prod_{i<n} m_{k+i}^{\mathbf{i}}[\mathfrak{m}]$, and

$S_{n}[\mathfrak{n}]=S_{k+n}[\mathfrak{m}]$, which are each symmetric.

Informally, all nodes at level $k$ have the same number of immediate successors, and when extending edges to the next level (which recall only happens between elements whose restrictions are connected at all earlier levels) only the last value matters:

Remark 12.4. 12.3 $(1)(b)$ implies that if we are given $\eta \in \mathcal{T}_{1, k}$ and $\rho \in \mathcal{T}_{2, k}$ and $\eta^{\prime} \in \mathcal{T}_{1, k}$ and $\rho^{\prime} \in \mathcal{T}_{2, k}$, and $(\eta, \rho) \in \mathcal{R}$ and $\left(\eta^{\prime}, \rho^{\prime}\right) \in \mathcal{R}$, then for any $i<m_{k+1}^{1}$ and $j<m_{k+1}^{2}$,

$$
\left(\eta^{\frown}\langle i\rangle, \rho^{\frown}\langle j\rangle\right) \in \mathcal{R} \Longleftrightarrow\left(\eta^{\prime} \frown\langle i\rangle, \rho^{\prime} \frown\langle j\rangle\right) \in \mathcal{R}
$$

So the parameter depends in the natural way on a sequence of graphs. The parameters used in the main arguments above satisfied this definition. The following easy consequence will also be useful.

\footnotetext{
${ }^{26}$ In Definition 12.3 whether $\left(\eta^{\frown}\left\langle\ell_{1}\right\rangle, \rho^{\frown}\left\langle\ell_{2}\right\rangle\right)$ is an edge in $\mathcal{R}$ depends only on the fact that $(\eta, \rho) \in \mathcal{R}$ and on $\ell_{1}, \ell_{2}$. There is no "memory" coming from the path in the tree which affects the graph used at this stage (just the level matters).

${ }^{27}$ so we don't really need $m^{1}$ and $m^{2}$ in item (b).
} 
Claim 12.5. Suppose $\mathfrak{m}$ is a forgetful parameter, $\eta_{*}=\eta_{0}{ }^{\wedge}\langle i\rangle^{\wedge} \eta_{\infty} \in \lim \left(\mathcal{T}_{1}^{\mathfrak{m}}\right)$, and $\rho_{*}=\rho_{0} \bumpeq\langle j\rangle^{\wedge} \rho_{\infty} \in \lim \left(\mathcal{T}_{2}^{\mathfrak{m}}\right)$, where $\operatorname{lgn}\left(\eta_{0}\right)=\operatorname{lgn}\left(\rho_{0}\right)<\omega$. Suppose that for every $s<\omega,\left(\eta_{*}\left\lceil s, \rho_{*}\lceil s) \in \mathcal{R}^{\mathfrak{m}}\right.\right.$. If we replace $i, j$ by $i^{\prime}, j^{\prime}$ respectively so that $\left(\eta_{0} \frown\left\langle i^{\prime}\right\rangle, \rho_{0} \bumpeq\left\langle j^{\prime}\right\rangle\right) \in \mathcal{R}^{\mathfrak{m}}$, then it remains true that for every $s<\omega$,

$$
\left.\left(\left(\eta_{0} \wedge\left\langle i^{\prime}\right\rangle\right\urcorner \eta_{\infty}\right)\left\lceil s,\left(\rho_{0} \bumpeq\left\langle j^{\prime}\right\rangle\right\urcorner \rho_{\infty}\right) \uparrow s\right) \in \mathcal{R}^{\mathfrak{m}} .
$$

Proof. By definition of forgetful.

Next, let $\mathfrak{m}$ be any forgetful parameter. We define a useful class of restricted models, starting with the simplest case.

Definition 12.6. For any $\mathfrak{m}$ and $M \models T_{\mathfrak{m}}^{0}, k<\omega$, and $(\eta, \rho) \in \mathcal{T}_{1, k}^{\mathfrak{m}} \times \mathcal{T}_{2, k}^{\mathfrak{m}}$,

(A) we define $\mathfrak{n}=\mathfrak{m} \uparrow(\eta, \rho)$ by:

(a) $\mathcal{T}_{1}^{\mathfrak{n}}=\left\{\nu: \eta^{\curvearrowright} \nu \in \mathcal{T}_{1}^{\mathfrak{m}}\right\}$

(b) $\mathcal{T}_{2}^{\mathfrak{n}}=\left\{\nu: \rho^{\wedge} \nu \in \mathcal{T}_{2}^{\mathfrak{m}}\right\}$

(c) $\mathcal{R}_{k}^{\mathfrak{n}}=\left\{\left(\nu_{1}, \nu_{2}\right):\left(\eta^{\wedge} \nu_{1}, \rho^{\wedge} \nu_{2}\right) \in \mathcal{R}_{k}^{\mathfrak{m}}\right\}$

(B) we define $N=N[\eta, \rho, M]$ as the following $\tau\left(T_{\mathfrak{n}}\right)$-model:

(a) the universe is $Q_{\eta}^{M} \cup P_{\rho}^{M}$.

(b) $Q_{\nu}^{N}=Q_{\eta^{\urcorner} \nu}^{M}$ for $\eta^{\urcorner} \nu \in \mathcal{T}_{1}^{\mathfrak{m}}$

(c) $P_{\nu}^{N}=P_{\rho^{\wedge} \nu}^{M}$ for $\rho^{\wedge} \nu \in \mathcal{T}_{2}^{\mathfrak{m}}$

(d) $\mathcal{R}^{N}=\mathcal{R}^{M}\left\lceil\left(Q_{\eta}^{M} \times P_{\rho}^{M}\right)\right.$.

Claim 12.7. Assume $\mathfrak{n}=\mathfrak{m} \uparrow(\eta, \rho)$ where $(\eta, \rho) \in \mathcal{R}_{k}^{\mathfrak{m}}$, and $N=N[\eta, \rho, M]$. Then:

(1) If $M \models T_{\mathrm{m}}^{0}$, then $N \models T_{\mathrm{n}}^{0}$.

(2) If $M \models T_{\mathfrak{m}}$, then:

(a) $N \models T_{\mathfrak{n}}$, and

(b) if $A \subseteq N$ and $\bar{a}, \bar{b} \in{ }^{n} N$ then $\operatorname{tp}(\bar{a}, A, M)=\operatorname{tp}(\bar{b}, A, M)$ if and only if $\operatorname{tp}(\bar{a}, A, N)=\operatorname{tp}(\bar{b}, A, N)$.

(3) If $M$ is a $\kappa$-saturated model of $T_{\mathfrak{m}}$, then $N$ is a $\kappa$-saturated model of $T_{\mathfrak{n}}$.

Proof. (1) is by the definitions. (2) (a) is easy. (2)(b) is by the elimination of quantifiers. (3) follows from (2)(b). (Or see 12.12 below.)

Claim 12.8. Assume $\mathfrak{m}$ is forgetful and $k<\omega$.

(Claim) If $(\eta, \rho) \in \mathcal{R}_{\mathfrak{m}, k}$ and $\left(\eta^{\prime}, \rho^{\prime}\right) \in \mathcal{R}_{\mathfrak{m}, k}$ then $\mathfrak{m}\left\lceil(\eta, \rho)=\mathfrak{m}\left\lceil\left(\eta^{\prime}, \rho^{\prime}\right)\right.\right.$.

(Defn) Let $\mathfrak{n}=\mathfrak{m}\left\lceil\geq k\right.$ be $\mathfrak{m}\left\lceil(\eta, \rho)\right.$ for any (some) $(\eta, \rho) \in \mathcal{R}_{\mathfrak{m}, k}$.

Proof. By the definition of forgetful.

Now we generalize 12.6, but there is a subtlety. Suppose we are given $M \models T_{\mathfrak{n}}$ (or just $M \models T_{\mathfrak{n}}^{0}$ ) and $k<\omega$, and non-empty $\Lambda_{1} \subseteq \mathcal{T}_{1, k}, \Lambda_{2} \subseteq \mathcal{T}_{2, k}$ such that $\Lambda_{1} \times \Lambda_{2} \subseteq \mathcal{R}$ (i.e. a complete bipartite graph in the template at level $k$ ). We define $N=N\left[\Lambda_{1}, \Lambda_{2}, M\right]$ not by looking at the multi-rooted subtrees of $\mathcal{T}_{1}, \mathcal{T}_{2}$ which start in $\Lambda_{1}$ or $\Lambda_{2}$ respectively, and then restricting $M$ to predicates coming from these multi-rooted subtrees in the natural sense. Rather, we form a new model "by gluing" (or "by stacking on top of") all the subtrees with roots in $\Lambda_{1}$ to make the rooted tree on the left, and stacking up all the subtrees with roots in $\Lambda_{2}$ to make the rooted tree on the right. So $Q_{\langle\rangle}^{N}$ is the union $\bigcup\left\{Q_{\eta}^{M}: \eta \in \Lambda_{1}\right\}$ and $P_{\langle\rangle}^{N}$ is the union $\bigcup\left\{P_{\rho}^{M}: \rho \in \Lambda_{2}\right\}$. In general, $Q_{\nu}^{N}$ is the union of $\bigcup\left\{Q_{\eta^{\prime} \nu}^{M}: \eta \in \Lambda_{1}\right\}$, and $P_{\nu}^{N}$ is the union of $\bigcup\left\{P_{\rho^{\prime} \nu}^{M}: \rho \in \Lambda_{2}\right\}$. The edges in the model stay the same: 
$R^{N}=R^{M} \mid Q_{\langle\rangle}^{N} \times P_{\langle\rangle}^{N}$ [note: roman $R$ in the model, not $\mathcal{R}$ in the template; for more, see 12.12. This works well because $\mathfrak{m}$ is forgetful, so the subtrees sit exactly on top of each other, with the same branching and the same $\mathcal{R}$-information.

We will be most interested in cases of the form $N=N[\{\eta\}, \Lambda, M]$ where $\eta$ is a singleton and $\Lambda=\left\{\rho \in \mathcal{T}_{2}:(\eta, \rho) \in \mathcal{R}\right\}$ is the set of all neighbors of $\eta$, or the parallel $N=N[\Lambda,\{\rho\}, M]$, though we give the general definition.

Definition 12.9. Assume $\mathfrak{m}$ is forgetful, $M \models T_{\mathfrak{m}}, k<\omega, \emptyset \neq \Lambda_{\ell} \subseteq \mathcal{T}_{\ell, k}^{\mathfrak{m}}$ for $\ell=1,2$ and $\Lambda_{1} \times \Lambda_{2} \subseteq \mathcal{R}_{k}^{\mathfrak{m}}$.

We define $N=N\left[\Lambda_{1}, \Lambda_{2}, M\right]$ as the following $\tau(\mathfrak{m}\lceil\geq k)$-model:

- universe: $\bigcup\left\{Q_{\eta}^{M} \cup P_{\rho}^{M}:(\eta, \rho) \in \Lambda_{1} \times \Lambda_{2}\right\}$

- $Q_{\nu}^{N}=\bigcup\left\{Q_{\eta\urcorner \nu}^{M}: \eta \in \Lambda_{1}\right\}$, so in particular, $\mathcal{Q}^{N}=Q_{\langle\rangle}^{N}$.

- $P_{\nu}^{N}=\bigcup\left\{P_{\eta^{-} \nu}^{M}: \eta \in \Lambda_{1}\right\}$, so in particular, $\mathcal{P}^{N}=P_{\langle\rangle}^{N}$.

- $R^{N}=R^{M}\left\lceil\mathcal{Q}^{N} \times \mathcal{P}^{N}\right.$.

Note that 12.9 gives an interpretation of $N$ in $M$. Since we will mainly use the case where either $\Lambda_{1}$ or $\Lambda_{2}$ has cardinality 1 , we spell out this special case.

Observation 12.10. Let $\mathfrak{m}$ be a forgetful parameter. For $M \models T_{\mathfrak{m}}$,

(1) if $\Lambda_{1}=\{\eta\}$ and $\Lambda_{2} \subseteq\left\{\rho \in \mathcal{T}_{2, k}:(\eta, \rho) \in \mathcal{R}\right\}$, then our model has

- universe: $Q_{\eta}^{M} \cup \bigcup\left\{P_{\rho}^{M}: \rho \in \Lambda_{2}\right\}$

- $Q_{\nu}^{N}=Q_{\eta^{-} \nu}^{M}$.

- $P_{\nu}^{N}=\bigcup\left\{P_{\rho>\nu}^{M}: \rho \in \Lambda\right\}$.

- $R^{N}=R^{M}\left\lceil\mathcal{Q}^{N} \times \mathcal{P}^{N}\right.$, i.e. we retain all existing edges between elements which make it into the domain of the new model.

(2) We have the parallel in the case where $\Lambda_{2}=\{\rho\}$ and $\Lambda_{1} \subseteq\left\{\eta \in \mathcal{T}_{1, k}\right.$ : $(\eta, \rho) \in \mathcal{R}\}$.

Convention 12.11. We may write $N[\eta, \Lambda, M]$ instead of $N[\{\eta\}, \Lambda, M]$ and likewise we may write $N[\Lambda, \rho, M]$ instead of $N[\Lambda,\{\rho\}, M]$.

Comparing Definition 12.6 and Definition 12.9, the reader will notice that in 12.9 we don't define a new parameter $\mathfrak{n}=\mathfrak{n}\left\lceil\left(\Lambda_{1}, \Lambda_{2}\right)\right.$. This is because, by forgetfulness, we already have an appropriate parameter, namely: in 12.10, the structure $N=$ $N\left[\Lambda_{1}, \Lambda_{2}, M\right]$ is a model of $T_{\mathfrak{n}}^{0}$ where $\mathfrak{n}=\mathfrak{m} \uparrow_{\geq k}$.

Claim 12.12. Assume $\mathfrak{m}$ is forgetful. Assume $\left(\Lambda_{1}, \Lambda_{2}\right)$ are as in $12.9, N=$ $N\left[\Lambda_{1}, \Lambda_{2}, M\right]$ and $\mathfrak{n}=\mathfrak{m}\lceil\geq k$. Then:

(1) If $M \models T_{\mathfrak{m}}^{0}$, then $N \models T_{\mathfrak{n}}^{0}$.

(2) If $M \models T_{\mathfrak{m}}$, then:

(a) $N \models T_{\mathfrak{n}}$, and

(b) if $\bar{a}, \bar{b} \in{ }^{n} N$ and $\operatorname{tp}(\bar{a}, \emptyset, M)=\operatorname{tp}(\bar{b}, \emptyset, M)$, then $\operatorname{tp}(\bar{a}, \emptyset, N)=\operatorname{tp}(\bar{b}, \emptyset, N)$.

(3) If $M$ is a $\kappa$-saturated model of $T_{\mathfrak{m}}$, then $N$ is a $\kappa$-saturated model of $T_{\mathfrak{n}}$.

(4) If $M$ is a $\kappa$-special model of $T_{\mathfrak{m}}$, then $N$ is a $\kappa$-special model of $T_{\mathfrak{n}}$.

Proof. Easy, but as this is central we elaborate.

(1) There are two parts to check: the unary predicates match the structure of the tree, and the edges $R$ indeed reflect the instructions of $\mathcal{R}^{\mathfrak{n}}$. Since $\Lambda_{1} \times \Lambda_{2} \subseteq \mathcal{R}^{\mathfrak{m}}$, and $\mathfrak{m}$ is forgetful, $\mathfrak{n}=\mathfrak{m}\left\lceil\geq k\right.$ is well defined. Fix for a moment $\nu_{1} \in \mathcal{T}_{1, \ell}^{\mathfrak{n}}, \nu_{2} \in \mathcal{T}_{2, \ell}^{\mathfrak{n}}$. Then whenever $\left(\nu_{1}, \nu_{2}\right) \notin \mathcal{R}^{\mathfrak{n}}, T_{\mathfrak{n}}^{0}$ implies $\left(\forall x \in Q_{\nu_{1}}\right)\left(\forall y \in P_{\nu_{2}}\right)(\neg R(x, y))$. So let 
us check: in the model $N$,

$$
\begin{aligned}
& Q_{\nu_{1}}^{N}=\bigcup\left\{Q_{\eta^{`} \nu_{1}}^{M}: \eta \in \Lambda_{1}\right\} \\
& P_{\nu_{2}}^{N}=\bigcup\left\{P_{\eta^{-} \nu_{1}}^{M}: \eta \in \Lambda_{2}\right\} .
\end{aligned}
$$

Suppose $\left(\nu_{1}, \nu_{2}\right) \notin \mathcal{R}_{\mathfrak{n}, \ell}$. Then (as $\mathfrak{m}\lceil>k$ ) is well defined) for any choice of $(\eta, \rho) \in$ $\Lambda_{1} \times \Lambda_{2},\left(\eta^{\curlyvee} \nu_{1}, \rho^{\curlyvee} \nu_{2}\right) \notin \mathcal{R}_{\mathfrak{m}, k+\ell}$. Since for all $a \in Q_{\nu_{1}}^{N}$, and all $b \in P_{\nu_{2}}^{N}$, there are $\eta \in \Lambda_{1}$ and $\rho \in \Lambda_{2}$ such that $a \in Q_{\eta^{\urcorner} \nu_{1}}^{M}$ and $b \in P_{\rho\urcorner \nu_{2}}^{M}, M \models \neg R(a, b)$, so as $N$ changes no edges on its domain, also $N \models \neg R(a, b)$. So $\left(\forall x \in Q_{\nu_{1}}\right)(\forall y \in$ $\left.P_{\nu_{2}}\right)(\neg R(x, y))$ holds in $N$. Thus we verify the universal axioms of $T_{\mathfrak{n}}^{0}$.

(2), (3) are immediate from the fact that 12.9 gives an interpretation of $N$ in $M$.

One could prove (2) directly: since $T_{\mathfrak{n}}$ eliminates quantifiers, it suffices to check truth of relevant formulas as in the quantifier elimination argument 2.19.

For (3), we also sketch a direct proof as it will help for (4). Recall $M$ is $\kappa$ saturated. Convention: given a leaf, say $\rho \in \lim \left(\mathcal{T}_{2}^{n}\right)$, let us write $P_{\rho}^{M}$ to abbreviate $\bigcap\left\{P_{\rho\lceil n}^{M}: n<\omega\right\}$, and the parallel for $\lim \left(\mathcal{T}_{1}^{n}\right)$. Sometimes we will repeat this for emphasis. As $T_{\mathfrak{n}}$ has elimination of quantifiers, it suffices to verify that:

$(\star)_{1}$ if $\nu \in \lim \left(\mathcal{T}_{1}^{\mathfrak{n}}\right), X=\left\{\rho \in \lim \left(\mathcal{T}_{2}^{\mathfrak{n}}\right): \bigwedge_{m}\left(\nu\left\lceil m, \rho\lceil m) \in \mathcal{R}_{m}^{\mathfrak{n}}\right\}\right.\right.$, and $A, B \subseteq \bigcup\left\{P_{\rho}^{N}: \rho \in X\right\}$ are disjoint and $|A|+|B|<\kappa$, then for some $c \in Q_{\nu}^{N}=\bigcap_{n} Q_{\nu \uparrow n}^{N}$ we have that $(c, a) \in R^{N}$ for all $a \in A$, and $(c, b) \notin R^{N}$ for all $b \in B$.

as well as $(\star)_{2}$ the dual reversing the trees. In the case where $A, B$ are finite, this is the content of redoing the elimination of quantifiers as mentioned in (2).

As we are using $\Lambda_{1}, \Lambda_{2}$, symmetry holds and it suffices to prove $(\star)_{1}$. In particular it suffices to prove that for some $\eta \in \Lambda_{1}$ there is $c \in \bigcap\left\{Q_{\eta^{\wedge} \nu \uparrow n}^{M}: n<\omega\right\}$ such that $(c, a) \in R^{M}$ for all $a \in A$, and $(c, b) \notin R^{M}$ for all $b \in B$. Thus we move the problem to the model $M$.

In $M$, the sets $A$ and $B$ clearly remain disjoint. Let us locate the $a$ 's and $b$ 's. Since our $N$ was a model of $T_{\mathfrak{n}}$, for each $a \in A$, there is $\nu_{a} \in \lim \left(\mathcal{T}_{2}^{\mathfrak{n}}\right)$ such that $a \in P_{\nu_{a}}^{N}$, and likewise for each $b \in B$, there is $\nu_{b} \in \lim \left(\mathcal{T}_{2}^{\mathfrak{n}}\right)$ such that $b \in P_{\nu_{b}}^{N}$. Therefore in $M$, for each $a \in A$ there is $\rho_{a} \in \Lambda_{2}$ such that $a \in P_{\rho_{a} \frown \nu_{a}}^{M}$, and likewise for each $b \in B$ there is $\rho_{b} \in \Lambda_{2}$ such that $b \in P_{\rho_{b} \frown \nu_{b}}^{M}$, though the positive part is what is most important. Choose any $\eta \in \Lambda_{1}$. Since $\Lambda_{1} \times \Lambda_{2} \subseteq \mathcal{R}^{\mathfrak{m}}$, and by definition of $X$, we conclude that $\Lambda_{n}\left(\eta^{\wedge} \nu\left\lceil n, \rho_{a}{ }^{\frown} \nu_{a}\lceil n) \in \mathcal{R}_{n}^{\mathfrak{m}}\right.\right.$. So the type $p(x)$ saying that $\{R(x, a): a \in A\} \cup\{\neg R(x, b): b \in B\} \cup\left\{P_{\eta>\nu \mid n}(x): n<\omega\right\}$ is indeed a consistent partial type in $M$, and therefore there is such a $c \in|M|$ satisfying $(c, a) \in R^{M}$ for all $a \in A$, and $(c, b) \notin R^{M}$ for all $b \in B$, by saturation of $M$. Moreover, by definition of $p(x)$, it must be that $c \in P_{\eta}^{M}$, thus $c \in|N|$ by construction. This completes the proof.

(4) Similarly to (3).

Claim 12.13. Let $\mathcal{D}$ be a regular ultrafilter on $I,|I|=\lambda$. Suppose $\mathfrak{m}$ is a forgetful parameter (thus self-dual) and $k<\omega$. Suppose $\mathfrak{n}=\mathfrak{m}\lceil\geq k$. Then the following are equivalent:

(1) $\left(M_{\mathfrak{m}}\right)^{I} / \mathcal{D}$ is $\lambda^{+}$-saturated, for some, equivalently every, $M_{\mathfrak{m}} \models T_{\mathfrak{m}}$.

(2) $\left(M_{\mathfrak{n}}\right)^{I} / \mathcal{D}$ is $\lambda^{+}$-saturated, for some, equivalently every, $M_{\mathfrak{n}} \models T_{\mathfrak{n}}$. 
In other words, $T_{\mathfrak{m}}$ and $T_{\mathfrak{n}}$ are equivalent in Keisler's order 28

Proof. First note: for each given $\eta, \Lambda$, clearly we can interpret $N[\eta, \Lambda, M]$ in the model $M$. So as ultrapowers commute with reducts, $(N[\eta, \Lambda, M])^{I} / \mathcal{D}$ is canonically isomorphic to $N\left[\eta, \Lambda, M^{I} / \mathcal{D}\right]$. The parallel holds for $N[\Lambda, \eta, M]$, though since $\mathfrak{m}$ is forgetful therefore symmetric, it suffices to consider the first case.

(1) implies (2): As the ultrafilter is regular, it won't matter which model of $T_{\mathfrak{m}}$ or $T_{\mathfrak{n}}$ we choose (see Keisler [9] 2.1a), so we may as well choose $\eta \in \mathcal{T}_{1, k}^{\mathfrak{m}}, \rho \in \mathcal{T}_{2, k}^{\mathfrak{m}}$ and $M_{\mathfrak{n}}=N\left[\eta, \rho, M_{\mathfrak{m}}\right]$. Now apply 12.12 and the fact that ultrapowers commute with reducts.

(2) implies (1): Suppose (2). Then $\mathcal{D}$ is good for the theory of the random graph, because the theory of $M_{\mathfrak{n}}$ will be unstable and the theory of the random graph is minimum in Keisler's order among the unstable theories (see [16] §5).

Let $M_{\mathfrak{m}}^{*}=\left(M_{\mathfrak{m}}\right)^{I} / \mathcal{D}$. By Conclusion [5.6, it suffices to show that every partial type of $M_{\mathfrak{m}}^{*}$ of the form

$$
r(x)=\left\{Q_{\nu}(x)\right\} \cup\{R(x, a): a \in A\}
$$

is realized, where $\nu \in \mathcal{T}_{1}, A \subseteq M_{\mathfrak{m}}^{*}$ and $|A| \leq \lambda$. So $\nu$ has finite length, without loss of generality $\operatorname{lgn}(\nu) \geq k$, though of course $>k$ is allowed. Let $\eta=\nu\lceil k$.

Since $r$ is consistent, we may choose $\eta_{*}$ such that:

(a) $\eta^{\urcorner} \eta_{*} \in \lim \left(\mathcal{T}_{1}^{\mathfrak{m}}\right)$, and also $\nu \unlhd \eta^{\wedge} \eta_{*}$. [That is, we extend $\nu$ to a branch, but we write it as its restriction to $k$ plus the continuation.]

(b) and moreover the larger type

$$
r_{*}(x)=\left\{Q_{\eta^{\urcorner} \eta_{*} \mid \ell}(x): \ell<\omega\right\} \cup\{R(x, a): a \in A\}
$$

is still consistent.

Moreover, for each $a \in A$, we may choose $\nu_{a} \in \mathcal{T}_{2, k}$ and $\rho_{a}$ with $\nu_{a} \rho_{a} \in \lim \left(\mathcal{T}_{2}^{\mathfrak{n}}\right)$ such that for each $a \in A, M_{\mathfrak{m}}^{*} \models\left\{P_{\nu_{a}} \rho_{a} \mid \ell(a): \ell<\omega\right\}$. [Again we identify the branch of each $a \in A$, and write it as its restriction to $k$ plus the continuation.]

By the fact that $r_{*}$ is a [partial] type, note that

(c) if we write $\Lambda=\left\{\rho \in \mathcal{T}_{2, k}^{\mathfrak{m}}:(\eta, \rho) \in \mathcal{R}^{\mathfrak{m}}\right\}$, then $\nu_{a} \in \Lambda$ for each $a \in A$.

(d) moreover, for each $a \in A$ and each $\ell<\omega$,

$$
\left(\eta ^ { \frown } \eta _ { * } \left\lceil\ell, \nu_{a} \rho_{a}\lceil\ell) \in \mathcal{R}^{\mathfrak{m}} .\right.\right.
$$

Let $M_{\mathfrak{n}}=N\left[\eta, \Lambda, M_{\mathfrak{m}}\right]$. Then, recalling the beginning of the proof, we have that $M_{\mathfrak{n}}^{*}=N\left[\eta, \Lambda, M_{\mathfrak{m}}^{*}\right]=\left(N\left[\eta, \Lambda, M_{\mathfrak{m}}\right]\right)^{I} / \mathcal{D}$.

By (c), we have that $A \subseteq M_{\mathfrak{n}}^{*}$ [i.e. $\left.A \subseteq \operatorname{dom}\left(M_{\mathfrak{n}}^{*}\right)\right]$.

By (d) and the definition of forgetful, we have that $\left(\eta_{*}\left\lceil\ell, \rho_{a}\lceil\ell) \in \mathcal{R}^{\mathfrak{n}}\right.\right.$ for each $a \in A$ and $\ell<\omega$.

Thus $q_{*}(x)=\left\{Q_{\eta_{*} \ell \ell}(x): \ell<\omega\right\} \cup\{R(x, a): a \in A\}$ is a partial type in $M_{\mathfrak{n}}^{*}$. By our hypothesis (2), $M_{\mathfrak{n}}^{*}$ is $\lambda^{+}$-saturated, so $q_{*}$ is realized, say by $b$. By construction, $\operatorname{dom}\left(M_{\mathfrak{n}}^{*}\right) \subseteq \operatorname{dom}\left(M_{\mathfrak{m}}^{*}\right)$, so $b \in M_{\mathfrak{m}}^{*}$, indeed $M_{\mathfrak{m}}^{*} \models Q_{\eta}(b)$. Moreover, since $\nu$ (from the definition of $r(x)$ above) is an initial segment of $\eta^{\curvearrowleft} \eta_{*}, M_{\mathfrak{m}}^{*} \models Q_{\nu}(b)$. This shows that $b$ realizes $r(x)$ in $M_{\mathfrak{m}}^{*}$, which completes the proof.

The proofs just given show:

Conclusion 12.14. Let $\mathfrak{m}$ be a forgetful parameter.

\footnotetext{
${ }^{28}$ We pedantically avoid using the letter $N$ for a model in this proof to avoid confusion with the operation $N\left[\Lambda_{1}, \Lambda_{2}, M\right]$.
} 
(1) Suppose $M=T_{\mathfrak{m}} . M$ is $\lambda^{+}$-saturated if and only if for every pair $\left(\Lambda_{1}, \Lambda_{2}\right)$ such that $\Lambda_{1} \times \Lambda_{2} \subseteq \mathcal{R}^{\mathfrak{m}}$ and either $\left|\Lambda_{1}\right|=1$ or $\left|\Lambda_{2}\right|=1$, the model $N=N\left[\Lambda_{1}, \Lambda_{2}, M\right]$ is $\lambda^{+}$-saturated.

(2) Let $k<\omega$ and let $\mathfrak{n}=\mathfrak{m}\left\lceil\geq k\right.$. Then $T_{\mathfrak{m}}$, $T_{\mathfrak{n}}$ are equivalent in Keisler's order.

Recall that in the context of Convention 12.2 p. 60, our goal in this section is to compare theories of the form $T_{\mathfrak{m}[A]}$ and $T_{\mathfrak{m}[B]}$. The above shows that without loss of generality $A \subseteq B$, so Claim 12.15, which we now state, will follow from 12.24 below.

Claim 12.15. Suppose $A \subseteq^{*} B$. Then $T_{\mathfrak{m}[A]} \unlhd T_{\mathfrak{m}[B]}$.

Proof. This will follow from 12.24 below.

Recall that $\mathcal{Q}=Q_{\langle\rangle}, \mathcal{P}=P_{\langle\rangle}$. Write $\mathcal{T}_{\mathbf{i}}^{A}$ for $\mathcal{T}_{\mathbf{i}}^{\mathfrak{m}[A]}, \mathcal{T}_{\mathbf{i}}^{B}$ for $\mathcal{T}_{\mathbf{i}}^{\mathfrak{m}[B]}$ and $\mathbf{i}=1,2$, and $\mathcal{R}^{A}=\mathcal{R}^{\mathfrak{m}[A]}, \mathcal{R}^{B}=\mathcal{R}^{\mathfrak{m}[B]}$.

Observation 12.16. As $A \subseteq B$, we have that:

- $\mathcal{T}_{\mathbf{i}}^{A}=\mathcal{T}_{\mathbf{i}}^{B}$ for $\mathbf{i}=1,2$.

- $\mathcal{R}^{B} \subseteq \mathcal{R}^{A}$.

\section{Convention 12.17. Notation:}

(1) If $a \in \mathcal{P}^{M}$, saying that " $\rho$ is the leaf of a" means that $\rho$ is the unique element of $\lim \left(\mathcal{T}_{2}^{M}\right)$ such that $M \models P_{\rho \uparrow \ell}(a)$ for $\ell<\omega$, and similarly for $a \in \mathcal{Q}^{M}$ and $\eta \in \lim \left(\mathcal{T}_{1}^{M}\right)$.

(2) Write $(\eta, \rho) \in \mathcal{R}_{\infty}^{A}$ to mean that $\eta \in \lim \left(\mathcal{T}_{1}^{A}\right), \rho \in \lim \left(\mathcal{T}_{2}^{A}\right)$ and for all $\ell<\omega,\left(\eta\left\lceil\ell, \rho\lceil\ell) \in \mathcal{R}^{A}\right.\right.$.

Observation 12.18. Suppose $n<\omega, Y \subseteq \mathcal{P}^{M_{A}}$ is finite, $\eta \in \mathcal{T}_{1}^{A}$. For $a \in Y$ let $\rho_{a} \in \lim \left(\mathcal{T}_{2}^{A}\right)$ be the leaf of a. Let $M_{A} \models T_{\mathfrak{m}[A]}$. The following are equivalent:

(1) $M_{A} \models(\exists x) \bigwedge_{a \in Y}\left(Q_{\eta}(x) \wedge R(x, a)\right)$

(2) there is $\nu \in \lim \left(\mathcal{T}_{1}^{A}\right), \eta \unlhd \nu$ such that $\left(\nu, \rho_{a}\right) \in \mathcal{R}_{\infty}^{A}$ for each $a \in Y$.

Observation 12.19 (Discussion/Observation). Suppose we are in a model $M_{A} \models$ $T_{A}, \eta \in \mathcal{T}_{1}^{A}[\operatorname{so} \operatorname{lgn}(\eta)<\omega]$, and we have a finite set of formulas [not necessarily a partial type] of the form

$$
\left\{Q_{\eta}(x)\right\} \cup\{R(x, a): a \in X\}
$$

which we may write for uniformity as a sequence, possibly with repetitions:

$$
p(x)=\left\langle Q_{\eta}(x) \wedge R(x, a): a \in X\right\rangle .
$$

For each $a \in X$, let $\rho_{a} \in \lim \left(\mathcal{T}_{2}^{A}\right)$ be the leaf of $a$. Then the "pattern" of this set of formulas in $M_{A}$ is captured by the data of: for which $\sigma \subseteq X$ is it the case that there exists $\eta_{*} \in \lim \left(\mathcal{T}_{1}^{A}\right)$ such that (i) $\eta \unlhd \eta_{*}$ and (ii) $\left(\eta_{*}, \rho_{a}\right) \in \mathcal{R}_{\infty}^{A}$ for all $a \in X$.

Discussion 12.20. One obstruction to the natural try at $A \subseteq B$ implies $T_{A} \unlhd T_{B}$ is: Consider $M_{A} \models T_{A}, M_{B} \models T_{B}, \mathcal{D}$ a regular ultrafilter on $I, M_{A}^{*}=\left(M_{A}\right)^{I} / \mathcal{D}$, $M_{B}^{*}=\left(M_{B}\right)^{I} / \mathcal{D}$. Now we might like to show that given a certain type of $M_{A}^{*}$, we can copy it to a type of $M_{B}^{*}$ and apply saturation of the second to solve the first. A natural way to copy is to assign finitely many formulas of $p$ to each index $t \in I$, using the regularizing family, and then copy this finite pattern from $M_{A}$ to $M_{B}$. The 
natural way to copy patterns is to use the same leaves, but even though $\mathcal{T}_{\mathbf{i}}{ }^{A}=\mathcal{T}_{\mathbf{i}}{ }^{B}$, the same leaves may give a different pattern in $M_{B}$ as $\mathcal{R}^{A} \supsetneq \mathcal{R}^{B}$.

Instead, we show that given $M_{A}$ and a finite pattern there, we can first modify the leaves involved a little without changing the pattern (informally, we make sure there is no 'spreading out' on $k \in \omega \backslash A$ ), and second, that any such modified set of leaves behaves as desired in $\mathcal{R}^{B}$. This allows the plan to go through.

Claim 12.21. Recalling that $\mathfrak{m}[A]$ and $\mathfrak{m}[B]$ are forgetful, suppose we are given $\left\langle Q_{\eta}(x) \wedge R(x, a): a \in X\right\rangle$ in $M_{A}$ where:

- $\eta \in \mathcal{T}_{1}^{A}$

- $X$ finite,

- for each $a \in X, M_{A} \models(\exists x)\left(Q_{\eta}(x) \wedge R(x, a)\right)$

- thus we are given $\left\langle\rho_{a}: a \in X\right\rangle$ where $\rho_{a} \in \lim \left(\mathcal{T}_{2}^{A}\right)$ is the leaf of a.

Let $\left\langle\rho_{a}^{*}: a \in X\right\rangle \in{ }^{|X|} \lim \left(\mathcal{T}_{2}^{B}\right)$ satisfy the following two conditions:

(a) for $k \in A$ and $a \in X, \rho_{a}^{*}(k)=\rho_{a}(k)$

(b) for $k \in \omega \backslash A$, for all $a \in X, \rho_{a}^{*}(k)=0$

Then for some $\nu \in \mathcal{T}_{1}^{B}$ with $\operatorname{lgn}(\nu)=\operatorname{lgn}(\eta)$, for every $\sigma \subseteq X$, the following are equivalent:

(1) there exists $\eta_{\sigma} \in \lim \left(\mathcal{T}_{1}^{A}\right), \eta \unlhd \eta_{\sigma}$ and $\left(\eta_{\sigma}, \rho_{a}\right) \in \mathcal{R}_{\infty}^{A}$ for every $a \in \sigma$

(2) there exists $\nu_{\sigma} \in \lim \left(\mathcal{T}_{1}^{B}\right), \nu \unlhd \nu_{\sigma}$ and $\left(\nu_{\sigma}, \rho_{a}^{*}\right) \in \mathcal{R}_{\infty}^{B}$ for every $a \in \sigma$.

Informally, "the pattern remains unchanged and transfers to $M_{B}$ ".

Remark 12.22. Continuing in the notation of Claim [12.21, it will follow from that Claim and from Observation 12.18 that if we choose distinct elements $b_{a} \in\left|M_{B}\right|$ for $a \in X$ so that for each $a \in X, \rho_{a}^{*}$ is the leaf of $b_{a}$ in $M_{B}$, then for any $\sigma \subseteq X$, the following are equivalent:

$(\star)_{1} M_{A} \models(\exists x) \bigwedge_{a \in \sigma}\left(Q_{\eta}(x) \wedge R(x, a)\right)$.

$(\star)_{2} \quad M_{B} \models(\exists x) \bigwedge_{a \in \sigma}\left(Q_{\nu}(x) \wedge R\left(x, b_{a}\right)\right)$.

Proof of Claim 12.21. Note: the construction in the proof will give a $\nu$ which depends only on $\eta$. In particular: for each $k<\operatorname{lgn}(\eta)$, define $\nu(k)$ by:

- if $k \in A, \nu(k)=\eta(k)$

- if $k \in \omega \backslash A, \nu(k)=\min \left\{i<m_{k}:(i, 0) \in E^{k}\right\}$ which exists by the definition of "small" in the construction of the graphs $\bar{E}$.

Note that if $\operatorname{lgn}(\eta) \subseteq A$, then this gives $\nu=\eta$.

(1) implies (2). By (1) there is such an $\eta_{\sigma}$. Define $\nu_{\sigma} \in \lim \left(\mathcal{T}_{1}^{B}\right)$ [recalling $\left.\mathcal{T}_{1}^{B}=\mathcal{T}_{1}^{A}\right]$ as follows: for each $k<\omega$, define $\nu_{\sigma}(k)$ by:

(i) if $k \in A, \nu_{\sigma}(k)=\eta_{\sigma}(k)$

(ii) if $k \in \omega \backslash A, \nu_{\sigma}(k)=\min \left\{i<m_{k}:(i, 0) \in E^{k}\right\}$ which exists by the definition of "small" in the construction of the graphs $\bar{E}$.

Then recall $\eta \unlhd \eta_{\sigma}$ hence $\nu \unlhd \nu_{\sigma}$. For each $a \in \sigma$, we will prove by induction on $\ell<\omega$ that

$$
(\star)_{\ell} \quad\left(\eta _ { \sigma } \left\lceil\ell, \rho_{a}\lceil\ell) \in \mathcal{R}^{A} \Longrightarrow\left(\nu _ { \sigma } \left\lceil\ell, \rho_{a}^{*}\lceil\ell) \in \mathcal{R}^{B} .\right.\right.\right.\right.
$$

For $\ell=0$ this is trivially true as $(\langle\rangle,\langle\rangle) \in \mathcal{R}^{A} \cap \mathcal{R}^{B}$. Suppose $(\star)_{\ell}$ holds. If $\ell \in A$, then $\nu_{\sigma}(\ell)=\eta_{\sigma}(\ell)$ and $\rho_{a}(\ell)=\rho_{a}^{*}(\ell)$, so by inductive hypothesis and forgetfulness, $(\star)_{\ell+1}$ is immediate. If $\ell \notin A$, then by inductive hypothesis and $(i i)$,

$$
\left(\left(\nu_{\sigma}\lceil\ell)^{\frown}\left\langle\nu_{\sigma}(\ell)\right\rangle,\left(\rho_{a}^{*}\lceil\ell)^{\frown}\langle 0\rangle\right) \in \mathcal{R}^{B}\right.\right.
$$


i.e. $\left(\nu_{\sigma}\left\lceil\ell+1, \rho_{a}^{*}\lceil\ell+1) \in \mathcal{R}^{B}\right.\right.$, so $(\star)_{\ell+1}$ holds.

This proves $(\star)_{\ell+1}$ and so proves this direction.

(2) implies (1). Suppose there is such a $\nu_{\sigma}$. Write $\nu_{\sigma}=\nu^{\wedge} \nu_{\infty}$. Let $\eta_{\sigma}=\eta^{\wedge} \nu_{\infty}$. So clearly $\eta \unlhd \eta_{\sigma}$. For each $a \in \sigma$, we will prove by induction on $\ell<\omega$ that

$$
\oplus_{\ell} \quad\left(\nu _ { \sigma } \left\lceil\ell, \rho_{a}^{*}\lceil\ell) \in \mathcal{R}^{B} \Longrightarrow\left(\eta _ { \sigma } \left\lceil\ell, \rho_{a}\lceil\ell) \in \mathcal{R}^{A} .\right.\right.\right.\right.
$$

For $\ell \leq \operatorname{lgn}(\eta)$ this is trivially true as it follows from the assumptions of the Claim (third bullet point) that $\left(\eta, \rho_{a}\lceil\operatorname{lgn}(\eta)) \in \mathcal{R}^{A}\right.$ for each $a \in X$, so also for each $a \in \sigma$. Suppose $\oplus_{\ell}$ holds and $\ell \geq \operatorname{lgn}(\eta)$. If $\ell \in A$, then $\eta_{\sigma}(\ell)=\nu_{\sigma}(\ell)$ and $\rho_{a}(\ell)=\rho_{a}^{*}(\ell)$, so $\oplus_{\ell+1}$ follows by inductive hypothesis and forgetfulness. If $\ell \in \omega \backslash A$, then $\ell$ is a lazy level for $A$, so $\oplus_{\ell+1}$ is immediate from $\oplus_{\ell}$. This proves $\oplus_{\ell+1}$, which finishes this direction and so finishes the proof.

Lemma 12.23. Let $\mathcal{D}$ be a regular ultrafilter on $I,|I|=\lambda$. Let $M_{A}^{*}=\left(M_{A}\right)^{I} / \mathcal{D}$. Let $M_{B}^{*}=\left(M_{B}\right)^{I} / \mathcal{D}$. Suppose $p(x)=\left\{Q_{\eta}(x)\right\} \cup\{R(x, a): a \in X\}$ is a partial type of $M_{A}$, with $\eta \in \mathcal{T}_{1}^{A}$ and $|X| \leq \lambda$. There is a partial type $q(x)=\left\{Q_{\eta}(x)\right\} \cup$ $\left\{R\left(x, b_{a}\right): a \in X\right\}$ of $M_{B}^{*}$, such that if $q(x)$ is realized in $M_{B}^{*}$, then $p(x)$ is realized in $M_{A}^{*}$.

Proof. Let $\left\{X_{a}: a \in X\right\} \subseteq \mathcal{D}$ be a regularizing family for $\mathcal{D}$. Enumerate $p$ as $\left\langle Q_{\eta}(x) \wedge R(x, a): a \in X\right\rangle$, so here $X$ may be infinite. Let $f:[X]^{<\aleph_{0}} \rightarrow \mathcal{D}$ be the map given by

$$
u \mapsto\left\{t \in I: M_{A} \models(\exists x) \bigwedge_{a \in u}\left(Q_{\eta}(x) \wedge R(x, a)\right)\right\} \cap \bigcap_{a \in u} X_{a} .
$$

In particular, for each $t \in I, X_{t}=\{a \in X: t \in f(\{a\})\}$ is finite.

We define the parameters for $q$ (what will be the corresponding type in $M_{B}^{*}$ ) coordinatewise. For each $t \in I$, apply Claim 12.21 to the following sequence of formulas of $M_{A}$ :

$$
p_{t}(x)=\left\langle Q_{\eta}(x) \wedge R(x, a[t]): a \in X_{t}\right\rangle .
$$

Let $\nu_{t}$ and $\left\langle\rho_{t, a}^{*}: a \in X_{t}\right\rangle$ be as returned by that Claim. Observe that we have $\nu_{t}$ defined for every $t \in I$, but $\left\{\nu_{t}: t \in I\right\}$ is finite, so for some $J \in \mathcal{D},\left\langle\nu_{t}: t \in J\right\rangle$ is constant. [Alternately, by the first sentence of the proof of Claim 12.21, $\nu_{t}$ depends only on $\eta$, so in fact we obtain the same $\nu_{t}$ for every $t \in I$.] So we call it $\nu$.

For $a \in X_{t}$, choose $b_{t, a}$ to be pairwise distinct elements of $P_{\rho_{t, a}^{*}}^{M_{B}}$. For $a \in X \backslash X_{t}$, choose $b_{t, a}$ to be any element of $P_{\emptyset}^{M_{B}}$. Recalling 12.22, we have that for any $\sigma \subseteq X$, the following are equivalent:

(1) $M_{A} \models(\exists x) \bigwedge_{a \in \sigma}\left(Q_{\eta}(x) \wedge R(x, a[t])\right)$

(2) $M_{B} \models(\exists x) \bigwedge_{a \in \sigma}\left(Q_{\nu}(x) \wedge R\left(x, b_{t, a}\right)\right)$

For each $a \in X$, define $b_{a}=\left\langle b_{t, a}: t \in I\right\rangle / \mathcal{D}$, so $b_{a} \in M_{B}^{*}$. Consider

$$
q(x)=\left\langle Q_{\eta}(x) \wedge R\left(x, b_{a}\right): a \in X\right\rangle .
$$

Then, since $q$ has the same 'pattern' as $p$ at each index $t \in I$ under the function $f$, and $p$ is a partial type in $M_{A}^{*}$, we have that $q(x)$ is also a partial type of $M_{B}^{*}$, and moreover, if $q$ is realized in $M_{B}^{*}$ then also $p$ is realized in $M_{A}^{*}$.

Corollary 12.24. If $A \subseteq B$, then $T_{A} \unlhd T_{B}$. 
We include a slightly more general result for $\unlhd^{*}$, the interpretability order. For the reader interested in Keisler's order, this is not essential to our main arguments.

Lemma 12.25. Suppose $\mathfrak{m}_{1}, \mathfrak{m}_{2}$ are forgetful and $\mathfrak{m}_{1}\left\lceil_{\geq k}=\mathfrak{n}=\mathfrak{m}_{2}\left\lceil\geq k\right.\right.$. Then $T_{\mathfrak{m}_{1}}$, $T_{\mathfrak{n}}, T_{\mathfrak{m}_{2}}$ are $\unlhd^{*}$-equivalent, thus $\unlhd$-equivalent.

Proof. Let $M_{1}, M, M_{2}$ be models of $T_{\mathfrak{m}_{1}}, T_{\mathfrak{n}}, T_{\mathfrak{m}_{2}}$ respectively such that all three are special of cardinality $\mu=\beth_{\delta}, \delta$ limit. Let $\chi$ be such that $M_{1}, M, M_{2} \in \mathcal{H}(\chi)$. Let $\mathfrak{B}_{*}$ be the model $\left(\mathcal{H}(\chi), \in, M_{1}, M, M_{2}\right)$ or an expansion of it. Note first that for $\Lambda_{1} \times \Lambda_{2} \subseteq \mathcal{R}_{k}^{\mathfrak{m}_{1}}, M$ is isomorphic to $N\left[\Lambda_{1}, \Lambda_{2}, M_{1}\right]$. [Why? $M_{1}$ is a special model of $T_{\mathfrak{m}_{1}}$, so by Claim 12.12 (4), so is $N\left[\Lambda_{1}, \Lambda_{2}, M_{1}\right] \models T_{\mathfrak{n}}$. By assumption $M \models T_{\mathfrak{n}}$ is special. So by the uniqueness of special models, they must be isomorphic.] The parallel holds replacing $M_{1}$ by $M_{2}$. These isomorphisms are recorded by $\mathfrak{B}_{*}$. Hence they remain in any $\mathfrak{B} \equiv \mathfrak{B}_{*}$. That is,

$$
\mathfrak{B}_{*} \models M \cong N\left[\Lambda_{1}, \Lambda_{2}, M_{\mathbf{i}}\right]
$$

for $\mathbf{i}=1,2$, and $\Lambda_{1}, \Lambda_{2}$ as above. Moreover, for any $\mathfrak{B} \equiv \mathfrak{B}_{*}$,

$$
\mathfrak{B} \models " M \cong N\left[\Lambda_{1}, \Lambda_{2}, M_{\mathbf{i}}\right] "
$$

(as $M, M_{1}, M_{2}$ are definable elements). Note also that

$$
\left(N\left[\Lambda_{1}, \Lambda_{2}, M\right]\right)^{\mathfrak{B}}=N\left[\Lambda_{1}, \Lambda_{2}, M_{\mathbf{i}}^{\mathfrak{B}}\right], \mathbf{i}=1,2
$$

by absoluteness. (Pedantically, if $\mathfrak{B}$ is not well-founded, the set $\left\{P_{\eta}: \mathfrak{B} \models P_{\eta} \in\right.$ $\left.\tau\left(M_{\mathbf{i}}\right)\right\}$ may have non-standard elements, but no harm.) Thus, for $\mathbf{i}=1,2$,

$N\left[\Lambda_{1}, \Lambda_{2}, M_{\mathfrak{i}}^{\mathfrak{B}}\right]$ is a special model of $T_{\mathfrak{n}}$ of cardinality $\mu$ isomorphic to $M^{\mathfrak{B}}$ and so by transitivity, as both are isomorphic to $M^{\mathfrak{B}}$,

$$
N\left[\Lambda_{1}, \Lambda_{2}, M_{1}^{\mathfrak{B}}\right] \cong N\left[\Lambda_{1}, \Lambda_{2}, M_{2}^{\mathfrak{B}}\right] .
$$

So $T h\left(\mathfrak{B}_{*}\right)$ witnesses that $T_{\mathfrak{m}_{1}}, T_{\mathfrak{n}}, T_{\mathfrak{m}_{2}}$ are $\unlhd^{*}$-equivalent. Why? $M_{\mathfrak{i}}^{\mathfrak{B}}$ is $\kappa$-saturated if and only if all the models of the form $N\left[\Lambda_{1}, \Lambda_{2}, M_{\mathrm{i}}^{\mathfrak{B}}\right]$ for $\Lambda_{1} \times \Lambda_{2} \subseteq \mathcal{R}_{k}^{\mathfrak{m}_{\mathbf{i}}}$ are $\kappa$ saturated, if and only if $M^{\mathfrak{B}}$ is $\kappa$-saturated, which suffices.

Claim 12.26. Suppose $B \backslash A$ is infinite. Then $\neg\left(T_{\mathfrak{m}[B]} \unlhd T_{\mathfrak{m}[A]}\right)$.

Proof. Just as in the main argument above: as long as $B \backslash A$ is infinite, we have enough "independence" between the parameters for this direction. That is, consider the chain condition Definition 8.2 with the cosmetic difference that we write $A, B$ instead of using level functions: really, we could define $\xi_{\mathfrak{m}[A]}$ to be 1 if $n \in A$ and 0 otherwise, and $\xi_{\mathfrak{m}[B]}$ to be 1 if $n \in B$ and 0 otherwise. Then if $\mathcal{I}$ is the ideal generated by $\{A\} \cup[\omega]^{<\aleph_{0}}$, clearly $B \neq \emptyset \bmod \mathcal{I}$ so we may quote 9.4, Thus, it remains possible to construct an ultrafilter which is good for $T_{\mathfrak{m}[A]}$ while preserving the fact that it is not good for $T_{\mathfrak{m}[B]}$.

So we arrive at:

Theorem 12.27. We can find $T_{A}$ for $A \subseteq \omega$ such that $T_{A} \unlhd T_{B}$ if and only if $A \subseteq B$ mod finite.

Proof. We use the family $\left\{T_{\mathfrak{m}[A]}: A \subseteq \omega\right\}$ defined above. If $A \subseteq B \bmod$ finite, apply 12.15. On the other hand, if $B \backslash A$ is infinite, apply Lemma 12.26,

Thus for $A, B \subseteq \omega$,

$$
T_{\mathfrak{m}[A]} \unlhd T_{\mathfrak{m}[B]} \text { if and only if } A \subseteq^{*} B .
$$


This is what we hoped to prove.

In fact we have shown more:

Conclusion 12.28. Suppose we consider the family $\{\mathfrak{m}[A]: A \subseteq \omega\}$ of parameters defined at the beginning of this section. Then:

(1) $A \subseteq{ }^{*} B \Longrightarrow T_{\mathfrak{m}[A]} \unlhd T_{\mathfrak{m}[B]}$, indeed $T_{\mathfrak{m}[A]} \unlhd^{*} T_{\mathfrak{m}[B]}$

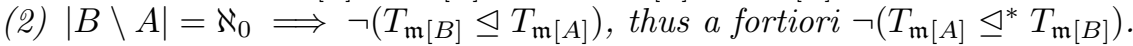

This completes the proof of Theorem 11.9

To motivate the second theorem of this section, remember that when we were partitioning $\mathbf{M}_{*}$ into $\mathcal{M}$ and $\mathcal{N}$ in the previous section, we were essentially choosing a partition of independent subsets of $\omega$. We may ask about which partitions are possible of all families of subsets of $\omega$. The next theorem answers this question: it is 11.10 above, which we restate for convenience.

Theorem (Theorem 11.10). There is a family of parameters $\{\mathfrak{m}[A]: A \subseteq \omega\}$ such that each $T_{\mathfrak{m}[A]}$ is countable, complete, simple, and low, and the following are equivalent for any $\lambda \geq 2^{\aleph_{0}}$ and any set $\mathcal{X} \subseteq \mathcal{P}(\omega)$ :

(1) There exists a regular ultrafilter $\mathcal{D}$ on $\lambda$ such that $\mathcal{X}=\{A \subseteq \omega: \mathcal{D}$ is $\left(\lambda^{+}, T_{\mathfrak{m}[A]}\right)$-good $\}$.

(2) $\mathcal{X} \supseteq[\omega]^{<\aleph_{0}}$ is an ideal.

Proof of Theorem 11.10, Fix $\mathcal{X} \subseteq \mathcal{P}(\omega)$, and fix $\lambda \geq 2^{\aleph_{0}}$.

$(2) \rightarrow(1)$ : Immediate from the earlier construction: simply choose the ideal $\mathcal{I}$ in the chain condition 8.2 to be our $\mathcal{X}$.

$(1) \rightarrow(2)$ : If $A \in \mathcal{X}$ and $B \subseteq \subseteq^{*} A$, then $\mathcal{D}$ is $\left(\lambda^{+}, T_{\mathfrak{m}[B]}\right)$-good by 12.15]above. So to show that $\mathcal{X}$ is an ideal which extends the finite sets, it suffices to show that if $A \in \mathcal{X}$ and $B \in \mathcal{X}$, then $A \cup B$ is in $\mathcal{X}$. In other words, we shall fix $A, B \subseteq \omega$, and assume that $\mathcal{D}$ is a regular ultrafilter on $|I|,|I|=\lambda \geq 2^{\aleph_{0}}$ which is $\left(\lambda^{+}, T_{\mathfrak{m}[A]}\right)$-good and $\left(\lambda^{+}, T_{\mathfrak{m}[B]}\right)$-good, and we shall show that $\mathcal{D}$ is $\left(\lambda^{+}, T_{\mathfrak{m}[A \cup B]}\right)$-good 29

Since $T_{\mathfrak{m}[A]}$ and $T_{\mathfrak{m}[B]}$ are both simple unstable, it follows that $\mathcal{D}$ is good for the theory of the random graph. Choose $M_{A}, M_{B}, M_{A \cup B}$ to be $\aleph_{1}$-saturated models of $T_{\mathfrak{m}[A]}, T_{\mathfrak{m}[B]}$, and $T_{\mathfrak{m}[A \cup B]}$ respectively. Let $M_{A}^{*}, M_{B}^{*}, M_{A \cup B}^{*}$ be the respective ultrapowers using $\mathcal{D}$. As usual, to show that $M_{A \cup B}^{*}$ is $\lambda^{+}$-saturated, it suffices to prove that all partial types of the form

$$
r(x)=\left\{Q_{\eta}(x) \cup\{R(x, c): c \in C\}\right.
$$

are realized, where $\eta \in \mathcal{T}_{1}^{\mathfrak{m}[A \cup B]}$ and $|C| \leq \lambda$. Fix such an $r$. Without loss of generality, we will assume $|C|=\lambda$.

Let $\left\{Y_{c}: c \in C\right\} \subseteq \mathcal{D}$ be a regularizing family for $\mathcal{D}$. 30 Let

$$
d:[C]^{<\aleph_{0}} \rightarrow \mathcal{D}
$$

\footnotetext{
${ }^{29}$ Informally, if $\mathcal{D}$ can handle types coming from trees where the levels in $A$ are active, and trees where the levels in $B$ are active, then it can handle types coming from trees where the levels in $A \cup B$ are active.

${ }^{30}$ i.e. any element of the family belongs to $\mathcal{D}$, but the intersection of any infinitely many elements of this family is empty - exists by definition of regular ultrafilter.
} 
be the map given by:

$$
u \mapsto\left\{t \in I: M_{A \cup B} \models \exists x \bigwedge_{c \in u}\left(Q_{\eta}(x) \wedge R(x, c[t])\right)\right\} \cap \bigcap_{c \in u} Y_{c} .
$$

Note that $d$ is monotonic $(u \subseteq v$ implies $d(u) \supseteq d(v))$, and for each $t \in I$, the set $C_{t}=\{c \in C: t \in d(\{c\})\}$ is finite.

For each $t$ and $c \in C$, there is a leaf $\rho_{c, t} \in \lim \left(\mathcal{T}_{2}^{\mathfrak{m}[A]}\right)=\lim \left(\mathcal{T}_{2}^{\mathfrak{m}[B]}\right)$ such that $M \models P_{\rho_{c, t} \ell \ell}(c[t])$ for all $\ell<\omega$. For each $t \in I$ and each $c \in C$ choose $31 a_{c, t}$ to be any element of $M_{A}$ such that $M_{A} \models P_{\rho_{c, t} \mid \ell}\left(a_{c, t}\right)$ for all $\ell<\omega$ and choose

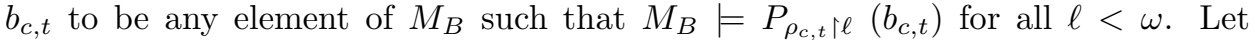
$a_{c}=\left\langle a_{c, t}: t \in I\right\rangle / \mathcal{D} \in M_{A}^{*}$, and let $b_{c}=\left\langle b_{c, t}: t \in I\right\rangle / \mathcal{D} \in M^{*} / B$. [Without loss of generality, $a_{c}[t]=a_{c, t}$ and $\left.b_{c}[t]=b_{c, t}.\right]$ Consider

$$
r_{A}=\left\{Q_{\eta}(x)\right\} \cup\left\{R\left(x, a_{c}\right): c \in C\right\}
$$

and consider

$$
r_{B}=\left\{Q_{\eta}(x)\right\} \cup\left\{R\left(x, b_{c}\right): c \in C\right\} .
$$

Observe that $r_{A}(x)$ is a partial type in $M_{A}^{*}$ since $\mathcal{R}^{\mathfrak{m}[A]} \supseteq \mathcal{R}^{\mathfrak{m}[A \cup B]}$, and likewise $r_{B}(x)$ is a partial type in $M_{B}^{*}$ since $\mathcal{R}^{\mathfrak{m}[B]} \supseteq \mathcal{R}^{\mathfrak{m}[A \cup B]}$. By our assumption on $\mathcal{D}$, $r_{A}$ is realized, say by $a_{*} \in M_{A}^{*}$, and $r_{B}$ is also realized, say by $b_{*} \in M_{B}^{*}$. Let us define $d_{*}:[C]^{<\aleph_{0}} \rightarrow \mathcal{D}$ to be the refinement of $d$ given by:

$$
d_{*}(u)=d(u) \cap\left\{t \in I: M_{A} \models R\left(a_{*}[t], a_{c, t}\right)\right\} \cap\left\{t \in I: M_{B} \models R\left(b_{*}[t], b_{c, t}\right)\right\} .
$$

Thus, for each $t \in I$, we may define $C_{t}^{*}=\left\{c \in C: t \in d_{*}(\{c\})\right\}$. It follows from the definition of $d_{*}$ that for each $t \in I, C_{t}^{*} \subseteq C_{t}$, thus $C_{t}^{*}$ is finite; moreover, for each $t \in I$,

$$
M_{A} \models(\exists x)\left(Q_{\eta}(x) \wedge \bigwedge_{c \in C_{t}^{*}} R\left(x, a_{c, t}\right)\right)
$$

[in particular $a_{*}[t]$ is such a witness] and likewise

$$
M_{B} \models(\exists x)\left(Q_{\eta}(x) \wedge \bigwedge_{c \in C_{t}^{*}} R\left(x, b_{c, t}\right)\right)
$$

[in particular $b_{*}[t]$ is such a witness]. Fix $t \in I$. We now aim to prove:

$$
\left.M_{A \cup B} \models(\exists x)\left(Q_{\eta}(x) \wedge \bigwedge_{c \in C_{t}^{*}} R(x, c[t])\right)\right) .
$$

Recall that

(i) $\mathcal{T}_{1}^{\mathfrak{m}[A \cup B]}=\mathcal{T}_{1}^{\mathfrak{m}[A]}=\mathcal{T}_{1}^{\mathfrak{m}[B]}$

(ii) $\mathcal{R}^{\mathfrak{m}[A \cup B]}$ is contained in each of $\mathcal{R}^{\mathfrak{m}[A]}$ and $\mathcal{R}^{\mathfrak{m}[B]}$.

(iii) for each $\ell<\omega$, for some, equivalently every, choice o 32

$(\eta, \rho) \in \mathcal{R}_{\ell}^{\mathfrak{m}[A \cup B]},\left(\eta_{A}, \rho_{A}\right) \in \mathcal{R}_{\ell}^{\mathfrak{m}[A]},\left(\eta_{B}, \rho_{B}\right) \in \mathcal{R}_{\ell}^{\mathfrak{m}[B]}$

we have that: if $\ell \in A$, then for all $i, j<m_{\ell}$,

$$
\left(\eta^{\curlyvee}\langle i\rangle, \rho^{\curlyvee}\langle j\rangle\right) \in \mathcal{R}^{\mathfrak{m}[A \cup B]} \text { if and only if }\left(\eta_{A} \curlyvee\langle i\rangle, \rho_{A} \curlyvee\langle j\rangle\right) \in \mathcal{R}^{\mathfrak{m}[A]}
$$

\footnotetext{
${ }^{31}$ One may follow these instructions for $t \in I$ and $c \in C_{t}$, and otherwise choose arbitrarily.

${ }^{32}$ Note there is always some such choice, by the extension axioms.
} 
and if $\ell \in B$, then for all $i, j<m_{\ell}$,

$$
\left(\eta^{\Upsilon}\langle i\rangle, \rho^{\curlyvee}\langle j\rangle\right) \in \mathcal{R}^{\mathfrak{m}[A \cup B]} \text { if and only if }\left(\eta_{B}\left\langle\langle i\rangle, \rho_{B} \curlyvee\langle j\rangle\right) \in \mathcal{R}^{\mathfrak{m}[B]}\right.
$$

and otherwise, if $\ell \in \omega \backslash A \cup B$, for all $i, j<m_{\ell}$,

$$
\left(\eta^{\frown}\langle i\rangle, \rho^{\frown}\langle j\rangle\right) \in \mathcal{R}^{\mathfrak{m}[A \cup B]} .
$$

[Of course if $\ell \in A \cap B$ then the two relevant conditions hold simultaneously.]

Recall that for each $c \in C_{t}^{*}$ we had defined its leaf $\rho_{c, t}$. It would suffice to prove that there is $\eta_{*} \in \lim \left(\mathcal{T}_{1}^{\mathfrak{m}[A \cup B]}\right)$ so that

$$
\left(\eta _ { * } \left\lceils, \rho_{c, t}\lceil s) \in \mathcal{R}^{\mathfrak{m}[A \cup B]} \text { for all } c \in C_{t}^{*} \text { and all } s<\omega .\right.\right.
$$

Let $\eta_{a}$ be the leaf of $a_{*}$, i.e. the unique element of $\lim \left(\mathcal{T}_{1}^{\mathfrak{m}[A]}\right)$ such that $M_{A} \models$ $Q_{\eta_{a} \nmid \ell}\left(a_{*}[t]\right)$ for all $\ell<\omega$, and let $\eta_{b}$ be the leaf of $b_{*}[t]$, i.e. the unique element of $\lim \left(\mathcal{T}_{1}^{\mathfrak{m}[B]}\right)$ such that $M_{B} \models Q_{\eta_{b} \mid \ell}\left(b_{*}[t]\right)$ for all $\ell<\omega$. So necessarily $\eta \unlhd \eta_{a}$ and $\eta \unlhd \eta_{b}$. Suppose first that $\eta_{a}=\eta_{b}$. Let $\eta_{*} \in \lim \left(\mathcal{T}_{1}^{\mathfrak{m}[A \cup B]}\right)$ be given by $\eta_{*}=\eta_{a}=\eta_{b}$. This $\eta_{*}$ satisfies (dd), as is easy to verify by inductively applying (iii) above.

If not, suppose that there is some $\mathbf{i}<\omega$ minimal for the property that 33 we have $\eta_{a}^{\mathbf{i}} \in \lim \left(\mathcal{T}_{1}^{\mathfrak{m}[A]}\right), \eta_{b}^{\mathbf{i}} \in \lim \left(\mathcal{T}_{1}^{\mathfrak{m}[B]}\right)$ such that:

(1) $\eta_{a}^{\mathbf{i}}\left\lceil\mathbf{i}=\eta_{b}^{\mathbf{i}}\lceil\mathbf{i}\right.$

(2) $\left(\eta_{a}^{\mathbf{i}}\left\lceil s, \rho_{c, t}\lceil s) \in \mathcal{R}^{\mathfrak{m}[A]}\right.\right.$ for each $c \in C_{t}^{*}$

(3) $\left(\eta_{b}^{\mathbf{i}}\left\lceil s, \rho_{c, t}\lceil s) \in \mathcal{R}^{\mathfrak{m}[B]}\right.\right.$ for each $c \in C_{t}^{*}$

and let us prove that we can define $\eta_{a}^{\mathbf{i}+1}, \eta_{b}^{\mathbf{i}+1}$ so that $\eta_{a}^{\mathbf{i}} \uparrow \mathbf{i} \unlhd \eta_{a}^{\mathbf{i}+1}, \eta_{b}^{\mathbf{i}}\left\lceil\mathbf{i} \unlhd \eta_{b}^{\mathbf{i}+1}\right.$, and properties (1),(2),(3) hold with $\mathbf{i}+1$ in place of $\mathbf{i}$. (By continuing this process one therefore eventually obtains two equivalent sequences.)

Write $\eta_{a}=\eta_{a, 0} \frown\left\langle i_{a}\right\rangle^{\frown} \eta_{a, \infty}$, and $\eta_{b}=\eta_{b, 0} \frown\left\langle i_{b}\right\rangle^{\urcorner} \eta_{b, \infty}$, where $\operatorname{lgn}\left(\eta_{a, 0}\right)=\operatorname{lgn}\left(\eta_{b, 0}\right)=$ i. By definition, $\eta_{a, 0}=\eta_{b, 0}$ and for every $c \in C_{t}^{*}$,

$$
\left(\eta_{a, 0}, \rho_{c, t}\right) \in \mathcal{R}^{\mathfrak{m}[A]} \cap \mathcal{R}^{\mathfrak{m}[B]} .
$$

There are three cases.

(Case 1) $\mathbf{i} \notin B$. In this case, $\mathbf{i}$ is not an active level for $B$, so we define $\eta_{a}^{\mathbf{i}+1}=\eta_{a}^{\mathbf{i}}$, and define $\eta_{b}^{\mathbf{i}+1}=\eta_{b, 0} \frown\left\langle i_{a}\right\rangle^{\frown} \eta_{b, \infty}$, i.e. replace $i_{b}$ by $i_{a}$. [Since we defined $\mathfrak{m}[A], \mathfrak{m}[B]$ using the same background sequence of graphs, it doesn't matter whether $\mathbf{i} \in A$ or not, recalling Remark 12.4. So applying Claim 12.5, we conclude that $\left(\eta_{b}^{\mathbf{i}+1}\left\lceil s, \rho_{c, t}\lceil s) \in \mathcal{R}^{\mathfrak{m}[B]}\right.\right.$ for each $c \in C_{t}^{*}$.

(Case 2) $\mathbf{i} \notin A$. In this case, since $\mathbf{i}$ is not an active level for $A$, define $\eta_{b}^{\mathbf{i}+1}=\eta_{b}$, and define $\eta_{a}^{\mathbf{i}+1}=\eta_{a, 0} \wedge\left\langle i_{b}\right\rangle^{\frown} \eta_{a, \infty}$, i.e. replace $i_{a}$ by $i_{b}$, and again use Remark 12.4 (if necessary) and Claim 12.5 .

(Case 3) $\mathbf{i} \in A \cap B$. If $i_{a}=i_{b}$, define $\eta_{a}^{\mathbf{i}+1}=\eta_{a}^{\mathbf{i}}$ and $\eta_{b}^{\mathbf{i}+1}=\eta_{b}^{\mathbf{i}}$. If $i_{a} \neq i_{b}$, then since we defined $\mathfrak{m}[A], \mathfrak{m}[B]$ using the same background sequence of graphs, by Claim 12.5 we may without loss of generality use $i_{a}$ : that is, define $\eta_{a}^{\mathbf{i}+1}=\eta_{a}$, and define $\eta_{b}^{\mathbf{i}+1}=\eta_{b, 0} \bumpeq\left\langle i_{a}\right\rangle^{\frown} \eta_{b, \infty}$.

In this way we eventually construct two equal sequences, so $\eta_{*}$ is well defined, so (d) is satisfied, and as this was sufficient to prove (C), we are done.

${ }^{33}$ notice that $\eta_{a}, \eta_{b}$ satisfy these conditions for $\mathbf{i}=0$ 


\section{FURTher DISCUSSION AND OPEN QUESTIONS}

In the late sixties when Keisler's order was defined, it was natural to conjecture that it had a small finite number of classes (see the introduction to [26]). Though it was quickly understood that the order might give an interesting calibration of theories (see 9] and also [31]), it long remained reasonable to believe that the order's power to give model theoretic information would be tied to its simplicity. We are now at a surprising mathematical juncture, where the order has become very complicated, but without losing its tight connection to and calibration of modeltheoretic structure. To communicate some of our excitement, we include a broad list of questions.

A. Saturated models of simple theories. Determining Keisler's order on the stable theories required developing the stability theory to prove a characterization of the saturated models of stable theories (see [33. Theorem III.3.10 and 25] Question 10.4): essentially, that for a model of a complete countable stable theory to be $\lambda^{+}$saturated it suffices that it is $\aleph_{1}$-saturated and that every maximal indiscernible set is large. [The theorem is stronger: $\aleph_{1}$-saturated is really $\kappa(T)$-saturated and the theory need not be countable; for us, regular ultrapowers of models of countable theories are $\aleph_{1}$-saturated, so this statement suffices.] What, in simple unstable theories, are the right analogues of maximal indiscernible sets?

Problem 13.1. In light of the results of this paper, formulate a plausible conjecture of a characterization of $\lambda^{+}$-saturated models of simple theories.

B. Variants of the construction.

Discussion 13.2. Our main construction fixes $\bar{m}, \bar{E}$, and $\Xi$; varying these inputs one would have different theories.

Discussion 13.3. We have written the present construction for a single fast sequence $\bar{m}$ and a family of independent level functions. This was a decision to make the structure of the ideal clearer, among other things. But we might also have written the construction, without level functions, simply for continuum many sequences growing at very different rates (which is, in some sense, what the level functions were formally coding). Looking from this second point of view may give a different perspective on how growth rates of finite families affect model theoretic structure.

C. Interactions with forking.

Question 13.4. For every low simple $T$, is there a "very simple" $T$ equivalent to it, for example, a theory which is simple rank one?

Question 13.5. Can the continuum many incomparable classes be reproduced, in ZFC, within the simple non low theories? Within the non simple theories?

Discussion 13.6. For 13.5, it may be reasonable to consider set theoretic hypotheses such as a measurable or supercompact cardinal, recalling [25]. We may also ask the parallel question for $\unlhd^{*}$.

For computability theorists, a natural question may be:

Question 13.7. Is the structure of the Turing degrees embeddable into Keisler's order? 
E. Questions about ultrafilter construction. An important part of the argument above is constructing ultrafilters, and it may be fruitful to further investigate methods from iterated forcing.

Discussion 13.8. Recall that one way of measuring "size" of a regularizing family $\left\{X_{\alpha}: \alpha<\lambda\right\}$ in an ultrafilter $\mathcal{D}$ on $\lambda$ is to look at the sequence of integers $\left\{n_{t}: t \in \lambda\right\}$ where $n_{t}=\left\{\alpha<\lambda: t \in X_{\alpha}\right\}$. Say that a regularizing family is below a nonstandard integer if its size is. Flexible ultrafilters are those having a regularizing family below any nonstandard integer [17. Each of the ultrafilters we build here, by virtue of its connection to certain integer sequences, has a certain amount of flexibility appropriate to those sequences. There remain very interesting open questions about the extent to which flexibility (which is equivalent to "OK") may be separated from goodness, such as Dow's 1985 question, for references and recent work see e.g. Problems 3.5 and 3.6 of [27. It may be interesting to investigate whether the new family of filters built here, of apparently intermediate flexibility, sheds light on the landscape around these questions, as our methods suggest further ways of engineering the relation of "sizes" of filters and of sequences.

$F$. The minimal simple class, and the maximal class. Recall that the theory $T_{\mathbf{r g}}$ of the random graph is minimum among the unstable theories in Keisler's order. There is a set-theoretic characterization of its class (i.e., there is a necessary and sufficient condition for regular ultrafilters to be good for $T_{\mathrm{rg}}$ ), but to date there is no model-theoretic characterization, indeed no model theoretic characterization of any unstable equivalence class. A natural place to begin is:

Problem 13.9. Give a model theoretic characterization of the class of the theory of the random graph in Keisler's order.

Any reasonable list of open problems on Keisler's order should recall the parallel of 13.9, one of the major questions on the table. (See [21.)

Problem 13.10. Give a model theoretic characterization of the maximal class in Keisler's order, and under $\unlhd^{*}$ without instances of GCH.

G. Variants of Keisler's order. It is a very interesting and natural question to consider what less fine variants of Keisler's order may show about the structure of simple theories, and whether such variants may be found whose number of equivalence classes is finite. We plan to say more about this in future papers.

H. Building blocks of simple theories.

Discussion 13.11. These theories we have built appear quite different from the theories witnessing the infinite descending chain in Keisler's order, which were sums of certain generic $n$-free $k$-hypergraphs, studied originally by Hrushovski [7] (for [26], we used the case $n=k+1$ ). Such theories may be thought of as encoding "pure amalgamation problems."

Indeed, we originally built the precursor to the present theories in 28, to witness Keisler-incomparability with the $T_{k+1, k}$ 's. The role of the new theory in [28, this precursor of the $T_{\mathfrak{m}}$ 's, was in some sense to replace a certain canonical non-low theory in the known, non-ZFC incomparability arguments [37, [22]. We might describe these theories as containing enough of a finite approximation to forking to retain incomparability, but without actually forking. We verified in 2.13 above that 
the theory of 28] fits in the present framework, though the background Boolean algebras in the two papers are quite different. What does this picture tell us about the building blocks of complexity in simple theories? What interesting non-trivial interactions may occur within simplicity between the weak avatars of forking (the uniform inconsistency along various finite quotient sets) in the $T_{\mathfrak{m}}$ 's, and the inconsistency arising from amalgamation in the $T_{n, k}$ 's?

I. Hypergraph regularity. Not unrelated to Problem 13.1, the above discussion of graphs and hypergraphs suggests the following speculative question. The theories we have built in the present paper are really fundamentally graphs (layered across predicates). The key relation is a binary relation, and the key underlying densities are densities of bipartite graphs. Is uniform incomparability across a family necessarily a graph (binary) phenomenon? Recalling that the hypergraph analogues of Szemerédi phenomena are known [6], [32, 35] we may ask 34

Question 13.12. Is there a true "hypergraph analogue" of our construction? For instance, can one construct a family of simple theories whose only forking comes from equality, by analogy to what we have done here, which reflect in some fundamental way the densities of certain families of finite 3-uniform hypergraphs, and which themselves form a higher layer of uniform incomparability phenomena in Keisler's order which is not explained by their restrictions to graphs?

Understanding in either direction may significantly change our understanding of dividing lines in simple theories.

\section{REFERENCES}

[1] B. Bollobás, Random Graphs, second edition. Cambridge University Press, 2001.

[2] C.C. Chang and H. J. Keisler, Model Theory, third edition, 1990.

[3] W. W. Comfort and S. Negrepontis, The Theory of Ultrafilters, Springer-Verlag, Berlin, 1974.

[4] M. Džamonja and S. Shelah, "On $\triangleleft^{*}$-maximality," Ann. Pure Appl. Logic 125 (2004) 119158. Extended edition available at http://shelah.logic.at/files/692.pdf

[5] R. Engelking and M. Karłowicz, "Some theorems of set theory and their topological consequences." Fund. Math. 57 (1965) 275-285.

[6] W. T. Gowers. "Hypergraph regularity and the multidimensional Szemerédi theorem." Ann. of Math. (2) 166 (2007), no. 3, 897-946.

[7] E. Hrushovski, "Pseudo-finite fields and related structures" in Model theory and applications (ed. L. Bélair et al), pp. 151-212, Quad. Math. 11 (Seconda Universita di Napoli, 2002).

[8] T. Jech, Set Theory. Third edition. Springer-Verlag, New York.

[9] H. J. Keisler, "Ultraproducts which are not saturated." J. Symbolic Logic 32 (1967) 23-46.

[10] H. J. Keisler, "The Ultraproduct Construction." In Ultrafilters Across Mathematics ed. by V. Bergelson et. al., Contemporary Mathematics 530 (2010), pp. 163-179, Amer. Math. Soc. see also https://www.math.wisc.edu/ keisler/ultraproducts-web-final.pdf .

[11] J. Komlós and M. Simonovits, "Szemerédi's Regularity Lemma and its applications in graph theory." (1996) In Combinatorics: Paul Erdös is Eighty, Vol. 2 (D. Miklós, V. T. Sós and T. Szönyi, eds), Bolyai Society Math. Studies, Keszthely, Hungary, pp. 295-352.

[12] K. Kunen, Set Theory, revised edition, College Publications, 2013.

[13] M. Malliaris. Ph.D. thesis, University of California, Berkeley, 2009.

[14] M. Malliaris. "Realization of $\varphi$-types and Keisler's order." Ann Pure Appl Logic 157 (2009) $220-224$.

[15] M. Malliaris. "The characteristic sequence of a first-order formula." J Symb Logic 75, 4 (2010) $1415-1440$.

\footnotetext{
${ }^{34}$ Added in revision: Part of this question, constructing theories which are hypergraph analogues of the theories $T_{\mathfrak{m}}$, is now addressed in 30.
} 
[16] M. Malliaris, "Hypergraph sequences as a tool for saturation of ultrapowers." J Symb Logic 77, 1 (2012) 195-223.

[17] M. Malliaris. "Independence, order, and the interaction of ultrafilters and theories." Ann Pure Appl Logic 163 (2012) 1580-1595.

[18] M. Malliaris. "Model theory and ultraproducts." Proceedings of the 2018 ICM. arXiv:1803.07118

[19] M. Malliaris and S. Shelah. "Regularity lemmas for stable graphs." Trans. Amer. Math Soc, 366 (2014), 1551-1585.

[20] M. Malliaris and S. Shelah. "A dividing line within simple unstable theories." Advances in Math 249 (2013) 250-288.

[21] M. Malliaris and S. Shelah. "Cofinality spectrum theorems in model theory, set theory and general topology." J. Amer. Math. Soc. 29 (2016), 237-297.

[22] M. Malliaris and S. Shelah, manuscript 1530, 2015. See also the appendix to "A new look at interpretability and saturation," Ann. Pure Appl. Logic 170 (2019), no. 5, 642-671.

[23] M. Malliaris and S. Shelah. "Saturating the random graph with a family of small range." In Logic Without Borders, eds. Hirvonen, Kontinen, Kossak, Villaveces. DeGruyter, 2015.

[24] M. Malliaris and S. Shelah. "A new look at interpretability and saturation." Ann Pure Appl Logic 170, 5 (2019) 642-671.

[25] M. Malliaris and S. Shelah. "Existence of optimal ultrafilters and the fundamental complexity of simple theories." Advances in Math. 290 (2016) 614-681.

[26] M. Malliaris and S. Shelah, "Keisler's order has infinitely many classes." Israel J. Math. 224 (2018), no. 1, 189-230.

[27] M. Malliaris and S. Shelah, "Open problems on ultrafilters and some connections to the continuum." Contemp. Math. vol. 690 (2017) pps. 145-160. Caicedo, Cummings, Koellner, Larson, eds.

[28] M. Malliaris and S. Shelah. "An example of a new simple theory." Contemp. Math. vol. 752 (2020) pps. 121-152. Coskey and Sargsyan, eds.

[29] M. Malliaris and S. Shelah, "A separation theorem for simple theories." Accepted, Trans. Amer. Math Soc. arXiv:1810.09604

[30] M. Malliaris and S. Shelah, "New simple theories from hypergraph sequences." Manuscript 1206, 14 pages.

[31] M. Morley, mathscinet review MR0218224 of 9].

[32] V. Rödl, B. Nagle, J. Skokan, M. Schacht, and Y. Kohayakawa. "The hypergraph regularity method and its applications." PNAS 102(23): 8109-8113, 2005.

[33] S. Shelah, Classification Theory and the number of non-isomorphic models, first edition 1978, rev. ed., North-Holland 1990. PDF files available at shelah.logic.at/class/.

[34] S. Shelah and J. Spencer, "Zero-one laws for sparse random graphs." JAMS, 1 (1988) 97-115.

[35] T. Tao, "A variant of the hypergraph removal lemma." J. Combin. Theory Ser. A 113 (2006), $1257-1280$.

[36] C. Terry and J. Wolf, "Stable arithmetic regularity in the finite-field model," Bull. London Math. Soc. 51 (2019), no. 1, 70-88.

[37] D. Ulrich, "Keisler's order is not linear, assuming a supercompact." J. Symb. Log. 83 (2018), no. 2, 634-641.

Department of Mathematics, University of Chicago, 5734 S. University, Chicago, IL 60637, USA

Email address: mem@math.uchicago.edu

Einstein Institute of Mathematics, Edmond J. Safra Campus, Givat Ram, The HeBrew University of Jerusalem, Jerusalem, 91904, Israel, and Department of Mathematics, Hill Center - Busch Campus, Rutgers, The State University of New Jersey, 110 Frelinghuysen Road, Piscataway, NJ 08854-8019 USA

Email address: shelah@math.huji.ac.il

URL: http://shelah.logic.at 